\title{
Cancellous bone and theropod dinosaur locomotion. Part I - An examination of cancellous bone architecture in the hindlimb bones of theropods
}

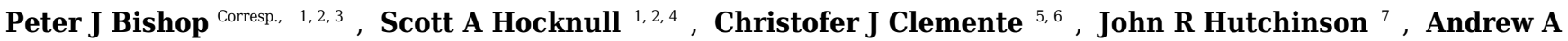 \\ Farke $^{8}$, Belinda R Beck ${ }^{2,9}$, Rod S Barrett ${ }^{2,3}$, David G Lloyd ${ }^{2,3}$ \\ ${ }^{1}$ Geosciences Program, Queensland Museum, Brisbane, Queensland, Australia \\ 2 School of Allied Health Sciences, Griffith University, Gold Coast, Queensland, Australia \\ ${ }^{3}$ Gold Coast Orthopaedic Research, Engineering and Education Alliance, Menzies Health Institute Queensland, Australia \\ 4 School of Biosciences, University of Melbourne, Melbourne, Victoria, Australia \\ 5 School of Science and Engineering, University of the Sunshine Coast, Maroochydore, Queensland, Australia \\ 6 School of Biological Sciences, University of Queensland, Brisbane, Queensland, Australia \\ 7 Structure and Motion Laboratory, Department of Comparative Biomedical Sciences, Royal Veterinary College, Hatfield, Hertfordshire, United Kingdom \\ 8 Raymond M. Alf Museum of Paleontology at The Webb Schools, Claremont, California, United States of America \\ 9 Exercise and Human Performance, Menzies Health Institute Queensland, Australia \\ Corresponding Author: Peter J Bishop \\ Email address: peter.bishop@qm.qld.gov.au
}

This paper is the first of a three-part series that investigates the architecture of cancellous ('spongy') bone in the main hindlimb bones of theropod dinosaurs, and uses cancellous bone architectural patterns to infer locomotor biomechanics in extinct non-avian species. Cancellous bone is widely known to be highly sensitive to its mechanical environment, and has previously been used to infer locomotor biomechanics in extinct tetrapod vertebrates, especially primates. Despite great promise, cancellous bone architecture has remained little utilized for investigating locomotion in many other extinct vertebrate groups, such as dinosaurs. Documentation and quantification of architectural patterns across a whole bone, and across multiple bones, can provide much information on cancellous bone architectural patterns and variation across species. Additionally, this also lends itself to analysis of the musculoskeletal biomechanical factors involved in a direct, mechanistic fashion.

On this premise, computed tomographic and image analysis techniques were used to describe and analyse the three-dimensional architecture of cancellous bone in the main hindlimb bones of theropod dinosaurs for the first time. A comprehensive survey across many extant and extinct species is produced, identifying several patterns of similarity and contrast between groups. For instance, more stemward nonavian theropods (e.g., ceratosaurs and tyrannosaurids) exhibit cancellous bone architectures more comparable to that present in humans, whereas species more closely related to birds (e.g., paravians) exhibit architectural patterns bearing greater similarity to those of extant birds. Many of the observed patterns may be linked to particular aspects of locomotor biomechanics, such as the degree of hip or knee flexion during stance and gait. A further important observation is the abundance of markedly oblique trabeculae in the diaphyses of the femur and tibia of birds, which in large species produces spiralling patterns along the endosteal surface. Not only do these observations provide new insight into theropod anatomy and behaviour, they also provide the foundation for mechanistic testing of locomotor hypotheses via musculoskeletal biomechanical modelling. 


\section{Cancellous bone and theropod dinosaur locomotion.}

\section{Part I - An examination of cancellous bone}

\section{3 architecture in the hindlimb bones of theropods}

4

5

P.J. Bishop ${ }^{1,2,3, *}$, S.A. Hocknull1,2,4, C.J. Clemente ${ }^{5,6}$, J.R. Hutchinson ${ }^{7}$, A.A. Farke ${ }^{8}$, B.R. Beck $^{2,9}$, R.S. Barrett ${ }^{2,3}$ and D.G. Lloyd ${ }^{2,3}$.

${ }^{1}$ Geosciences Program, Queensland Museum, Brisbane, Queensland, Australia.

${ }^{2}$ School of Allied Health Sciences, Griffith University, Gold Coast, Queensland, Australia.

${ }^{3}$ Gold Coast Orthopaedic Research, Engineering and Education Alliance, Menzies Health Institute Queensland.

${ }^{4}$ School of Biosciences, University of Melbourne, Melbourne, Victoria, Australia.

${ }^{5}$ School of Science and Engineering, University of the Sunshine Coast, Maroochydore, Queensland, Australia.

${ }^{6}$ School of Biological Sciences, The University of Queensland, Brisbane, Queensland, Australia.

${ }^{7}$ Structure and Motion Laboratory, Department of Comparative Biomedical Sciences, Royal Veterinary College, Hatfield, Hertfordshire, UK.

${ }^{8}$ Raymond M. Alf Museum of Paleontology at The Webb Schools, Claremont, California, USA. ${ }^{9}$ Exercise and Human Performance, Menzies Health Institute Queensland.

*corresponding author at current address: Structure and Motion Laboratory, Department of Comparative Biomedical Sciences, Royal Veterinary College, Hatfield, Hertfordshire, UK; pbishop@,rvc.ac.uk.

\section{$\underline{\text { I.1 Abstract }}$}


30 This paper is the first of a three-part series that investigates the architecture of cancellous ('spongy') bone in the main hindlimb bones of theropod dinosaurs, and uses cancellous bone architectural patterns to infer locomotor biomechanics in extinct non-avian species. Cancellous bone is widely known to be highly sensitive to its mechanical environment, and has previously been used to infer locomotor biomechanics in extinct tetrapod vertebrates, especially primates. Despite great promise, cancellous bone architecture has remained little utilized for investigating locomotion in many other extinct vertebrate groups, such as dinosaurs. Documentation and quantification of architectural patterns across a whole bone, and across multiple bones, can provide much information on cancellous bone architectural patterns and variation across species. Additionally, this also lends itself to analysis of the musculoskeletal biomechanical factors involved in a direct, mechanistic fashion.

On this premise, computed tomographic and image analysis techniques were used to describe and analyse the three-dimensional architecture of cancellous bone in the main hindlimb bones of theropod dinosaurs for the first time. A comprehensive survey across many extant and extinct species is produced, identifying several patterns of similarity and contrast between groups. For instance, more stemward non-avian theropods (e.g., ceratosaurs and tyrannosaurids) exhibit cancellous bone architectures more comparable to that present in humans, whereas species more closely related to birds (e.g., paravians) exhibit architectural patterns bearing greater similarity to those of extant birds. Many of the observed patterns may be linked to particular aspects of locomotor biomechanics, such as the degree of hip or knee flexion during stance and gait. A further important observation is the abundance of markedly oblique trabeculae in the diaphyses of the femur and tibia of birds, which in large species produces spiralling patterns along the endosteal surface. Not only do these observations provide new insight into theropod anatomy and behaviour, they also provide the foundation for mechanistic testing of locomotor hypotheses via musculoskeletal biomechanical modelling. 
$60 \quad$ I.2 Introduction

61

\section{I.2.1 Background}

63

Perhaps more than any other group of extinct vertebrates (except hominin primates), dinosaurs have been the subject of extensive research into a wide variety of aspects concerning their palaeobiology. One such aspect is their manner of locomotion, which has often been the topic of much debate. Locomotion has played an important role in arguments surrounding dinosaur physiology, behaviour and palaeoecology (Alexander 1989; Bakker 1980; Bakker 1986; Bell \& Snively 2008; Horner \& Lessem 1993; Molnar \& Farlow 1990; Ostrom 1969; Paul 1988; Paul 2008; Pontzer et al. 2009; Thomas \& Farlow 1997; Thulborn 1984). Movement has also been important to understanding dinosaur evolution. For example, approximately three-quarters of the features that distinguish dinosaurs from other animals relate to their erect (parasagittal), ancestrally bipedal posture and locomotion (Brusatte 2012; Novas 1996). Furthermore, much of dinosaur evolution was accompanied by major changes in locomotor morphology, and by inference, behaviour (Carrano 2000; Carrano 2005; Gatesy 2002; Gatesy \& Middleton 1997; Hutchinson \& Allen 2009; Maidment et al. 2014; Middleton \& Gatesy 2000; Novas 1996).

The only primary evidence of dinosaur locomotion available to palaeontologists is the fossils left behind, either body fossils (bones) or trace fossils (footprints and trackways). Fossil footprints and trackways are the most direct line of evidence of locomotion in extinct dinosaurs (Farlow et al. 2012; Gatesy et al. 1999; Gillette \& Lockley 1989; Lockley 1991; Thulborn 1990). However, footprints and trackways do not provide direct insight into the movement or coordination of individual limb segments except for the distal limb; moreover, they cannot be definitively assigned to a particular trackmaker (Hutchinson \& Gatesy 2006; Lockley 1991; Thulborn 1990). In contrast, the associated bones of the animal's skeleton can be positively assigned to a given species, and if preserved well can provide insight into parts of the animal that never touched the substrate.

Owing to their often large size and comparatively detailed body fossil record, many investigations have examined how osteology may relate to locomotor behaviour in extinct 
91 dinosaurs. These studies have typically focused on externally visible features, such as bone

92 shapes or proportions (e.g., Carrano 1998; Carrano 2001; Carrano 2005; Christiansen 1999;

93 Coombs 1978; Gatesy 1991b; Gatesy \& Middleton 1997; Maidment \& Barrett 2014; Maidment et

94 al. 2012), joint range of motion (e.g., Mallison 2010a; Mallison 2010b; Paul 1998) or geometrical

95 relationships between inferred muscle lines of action and joints (e.g., Bates et al. 2012; Carrano

96 2000; Hutchinson et al. 2005; Hutchinson et al. 2008; Maidment et al. 2014; Russell 1972). The

97 insight such studies can provide are usually only general, often having little bearing for

98 understanding the posture or gait of any one species, and moreover carry the caveat of unknowns

99 of soft tissue influences, which may be substantial (Bonnan et al. 2010; Hutchinson \& Gatesy

100 2006; Tsai \& Holliday 2015). Additionally, these studies may only be able to clarify the range of

101 potential locomotor behaviours used by extinct dinosaurs, rather than the reconstruct the

102 behaviours actually used.

103

104

A further line of osteological evidence that has been frequently investigated is the cross-sectional 105 geometry of the mid-shaft of limb bones (e.g., Alexander 1985; Alexander 1989; Alexander 1991; Christiansen 1997; Christiansen 1998; Cubo et al. 2015; Fariña et al. 1997; Farke \& Alicea 2009; Farlow et al. 1995; Heinrich et al. 1993; Lovejoy et al. 2002; Mazzetta et al. 1998; Wilson \& Carrano 1999). The implicit assumption of such enquiry is that the manner in which cortical bone is distributed around a diaphyseal cross-section is related to the magnitude and direction of bending and torsional stresses it experiences (Biewener 1992; Brassey et al. 2013; Wainwright et al. 1976). Therefore, the geometry of a limb bone's cross-section at midshaft may provide insight into whole-bone loading mechanics, and by extension, locomotor behaviour. However, a growing body of experimental evidence indicates that there is no simple correlation between cortical bone morphology and aspects of bone loading, such as bending direction (Bertram \& Biewener 1988; Biewener \& Taylor 1986; Butcher et al. 2008; Demes 2007; Demes et al. 2001; Demes et al. 1998; Lieberman et al. 2004; Main \& Biewener 2004; Pearson \& Lieberman 2004; Thomason 1995; Wallace et al. 2014). Without a strong comparative framework derived from suitable extant species (if they exist) inferences drawn solely from mid-shaft cortical bone morphology should be viewed with caution (see also Farke \& Alicea 2009). 


\section{I.2.2 Cancellous bone in brief}

One aspect of osteology that has remained understudied by dinosaur palaeontologists is the threedimensional (3-D) architecture of cancellous ('spongy') bone, the other main type of bone tissue found in limb bones. Cancellous bone is found throughout the vertebrate skeleton, including in the ends of long bones, vertebrae, throughout short bones (e.g., those of the wrist and ankle) and between the opposing cortices of many flat bones, such as those of the skull (Carter \& Beaupré 2001; Currey 2002; Martin et al. 1998). This work will only consider cancellous bone in the endochondral bones of the appendicular skeleton. Furthermore, it will not consider medullary bone, the loosely packed bone that is periodically formed in birds (Dacke et al. 1993) and at least some dinosaurs (Hübner 2012; Lee \& Werning 2008; Schweitzer et al. 2005), despite its superficial similarity to cancellous bone. Medullary bone is rapidly laid down to act as a calcium reservoir for the production of eggshells before they are laid, and consequently its tissue is not as mechanically competent as that of other, permanent bone tissues: its primary function is metabolic, rather than mechanical (Currey 2002).

The macroscopic architecture of cancellous bone is characterized by a complex, 3-D lattice-like array of interlinking bony struts called trabeculae, from the Latin trabecula, meaning 'small beam' (Fig. 1). The shape of individual trabeculae may be rod-like, plate-like or some variant in between (Singh 1978). Despite being not as mechanically competent as cortical bone, cancellous bone forms a key component of the skeleton; in humans, it comprises some $70 \%$ of the whole skeleton by volume (Huiskes 2000).

The highly complex macrostructure of cancellous bone gives it an exceptionally high ratio of surface area to volume, which makes it a useful reservoir for calcium homeostasis (Clarke 2008; Swartz et al. 1998). More importantly, this high surface area also leads to a rate of remodelling that is an order of magnitude greater than that of cortical bone; in humans, some $25 \%$ by volume

151 is remodelled per year, compared to 2-3\% for cortical bone (Clarke 2008; Huiskes et al. 2000; 
153 changes in its mechanical environment more quickly than cortical bone. There is an every-

154

155

156

157

158

159

160

161

162

163

164

165

166

167

168

169

170

171

172

173

174

growing body of empirical evidence, derived from both experimental and comparative studies, demonstrating how cancellous bone is highly sensitive and well adapted to its mechanical environment. Moreover, when this mechanical environment changes, cancellous bone is able to adapt its architecture in an accurate and predictable fashion. Much of this work has been recently reviewed in detail by Kivell (2016), and will not be discussed further here.

\section{I.2.3 The fabric of cancellous bone (and why cancellous bone shows directionality)}

A salient observation of previous studies is that the orientation of trabeculae (i.e., the fabric of the cancellous bone architecture) is a fundamental component of how cancellous bone is adapted to its environment. Indeed, fabric anisotropy is one of the most important parameters in determining the mechanical behaviour of cancellous bone, second only to bone volume fraction, a measure of porosity (Cowin 1997; Goldstein et al. 1993; Kabel et al. 1999; Maquer et al. 2015; Mittra et al. 2005; Odgaard et al. 1997; Turner 1992; Turner et al. 1990; Ulrich et al. 1999). Furthermore, the principal material directions ${ }^{1}$ in cancellous bone are very closely aligned with the principal fabric directions of its architecture (Fig. 2); that is, the principal axes of the mechanical compliance matrix and fabric tensors are closely aligned (Odgaard et al. 1997; Turner et al. 1990)². Moreover, the degree of fabric anisotropy relates closely with the degree of anisotropy of the mechanical properties: the relative magnitudes of eigenvalues of the fabric tensor closely match that of their respective compliance matrix eigenvalues (Odgaard et al. 1997) . $^{3}$

\footnotetext{
${ }^{1}$ In an anisotropic continuous material, there exist a number of directions in which a given mechanical property (e.g., stiffness) is at its greatest or is lowest magnitude; these are its principal directions. In an orthotropic material such as bone (Cowin 1986; Keaveny et al. 2001; Pidaparti \& Turner 1997), there are two such directions (one maximum, one minimum), which are orthogonal; there is also a third principal direction which is mutually orthogonal to the first two and is a minimax (intermediate). Perfectly isotropic materials have no principal directions, for each mechanical property is the same in every direction.

${ }^{2}$ The compliance matrix $\mathbf{C}$ of a volume of material is a square matrix of order six (generalized Hooke's Law for anisotropic materials) that describes its mechanical properties in terms of values of Young's modulus, shear modulus and Poisson's ratio. The fabric tensor $\mathbf{H}$ is a positive definite second-rank tensor that quantitatively describes the 3-D microstructural arrangement of trabeculae in a volume of cancellous bone (Cowin 1986). The principal axes of $\mathbf{C}$ and $\mathbf{H}$ are given by their eigenvectors (see next footnote).

${ }^{3}$ For a given matrix $\mathbf{A}$, there are one or more vectors $\mathbf{v}$ which maintain their original direction when multiplied by the matrix, although they are dilated by some scaling factor: $\mathbf{A v}=\lambda \mathbf{v}$. These vectors $\mathbf{v}$ are the matrix's eigenvectors,
} 
176 In a comparative context, many previous studies have also demonstrated that differences in

177 cancellous bone fabric direction are indicative of differences in locomotor behaviour, presumably

178 because different behaviours (e.g., joint kinematics) engender different loading regimes and

179 directions thereof (Amson et al. 2017; Barak et al. 2013; Biewener et al. 1996; Carter \& Beaupré

180 2001; Goldstein et al. 1991; Kamibayashi et al. 1995; Matarazzo 2015; Podsiadlo et al. 2008;

181 Radin et al. 1982; Ryan \& Ketcham 2005; van der Meulen et al. 2006). When the loading

182 regimes change, cancellous bone fabric direction also changes, in a highly predictable fashion

183 (Barak et al. 2011; Polk et al. 2008; Pontzer et al. 2006) (Fig. 3). These observations also appear

184 to apply across species as well as within species, as demonstrated by work on several species of

185 primate (Barak et al. 2013; Ryan \& Ketcham 2005).

186

187

A strong correspondence between the directionality of cancellous bone and in vivo mechanical

loading was first suggested more than 150 years ago (von Meyer 1867; Ward 1838). This became widely publicised by Wolff (1892) as the trajectorial theory, which was proposed as an overarching paradigm that related cancellous bone architecture to its mechanical environment. In its modern formulation (Cowin 2001), the trajectorial theory can be stated thus: at remodelling equilibrium (Cowin 1986), the principal material directions of a given volume of cancellous bone are aligned with principal stress trajectories ${ }^{4}$, but only at spatial scales at which the cancellous bone can be treated as a continuous material (Fig. 4). The continuum scale is the scale at which the mechanical behaviour of a volume of cancellous bone structure can be replaced by a set of material properties that are averaged across the same volume. Only at this scale, or larger, can the averaged architecture and mechanical properties of cancellous bone be legitimately compared with the averaged network of principal stress trajectories (Cowin et al. 1992; Martin et al. 1998; Oxnard \& Yang 1981; Tsubota et al. 2002; Tsubota et al. 2009). The spatial scale at which the continuum concept can be invoked for cancellous bone has been suggested to be at least three to five times trabecular spacing (Cowin 2001; Cowin et al. 1992; Harrigan et al. 1988).

and the scaling factors $\lambda$ are the matrix's eigenvalues. The relative magnitudes of the eigenvalues describe the relative extent to which the matrix $\mathbf{A}$ is oriented in each direction given by the eigenvectors.

${ }^{4}$ When a volume of material is under stress due to applied load, there will be three directions in which the shear component is zero; that is, only normal stresses (compressive or tensile) occur in these directions. The normal stresses in these directions are termed the principal stresses, and tangent lines to these directions form a network of principal stress trajectories. These trajectories essentially show how compressive and tensile forces are distributed throughout a body under loading. 
203 The trajectorial theory of cancellous bone architecture has received strong support from many

204

205

206

207

208

209

210

211

212

213

214

215

216

217

218

219

220

221

222

223

224

225

226

227

228

229

230

231

232

experimental (Biewener et al. 1996; Lanyon 1974; Su et al. 1999) and theoretical studies

(Beaupré et al. 1990; Carter et al. 1989; Currey 2002; Gefen \& Seliktar 2004; Giddings et al. 2000; Hayes \& Snyder 1981; Jacobs 2000; Jacobs et al. 1997; Koch 1917; Miller et al. 2002;

Pauwels 1980; Rudman et al. 2006; Sverdlova 2011; Vander Sloten \& Van der Perre 1989), which have repeatedly shown striking similarity between cancellous bone fabric and principal stress trajectories generated from physiological loading. However, whilst it aptly describes the phenomenological association between cancellous bone architecture and its mechanical environment, the trajectorial theory does not link the two together via a mechanistic explanation. Such a mechanistic explanation was provided by Fyhrie \& Carter (1986), who demonstrated that strain energy density (SED) in a given volume of cancellous bone is minimized when the architecture is anisotropic such that (i) the direction of maximum stiffness is parallel to that of the maximum principal stress, (ii) the direction of minimum stiffness is parallel to that of the minimum principal stress, and (iii) the direction of the intermediate stiffness is parallel to that of the intermediate principal stress. Thus, if SED is a stimulus for trabecular remodelling, cancellous bone adaptation at the continuum level can be mechanistically linked to remodelling activites at the cellular level.

More recent computational modelling studies have shown that SED, or a related measure such as strain or stress, is indeed likely an important driver of trabecular remodelling. Common to each is the notion of the 'mechanostat' of bone (Christen et al. 2014; Cresswell et al. 2016; Frost 1987;

Frost 2003; Lambers et al. 2013; Schulte et al. 2013): bone remodels through the addition of bone tissue by osteoblasts to areas experiencing high strain (i.e., overloaded areas) and the removal of bone tissue by osteoclasts from areas experiencing low strain (i.e., underloaded areas) (Fig. 5AD). By this process, at remodelling equilibrium all parts of the cancellous structure bear the same amount of strain, or more correctly, their SED is the same. By using a uniform SED as a remodelling objective, numerous continuum-level finite element computational models have predicted bulk density distributions and fabric patterns that accurately reflect reality (Beaupré et al. 1990; Carter \& Beaupré 2001; Carter et al. 1989; Coelho et al. 2009; Jacobs et al. 1997; Kowalczyk 2010; Turner et al. 1997). More impressively, high-resolution simulations of cellular- 
233 level remodelling produce models that spontaneously 'self-trabeculate' from an initially isotropic

234 configuration (Martin et al. 1998). The result of such simulations is a cancellous structure with

235 trabeculae of realistic proportions, and in those models simulating whole bones, life-like whole-

236 bone architectures (Adachi et al. 2001; Boyle \& Kim 2011; Huiskes et al. 2000; Jang \& Kim

237 2008; Jang \& Kim 2010a; Jang \& Kim 2010b; Mullender \& Huiskes 1995; Phillips 2012; Phillips

238 et al. 2015; Ruimerman et al. 2005; Smith et al. 1997; Tsubota et al. 2002; Tsubota et al. 2009;

239 Wang et al. 2012) (Fig. 5E,F). Moreover, these trabeculae, or more correctly, the fabric

240 directions, are aligned with the continuum-level principal stress trajectories, and when the

241 loading regime changes, the model re-adapts to produce a new cancellous architecture, where the

242 trabeculae are aligned with the new continuum-level principal stress trajectories (Adachi et al.

243 2001; Huiskes et al. 2000; Mullender \& Huiskes 1995; Ruimerman et al. 2005; Wang et al.

244 2012). Hence, the trajectorial theory, which is a global pattern observable on the scale of whole

245 bones, may be considered emergent from the local actions of cells.

246

247 A rigid application of the trajectorial theory to cancellous bone would predict that trabeculae are

248 oriented at right angles to each other. However, this is often not the case; in fact, orthogonal

249 intersections seem to be the exception, rather than the rule (Murray 1936). The reason for this

250 apparent paradox is that most bones experience multiple, often diverse loading regimes. It is this

251 adaptation to multiple different loading regimes, each with different principal stress trajectories,

252 that produces the nonorthogonality observed in the majority of cancellous bone architectures

253 (Ben-Zvi et al. 2017; Heřt 1994; Pidaparti \& Turner 1997; Skedros \& Baucom 2007). Thus, only

254 in bones that tend to experience a single loading regime would an orthogonal 'trajectorial

255 structure' be expected in cancellous bone. One such example is the calcaneum of a number of

256 digitigrade mammals, such as sheep (Lanyon 1974), mule deer (Skedros \& Baucom 2007;

257 Skedros et al. 2004; Skedros et al. 2007), horses (Vander Sloten \& Van der Perre 1989),

258 macropod marsupials (Biewener et al. 1996) and cattle (Fig. 6). These bones are loaded in an

259 extremely consistent manner, by the pull on the distal end from the Achilles tendon and

260 superficial digital flexor tendons. A uniform strain energy distribution within a volume of

261 cancellous bone is hence not usually achieved in any single given loading regime (Jang \& Kim

262 2008; van Rietbergen et al. 2003; van Rietbergen et al. 1999). Rather, it is the time-averaged

263 distribution of SED, resulting from multiple daily loading regimes that a bone experiences, which 
264 is uniform and which drives cancellous bone remodelling. This has been demonstrated by

265 numerous computational simulations of the bone remodelling process. Specifically, no one

266 loading regime will lead to replication of all the observed architectural features in a bone; only

267 when multiple loading regimes are considered can all of a bone's cancellous architecture be

268 accounted for by the trajectorial theory (Beaupré et al. 1990; Boyle \& Kim 2011; Carter \&

269 Beaupré 2001; Carter et al. 1989; Coelho et al. 2009; Jacobs et al. 1997; Jang \& Kim 2008; Jang

270 \& Kim 2010a; Jang \& Kim 2010b; Phillips et al. 2015; Sverdlova 2011; Tsubota et al. 2002;

271 Tsubota et al. 2009; Turner et al. 1997).

272

273

274

275

276

277

278

279

280

281

282

283

284

285

286

287

288

289

290

291

292

293

294

\section{I.2.4 Non-mechanical influences on cancellous bone architecture}

Although the architecture of cancellous bone is clearly influenced by its mechanical environment, it may also be influenced by other factors, such as ontogeny and genetics. Epigenetic influences on cancellous bone mechanobiology may also exist, but exactly what these could be, and how much they interact with genetic influences, remains unknown.

Many studies have demonstrated that the cancellous architecture in a particular region of a bone changes considerably throughout the ontogeny of an individual (Abel \& Macho 2011; Gosman \& Ketcham 2009; Gosman et al. 2011; Nafei et al. 2000a; Nafei et al. 2000b; Raichlen et al. 2015; Ryan \& Krovitz 2006; Tanck et al. 2001; Townsley 1948; Volpato 2008; Wolschrijn \& Weijs 2005). Such changes are necessitated by increases in absolute bone size, and it is therefore unsurprising that the most rapid changes occur early in ontogeny, during the growth of an individual (Gosman \& Ketcham 2009; Gosman et al. 2011; Raichlen et al. 2015; Ryan \& Krovitz 2006; Tanck et al. 2001). The timing of these ontogenetic changes in cancellous bone architecture often reflect the timing of ontogenetic changes in locomotor behaviour, especially the initial commencement of sustained locomotor-induced loading (Gosman \& Ketcham 2009; Gosman et al. 2011; Raichlen et al. 2015; Ryan \& Krovitz 2006; Tanck et al. 2001; Townsley 1948; Volpato 2008; Wolschrijn \& Weijs 2005). Therefore, mechanical factors influence cancellous bone remodelling not only in the adult, but across the entire lifespan of an individual.

Given that the adaptation of cancellous bone to its mechanical environment occurs throughout the life of an individual, an interesting proposition arises if the rate at which bone remodels decreases 
295 through ontogeny (Christiansen et al. 2000; Keaveny \& Yeh 2002; Lieberman et al. 2003;

296 Pearson \& Lieberman 2004). That is, the adaptive response of cancellous bone in the adult may

297 not be as proficient as in earlier stages of life. If this occurs, the architecture observed in the adult

298 may reflect, to some degree, the habitual loading experienced during ontogeny, and not just the

299 current habitual loading environment (Carlson et al. 2006; Petterson et al. 2010; Pontzer et al.

300 2006). This phenomenon of ontogenetic inertia has not been investigated in great detail, but a

301 general consideration may nevertheless be made. The potential for ontogenetic inertia in

302 cancellous bone architecture will depend on at least four variables, namely (i) the absolute rate at

303 which cancellous bone remodels, (ii) the lifespan of the individual, (iii) the absolute increase in

304 bone size through ontogeny, and (iv) the degree to which locomotor-induced loading changes

305 through ontogeny.

306

307 Ontogenetic inertia will be minimal in species that have a high rate of bone remodelling

308 compared to their lifespan. For example, adult humans remodel about $25 \%$ by volume of their

309 cancellous bone per year (Huiskes et al. 2000; Parfitt 1983). Given the lengthy lifespan of

310 humans, this implies that cancellous bone will be turned over many times during the life of an

311 individual, erasing the 'signals' of locomotor-induced loading from earlier stages in life.

312 However, there may be a small, immediate component of ontogenetic inertia. This is because

313 bone (re)modelling can only occur on pre-existing bone surfaces (Carter \& Beaupré 2001; Martin

314 et al. 1998; Mullender \& Huiskes 1995), and hence there may be some lag left over between

315 successive 'bone generations'. Over the lifetime of an individual, however, this will be

316 inconsequential. A great increase in the absolute size of limb bones through ontogeny, as seen in

317 humans, will also result in the complete turning over of cancellous bone many times, reducing the

318 magnitude of ontogenetic inertia. If locomotor behaviour does not change appreciably throughout

319 ontogeny, then ontogenetic changes in locomotor-induced bone loading will be minimal.

320 Consequently, the cancellous bone architecture observed in the adult will reflect the current

321 habitual loading environment, because this environment has remained effectively unaltered for a

322 significant length of time. Such a pattern is also observed in humans, where locomotor behaviour

323 effectively matures by the age of four years (Sutherland 1997), and the cancellous bone

324 architecture in the human proximal femur and tibia is effectively unchanged from about nine

325 years of age onward (Gosman \& Ketcham 2009; Ryan \& Krovitz 2006). Minimal ontogenetic 
326 inertia would also be expected for ostriches, which have a high rate of bone remodelling (Currey

327 2003), a sizeable lifespan (Davies 2002), exhibit great increase in bone size from chick to adult,

328 and which show little ontogenetic change in locomotor behaviour as far as limb posture is

329 concerned (Smith et al. 2010). One further consideration is that the magnitude of ontogenetic

330 inertia may also depend on if the bone has experienced relatively 'novel' mechanical loading

331 conditions in its recent past. In such a situation more rapid remodelling may occur in response to

332 these novel loading conditions (Robling et al. 2006; Robling \& Turner 2009), serving to 'erase'

333 older ontogenetic signal and thereby decreasing the magnitude of ontogenetic inertia.

334

335

336

As regards genetic influences on cancellous bone architecture, these influences probably depend on the scale at which the topic is approached. Many studies have investigated the genetic effects on cancellous bone adaptation to mechanical loading, particularly in different strains of mice, and have shown that genetics can indeed modulate cancellous bone mechanobiology (e.g., Havill et al. 2010; Judex et al. 2004; Wallace et al. 2015; Wallace et al. 2012). However, the aforementioned investigations concern within-species differences, and concern very specific regions of a given bone. They hence do not illustrate how genetics influences the adaptation of entire bones, across the skeleton, and across species that load their bones in different manners.

In terms of the architecture of whole bones, genetic factors strongly influence a bone's initial development. The basic structure of the whole bone derives from the systematic expression of positional information encoded in the genome (Lanyon 1996; Lovejoy et al. 2002). Moreover, recent research has indicated that some aspects of the finer-scale architectural features may also be influenced by genetic factors, in addition to mechanical factors. For example, the gross architecture of cancellous bone (such as density distribution) in the adult human ilium appears quite early on during foetal development, well before the onset of locomotor-induced loading, suggestive of genetic influence (Abel \& Macho 2011; Cunningham \& Black 2009a; Cunningham \& Black 2009b). However, such a phenomenon is not observed in the human proximal femur or tibia (Gosman \& Ketcham 2009; Ryan \& Krovitz 2006). The early appearance of an adult-like gross architecture may alternatively result from in utero muscular contractions, producing mechanical stimulation of the developing bones (Abel \& Macho 2011; Cunningham \& Black 2009a; Cunningham \& Black 2009b; Lanyon 1974). Further insight is provided by a second 
357 example, namely the development of the calcaneum in artiodactyl ungulates. In both sheep

358 (Lanyon 1974) and mule deer (Skedros et al. 2004), the cancellous bone architecture observed in

359 the adult calcaneum occurs in the foetus, paralleling the situation in the human ilium. However,

360 when Lanyon and Goodship (reported by Skerry 2000) transected the Achilles tendon of a

361 developing foetal lamb in utero, they found that subsequent prenatal growth resulted in a

362 disorganized architecture in the experimental calcaneum, compared to the contralateral control.

363 This suggests that in some situations at least, prenatal loading can be responsible for the

364 cancellous bone architecture observed in a newborn animal, possibly diminishing the significance

365 of genetic influences.

366

367 In general then, it appears that the influences on a bone's cancellous architecture shift during 368 ontogeny, from a dominant role of genetic influences in early prenatal development (in utero or

369 in ovo), to an increasingly important role for extragenetic stimuli, such as mechanical loading, in

370 later development and postnatal ontogeny (Skedros et al. 2007). That is, a basic, genetically

371 determined template lays out the gross architecture of cancellous bone, which is subsequently

372 built upon and remodelled during postnatal ontogeny in response to mechanical loading.

374 Genetic influences on cancellous bone architecture may also extend across species. If the genetic control of cancellous bone architecture is strong enough, the potential arises that the cancellous architecture observed in a given bone in a particular species may not entirely reflect the loads

377 experienced by the individual in life, but also the loads experienced by the homologous bone 378 somewhere in the past of the species' evolutionary history. For example, if a species of primate that engages in quadrupedal locomotion recently evolved from a species which engaged in leaping locomotion, it may inherit some architectural characteristics from its ancestors. That is, whilst it is a quadruped, its cancellous bone architecture may be somewhat 'leaper-like' in nature (Ryan \& Ketcham 2005). This phenomenon of phylogenetic inertia (Blomberg \& Garland 2002) has received only limited attention in the context of cancellous bone architecture, but what research has been conducted shows only a weak effect, if any (Ryan \& Shaw 2012; Ryan \& Shaw 2013; Scherf et al. 2013; Tsegai et al. 2013). This possibly weak effect further suggests that the architecture of cancellous bone observed in the adult is largely, if not entirely, influenced by mechanical stimuli (Skedros et al. 2007). 
389 The possibility of phylogenetic inertia can only exist if the genome itself (in terms of the allele

390

391

392

393

394

395

396

397

398

399

400

401

402

403

404

405

406

407

408

409

410

411

412

413

414

415

416

417

418

frequencies at the population level) is subject to the influences of the mechanical loading environment a bone experiences in life. That is, the genome that codes for the initial template of cancellous bone architecture is influenced by how the bone is loaded in life. Or, put another way, patterns of bone loading resulting from a particular locomotor behaviour lead to selection on the phenotype, which in turn influences populational allele frequencies over generations, affecting the predetermination of gross architectural features prior to eventual bone use and loading in life (Lanyon 1974; Ryan \& Shaw 2012; Ryan \& Shaw 2013; Saparin et al. 2011; Townsley 1948). If epigenetic factors are involved, then the adaptation through the genome may possibly be achieved very quickly. It would indeed be advantageous to have some form of a blueprint in place for the gross architecture of cancellous bone, because this starts the bone 'off on the right foot' as soon as the animal is born (or hatches). In precocial species, the young have to start locomoting - and often have to keep up with the adults - as soon as they are born (or hatch); with the cancellous bone architecture already somewhat pre-adapted, this would make the bone more structurally efficient from the very first day of postnatal loading (Gorissen et al. 2016).

In light of the above considerations, there appear to be at least three pathways that the relationship between cancellous bone architecture and its mechanical environment can take (Fig.

7):

i. A direct pathway, whereby locomotor-induced bone loading leads to changes in cancellous bone architecture, via adaptive remodelling throughout the lifetime of an individual.

ii. An indirect pathway, whereby patterns of locomotor behaviour lead to selection on the genome (i.e., adaptation over generations), which in turn affects the genetic predetermination of gross architectural features prior to loading.

iii. A direct pathway, whereby prenatal muscular contractions produce bone loading, leading to adaptive remodelling prior to the commencement of postnatal, locomotor-induced loading.

If epigenetic factors are involved in cancellous bone mechanobiology, then a fourth, indirect, pathway would exist. These pathways are not mutually exclusive of each other, and it is likely that they all contribute to the final architecture observed in cancellous bone, to varying degrees. Pathway (i) can explain changes in cancellous bone architecture in response to changes in loading 
419

420

421

422

423

424

425

426

427

428

429

430

431

432

433

434

435

436

437

438

439

440

441

442

443

444

445

446

447

448

449

conditions within individuals, either naturally or experimentally. Pathways (i) and (ii) can explain how cancellous bone architecture reflects the loads experienced in post-natal life, and hence why different cancellous bone architectures reflect different locomotor behaviours, such as those between different species. Pathways (ii) and (iii) can explain the presence of gross architectural features that are characteristic and reflective of adult locomotion, yet which are present in neonates before the onset of locomotor-induced loading.

\section{I.2.5 The utility of cancellous bone in understanding locomotion in extinct, non-avian dinosaurs}

Cancellous bone architecture clearly has great potential utility for better understanding locomotor biomechanics in extinct tetrapods. Most previous studies that have used cancellous bone to test hypotheses of behaviour have focused on extinct primates (Barak et al. 2013; D'Anastasio et al. 2013; DeSilva \& Devlin 2012; Hébert et al. 2012; Macchiarelli et al. 1999; Rook et al. 1999; Ryan \& Ketcham 2002a; Scherf 2008; Skinner et al. 2015; Su \& Carlson 2017; Su et al. 2013), with few studies directed towards other tetrapod groups (Bishop et al. 2015; Moreno et al. 2007; Sues 1978; Thomason 1985). Yet, extinct, non-avian dinosaurs are a group that would be quite suitable for this kind of investigation. Non-avian dinosaurs lived for a very long period of time and in a wide variety of environments, exhibited a diverse array of locomotor morphologies, and their fossils are relatively abundant and often well-preserved. They are also inferred to have had high rates of bone growth and remodelling, comparable to that of extant mammals and birds (Brusatte 2012; Currey 2002; Reid 2012), in contrast to that observed in most extant, sprawling reptiles (Currey 2002; de Ricqlès 1976; Enlow 1969). Especially in the larger species, they also had both lengthy lifespans and a large change in absolute bone size through ontogeny, having hatched from eggs less than $30 \mathrm{~cm}$ in diameter (Horner 2000). They would hence be expected to show minimal ontogenetic inertia in adults.

To the authors' knowledge, only two studies have examined cancellous bone architecture as it relates to aspects of non-avian dinosaur biomechanics, although technological limitations may be 
450 in part responsible for this. In the first (Sues 1978), 'trabeculae' were observed in the enlarged

451 skull domes of pachycephalosaurs, appearing to be oriented appropriately for receiving the forces

452 that might be experienced during head-butting behaviour. However, these 'trabeculae' were later

453 interpreted to be an ontogenetic transitory structure in the growth of the skull bones (Goodwin

454 and Horner, 2004). More recently, cancellous bone in the pedal phalanges of various dinosaur

455 species was imaged using clinical-grade X-ray computed tomographic scanning, and basic

456 phenomenological interpretations were made (Moreno et al. 2007). Similarly, little investigation

457 has been undertaken in the way of cancellous bone architecture in extant birds. In fact, aside from

458 being qualitatively illustrated on several occasions (e.g., Cracraft 1971; Owen 1866; Thompson

459 1942; Townsley 1948), and experimentally manipulated according to different loading patterns

460 (Pontzer et al. 2006), the three-dimensional macrostructure of cancellous bone in the limb bones

461 of birds is virtually unstudied.

462

463 A phenomenological approach has been the dominant theme of most previous studies of 464 cancellous bone architecture in extinct vertebrates. Often, investigation has largely been limited 465 to comparing the architecture of cancellous bone in a given extinct species to that of extant 466 related species (e.g., DeSilva \& Devlin 2012; Macchiarelli et al. 1999; Ryan \& Ketcham 2002a; 467 Scherf 2008; Skinner et al. 2015; Thomason 1985), essentially asking the question "what extant 468 species is the extinct one closest to?" That is, similarity (or difference) in cancellous bone 469 architecture implies similarity (or difference) in locomotor behaviour. Even then, comparisons 470 are often limited to discrete sub-regions of the bone of interest, rather than architectural patterns 471 throughout a whole bone. Whilst this approach may serve as a good starting point, it cannot by 472 itself provide insight into questions of whole-bone loading or musculoskeletal mechanics.

473 Furthermore, whilst such an approach may be useful when applied to a group of animals with 474 close extant relatives of similar morphologies (such as primates), it may not be useful when 475 investigating extinct animals that are quite different from any extant animal group (such as non476 avian dinosaurs).

477

A more appropriate way of investigating cancellous bone architecture in any extinct species is 479 through a holistic, whole-bone, biomechanically-informed approach. By considering the 480 architecture of cancellous bone throughout an entire bone, more insight may potentially be 
481 gained, compared to focusing on a limited number of specific regions (Georgiou et al. 2018;

482 Gross et al. 2014; Kivell 2016; Ryan \& Test 2007; Saparin et al. 2011; Scherf 2008; Skinner et al. 483 2015; Stephens et al. 2016; Su et al. 2013; Tsegai et al. 2013; Tsegai et al. 2017). Even more 484 insight may be possible by considering the architectural patterns of multiple bones, rather than 485 just one (Saers et al. 2016; Stephens et al. 2016; Tsegai et al. 2017). Most of the aforementioned 486 whole-bone studies have focused on how scalar variables (e.g., bone volume fraction) vary 487 throughout a bone. However, as the orientation of cancellous bone fabric is quite telling of 488 loading conditions, and strongly reflects the mechanical performance of cancellous bone tissue as 489 a whole, it is also deserving of considerable research attention. Importantly, cancellous bone 490 fabric can also be related to bone loading mechanics across the scale of whole bones, via the 491 trajectorial theory: principal material directions, and hence principal fabric directions, are aligned 492 493 494 495 496 497 498 with continuum-level principal stresses engendered by physiological loading. Thus, when a whole-bone approach is taken, cancellous bone fabric can be linked with whole-limb musculoskeletal biomechanics in a mechanistic fashion, rather than just phenomenologically.

\section{I.2.6 Outline of this study}

In the present study, and the two subsequent parts of this series (Bishop et al. in review-a; Bishop et al. in review-b), cancellous bone architecture was investigated in one particular sub-group of dinosaurs, the theropods, to demonstrate how the investigation of microstructural characteristics across whole bones has the potential to provide unparalleled insight into questions of posture and loading mechanics. Theropoda include some of the most iconic of extinct animals, as well as the most species-rich group of modern-day terrestrial vertebrates, the birds (Bennett \& Owens 2002; Chiappe \& Witmer 2002; Gauthier 1986; Holtz 2012; Naish 2012; Sereno 1999; Weishampel et al. 2004). Over their 230 million year history, theropods have spanned an incredible range of body size, from the two gram Mellisuga helenae (bee hummingbird) to the eight tonne Tyrannosaurus rex (Dunning 2007; Henderson 1999; Hutchinson et al. 2011), and, despite being exclusively bipedal, have displayed a wide range of locomotor morphologies (Baumel \& Witmer 1993; Carrano 1998; Gatesy \& Middleton 1997; Middleton \& Gatesy 2000; Paul 1988). This 
512 terrestrial locomotion, as well as the consequences of large body size on locomotor performance.

513 Additionally, studies of theropod locomotion are critical to charting the evolution of locomotor

514 behaviour on the line to modern birds, including the origin of a novel locomotor pattern, avian

515 flight (Allen et al. 2009; Gatesy 2002; Heers \& Dial 2012; Hutchinson \& Gatesy 2000).

516

517 Terrestrial locomotion in theropods has received considerable attention over the past three

518 decades, and a substantially more detailed picture of non-avian theropod stance and gait, and its

519 evolution, has emerged. It is now well established that on the line to modern birds, many

520 profound anatomical changes occurred in theropods, including significant modifications of pelvic

521 and hindlimb osteology (Carrano 2000; Gatesy \& Middleton 1997; Hutchinson 2001a;

522 Hutchinson 2001b), musculature and proportions (Carrano \& Hutchinson 2002; Hutchinson

523 2001a; Hutchinson 2001b; Hutchinson 2002), changes to tail length and construction (Gatesy

524 1990; Gatesy 1995; Gatesy 2002; Pittman et al. 2013), and changes in the position of the whole-

525 body centre of mass (Allen et al. 2013). These are inferred to have influenced limb posture, from

526 more upright (less flexed hips and knees) in most forms to more crouched (more flexed hips and

527 knees) in the more derived forms (Bates et al. 2012; Carrano 1998; Gatesy 1990; Gatesy 1991b;

528 Gatesy 1995; Gatesy et al. 2009; Grossi et al. 2014; Hutchinson et al. 2005), as well as the

529 muscular strategies of limb support and propulsion (Gatesy 1990; Gatesy 1995; Gatesy 2002;

530 Hutchinson \& Gatesy 2000). In turn, bone loading mechanics is also inferred to have changed

531 markedly through theropod evolution (Carrano 1998; Farke \& Alicea 2009). All of these changes

532 were set against a backdrop of substantial body size evolution, with sustained miniaturisation

533 occuring along much of the stem lineage (Benson et al. in press; Lee et al. 2014; Turner et al.

534 2007), but also with many instances of secondary gigantism (Benson et al. in press; Carrano

535 2006; Lee et al. 2014). Studies of cancellous bone architecture have the potential to provide new

536 and improved insight on many of these changes.

537

538 Here, the three-dimensional (3-D) architecture of cancellous bone was investigated in the

539 principal hindlimb bones of a variety of extinct, non-avian theropod and extant ground-dwelling

540 bird species. Investigation focused mainly on the direction of the cancellous bone fabric and how

541 this varies spatially throughout a given bone. The reasoning for this is threefold: 
542 1. The direction of fabric alignment is one of the more telling aspects of cancellous bone

543 architecture in terms of identifying differences in locomotor behaviour and bone loading (e.g.,

544 Barak et al. 2011; Barak et al. 2013; Goldstein et al. 1991; Polk et al. 2008; Pontzer et al.

545 2006; Ryan \& Ketcham 2005).

546 2. When considered across the whole bone, the 3-D pattern of fabric directions can be analysed

547 within the framework of the trajectorial theory. This encompassing approach provides greater

548 power to an analysis, because this facilitates direct, mechanistic comparisons of cancellous

549 bone architecture to whole-bone loading, as will be done in Parts II (Bishop et al. in review-a)

550 and III (Bishop et al. in review-b).

551 3. Fabric direction is probably more reliably assessed for fossil specimens, as opposed to other

552 features such as bone volume fraction, trabecular thickness or trabecular spacing. Although

553 these other architectural features can also be useful for interpreting locomotor biomechanics

554 (Kivell 2016), their investigation requires excellent preservation of the entire fossil and very

555 high resolution imaging, the latter of which is difficult (if not impossible) for large bones. So

556 long as the gross structure is preserved and able to be imaged, fabric direction can be assessed.

557 The observations made for theropod limb bones were also compared to those for theropod

558 outgroups (crocodilians and lizards), as well as the other extant obligate biped, humans, which

559 have been very well characterised with respect to cancellous bone architecture and locomotor

560 biomechanics.

561

562 The research presented here in Part I includes first and foremost a comprehensive assessment of

563 the gross architectural patterns in the hindlimb bones of many different theropod species, which

564 constitutes a completely novel dataset. In addition to laying the foundations for future studies, it

565 will facilitate the identification of major patterns of similarity and difference between species and

566 between groups. This in turn can elucidate how cancellous bone architecture may have evolved in

567 theropods, and provide new and unique insight into theropod locomotor biomechanics. The

568 manner of loading that is associated with cancellous bone architecture, and how this may reflect

569 differences in posture, muscle control or gross loading regimes (e.g., bending- versus torsion-

570 dominant), will form the subject of Parts II (Bishop et al. in review-a) and III (Bishop et al. in

571 review-b).

572 


\section{$574 \quad$ I.3 Materials and Methods}

575

576 The sampling and methods used in the present study are outlined in full below. In brief, this study

577 acquired X-ray computed tomographic (CT) scans of the main bones of the hindlimb of a range

578 of avian and non-avian theropods, as well as extant reptilian outgroup species and humans, and

579 used a variety of image processing and analysis techniques to help characterise the architecture of

580 cancellous bone in these elements. Approximately $1.45 \mathrm{~TB}$ of CT scan data was obtained for over

581160 bones, representing at least 44 species (Table 1). Owing to various logistical constraints,

582 these bones were scanned using a variety of machines at a variety of resolutions. Coupled with

583 the fossilization of many of the specimens, these varied resolutions required several different

584 image processing protocols to extract the structural data. Likewise, a number of different

585 analytical approaches were used, some more quantitative than others, to identify the predominant

586 architectural patterns present. For some of the quantitative data, statistical analyses were also

587 conducted to test for correlations of certain architectural features with body size. The whole

588 procedure of data processing and analysis, undertaken on two computers with $\geq 32$ GB of memory

589 and a $2.4 \mathrm{GHz}$ processor each, took approximately six months to complete.

590

591 All scripts and data used are held in the Geosciences Collection of the Queensland Museum, and 592 are available upon request to the Collections Manager. Additionally, a complete copy of the fossil 593 CT scan data is accessioned with the respective museums in which the specimens are housed (see 594 Table 1).

595

596

597

\section{I.3.1 Data acquisition}

598

599 This study focused on the main bones of the hindlimb, the femur, tibia (tibiotarsus ${ }^{5}$ ) and fibula, in 600 a variety of extant avian and non-avian theropods, as well as extant reptilian species, crocodilians

\footnotetext{
5 The terms tibia and tibiotarsus are used in a specific fashion throughout this study. 'Tibia' refers to the bony element per se, whereas 'tibiotarsus' refers to the functional unit of the tibia and proximal tarsals (astragalus and calcaneum). Thus, 'tibiotarsus' is only meaningful when used in reference to theropods and other dinosaurs, in which the three comprising bones are tightly integrated, and in adult birds they become fused. In the other groups of
} 
601 and lizards (Table 1; institutional abbreviations for museum specimens are also detailed here). In 602 addition, data were collected for a representative human specimen, to provide further

603 comparative context; this was an adult male specimen used for teaching purposes, which showed 604 no apparent pathologies. A schematic illustration of the higher-level phylogenetic relationships of 605 the study species is given in Fig. 8.

606

607 For the fossil specimens, careful inspection was undertaken to ensure that the best-preserved and 608 most complete available specimens were studied, avoiding bones, or regions thereof, that had 609 evidence of taphonomic deformation (see also below). Only primarily ground-dwelling species of

610 birds were investigated because, by virtue of spending most or all of their time on the ground,

611 they have well-developed hindlimb locomotor systems. Where possible, only male bird 612 specimens were chosen, so as to preclude the possibility of medullary bone being present and 613 influencing the results (Dacke et al. 1993; but see below). Crocodilians were chosen as they 614 represent the closest extant outgroup of Theropoda. Varanids (monitor lizards) were chosen to 615 represent squamates because most are highly terrestrial species, and their large size better 616 facilitates analysis of cancellous bone architecture compared to smaller species. Generally, only 617 one or two individuals were sampled for a given species. As this study is the first detailed survey 618 of cancellous bone architecture in theropods, and more broadly, saurians, preference was given to 619 maximizing the number of species investigated, rather than achieving a larger number of samples 620 for fewer species, in order to elucidate any general, broad-scale patterns that were present. With 621 sample sizes of usually $n=1$ or 2 , it was hence not possible (or meaningful) to quantify potential 622 intraspecific variation in bone architecture, nor was it possible to examine finer-scale patterns as 623 may occur in association with more subtle differences in anatomy, behaviour or habitat.

625 The 3-D cancellous bone architectural data was acquired through CT scanning of the limb bones.

626 Scanning parameters varied depending on the size and bulk density of the specimen and the 627 locally available scanning machine (details given in Table 1). In all cases, the highest possible 628 resolution and contrast between bone and non-bone phases was sought. Nevertheless, as a 629 consequence of using various scanning modalities on different-sized bones, the resulting scans

animals studied, the presence of a tibiotarsal joint precludes the use of this term. As such, cancellous bone architecture in the proximal tarsals of theropods was also investigated. 
630 had a range of absolute and relative (to bone dimensions) voxel resolutions. This in turn required 631 that multiple techniques for image data processing and analysis were used, as detailed below 632 (sections I.3.2, I.3.3). Regarding the non-avian theropods, more than 40 additional fossil 633 specimens (including of other species) were scanned throughout the course of this study, but 634 owing to various factors (e.g., high density, low contrast between bone and matrix, mineral635 induced scanning artefacts, insufficient scanning power or resolution) their resulting scans were 636 not useful and thus excluded. In addition to the data collected in the present study, data collected 637 by previous studies were also used (Doube et al. 2012; Farke \& Alicea 2009).

638

639

\section{I.3.2 Image data processing}

641

642 The CT scans of each bone were processed using the software Image 1.47

643 (http://imagej.nih.gov/ij/) and Mimics 17.0 (Materialize NV, Belgium), so as to segment the bone 644 from the non-bone phases. All image processing was undertaken by a single person (PJB). Five 645 different image processing protocols were used, depending on differences in the specimens and 646 how they were scanned (Table 1):

647

648

\section{I.3.2.1 Protocol 1 - extant animal micro-CT scans $(\sim 70$ bones $)$}

650

651 Scans were segmented using the local thresholding algorithm of Bernsen (1986), as implemented 652 in ImageJ (Landini 2008; Landini et al. 2016). The window radius was set to a low value 653 (typically on the order of 5 pixels), whilst the contrast threshold was set to a fairly high value 654 (typically in the range of 20-50), so as to reduce the possibility of relatively high density non655 bone material (e.g., dried marrow tissue) from becoming segmented along with the bone phase. 656 The specific values for each parameter varied from specimen to specimen, and were chosen based 657 on iterative visual comparison of the segmented versus unsegmented scans. Subsequent to image 658 segmentation, the scans were 'cleaned' in Mimics with the 'region growing tool', which removed 659 extraneous matter that was included in the segmentation but was not connected to the cancellous 660 bone network. That is, this process removed isolated ('floating') voxels that were not connected 
661 to any adjacent voxels in three dimensions; since cancellous bone is always connected to other

662 bone material, this step did not cause any loss of cancellous bone material (but did remove voxels 663 pertaining to marrow tissue, for example).

664

665 One extant bird femur (Gallus gallus) was found to possess material that was possibly medullary 666 bone; it was processed according to Protocol 1 but only for the femoral head region, where the 667 unidentified material essentially filled all the intertrabecular space and thus was able to be 668 effectively isolated from the cancellous bone. Only the femoral head region was subsequently 669 analysed in this particular specimen.

670

671

672 I.3.2.2 Protocol 2 - extant animal medical CT scans ( $\sim 35$ bones)

673

674 This protocol differs from protocol 1 only in that an additional step was undertaken prior to 675 segmentation. As these scans were acquired with a medical-grade machine, the original dataset 676 was comprised of anisotropic voxels (slice thickness differed from in-plane pixel resolution), 677 which is not ideal for further architectural analyses. Moreover, the scans had a markedly lower 678 resolution compared to the micro-CT scans. As a result, protocol 1 would not result in accurate 679 segmentation of the cancellous structure. To negotiate this problem, the scans were first 680 resampled to triple the original in-plane pixel resolution, and simultaneously resampled along the 681

682 axial direction to produce isotropic voxels. The axial resampling factor is given as $3 f$, where

$$
f=\frac{\text { slice thickness }}{\text { pixel resolution }} \text {. }
$$

683 684

685

686

687

688

689

690

This was performed in ImageJ using 3-D bicubic interpolation, after which the scans were processed according to protocol 1.

The above process of resampling the CT scans is simply to facilitate a more complete and accurate extraction of cancellous bone, without altering the underlying structure in the scan data (Fig. 9). Despite a relatively low resolution to the scans, each individual trabecula was still visible to the naked eye, owing to the partial volume effect (Ketcham \& Carlson 2001; Ryan \& Ketcham 2002b). Here, pixels with intermediate grey-values reflect a volumetric averaging of the 
691 high grey-values of bone and the low grey-values of intertrabecular spaces, and are interpreted by

692 the observer as reflecting intertrabecular spaces. However, as these intermediate-valued pixels are 693 adjacent to high-valued (bone) pixels, they may not be recognized as reflecting non-bone material 694 by a local segmentation algorithm because of insufficient contrast, and so become included in the 695 segmented bone phase, producing an erroneous result. By resampling the CT scans to a higher 696 resolution, the underlying structure in the data is retained, but the number of pixels associated 697 with each phase (bone and non-bone) of each part of the structure is increased (Fig. 9C). This 698 increases the ability of a local thresholding algorithm to distinguish the two phases from each 699 other, as there is higher local contrast, resulting in a more accurate segmentation. Theoretically, 700 the greater the degree to which the scan is resampled, the higher the accuracy of the resulting 701 segmentation. However, there will be a limit to the degree of resampling, beyond which any 702 further information extracted is not genuine, and is artefactual; furthermore, greater resampling

703

704

705

706

707

708

709

710

711

712

713

714

715

716

717

718

719

721

\section{7}

720 Owing to a maximum capable peak tube voltage of $80 \mathrm{kV}$ in the machine used, the resulting produces larger scan datasets, increasing computational requirements for all successive steps in image processing and analysis. Experience has shown that, with the current scan dataset at least, a resampling factor of three was sufficient to achieve accurate segmentation.

\section{I.3.2.3 Protocol 3 - human CT scans (3 bones)}

The human bone scans were collected using peripheral quantitative CT scanning; as with the medical CT scans, the resulting scans often comprised anisotropic voxels. Since the in-plane resolution was always lower than the slice thickness (if the two were not equal), the scans were first resampled in the in-plane directions using bicubic interpolation in ImageJ, resulting in an isotropic image stack. Next, a low-radius 3-D median filter of kernel radius 2 or 3 pixels was applied, again in ImageJ, to remove high-frequency noise in the data (Ollion et al. 2013). Finally, the steps outlined in protocol 1 were followed.

18 I.3.2.4 Protocol 4 - non-avian theropod micro-CT scans (15 bones) scans of the fossil bones suffered from both high-frequency (i.e., short wavelength) and low 
722 frequency (i.e., long wavelength) background noise that overprinted the actual structure in the

723 images. The former was manifest as speckled, 'salt and pepper' patterns, whilst the latter

724 stemmed from scanning artefacts such as beam hardening (Ketcham \& Carlson 2001) and

725 differences in bulk density throughout a specimen. These two sources of noise were removed in

726 Image $\mathrm{I}$ in the following manner, and the process is illustrated in Fig. 10. First, a low-radius 3-D

727 median filter was applied to the original image stack, removing the high frequency noise; the

728 kernel radius used varied between 2-10 pixels, depending on the specimen. Secondly, a large-

729 radius $2 \mathrm{D}$ median filter was successively applied to the original image stack in all three directions

$730(x, y$ and $z)$, to isolate the low-frequency noise; the kernel radius used was large in comparison to

731 the trabecular thickness, and varied between 10-40 pixels, depending on the specimen. A single

732 application of a large-radius 3-D median filter could have been used instead to obtain the same

733 effect, but owing to the larger number of calculations required and large kernel radius, it was

734 prohibitively slow. The low-frequency filtered image stack was then subtracted from the high-

735 frequency filtered stack. That is, this process removes the high-frequency noise, and then

736 removes the background noise. Whilst the resulting image stack was devoid of noise and captured

737 the structure in the data, it nevertheless was texturally 'rough'. Consequently, a low-radius 3-D

738 mean filter, of kernel radius 2 or 3 pixels, was applied to remove this roughness. As this filter was

739 of a high frequency compared to the structure itself, this step did not alter the structure in the data

740 (e.g., trabeculae were not removed, connections did not disappear). Lastly, a global segmentation,

741 with a high-pass grey-value threshold set to 1 or 2, was applied to extract the cancellous bone

742 structure.

743

744 It is important to note that the above approach did not result in the total removal of matrix from

745 the medullary cavity, because the filtering parameters were chosen based on the results for

746 regions of the specimen entirely occupied by cancellous bone. This did not pose a problem to

747 analysis, however, because the remaining noise was essentially random and isotropic, and thus

748 easily distinguished visually from the cancellous bone structure elsewhere. It was therefore easy

749 to avoid the medullary canal in the architectural analyses.

750

751

752 I.3.2.5 Protocol 5 - non-avian theropod medical CT scans ( $\sim 30$ bones) 
754 As with the other set of medical CT scans, the scans of the fossil non-avian theropod bones 755 comprised anisotropic voxels. Moreover, the resolution of the scans, and spatial variation in 756 density and preservation quality, prohibited the proper segmentation of the cancellous bone.

757 Consequently, the scans were resampled in the axial direction only, to produce isotropic voxels;

758 they were not resampled to a higher resolution, as per protocol 2 . This also meant that the 759 cancellous bone architecture was only able to be analysed qualitatively. One exception to this was 760 the CT scans of the Masiakasaurus femora collected by Farke \& Alicea (2009). Owing to the 761 relatively high scan resolution and good preservation of the fossil bones, these scans were able to 762 be processed as per protocol 2, and were subjected to quantitative analyses.

763

764

765

766

767

768

769

770

771

772

773

774

775

776

777

778

779

780

781

782

783

\section{I.3.3 Architectural analyses}

As the resolution and quality of the CT scans varied, several analytical techniques were used. In their own way, each helped to identify the dominant architectural direction in a given region of cancellous bone, and how this varied throughout an entire bony element. Quantitative fabric analysis (Section I.3.3.1) was possible for scans of most (85\%) of the extant animal bones, as well as all the fossil specimens imaged using micro-CT scanning, that is, most scans that were processed as per protocols 1-4 above. The remaining scans of extant animals that were unable to be quantitatively analysed (pertaining to small bird bones), were analysed in a qualitative fashion (Section I.3.3.2), as were the scans of extant birds obtained by Farke \& Alicea (2009). The nonavian theropod scans processed as per protocol 5 above were only able to be analysed in a qualitative fashion; the scans of Masiakasaurus were able to be analysed quantitatively. A subset of the results from the quantitative analyses were also subject to additional analysis to obtain further insight into two key regions of the femur, the femoral head and medial condyle (Section I.3.3.3). Lastly, the architecture of diaphyseal cancellous bone in birds was analysed in a semiquantitative fashion, via a categorical scoring method (Section I.3.3.4). 
785 Quantitative analyses of cancellous bone architecture were conducted using the software

786 Quant3D 2.3 (Ketcham \& Ryan 2004; Ryan \& Ketcham 2002a; Ryan \& Ketcham 2002b). The 3-

787 D fabric tensor (Cowin 1986; see also Fig. 2) for a given volume of cancellous bone was

788 calculated from the segmented CT scans using the star volume distribution method (Cruz-Orive

789 et al. 1992; Odgaard 1997; Odgaard 2001). This is a stereological technique that expresses how

790 cancellous bone material is distributed in three dimensions, and the results of this approach have

791 previously been demonstrated to show strong correlation with cancellous bone mechanical

792 properties (Kabel et al. 1999; Odgaard et al. 1997; Turner et al. 1990; Ulrich et al. 1999). To

793 examine how the fabric varied spatially throughout a given bone, analyses were conducted on

794 numerous (sometimes > 700) discrete volumes of cancellous bone; each volume of interest (VOI)

795 was spherical in shape, to avoid corner effects. The diameter of the VOIs was typically 5 or more

796 times the mean trabecular spacing for a specimen, which is sufficiently large for the continuum

797 assumption of cancellous bone behaviour to hold (Cowin 2001; Harrigan et al. 1988), thus

798 permitting the calculation of a fabric tensor.

799

800 Volumes of interest were arranged in the geometric pattern of cubic close-packed spheres (Fig.

801 11). This arrangement maximizes the volume of cancellous bone analysed, and as no overlap

802 occurs between adjacent spheres, each part of cancellous bone was analysed only once. Hence,

803 unlike the results of Saparin et al. (2011), the results for each VOI were independent of one

804 another. The close-packed pattern was generated in the computer- aided design software

805 Rhinoceros 4.0 (McNeel, USA) and scaled and fitted to each specimen as appropriate. By altering

806 the VOI positions relative to the whole bone in some test specimens, it was found that the exact

807 location of the VOIs did not influence the overall results. That is, the fabric vector field changed

808 gradually enough throughout a bone such that the exact location of VOIs did not alter the results,

809 and no part of the pattern of change was missed. Great care was taken to ensure that peripheral

810 VOIs included at most only minimal amounts of cortical bone or the medullary cavity. This was

811 achieved by manually removing VOIs that did not fall into cancellous bone regions, using 3-D

812 geometric models of each specimen in Rhinoceros (Fig. 11). Post-analysis inspection revealed

813 that inadvertently including small amounts of cortical bone or medullary cavity did not alter the

814 overall results. In the fossil specimens, cracks or other regions of obvious deformation (e.g., 
815 twisting, bending, flattening) were avoided (Bishop et al. 2017); they were identified and their 816 geometry subsequently mapped out in the CT scans using Mimics, and the resulting geometry 817 was used to remove VOIs from analysis in Rhinoceros (Fig. 11).

818

819 For each VOI in a specimen, measurements were made for 2049 uniformly distributed 820 orientations at 4,000 points within the bone phase, with random rotation and dense vector 821 sampling (Ketcham \& Ryan 2004). These were batch processed in Quant3D, controlled using a 822 custom script in MATLAB 8.0 (MathWorks, Natick, USA). For each VOI, Quant3D calculated 823 the fabric tensor, of which the three principal directions ( $\mathbf{u}_{1}$, primary; $\mathbf{u}_{2}$, secondary; $\mathbf{u}_{3}$, tertiary) 824 corresponded to the principal fabric directions of cancellous bone in that particular VOI (i.e., the 825 principal directions of alignment). Additionally, the eigenvalues of this tensor $\left(e_{1}, e_{2}, e_{3}\right)$ 826 expressed the degree to which the cancellous bone was aligned to each of these principal 827 directions. These parameters that described the fabric tensor were extracted from the Quant3D 828 outputs for each VOI using a custom MATLAB script, and were plotted and visualized in 3-D 829 relative to the whole bone using another custom MATLAB script. The vector results were also 830 further visualized and interrogated with respect to the whole bone in Rhinoceros.

\section{I.3.3.2 Qualitative analyses}

837

838 There were a few bird specimens that were too small (especially at their distal ends) for the quantitative architectural analyses described above to be implemented. This was due to their small size, especially for the distal ends. Specifically, their bones were so small, and the trabeculae spaced far enough apart, that no VOI could be placed in the bone which would span at least five intertrabecular lengths. That is, the continuum assumption of cancellous bone behaviour (Cowin 2001; Harrigan et al. 1988) would not hold, preventing a quantitative fabric analysis. For

844 these specimens, cancellous bone architecture was qualitatively assessed by 3-D visualization of 845 the segmented CT scans using isosurface and volume renderings in Mimics and ImageJ, 
846 facilitating visual assessment of the predominant orientation of trabeculae in the different regions

847 of a bone. This approach was also used for the scans of extant bird bones collected by Farke \& 848 Alicea (2009), which had relatively low resolution or high voxel anisotropy. Additionally, 3-D

849 visualization was performed on other specimens where quantitative architectural analysis was

850 possible, to provide further insight into their architecture. Importantly, this also demonstrated that

851 the physical morphologies observed by 3-D visualization correlated with the fabric directions

852 calculated via quantitative analyses: the dominant orientation of trabeculae as assessed visually

853 largely coincided with the primary fabric direction.

854

855 For the non-avian theropod specimens imaged using medical CT scans, 3-D visualization and 856 assessment of cancellous bone architecture used the Volume Viewer 2.0 plugin for ImageJ 857 (Barthel 2006). This allowed real-time re-slicing and racking of a CT scan image stack in any 858 orientation. Not only did this permit visualization of the scans from any direction, it also helped 859 identify architectural features that were difficult to see in any single static slice, but which were 860 revealed upon dynamic racking through successive slices. It also allowed for the identification of 861 scanning artifacts (Ketcham \& Carlson 2001), and hence these could be ignored from 862 assessments of architectural patterns. Based on these visualizations, the observed patterns were 863 mapped onto whole-bone models via geometric representations of the dominant architectural 864 patterns and directions, using a combination of spline curves and deformable surfaces in 865 Rhinoceros. The accuracy of these geometric models was assessed through constructing multiple 866 cutting planes through the model in different orientations, and comparing the cut model geometry 867 to the CT slices in the same orientation. The geometric models were iteratively developed and 868 refined as appropriate until good visual agreement between the scan data and the model was 869 achieved. In using these geometric models, it is implicitly assumed that they accurately capture 870 the dominant fabric directions in a given region of cancellous bone. All qualitative assessments 871 were initially undertaken by a single person (PJB), with later conferral provided by the other 872 authors. To help illustrate the above procedure for the reader, an example femur CT scan dataset 873 and the resulting geometric model is freely accessible from a Figshare repository

874 (10.6084/m9.figshare.c.4224926). The scans and models can be visualized using the open source 875 softwares ImageJ and Meshlab (http://meshlab.sourceforge.net/), respectively. 876 
$878 \quad$ I.3.3.3 Anatomically explicit analyses of femora

879

880 The architectural directions identified by the quantitative and qualitative analyses above

881 were largely interpreted in a qualitative fashion, in the context of whole-bone general osteology

882 and spatial variation in cancellous bone fabric (i.e., how they varied throughout 3-D space).

883 However, strict quantitative focus was also directed towards the primary fabric direction of

884 cancellous bone in the femoral head and medial femoral condyle, referenced to an explicit

885 anatomical coordinates system defined by osteological landmarks. As extant birds employ a

886 subhorizontal femoral posture, while most, if not all, non-avian theropods have been

887 hypothesized to have employed a subvertical femoral posture (e.g., Carrano 1998; Farlow et al.

888 2000; Gatesy 1991b; Gatesy et al. 2009; Hutchinson 2006; Hutchinson \& Allen 2009), it is

889 logical that this may be reflected in the architecture of cancellous bone in these two regions. This

890 correspondence is possible because flexion of the hip joint determines the degree of femoral

891 crouch, and flexion of the knee joint will vary with femoral crouch such that the feet remain

892 underneath the whole-body COM (Hutchinson \& Allen 2009). To assess this conjecture, the

893 mean orientation of the primary fabric vector in the femoral head and medial femoral condyle

894 was determined for each specimen (except for the crocodylians and varanids, which are

895 sprawling quadrupeds) using a custom MATLAB script, by calculating the vectorial mean of the

896 primary fabric vectors for all VOIs in the relevant region (Allmendinger et al. 2013). The mean

897 fabric direction was then referenced in a bone anatomical coordinate system, which was defined

898 in a consistent manner across all species (Fig. 12). This anatomical coordinate system was based

899 on two spheres fitted to the distal condyles, calculated using 3-Matic 9.0 (Materialize NV,

900 Belgium), and the principal axis of inertia of the whole bone, calculated for a surface mesh of the

901 bone using Meshlab 1.3.3. The $z$-axis was defined by the principal axis of inertia (i.e., the long

902 axis of the bone; $+z$ is proximal); the $y$-axis defined as the cross-product of the $z$-axis and the

903 vector joining the centres of the two condyle spheres ( $+y$ is anterior); and the $x$-axis defined as the

904 cross-product of the $y$ - and $z$-axes ( $+x$ is lateral). The anatomically referenced mean fabric

905 directions were then assessed using a stereographic projection (stereoplot) of the data (Amson et

906 al. 2017; Ryan \& Ketcham 2005).

907 
$909 \quad$ I.3.3.4 Diaphyseal cancellous bone analyses

910

911 In both the bird and other reptile specimens investigated, a significant amount of cancellous bone

912 was frequently observed to encroach from the ends of the bone in to the diaphysis (shaft), in both

913 the femur and tibia. In the regions nearer the ends, the amount was sometimes sufficient enough

914 that quantitative fabric analyses could be undertaken, particularly in the bones of larger species.

915 Usually, however, too little cancellous bone was present to permit such quantitative analysis.

916 Nevertheless, throughout the course of 3-D visualization, these bones were observed to show a

917 variety of interesting patterns, and so an attempt was made to characterize the variation in

918 architectures in the bird bones, by categorical scoring.

919

920 Categorical scoring was performed by five independent, volunteer observers (non-scientists who

921 had varying levels of experience with vertebrate anatomy), who were blind to the objectives of

922 the present study. They were asked to inspect the same 3-D isosurface models of each bone,

923 derived from the segmented CT scans, assess them for three features and score each bone on a

924 pre-defined, categorical scale for each feature. The first feature was the bulk spatial extent of

925 cancellous bone in the diaphysis, with scores assigned on a four-point scale: $0=$ essentially the

926 whole diaphysis was void of cancellous bone, $1=$ less than half of the diaphysis was occupied by

927 cancellous bone, 2 = half or more than half of the diaphysis was occupied by cancellous bone, $3=$

928 essentially the whole diaphysis was occupied by cancellous bone. The second feature was the

929 average orientation of trabeculae with respect to the long axis of the shaft, with scores assigned

930 on a nine-point scale of $10^{\circ}$ increments (Fig. 13A): $0=0$ to $10^{\circ}, 1=10$ to $20^{\circ}, 2=20$ to $30^{\circ}$, and

931 so on. The third feature was the tendency of trabeculae to be closely associated with other

932 trabeculae, with scores assigned on a three-point scale (Fig. 13B): $0=$ trabeculae mostly or

933 always occur singly, well separated from any other trabeculae; 1 = trabeculae occur in small

934 groups of two or three other, similarly situated trabeculae; 2 = trabeculae mostly or always occur

935 in close association with many other similarly situated trabeculae. If a given bone was scored as

936 ' 0 ' for the first feature, then it was not scored for the second or third ('n/a').

937 
938 Following scoring, the mean score across the five observers was taken for each bone and for each

939 morphological feature. For the second and third features, a given bone was sometimes scored as

940 ' $\mathrm{n} / \mathrm{a}$ ' (if the first feature was scored as a ' 0 '), and other times it was given an actual numeric

941 score. If there were more ' $\mathrm{n} / \mathrm{a}$ ' scores than numeric scores assigned for a given specimen, then the

942 mean score was taken as ' $\mathrm{n} / \mathrm{a}$ ', and that particular specimen did not contribute towards further

943 analyses (detailed below). However, if the majority of scores assigned were numeric, then the

944 mean score was taken as the mean of those numeric scores.

945

946

947

\section{I.3.4 Statistics}

948

949 Three datasets were examined to test for a relationship between measured parameters and body

950 size in birds: the anterior inclination of the mean fabric direction in the femoral head, the

951 posterior inclination of the mean fabric direction in the medial femoral condyle, and the results of

952 categorical scoring of diaphyseal cancellous bone. The length of the relevant bone was taken here

953 as a proxy for body size, and was measured as the total proximal distance between articular

954 condyles (interarticular length), excluding crests and trochanters. Bone length was used here as it

955 has direct mechanical relevance to the loads a bone experiences (e.g., it determines lever arms for

956 bending moments); additionally, using bone length precludes any margin for error that is

957 attendant with the use of other body size measures, such as estimated body mass. A relationship

958 with body size would normally be assessed using a standard parametric test of the slope of a

959 major axis regression (Warton et al. 2006). However, the data often exhibited non-normal

960 distribution of errors (non-normal probablility plot) and heteroscedasticity (Breusch-Pagan test),

961 as determined in PAST 3.09 (Hammer et al. 2001). As such, a parametric test could not be

962 implemented. Instead, a permutation test of the slope with 100,000 replicates was used,

963 implemented in a custom MATLAB script (Legendre \& Legendre 2012); the significance level

964 was set at $p=0.05$.

965

966 In addition, the reliability of the scorers in the analysis of diaphyseal cancellous bone was

967 assessed using the intraclass correlation coefficient (ICC). This was calculated in PAST 3.09,

968 using the $\operatorname{ICC}(2, k)$ model of (Shrout \& Fleiss 1979), where $k$ in this instance is 5.The higher the 
969 value of the ICC, the more reliable the scorers. The ICC was only able to be calculated for bones

970 that had received numeric scores by all five scorers. Furthermore, for features 2 and 3 of the

971 tibiotarsus, an ICC could not be calculated, because there was only one specimen in each set that

972 had received a numeric score by all five scorers. That is, for every other specimen, at least one

973 scorer had scored feature 1 as ' 0 ', and then assigned the ' $\mathrm{n} / \mathrm{a}$ ' score to the remaining two features.

974

975

976 I.4 Results

977

978 Since the architecture of cancellous bone in all but one of the species investigated here (humans)

979 has never been studied before, the results of this study are first and foremost descriptive,

980 qualitatively characterizing the whole-bone architectural patterns. Where quantitative data was

981 produced, it is presented where appropriate, including graphs, stereographic plots and vector field

982 plots as well as reporting of numerical results (including statistics when possible); elsewhere,

983 qualitative observations are presented using geometric models and isosurface renderings of bones

984 in cross-section, in concert with verbal anatomical description. The presentation of data and

985 observations is organized primarily by each bone investigated: the femur in Section I.4.3.1, the

986 tibia (tibiotarsus) in Section I.4.2 and the fibula in Section I.4.3. For each bone, results are

987 presented in sequential order, first with humans and birds (extant bipeds), then other extant

988 reptiles (theropod outgroups) and finally the non-avian theropods. Overall, birds were quite

989 consistent in their observed architectural patterns, across all species studied, and it is therefore

990 convenient to treat all species together, with specific differences noted where appropriate. The

991 observations for non-avian theropods are presented in approximate phylogenetic order (i.e.,

992 clades progressively closer to crown-group birds). Furthemore, the observations reported for a

993 given group pertain to all specimens studied for that group, unless otherwise indicated. It should

994 also be noted that since this study was undertaken, a recent re-evaluation of North American

995 troodontid taxonomy has cast doubt on the validity of the name Troodon formosus (van der Reest

$996 \&$ Currie 2017). As such, the troodontid material investigated here will be referred to as

997 'Troodontidae sp.', pending further study.

998

999 
1000

1001

1002

1003

1004

1005

1006

1007

1008

1009

1010

1011

1012

1013

1014

1015

1016

1017

1018

1019

1020

1021

1022

1023

1024

1025

1026

1027

1028

1029

1030

\section{I.4.1 Femur}

\section{I.4.1.1 General remarks}

Observations for the single human femur studied are consistent with previously published reports (e.g., Elke et al. 1995; Garden 1961; Koch 1917; Singh et al. 1970; Takechi 1977; Tobin 1955; Townsley 1944; von Meyer 1867), supporting the use of this specimen as a general reference for humans. Cancellous bone occurs throughout both proximal and distal ends, but the majority of the diaphysis is devoid of it. However, cancellous bone is present deep to and in the immediate vicinity of the lesser trochanter on the proximal shaft, which is distinct from and non-homologous with the lesser trochanter of theropods.

Cancellous bone is more extensive in the birds and other reptiles than humans. It usually encroaches into the diaphysis to a varied, and often large, extent, although moving towards midshaft it tends to become progressively more sparse and restricted to the endosteal margin of the cortex. A more detailed treatment of diaphyseal cancellous bone is given below (Section I.4.4). Pneumatized bird femora - easily distinguished by the presence of pneumatopores - are distinctly more 'loose-packed' compared to marrow-filled bird femora; that is, pneumatized bones have greater trabecular spacing, a distinction that exists in both small and large species (Fig. 14). Despite the modulating effect that pneumatization has on trabecular spacing, no effect on the patterns of fabric direction was evident.

Similar to humans, in non-avian theropod femora the proximal and distal ends are fully occupied by cancellous bone, but the majority of the diaphysis remains empty. As with the human lesser trochanter, cancellous bone also occurs locally deep to the fourth trochanter, although this was only observed in the larger species, where the fourth trochanter is prominently developed (both on account of bone size and phylogenetic position: Gatesy 1990; Hutchinson 2001a). In larger femora, cancellous bone can encroach further into the diaphysis, with a limited number of thick trabeculae extending from the metaphysis along the endosteal surface. This encroachment is most extensive in Tyrannosaurus femora, although the middle third to half of the bone still remains free of trabeculae. It is likely that this size-dependency of diaphyseal encroachment by cancellous 
1031 bone is purely a function of allometry: in longer femora, the ends are larger and the diaphysis is 1032 relatively shorter in length.

1033

1034

1035

\section{I.4.1.2 Proximal femur}

1036

1037

1038

In the human proximal femur, the primary fabric direction $\left(\mathbf{u}_{1}\right)$ in the head is essentially proximodistally oriented, with a gentle anteromedial inclination of about $5^{\circ}$ from the proximodistal axis, which grades into a stronger medial inclination in the distal (inferior) part of the femoral neck (Fig. 15A). This corresponds to the widely recognized 'primary compressive group' noted in previous studies of humans (see also Fig. 4A), as well as other primates (Rafferty 1998; Scherf 2008). In the region of the greater trochanter (which is distinct from and nonhomologous with the greater trochanter of theropods), $\mathbf{u}_{1}$ largely parallels the lateral margin of the trochanter, corresponding to the 'greater trochanter group' of previous studies (Fig. 15A). Within the main part of the metaphysis, a double-arcuate pattern of $\mathbf{u}_{1}$ occurs in the coronal plane (or more accurately, the plane containing the shaft and femoral neck), where it arcs from opposite sides of the metaphysis to intersect in the middle. Moreover, the secondary fabric direction $\left(\mathbf{u}_{2}\right)$ in this region is also largely contained within (parallel to) the coronal plane. This double-arcuate pattern corresponds to the 'secondary compressive' and 'secondary tensile' groups of previous studies (Fig. 15B). The orientation of $\mathbf{u}_{1}$ in the distal metaphysis, underneath the lesser trochanter and in the transition to the diaphysis is subparallel to the long-axis of the bone (Fig. 15C).

1052

The proximal femur of birds shows the same general pattern irrespective of size. In the femoral head, $\mathbf{u}_{1}$ is mainly proximodistally oriented, but there tends to be a variable degree of anteromedial inclination superimposed upon this (Fig. 16A-D). The anterior component of this inclination is often quite pronounced, ranging up to $60^{\circ}$ from the proximodistal axis. This anteromedial inclination continues down into the distal femoral neck as well. Under the facies antitrochanterica, $\mathbf{u}_{1}$ is largely proximodistally oriented, but often there is also a gentle posteromedial inclination (Fig. 16E-I). This orientation continues towards the trochanteric crest, where the medial and posterior inclination often becomes more pronounced (Fig. 16J-N). However, in the anteriormost part of the trochanter, there can sometimes be no posterior 
1062 inclination at all. Progressing distally down the metaphysis, beyond the distal level of the femoral 1063 head and into the diaphysis, the orientations of $\mathbf{u}_{1}$ become more 'disorganised': they take on a 1064 more oblique orientation relative to the long-axis of the bone, and the change in direction across

1065 the bone is no longer a gradual transition (Fig. 16O,P). No double-arcuate pattern of $\mathbf{u}_{1}$ and $\mathbf{u}_{2}$, as 1066 described above for the human femur, was observed in any bird specimen. Moreover, $\mathbf{u}_{2}$ is 1067 generally subparallel to the axial plane, and in large bones they are also parallel to the bone 1068 periphery, forming a concentric pattern. Progressing distally through the metaphysis, however, 1069 the orientations of $\mathbf{u}_{2}$ become more obliquely oriented and disorganized, as with the orientations $1070 \mathbf{u}_{1}$. Although fabric orientation could not be extensively quantified in the smaller bird femora, 1071 visualization of isosurface and volume renderings of the bones themselves shows that for a given 1072 region, the trabeculae are oriented in the same general direction as the $\mathbf{u}_{1}$ for larger bird femora 1073 (Fig. 16I, N).

1074

In the extant sprawling reptile femora examined, the orientation of $\mathbf{u}_{1}$ is subparallel to the long1076 axis of the bone throughout the metaphysis (Fig. 17A-D), except in the region of the fourth 1077 trochanter, where it is largely parallel to the long-axis of the trochanter itself (Fig. 17B,D). 1078 Leading up to the head region, $\mathbf{u}_{1}$ fans out, away from the metaphysis and towards the articular 1079 surface (Fig. 17A-D). In the distal metaphysis and transitioning to the diaphysis, the orientation 1080 of $\mathbf{u}_{1}$ progressively becomes more obliquely oriented and disorganized, in the same fashion as the 1081 bird femora (Fig. 17E).

1082

1083 Only limited information could be gleaned for the proximal femur of the small non-avian 1084 theropod Masiakasaurus, owing to the small size of the specimens. The orientation of $\mathbf{u}_{1}$ 1085 throughout the proximal end is more or less proximodistally directed, leading from the base of 1086 the femoral neck up to the apex of the head (Fig. 18). A gentle medial inclination from the 1087 proximodistal axis is present in most specimens.

The proximal femur of both Allosaurus and the tyrannosaurids show a strikingly similar pattern to that of humans (Fig. 19A-E). There is a well-developed tract of dense, cancellous bone extending from the base of the femoral neck up towards the apex of the head of the femur, much 1092 like the 'primary compressive group' of humans (Fig. 19A-E, maroon). As in humans, too, this 
1093 tract has a gentle anterior inclination relative to the proximodistal axis (Fig. 19F). Additionally, a 1094 double-arcuate pattern is evident in the tyrannosaurids, also similar to the human pattern. The 31095 D visualization of CT scans suggests that whilst this pattern is most developed in the coronal 1096 plane, it does extend out of that plane somewhat, with the 'sheets' of trabeculae being partially 1097 concentric with the bone's periphery (Fig. 19A-E, maroon and green). A second double-arcuate 1098 pattern is also present in the lesser trochanter, again subparallel to the coronal plane (Fig. 19A-E, turqoise and purple). A modest quantity of cancellous bone is present in the fourth trochanter, and the dominant direction is directed posteroproximally, parallel to the distal margin of the trochanter (Fig. 19A-E, blue). In the distal metaphysis all observed cancellous bone (and sometimes individual trabeculae) is oriented subparallel to the bone's long-axis; there is no indication of marked obliquity or disorganization as seen in the bird and other reptile femora.

In the femoral head of ornithomimids, the most conspicuous feature is that $\mathbf{u}_{1}$ is oriented predominantly in an anteroposterior direction, yet $\mathbf{u}_{2}$ is oriented proximodistally with a gentle medial inclination (directed towards the apex of the head), much like the orientation of $\mathbf{u}_{1}$ in the femoral head of humans, birds and (presumably) Allosaurus and the tyrannosaurids (Fig. 20AD). In fact, a generally proximodistal orientation of $\mathbf{u}_{2}$ and a generally anteroposterior orientation of $\mathbf{u}_{1}$ also occurs throughout much of the proximal end of the femur. Only around the anterior and posterior peripheries of the bone does $\mathbf{u}_{1}$ assume the more typical proximodistal orientation, with a medial inclination in the region of the femoral head and neck. It also assumes a more proximodistal orientation progressing towards the greater trochanter and distal end of the metaphysis, but in the latter region is still shows a marked level of 'disorganization' (Fig. 20E). Along the anterior part of the lesser trochanter, $\mathbf{u}_{1}$ is aligned more or less parallel with the anterior margin in a generally proximodistal fashion (Fig. 20F). Further posteriorly into the main body of the trochanter, however, it takes on a more mediolateral orientation, which gradually becomes more anteroposteriorly directed as the trochanter merges with the main body of the proximal femur.

The single caenagnathid femur examined exhibits a pattern very much like that in the 1122 ornithomimids. The only notable difference occurs in relation to the lesser trochanter, which has 1123 decreased in size and become more unified with the greater trochanter. Here, the fabric pattern is 
1124 essentially a continuation of what occurs elsewhere in the head and metaphysis, with $\mathbf{u}_{1}$

1125 becoming anteromedially oriented, but still subparallel to the axial plane (Fig. 20G). The

1126 orientation of $\mathbf{u}_{2}$ remains predominantly proximodistal, although a small posterior component is

1127 also present.

1128

1129

In the therizinosauroid Falcarius, the spatial pattern of the orientation of $\mathbf{u}_{1}$ is largely comparable

1130 to that observed in humans, throughout the whole proximal end of the femur, save the lesser trochanter. In the head, $\mathbf{u}_{1}$ has a slight anteromedial component superimposed over an otherwise predominantly proximodistal orientation, which is directed toward the apex (Fig. 21A). In the middle of the metaphysis, $\mathbf{u}_{1}$ exhibits a weakly developed double-arcuate pattern that is parallel to the coronal plane (Fig. 21A). Additionally, in the distal metaphysis $\mathbf{u}_{1}$ is largely subparallel to the long-axis of the bone, showing little 'disorganization' as seen in the birds. In the lesser trochanter, both $\mathbf{u}_{1}$ and $\mathbf{u}_{2}$ are largely contained within the coronal plane. Towards the base of the trochanter, $\mathbf{u}_{1}$ is generally oriented proximodistally, and $\mathbf{u}_{2}$ is generally oriented mediolaterally; however, nearer the apex, $\mathbf{u}_{1}$ is generally oriented mediolaterally, and $\mathbf{u}_{2}$ is generally oriented proximodistally (Fig. 21B,C). One distinct difference to the pattern observed in humans is the orientation of $\mathbf{u}_{2}$ in much of the proximal end; here, it is anteroposteriorly aligned, much like $\mathbf{u}_{1}$ in the ornithomimid femora (Fig. 21D).

In the proximal femur of Troodontidae sp., the orientation of $\mathbf{u}_{1}$ is predominantly proximodistal. Relative to the proximodistal axis, it assumes a gentle medial inclination as it courses from the base of the femoral neck up towards the apex of the head; within the head, it also takes on an anterior inclination (Fig. 21E,F). Under the region homologous with the facies antitrochanterica of birds, and in the region of the greater trochanter, $\mathbf{u}_{1}$ has a posteromedial component to its orientation (Fig. $21 \mathrm{G}, \mathrm{H}$ ). In the anterior part of the proximal femur, $\mathbf{u}_{1}$ is largely contained within the coronal plane, but shows little preferred orientation within that plane; $\mathbf{u}_{2}$ is not contained within the coronal plane, unlike in the homologous region of the femur of Falcarius (base of lesser trochanter). In the region of the lesser trochanter, which is small and proximally located in

1152 Troodontidae sp., $\mathbf{u}_{1}$ tends to take on a more mediolateral orientation (Fig. 21I). Progressing more 1153 distally through the metaphysis, the orientation of $\mathbf{u}_{1}$ becomes more disorganized and oblique to 1154 the bone's long axis, as seen in the proximal femur of birds and sprawling reptiles (Fig. 21J). No 
1155 double-arcuate pattern of any form, as observed in humans, Allosaurus, tyrannosaurids or

1156 Falcarius, was observed in Troodontidae sp.

1157

1158 The mean orientation of the primary fabric direction $\mathbf{u}_{1}$ in the femoral head for each specimen 1159 analysed is presented in Fig. 22. The ornithomimid and caenagnathid specimens were excluded, 1160 owing to their distinct fabric architecture (mean fabric directions of $\mathbf{u}_{1}$ are oriented at almost $90^{\circ}$

1161 to the general orientation of what is observed in other species; see also above); additionally, the 1162 femoral head of the caenagnathid specimen was incomplete. A general orientation was taken to 1163 represent Allosaurus and the tyrannosaurids, based on the geometric model developed to 1164 represent their architectural patterns (Fig. 22A, purple; cf. Fig. 22C,D). In almost all specimens, 1165 the mean fabric direction is oriented anteromedially. The human and Masiakasaurus specimens 1166 plot close to the pole of the stereoplot, indicating that their mean directions are oriented almost 1167 purely proximodistally. The plot for Falcarius and the general Allosaurus-tyrannosaurid pattern 1168 is a little further from the pole (more medially directed), but still has little anterior inclination.

1169 The bird results demonstrate a sizeable degree of spread in the northwest quadrant of the plot, and 1170 indeed variability occurs within the species or genera for which multiple individuals were studied 1171 (Fig. 22B). However, on the whole, birds exhibit a substantial anterior inclination to the mean 1172 fabric direction; the mean direction across all birds is inclined $21.6^{\circ}$ anterior of the proximodistal 1173 axis in the sagittal plane. Major axis regression of the bird data set revealed that the sagittal 1174 inclination of the mean fabric direction did not vary significantly with femur length (slope = 11750.059 , intercept $\left.=58.4, r^{2}=0.100, p=0.108\right)$. The mean primary fabric direction in Troodontidae $1176 \mathrm{sp}$. is of an intermediate orientation between that of birds and the other non-avian theropods, 1177 having an anterior inclination in the sagittal plane of $15.8^{\circ}$.

\section{I.4.1.3 Distal femur}

Throughout the distal femur of the human specimen, $\mathbf{u}_{1}$ is predominantly oriented subparallel to the long axis of the bone. In the central part of the metaphysis, $\mathbf{u}_{1}$ exhibits a double-arcuate pattern in both the sagittal and coronal planes, with the individual fabric vectors tending to arc 
1186 pattern is not as strongly developed as in the proximal femur. In both the medial and lateral

1187 condyles, $\mathbf{u}_{1}$ has a largely proximodistal orientation (Fig. 23C). Additionally, $\mathbf{u}_{2}$ is largely parallel

1188 to the axial plane, and two prominent tracts or trajectories are evident, one in each condyle (Fig.

1189 23D). These tracts, noted in previous studies (e.g., Takechi 1977) arc from the anterior aspect of

1190 their respective condyle back towards the posterior aspect, and together they form a distinctive

1191 'butterfly pattern'.

1192

1193 In birds, the distal femur exhibits a fairly consistent set of patterns across species. The orientation 1194 of $\mathbf{u}_{1}$ in the metaphysis is largely proximodistally oriented. In the central metaphysis of larger

1195 birds, for which substantial quantification of fabric direction was possible, $\mathbf{u}_{1}$ exhibits a

1196 moderately developed double-arcuate pattern in the sagittal plane between the medial and lateral

1197 condyles, much as in humans (Fig. 24A). Also as observed in humans, the orientation of $\mathbf{u}_{2}$ in the

1198 condyles forms a butterfly pattern in the axial plane, or more correctly, the plane that passes

1199 through the centres of the condyles (Fig. 24B-D). Unlike humans, however, the orientation of $\mathbf{u}_{1}$

1200 in the condyles often has a marked posterior inclination relative to the proximodistal axis,

1201 although it generally remains subparallel to the sagittal plane (Fig. 24E-K). Moreover, $\mathbf{u}_{1}$ sweeps

1202 a distinctly wide arc in the sagittal plane (often in excess of $100^{\circ}$ ), such that in the posterior and

1203 posterodistal extremities of the condyles, $\mathbf{u}_{1}$ can be perpendicular to the proximodistal axis. In

1204 large birds, this sweeping can also extend into the anterior parts of the condyles, where $\mathbf{u}_{1}$ is

1205 anterodistally directed (Fig. 24F). In the condyles of small bird femora for which only limited

1206 quantitative analysis was possible, both $\mathbf{u}_{1}$ and $\mathbf{u}_{2}$ are subparallel to the sagittal plane, and $\mathbf{u}_{1}$ is

1207 inclined posteriorly. In small bird femora for which quantitative analysis was not possible, much

1208 of the distal end is typically occupied by a small number of large but sparsely dispersed

1209 trabeculae. They vary from rod- to plate-shaped, but generally are parallel to the sagittal plane

1210 (Fig. 24D,K). As with the proximal femur, progressing towards the proximal metaphysis and into

1211 the diaphysis reveals a more oblique and disorganized nature to the individual vectors (Fig. 24L).

1213 In the distal femur of extant sprawling reptiles, the orientation of $\mathbf{u}_{1}$ in the metaphysis is largely

1214 subparallel to the long-axis of the bone. In some specimens $\mathbf{u}_{1}$ becomes more disorganized and

1215 obliquely oriented relative to the bone's long axis progressing towards the diaphysis, but this is

1216 not as pronounced as compared to the proximal femur, or as compared to birds. The orientation 
1217 of $\mathbf{u}_{2}$ in the condyles is largely parallel to the axial plane, and exhibits the butterfly pattern seen in

1218 birds and humans (Fig. 25A,B). The main point of difference from birds arises in the orientation

1219 of $\mathbf{u}_{1}$ in the condyles: whilst $\mathbf{u}_{1}$ is posteriorly inclined in the sagittal plane, it does not display the

1220 large anteroposterior sweeping that is often present in birds, sweeping at most about $40^{\circ}$ (Fig.

1221 25C-F).

1222

1223

As with the proximal femur, only limited information could be gleaned for the distal femur of

Masiakasaurus. The orientation of $\mathbf{u}_{1}$ throughout the distal end is more or less proximodistally

1226

1227 oriented, generally with a slight posterior inclination in the sagittal plane.

In the distal femur of the tyrannosaurids, cancellous bone around the periphery of the metaphysis is oriented subparallel to the long axis of the bone. Additionally, there are two sets of paired, arcuate, sheet-like tracts of cancellous bone, which arc largely in the coronal plane (Fig. 26). The obliquity of these sheet-like tracts appears to change across the bone, such that one set of tracts radiates from the 'patellar' or intercondylar groove at the anterior margin of the bone (Fig. 26, maroon and turquoise), and the other set radiates from the popliteal area at the posterior margin of the bone (Fig. 26, green and purple). This feature was not observed in the Allosaurus specimens studied, for insufficient CT scan contrast or resolution did not reveal any information about the metaphysis. In the medial and lateral condyles of both Allosaurus and the tyrannosaurids, the dominant direction of cancellous bone is largely parallel to the sagittal plane, and parallel to the long axes of the condyles, thus producing a butterfly pattern in axial crosssection. Within the sagittal plane, the dominant direction has a marked posterior inclination relative to the proximodistal axis; it also exhibits some amount of anteroposterior sweeping, about $30^{\circ}$ or so (Fig. 26, red).

In both the ornithomimid and caenagnathid specimens, the orientation of $\mathbf{u}_{1}$ is predominantly oriented subparallel to the long axis of the bone throughout much of the distal femur, particularly anteriorly. In much of the medial and lateral condyles, $\mathbf{u}_{1}$ is largely parallel to the sagittal plane and gently inclined posteriorly (Fig. 27A-D); anteroposterior sweeping in the sagittal plane is limited to about $20^{\circ}$. However, in the posterior parts of both condyles, the posterodistal orientation gradually changes to become nearly perpendicular to the long-axis of the bone, and 
1248 almost perpendicular to the local bone surface around the intercondylar sulcus (Fig. 27A-D,

1249 yellow). That is, in the posterior parts of the condyles, $\mathbf{u}_{1}$ appears to radiate away from the 1250 intercondylar sulcus, largely within the axial plane (Fig. 27E). In these regions, $\mathbf{u}_{2}$ is oriented

1251 largely proximodistally in the sagittal plane, but elsewhere in the condyles $\mathbf{u}_{2}$ forms the butterfly

1252 pattern observed in all other groups (Fig. 27F).

1253

1254

1255

1256

The central part of the Falcarius distal femur studied (UMNH VP 12360) is fractured, so little can be said concerning the metaphysis, except that along the medial and lateral peripheries $\mathbf{u}_{1}$ is oriented largely parallel to the long axis of the bone. In both medial and lateral condyles, the orientation of $\mathbf{u}_{1}$ is gently inclined posteriorly and subparallel to the sagittal plane, with little anteroposterior sweeping evident (Fig. 28A,B). The orientation of $\mathbf{u}_{2}$ in the condyles is subparallel to the axial plane, and forms a typical butterfly pattern.

1260

Throughout much of the distal femur of Troodontidae sp., the orientation of $\mathbf{u}_{1}$ is subparallel to the long-axis of the bone, although in the proximal metaphysis it tends to become more obliquely

1263

1264

1265

1266

1267

1268

1269

1270

1271

1272

1273

1274

1275

1276

1277

1278 oriented and disorganized, as seen in birds (Fig. 28C). No indication of any arcing patterns in $\mathbf{u}_{1}$, as observed in humans and birds, was observed. Moreover, the radiating patterns (originating from the intercondylar sulcus) that were observed in the tyrannosaurids, ornithomimids and caenagnathid are not evident either. The orientation of $\mathbf{u}_{1}$ in the condyles is subparallel to the sagittal plane and gently inclined posteriorly (Fig. 28D,E); as in Falcarius, little anteroposterior sweeping is apparent. As with all other groups, $\mathbf{u}_{2}$ in the condyles is subparallel to the axial plane and forms a butterfly pattern (Fig. 28F).

The mean orientation of the primary fabric direction in the medial femoral condyle for each specimen analysed is presented in Fig. 29A. Note that some of the smallest bird femora could not be analysed here, because they possessed too little cancellous bone to facilitate a quantitative analysis. As for the femoral head, a general orientation was taken to represent Allosaurus and the tyrannosaurids. In most specimens, the mean fabric direction is oriented posteriorly, with a small medial inclination. The human and Masiakasaurus specimens again plot close to the pole of the stereoplot, indicating an almost proximodistal mean direction. The plots for the ornithomimid, caenagnathid, Falcarius and Troodontidae sp. are slightly further from the pole, and the general 
1279 Allosaurus-tyrannosaurid pattern is a little further away again. As in the femoral head, the bird 1280 results demonstrate marked variation, although on the whole a substantial posterior inclination is 1281 present; the mean direction across all birds is inclined $24.7^{\circ}$ posterior of the proximodistal axis in 1282 the sagittal plane. Furthermore, the mean primary fabric direction in smaller birds tends to be 1283 more posteriorly inclined in the sagittal plane compared to that in larger birds, as indicated by 1284 major axis regression (Fig. 29B; slope $=-0.12248, r^{2}=0.3858, p=0.00228$ ).

1285

1286

1287

\section{I.4.2 Tibia or tibiotarsus}

1288

1289

\section{$\underline{\text { I.4.2.1 General remarks }}$}

1290

1291

1292

As with the femur, observations for the single human tibia studied are comparable with

1293 previously published reports (e.g., Takechi 1977; von Meyer 1867), again supporting the use of this specimen as a general reference for humans. Cancellous bone is present throughout the 1294 entirety of both proximal and distal ends; a small amount also extends well into the diaphysis along the endocortical margin, but is mostly only one or two trabeculae thick (see Section I.4.4).

Cancellous bone is again generally more extensive in the birds and other extant reptiles compared to humans, at least in the larger species. It usually encroaches into the diaphysis to a varied extent, although moving towards mid-shaft it becomes more sparse and restricted to the endosteal margin of the cortex. Compared to the femur, the tibiotarsus of birds is generally less invaded by cancellous bone; this is especially true of the smaller species. Indeed, in some of the smaller species of birds, the medullary cavity extends well into the proximal and distal ends, to the point that only a handful of large, well-spaced trabeculae remain, with many smaller trabeculae distributed around the periphery. There is virtually no cancellous bone under the tibiofibular crest in birds, regardless of body size. Rather, the crest mostly comprises thickened (but higher porosity) cortical bone. In those birds with a prominent tibiofibular crest (e.g., Porphyrio, Gallinula, Ardeotis, Threskiornis), some cancellous bone does exist, but trabeculae are still few 1308 in number. 
1310 Owing to the logistical constraints of specimen availability and limited available scan time, the 1311 tibia and proximal tarsals of non-avian theropods were not investigated as thoroughly as they 1312 were for the femur. In those specimens that were studied, the proximal and distal ends of the tibia 1313 are fully occupied by cancellous bone (as is the entirety of the astragalus and calcaneum), but the 1314 majority of the diaphysis remains empty. Proximally, cancellous bone encroaches as far distally 1315 as the beginning of the tibiofibular crest; distally, cancellous bone usually does not occur any 1316 further proximally than the point at which the tibia begins to flare out mediolaterally. In the large 1317 tyrannosaurids, however, the distal diaphysis contains a significant amount of cancellous bone 1318 proximal to the point of mediolateral widening, which is again probably an effect of allometry. 1319 Cancellous bone is also present under the tibiofibular crest for its entire length, with more being present in the larger species.

\section{I.4.2.2 Proximal tibia}

1324

1325

In the human proximal tibia, the primary fabric direction $\mathbf{u}_{1}$ is more or less proximodistally oriented throughout the entire end. Under the medial and lateral tibial condyles it has a slight (approximately $10^{\circ}$ ) posterior inclination relative to the proximodistal axis, and under the medial condyle there is also a slight medial component (Fig. 30). As with the femur of humans, in the distal metaphysis and transition into the diaphysis $\mathbf{u}_{1}$ remains proximodistally oriented, with little disorganization or obliquity.

1331

In birds, the orientation of $\mathbf{u}_{1}$ throughout the proximal tibia is predominantly proximodistal, but there are marked departures from this locally throughout the bone. In the anterior cnemial crest, $\mathbf{u}_{1}$ has an anteroproximal inclination (Fig. 31A,B), which is much the same in the lateral cnemial crest, although a variable lateral component may also be present (Fig. 31C,D). In the thinnest parts of the crests where fabric was unable to be quantified, as well as in the cnemial crests of smaller bird tibiae, 3-D visualization of the CT scan data demonstrates that the individual trabeculae tend to maintain this general orientation, essentially following the anterior margins of the crests (Fig. 31E,F). Under the medial articular condyle, $\mathbf{u}_{1}$ projects proximally, up and away from the metaphyseal cortex and arcs posteriorly towards the articular surface, generally 
1341 remaining subparallel to the sagittal plane (Fig. 31G-K). Immediately under the articular surface,

$1342 \mathbf{u}_{1}$ has a posterior inclination of about $20-30^{\circ}$ to the proximodistal of the bone. A similar pattern 1343 occurs for $\mathbf{u}_{1}$ under the lateral articular condyle, although there is also a strong lateral component 1344 to the inclination; sometimes the amount of lateral inclination exceeds the amount of posterior 1345 inclination (Fig. 31L-P). In the central part of the metaphysis, there is sometimes a double1346 arcuate pattern in $\mathbf{u}_{1}$ parallel to the sagittal plane; one 'tract' arcs from the posterior metaphysis to 1347 the cnemial crests, the other arcs from the anterior aspect towards the articular condyles (Fig. 1348 31Q). Notably, this pattern was not observed in all specimens examined, not even in all 1349 specimens of the same species. Furthermore, the orientation of the secondary fabric direction $\mathbf{u}_{2}$ is not constrained to being subparallel to the plane of the arcing, as is the case in the human proximal femur. Progressing distally through the tibial metaphysis and into the diaphysis brings about increased obliquity and disorganization to the orientation of $\mathbf{u}_{1}$ (Fig. 31R). Fabric orientation could not be extensively quantified in the tibiotarsus of many of the smaller bird bones (or even at all in the smallest ones). Nevertheless, 3-D visualization of the trabeculae themselves reveals that, in the regions of the cnemial crests and articular condyles, they tend to be oriented in the same general direction as $\mathbf{u}_{1}$ in the larger bird specimens (Fig. 31E,F,K,P). In the smallest species (e.g., Coturnix chinensis), there are hardly any trabeculae at all in the entire proximal end, with the medullary cavity extending almost to the proximal cortical surface. Additionally, little room for trabeculae exists in the cnemial crests between the two opposing cortices: the crests are either devoid of trabeculae, or there are very small trabeculae acting as spacers in a 'sandwich structure' (Currey 2002).

In the extant sprawling reptiles examined, $\mathbf{u}_{1}$ is generally oriented proximodistally throughout the entire proximal tibia, although it fans out proximally, away from the middle of the bone towards the articular surfaces (Fig. 32). There does not appear to be any appreciable increase in the degree of obliquity and disorganization of $\mathbf{u}_{1}$ in the more distal parts of the metaphysis, as observed in

The proximal tibiae of both Allosaurus and the tyrannosaurids again show similar patterns to each other, as with the femur. Under the medial condyle, strongly developed tracts of cancellous bone are parallel to the sagittal plane and gently inclined posteriorly relative to the proximodistal 
1372 axis (by $5-10^{\circ}$ ); they also have a gentle lateral inclination as well (Fig. 33A,B). This continues

1373 into the region of the lateral condyle, but here the tracts assume a more marked lateral inclination, 1374 similar to the pattern described above for $\mathbf{u}_{1}$ in the birds (Fig. 33B,C). In the cnemial crest, the 1375 dominant direction of cancellous bone is largely parallel to the anterior margin of the crest, and is 1376 oriented proximoanterolaterally, again similar to the pattern described above for $\mathbf{u}_{1}$ in the birds. 1377 In axial cross-section, the cancellous bone actually forms concentric, proximodistally oriented 1378 'sheets' that are parallel to the external surface of the cnemial crest (Fig. 33D-J). Further 1379 posteriorly, towards the base of the cnemial crest, these sheet-like tracts become progressively 1380 more posteriorly inclined, directed toward the metaphysis. In the central metaphyseal region, a

1381

1382

1383

1384

1385

1386

1387

1388

1389

1390

1391

1392

1393

1394

1395

1396

1397

1398

1399

1400

1401 double-arcuate pattern is present, which roughly parallels the sagittal plane, where one set of tracts arcs up from the posterior periphery towards the cnemial crest (Fig. 33D-J, purple and turqoise), and the other set of curved sheets arcs up from the anterior periphery towards the articular condyles (Fig. 33D-J, green and maroon). Furthermore, 3-D visualization indicates that these tracts are curved, more or less concentric with the bone margins. Progressing proximally, the anterior and posterior sets of tracts gradually change inclination to merge with the tracts in the regions of the cnemial crest and articular condyles, respectively. The tyrannosaurids provide a few further details on cancellous bone in the proximal tibia, owing to better contrast in their CT scans compared to those of Allosaurus. Firstly, in the (incipient) lateral cnemial crest, cancellous bone is oriented parallel to the margin of the adjacent part of the main (anterior) cnemial crest (Fig. 33D-J, yellow) Secondly, in the fibular crest the cancellous bone forms a double-arcuate pattern parallel to the axis of the crest (Fig. 33D-J, red and orange); these arcs intersect proximally, and proximally they also curve inwards towards the diaphysis. Thirdly, there is no indication in the distal metaphysis that cancellous bone is anything but oriented parallel to the long axis of the bone.

In the single proximal ornithomimid tibia that was studied (TMP 93.066.0002), the region of the lateral condyle was characterized by a tract of cancellous bone with a slight posterolateral inclination, superimposed on an otherwise proximodistal orientation. This is comparable to the pattern in Allosaurus and the tyrannosaurs. 
1402 The proximal tibia of Troodontidae sp. largely shows the same general patterns for $\mathbf{u}_{1}$ as

1403 described in the birds. Under the medial and lateral condyles, $\mathbf{u}_{1}$ exhibits a gentle posterior 1404 inclination superimposed on an otherwise proximodistal alignment (Fig. 34A-D). However, only 1405 a slight lateral inclination occurs under the lateral condyle, in contrast to the often marked lateral 1406 inclination observed in birds, as well as Allosaurus and the tyrannosaurids. In the cnemial crest, $1407 \mathbf{u}_{1}$ is again oriented largely parallel to the anterior margin of the crest, inclined proximoanteriorly 1408 (Fig. 34E,F). This pattern is also present in the cnemial crest of Saurornitholestes (Fig. 34G). 1409 Throughout the metaphysis of Troodontidae sp., the orientation of $\mathbf{u}_{1}$ is largely proximodistal; a weakly developed double-arcuate pattern, parallel to the sagittal plane, is present in one specimen examined, but not the other (Fig. 34H). This is similar to the large birds studied, where only some specimens exhibited a comparable double-arcuate pattern.

\section{I.4.2.3 Distal tibia or tibiotarsus}

1416

1417

In the human distal tibia, $\mathbf{u}_{1}$ is largely proximodistally oriented throughout the entire bone.

However, throughout most of the metaphysis, $\mathbf{u}_{1}$ has a slight $\left(10^{\circ}\right.$ or so) inclination relative to the proximodistal axis towards the centreline of the bone, forming a conical pattern with the cone's apex pointing distally (Fig. $35 \mathrm{~A}, \mathrm{~B}$ ). The orientation of $\mathbf{u}_{2}$ is generally parallel to the axial plane; it has no orientation preference except in the periphery where it is generally subparallel to the local bone surface, forming a roughly concentric pattern (Fig. 35C).

The distal tibiotarsus of birds shows a characteristic pattern regardless of size (Fig. 36). By and large, $\mathbf{u}_{1}$ is oriented proximodistally and parallel to the sagittal plane throughout the whole distal end of the bone. In large birds for which substantial quantification of fabric was possible, $\mathbf{u}_{2}$ is oriented more or less anteroposteriorly throughout most of the tibiotarsus, in stark contrast to the human pattern, although it can become more parallel to the bone margin towards the periphery (Fig. 36A-E). Within the condyles, $\mathbf{u}_{1}$ and $\mathbf{u}_{2}$ can also become 'rotated' within the sagittal plane to a variable degree. This distinctive pattern reflects the highly anisotropic and plate-like nature of the trabeculae (parallel to the sagittal plane) in this region of the bone, as evident in 3-D visualizations (Fig. 36F-H). In many of the smaller bird species, there was too little cancellous 
1433 bone (too few trabeculae spaced too far apart) to permit an extensive quantitative fabric analysis, 1434 or any analysis at all in the smallest specimens. Nonetheless, 3-D visualization clearly shows that

1435

1436

1437

1438

1439

1440

1441

1442

1443

1444

1445

1446

1447

1448

1449

1450

1451

1452

1453

1454

1455

1456

1457

1458

1459

1460

1461

1462

most of the distal tibiotarsus in these species is dominated by relatively large, usually plate-like

trabeculae that are oriented more or less parallel to the sagittal plane, qualitatively similar to the

architecture observed in larger specimens (Fig. 36F-H). For those species in which fabric

analysis was possible to some degree, the general pattern in $\mathbf{u}_{1}$ and $\mathbf{u}_{2}$ observed for larger birds

was also observed here.

In the distal tibia of extant sprawling reptiles, $\mathbf{u}_{1}$ is predominantly oriented proximodistally, although in the varanids it also takes on a posterior inclination throughout much of the metaphysis (Fig. 37A-C). Distally, $\mathbf{u}_{1}$ tends to fan out towards the articular surface, much in the fashion as described for the proximal tibia. Similar to the human pattern, but unlike the birds, $\mathbf{u}_{2}$ is subparallel to the axial plane and largely concentric with the margins of the bone (Fig. 37D).

The distal tibia of both Allosaurus and the tyrannosaurids examined presents an intriguing cancellous bone architecture (Fig. 38A-G). In the axial plane, it is manifest as a two sets of double-arcuate patterns that are largely parallel to the margins of the bone (Fig. 38D, inset), yet in both the sagittal and coronal planes it is manifest as a more typical double-arcuate pattern, with the arcs from opposing sides of the bone intersecting distally. In three dimensions, this produces a set of 'Gothic arches' (Garden 1961). More distally, these arches progressively open up and become more distally directed, and the sheet-like tracts of cancellous bone become somewhat more anteroposteriorly oriented. They also start to fan away from the centreline of the bone, such that in the distal extremity of the tibia they are oriented medially on the medial side of the bone and are oriented laterally on the lateral side of the bone. Despite this opening up of the arches, a concentric pattern in the axial plane is somewhat retained. Within the astragalus and calcaneum, cancellous bone is only present in considerable quantities in the regions of the articular condyles. Here, the architecture is relatively simple, being strongly aligned in both the anteroposterior and proximodistal directions, paralleling the pattern for $\mathbf{u}_{1}$ and $\mathbf{u}_{2}$ in birds (Fig. 38H-J). This pattern also occurs in the fused astragalocalcaneum of Ceratosaurus nasicornis (UMNH VP 5278). Progressing distally, it also fans out and away slightly from the centreline. Unlike in the 
1463 distalmost tibia, there is no indication of a concentric pattern of cancellous bone in the axial 1464 plane.

1465

1466

In contrast to the complex architectural patterns observed in more stemward theropods, the

1468

1469

1470

1471

1472

1473

1474

1475

1476

1477

1478

1479

1480

1481

1482

1483

1484

1485

1486

1487

1488

1489

1490

1491

1492

1493 architecture in the distal tibia of Troodontidae sp. and Saurornitholestes is relatively simple (Fig. 39). The orientation of $\mathbf{u}_{1}$ throughout the distal tibia is largely subparallel to the proximodistal axis, and $\mathbf{u}_{2}$ is largely contained in the axial plane and aligned anteroposteriorly (except around the periphery), much like birds. Furthermore, 3-D visualization reveals that the cancellous bone architecture is dominated by plate-like trabeculae that are oriented more or less parallel to the sagittal plane, also like birds (Fig. 39C). Within the astragalus and calcaneum of Troodontidae sp., $\mathbf{u}_{1}$ and $\mathbf{u}_{2}$ become somewhat more disorganized, but importantly they largely remain parallel to sagittal plane. Thus, whilst the architecture of the distal tibia is decidedly different to that in the more plesiomorphic theropods examined, the architecture of the proximal tarsals is quite similar, as it is in birds.

\section{I.4.3 Fibula}

In humans, the primary fabric direction $\mathbf{u}_{1}$ is oriented proximodistally throughout the proximal and distal ends of the fibula (Fig. 40A,B). The same general pattern is also present in the extant sprawling reptiles examined, although $\mathbf{u}_{1}$ often fans out and away from the centerline leading towards the articular surfaces (Fig. 40C-F).

In all bird fibulae for which quantitative architectural analysis was possible, $\mathbf{u}_{1}$ is consistently oriented throughout the whole head, directed posteroproximally from the long-axis of the bone and subparallel to the local bone margin (Fig. 40G-I). In those specimens for which quantitative analysis was not possible, 3-D visualization revealed that the trabeculae themselves followed a similar orientation (Fig. 40J,K). There are typically very few trabeculae under the iliofibularis tubercle, even in the large birds, and none in the distal end, which is reduced to a splint of thin cortical bone. 
1494 In the fibular head of Allosaurus and the tyrannosaurids, as well as an indeterminate 1495 ornithomimid (TMP 2006.012.0065), cancellous bone is largely proximodistally orientated. It

1496

1497

1498

1499

1500

1501

1502

1503

1504

1505

1506

1507

1508

1509

1510

1511

1512

1513

1514

1515

1516

1517

1518

1519

1520

1521

1522

1523

1524

gently fans out away from the centreline of the bone in the sagittal plane, paralleling the anterior and posterior margins of the head and leading up towards the articular surface (Fig. 40L,M). In the distal fibula, the dominant architectural direction is largely parallel to the local centerline of the bone, and progressing distally this acquires a gentle lateral inclination (Fig. 40L,M). The proximal fibula of Troodontidae sp. exhibited a pattern in $\mathbf{u}_{1}$ that was comparable to that observed in the other theropods examined (Fig. 40N); the distal end of the Troodontidae sp. fibula that was studied was not preserved.

\section{I.4.4 Diaphyses}

As noted above, the diaphysis of birds and other extant reptiles often contains a significant volume of cancellous bone, in both the femur and tibia. In bird femora, and to a lesser extent the tibia, the most conspicuous feature of this diaphyseal cancellous bone is the abundant trabeculae that are obliquely oriented relative to the long-axis of the bone, typically by $45^{\circ}$ or more (Fig. 41). These trabeculae vary in individual form, displaying a range of rod-like to plate-like morphologies. They also vary in the degree to which they are connected to the adjacent cortex, ranging from being tightly appressed to the cortex (appearing little more than large 'wrinkles' in the endosteal surface), to being well separated from the cortex except at their ends, and arcing across the medullary cavity. When considered across the diaphysis as a whole, these oblique trabeculae tend to form conjugate helices that spiral along the endosteal margin of the cortex, especially in the bones of larger species (Fig. 41A,D,I). Markedly oblique trabeculae are also often present in the diaphysis of the sprawling reptile femora and tibiae examined, although they are not usually as abundant compared to the birds.

The results of the categorical scoring analyses of cancellous bone architecture in bird femora and tibiotarsi are presented in Table 2 and Fig. 42. The intraclass correlation coefficient (ICC) was moderate to good (Koo \& Li 2016) for all three femoral features (0.69-0.85), but was poor (0.46) for the one feature able to be scored for the tibiotarsus. For $n=5$ scorers, this suggests that the 
1525 results of the reliability of the scoring should be viewed tentatively, especially as concerns the 1526 tibiotarsus. In both the femur and tibiotarsus, the extent to which the diaphysis is occupied by 1527 cancellous bone (feature 1) tends to increase in larger bones, although in the tibiotarsus the 1528 increase is only really noticeable for the largest birds (Fig. 42A,B). The average orientation of the 1529 trabeculae relative to the long axis (feature 2) also changes with size in the femur (Fig. 42C), but 1530 not the tibiotarsus (Fig. 42D). In the femur, it increases from approximately $45-50^{\circ}$ in the largest 1531 birds to around $70^{\circ}$ or more in the smallest birds. The average degree of association of trabeculae 1532 with other trabeculae (feature 3) appears to increase in larger femora and tibiotarsi (Fig. 42E,F); 1533 however, the results were not statistically significant. Thus, generally speaking, as bird femora 1534 get smaller, they become occupied by progressively less cancellous bone, the individual 1535 trabeculae of which are fewer in number and more widely spaced, and which have a more 1536 perpendicular orientation to the long axis of the bone. Additionally, as bird tibiotarsi get smaller, 1537 they also become occupied by progressively less cancellous bone, but the arrangement of 1538 individual trabeculae does not appear to change significantly.

1539

Investigation of the human bones in this study revealed that a small amount of cancellous bone occurs along the endosteal surface of much of the tibial diaphysis. Whilst of insufficient quantity for quantitative fabric analysis, it is noteworthy that near the middle of the diaphysis, the cancellous bone architecture is dominated by trabeculae (or endosteal 'wrinkles') that are obliquely oriented, by about $10-20^{\circ}$ to the long-axis of the bone (Fig. 43). Thus, some degree of similarity is present between the diaphyseal cancellous bone of humans and birds.

\section{$\underline{\text { I.5 Discussion }}$}

This study had two primary objectives, first of which was the broad-scale comparative assessment of cancellous bone architecture in the main hindlimb bones of avian and non-avian theropods, as well as in humans and some large extant sprawling reptiles. This comparative assessment focused on the gross architectural features across the whole bone, and used both quantitative and qualitative observations. The second main objective of the study was to draw upon the comparative assessment to identify patterns of similarity and contrast between the 
1556 different groups examined, which may be used to provide insight into bone loading and 1557 locomotor biomechanics.

1558

1559

1560

1561

1562

1563

1564

1565

1566

1567

1568

1569

1570

1571

1572

1573

1574

1575

1576

1577

1578

1579

1580

1581

1582

1583

1584 1585

\section{I.5.1 Overarching patterns across taxa}

Despite great differences in size, and to a lesser degree, phylogenetic heritage, all birds investigated showed largely consistent cancellous bone architecture for a given region of a given bone. The patterns illustrated by birds were often in stark contrast to that exhibited by humans. The extant sprawling reptiles examined (varanid lizards and crocodilians) were also largely consistent in their observed architectural patterns across taxa, and typically showed greater similarity to the architectural patterns of birds than humans.

Among non-avian theropods, there are a number of different patterns of cancellous bone architecture in the femur. In the non-maniraptoriform theropods examined (Masiakasaurus, Allosaurus and tyrannosaurids), the femur showed marked similarity to the architecture observed in humans. In the proximal end, a pronounced double-arcuate pattern occurs in the coronal plane (visible in the tyrannosaurid specimens), and the primary fabric direction of cancellous bone $\left(\mathbf{u}_{1}\right)$ in the femoral head had little anterior inclination, much like humans but unlike birds. In the distal femur, the primary architectural direction in the condyles did not have the often large posterior inclination as observed in birds, but it was often still greater than in humans. Additionally, the primary architectural direction in the condyles did not show the large amount of anteroposterior sweeping as observed in birds. The derived non-avian theropod Troodontidae sp. had a femoral architecture more closely resembling that of birds than most other non-avian theropods examined: no coronal plane double-arcuate pattern was present, $\mathbf{u}_{1}$ in the femoral head had a significant anterior inclination, and in the diaphysis-ward parts of the metaphysis the primary fabric vectors were disorganized and often oblique to the long-axis of the bone. This latter feature, ubiquitous in the femora and tibiae of birds and extant sprawling reptiles, is interpreted to reflect the onset of markedly oblique trabecular spirals in the diaphysis (see Section I.5.4). 
1586 The ornithomimid and caenagnathid femora examined illustrated a distinct and intriguing pattern

1587 in the proximal end that was not observed in any extant groups studied. Most conspicuously, $\mathbf{u}_{1}$ 1588 was oriented predominantly in an anteroposterior direction, with the secondary fabric direction

$1589\left(\mathbf{u}_{2}\right)$ oriented more or less proximodistally. Additionally, $\mathbf{u}_{1}$ tended to exhibit some significant 1590 amount of obliquity and disorganization in the diaphysis-ward parts of the proximal and distal 1591 metaphyses, and in this respect was comparable to the pattern observed in birds and sprawling 1592 reptiles. The femur of the basal therizinosauroid Falcarius shows some similarity to the 1593 ornithomimid and caenagnathid pattern in that the orientation of $\mathbf{u}_{2}$ in the central metaphysis and 1594 head was anteroposteriorly aligned, much like $\mathbf{u}_{1}$ in the ornithomimid femora. This is unlike the 1595 pattern observed in humans, or expected in Allosaurus and the tyrannosaurids based on the 1596 observed architectural patterns. However, the orientation of $\mathbf{u}_{1}$ in the diaphysis-ward parts of the 1597 proximal and distal metaphyses of Falcarius tended to be organized and subparallel to the long1598 axis of the bone, more comparable to humans and more plesiomorphic theropods. Distally, the 1599 femoral condyles of the ornithomimids, caenagnathid and Falcarius all had a gentle posterior 1600 inclination to the direction of $\mathbf{u}_{1}$, with little anteroposterior sweeping of the fabric, more like that 1601 in humans than birds.

1602

1603 The proximal tibia and fibula of the non-avian theropods showed a largely consistent 1604 architectural pattern, although it is acknowledged that these bones were not as extensively sampled as the femur. The architecture of the proximal tibia was quite comparable to that of birds, in both the cnemial crests and underneath the articular regions. A double-arcuate pattern, parallel to the sagittal plane, is present in the proximal end of the tibia of the large non-avian species, but is only occasionally present in Troodontidae sp. and large birds.

Cancellous bone architecture in the proximal tarsals (astragalus and calcaneum) was broadly comparable across all theropods examined, both avian and non-avian, being dominated by a strong anteroposterior-proximodistal alignment. In contrast, two distinctly different architectures were observed in the distal tibia (in non-avian theropods) or tibial component of the tibiotarsus

1614 (in birds). In the large non-maniraptoriform theropods (Allosaurus and tyrannosaurids), the distal 1615 tibia exhibited a complex double set of double-arcuate patterns, parallel to both the sagittal and 1616 coronal planes. In Troodontidae sp., Saurornitholestes and birds, however, the architecture was 
1617 very much a continuation of that observed in the proximal tarsals, with a relatively simple pattern 1618 of anteroposterior-proximodistal alignment of $\mathbf{u}_{1}$ and $\mathbf{u}_{2}$.

1619

1620

1621

Taken together, the various architectural patterns observed in the various bones of the non-avian

1622 theropods show a general correspondence with their phylogenetic relationships (Fig. 44). The more stemward theropods investigated tended to possess architectures that were more broadly comparable, and sometimes strikingly similar, to those in humans. In contrast, the paravians Troodontidae sp. and Saurornitholestes (as far as can be observed) possessed architectures more comparable to those of extant birds. Intriguingly, of all the non-avian theropods examined, Allosaurus and the tyrannosaurids had the greatest degree of posterior inclination in the fabric of the femoral condyles, most like birds (Fig. 44, character B). A further point of interest concerns the ornithomimid and caenagnathid specimens. Their femoral architectural patterns were similar to each other but distinctly different to those in other theropods studied, yet Ornithomimosauria and Oviraptorosauria are not thought to be each other's closest relatives, nor do their lineages branch from successive nodes in theropod phylogeny (Zanno et al. 2009; Fig. 44, characters A, C and F). Thus, if taken at face value, the present set of observations suggest some amount of convergence in cancellous bone architectures, and by inference mechanical loading, between the two groups, or alternatively a reversion to a more plesiomorphic (tyrannosaurid-like) architecture in early therizinosaurs. Neither scenario can be tested until further specimens, of more species and more individuals of each species, are examined.

\section{I.5.2 The importance of holistic analyses}

The results of this study re-affirm the benefit of investigating cancellous bone in a holistic fashion, by considering patterns of architectural variation across whole bones and across multiple bones (Georgiou et al. 2018; Gross et al. 2014; Kivell 2016; Ryan \& Test 2007; Saers et al. 2016; Saparin et al. 2011; Scherf 2008; Skinner et al. 2015; Stephens et al. 2016; Su et al. 2013; Tsegai et al. 2013; Tsegai et al. 2017). Here, the integration of both qualitative and quantitative observations, across several bones, has helped identify many patterns of similarity and contrast between various theropod and non-theropod groups. Few of these patterns would be evident on 
1648 the basis of a single observation alone. Indeed, considering only one or two regions in isolation of 1649 the others may well mislead the investigator into making incorrect interpretations. For example, 1650 the human specimen studied falls within or very near the cloud of data points for birds on 1651 stereographic plots of the mean orientation of $\mathbf{u}_{1}$, in both the femoral head (Fig. 22A) and medial 1652 femoral condyle (Fig. 29A). In isolation of all other observations, this would lead to the false

1653

1654

1655

1656

1657

1658

1659

1660

1661

1662

1663

1664

1665

1666

1667

1668

1669

1670

1671

1672

1673

1674

1675

1676

1677

1678

conclusion that humans have a posture and locomotor biomechanics not too different from that of extant birds. Other observations, such as the double-arcuate pattern of $\mathbf{u}_{1}$ and $\mathbf{u}_{2}$ in the coronal plane of the proximal femur (present in humans but absent in birds), marked anteroposterior sweeping of $\mathbf{u}_{1}$ in the femoral condyles (absent in humans but present in birds) and often abundant, markedly oblique diaphyseal trabeculae (absent in humans but present in birds) point to a very distinct difference in locomotor biomechanics between humans and birds, a fact borne out in many experimental studies (Bishop et al. 2018, and references cited therein; Gatesy \& Biewener 1991). Only by considering all available cancellous bone architecture, across whole bones and across multiple bones, can the similarities and differences between species be truly appreciated, and only though this may a robust set of biomechanical inferences be developed. Moreover, in the case of extinct, non-avian theropods, future study of more species and more bones can add to the dataset produced here, further improving biomechanical inferences (see also below).

\section{I.5.3 Biomechanical implications}

As noted above, birds and humans demonstrate many distinct differences in cancellous bone architecture throughout their hindlimb bones. This was to be expected, since they exhibit very different bipedal locomotor biomechanics. Here, an attempt is made to correlate the salient differences in architectural patterns and biomechanics, and use these inferences to provide insight into the hindlimb locomotor biomechanics of the non-avian theropods studied. 
The mean orientation of $\mathbf{u}_{1}$ in the femoral head of the human specimen had only a very small anterior inclination, whilst in birds the inclination was on the whole far more pronounced (Fig. 22A). This is interpreted to reflect the stark difference in femoral posture between humans and birds, with a large degree of flexion at the hip joint in birds leading to the hip joint reaction force being more anteriorly directed relative to the femur (Fig. 45). It cannot be discounted at present that the differences in architecture may also reflect differences in trunk posture between humans (orthograde) and birds (pronograde), although how trunk posture may influence hip joint loading remains unexplored. Most non-avian theropods examined had a minor anterior component to the mean orientation of $\mathbf{u}_{1}$, as in the human, suggesting that these species also held their femur in a similar, subvertical orientation. However, the mean orientation of $\mathbf{u}_{1}$ in Troodontidae sp. lay between that of birds and the other non-avian theropods, suggesting that its femoral orientation was intermediate between the subvertical posture of humans and the subhorizontal posture of birds.

\section{I.5.3.2 Medial femoral condyle}

1698

Much as in the femoral head, the mean orientation of $\mathbf{u}_{1}$ in the medial femoral condyle is telling of postural differences between humans and birds, paralleling the results of previous experimental studies (Polk et al. 2008; Pontzer et al. 2006). In the human, it had only a small posterior inclination, whereas in birds the posterior inclination was generally substantial (Fig. 29A), which is inferred to reflect the greater degree of habitual knee flexion in birds (Fig. 45). This is supported by the results for the extant sprawling reptiles studied, which also exhibited a marked posterior inclination to $\mathbf{u}_{1}$ (although this was not strictly quantified), reflecting a marked level of habitual knee flexion during locomotion (Blob \& Biewener 2001; Clemente et al. 2013; Gatesy 1991a). It was also demonstrated that the mean orientation of $\mathbf{u}_{1}$ in smaller birds tends to be more posteriorly inclined compared to larger birds (Fig. 29B). This reflects the fact that smaller birds tend to have a larger degree of postural crouch (Bishop et al. 2018; Gatesy \& 
1710 Biewener 1991), which can be brought about by greater flexion of the knee joint. The non-avian

1711 theropods examined showed a variable amount of posterior inclination in the mean orientation of

$1712 \mathbf{u}_{1}$, although on the whole it was usually less compared to birds (less than $20^{\circ}$; Fig. 29A),

1713 suggesting a level of habitual knee flexion intermediate between that of humans and birds. No

1714 phylogenetic pattern was apparent, and curiously the Allosaurus-tyrannosaurid architecture had

1715 the greatest posterior inclination of all.

1716

1717 A further point of difference between birds and the other groups investigated here is the degree to 1718 which the orientation of $\mathbf{u}_{1}$ swept throughout the condyles in the anteroposterior plane. In birds, 1719 this sweeping often exceeded $100^{\circ}$, yet it was less than $40^{\circ}$ in all other groups (although this was 1720 not strictly quantified). This may reflect the greater degree of habitual knee flexion birds, but it 1721 may also correlate to the greater range of knee flexion-extension employed by birds during the 1722 stride cycle compared to other groups (Andrada et al. 2013; Blob \& Biewener 2001; Clemente et 1723 al. 2013; Cracraft 1971; Gatesy 1991a; Gatesy 1999; Kambic et al. 2014; Kambic et al. 2015; 1724 Reilly 2000; Rubenson et al. 2007; Stoessel \& Fischer 2012; Winter 2009). It should be noted 1725 that, owing to the fact that $\mathbf{u}_{1}$ swept throughout the condyles, the mean direction results reported 1726 for the medial condyle above need to be viewed with some caution. This is because significant 1727 sweeping in the anterior part of the condyles, particularly in larger species, could influence the 1728 calculated mean direction.

I.5.3.3 Proximal and distal femur

1732

1733

The pronounced double-arcuate architecture of cancellous bone in the coronal plane of the human 1734 proximal femur has been widely recognized for nearly two centuries (Ward 1838) and much discussion has focused upon the mechanical significance of this for almost as long (see reviews by Cowin 2001; Skedros \& Baucom 2007). Despite the various interpretations that have been proposed over time, the inescapable fact remains that this bears strong resemblance to the principal stress trajectories that would be engendered under mediolateral bending, via a load applied to the head of the femur (the 'trajectorial theory'; see Introduction and Fig. 4). Such a loading regime, with compression dominating medially and tension laterally, is supported by in 
1741 vivo strain gauge data (Aamodt et al. 1997). Moreover, the 'primary compressive group' that runs

1742 from the base of the femoral neck up to the head has been widely considered as reflecting

1743 transmission of the hip joint reaction force, away from the hip and down toward the rest of the

1744 femur (Skedros \& Baucom 2007). The presence of a strikingly similar double-arcuate pattern,

1745 also parallel to the coronal plane, in the proximal femur of the tyrannosaurids suggests that very

1746 much the same loading environment as occurs in humans also occurred in these species.

1747 Mediolateral bending of the femur is also suggested by another double-arcuate pattern, parallel to

1748 the coronal plane, in the distal femoral metaphysis.

1749

1750 An additional point of note is that the 'primary compressive group' in Allosaurus and the 1751 tyrannosaurids is directed towards the apex of the femoral head. By analogy with the proximal 1752 femur of humans, this suggests that the hip joint reaction force was principally applied there, 1753 implying that the articulation with the acetabulum was centred about the apex of the femoral 1754 head. This interpretation differs from previous suggestions, that the articulation was more lateral 1755 and involved at least part of the trochanteric region (e.g., Hotton 1980; Hutchinson \& Allen 1756 2009). The exact manner in which non-avian theropod hips articulated is therefore worthy of 1757 further study. Indeed, how the femur and acetabulum contacted each other may have varied both 1758 within and between various behaviours (e.g., differing degrees of hip abduction). These dynamic 1759 articulations could have possibly varied with different osteological morphologies (e.g., different 1760 degrees of inclination of the femoral neck) or soft tissue arrangements (Tsai \& Holliday 2015;

1761 Tsai et al. 2018), and probably changed appreciably on the line from basal theropods to extant 1762 birds.

1763

1764

1765

$\underline{\text { I.5.3.4 Lesser trochanter }}$

1766

1767 A second double-arcuate architecture in the lesser trochanter of the femur of the tyrannosaurids, 1768 also parallel to the coronal plane, further suggests that the trochanter also was loaded 1769 predominantly in mediolateral bending. This could conceivably occur via the medial pull (or 1770 medial component thereof) of the muscle(s) that inserted there, such as the iliotrochantericus 
1771 caudalis (Hutchinson 2001a). In such a situation it would be predicted that the medial arcade

1772 would be loaded in compression, and the lateral arcade in tension.

1773

1774

1775 I.5.3.5 Proximal tibia

1776

1777 The orientation of $\mathbf{u}_{1}$ in the proximal tibia of the human is largely proximodistally oriented

1778 throughout the entire end, whereas it shows considerable variation throughout the bone in birds.

1779 Under the articular condyles, birds exhibit a more marked posterior inclination compared to that

1780 in the human (up to $30^{\circ}$, versus about $10^{\circ}$ ), as well as a strong lateral component under the lateral

1781 condyles, which does not occur in humans. Anteriorly, $\mathbf{u}_{1}$ can take on a distinct anterior

1782 inclination as it parallels the leading margin of the cnemial crests, which are absent in humans.

1783 Within the metaphysis, a double-arcuate pattern in $\mathbf{u}_{1}$, parallel to the sagittal plane, may also

1784 occur in the proximal tibia of birds, which is also absent in humans. In these respects, the

1785 proximal tibia of all the non-avian theropods studied is more similar to that of birds. This

1786 similarity in cancellous bone architecture is undoubtedly due in part to the greater similarity in

1787 morphologies (e.g., prominent cnemial crest) and nature of the knee articulation (with the fibula

1788 being involved laterally) between the two groups. However, it does suggest that anteroposterior

1789 bending may be a more significant loading regime in the theropod tibia than the human tibia.

$\underline{\text { I.5.3.6 Distal tibia or tibiotarsus }}$

1793

1794 The distal tibia of Allosaurus and the tyrannosaurids has two well-developed sets of double-

1795 arcuate patterns, one parallel to the sagittal plane, the other parallel to the coronal plane. By

1796

1797 analogy with the proximal femur, this suggests that both anteroposterior bending and

1798 mediolateral bending were important loading regimes in this part of the bone. These two different loading regimes may possibly have been engendered during different behaviours, or at different instances during the one behaviour, such as different points throughout the stride cycle. Conspicuously, these complex patterns do not continue into the astragalus and calcaneum. Equally conspicuous is the different cancellous bone architecture in the distal tibia of 
1802 Troodontidae sp., Saurornitholestes and birds, which is continuous with the architecture in the 1803 astragalus and calcaneum (in Troodontidae sp. and birds at least). Not only does this suggest 1804 tighter mechanical unity between the three bones in life in the case of Troodontidae sp. (indeed, 1805 the astragalus and calcaneum fuse in adults), but it also suggests that the distal tibiotarsus of 1806 Troodontidae sp. and Saurornitholestes experienced a different set of loading regimes compared 1807 to Allosaurus and the tyrannosaurids, but similar to that of extant birds.

1808

1809

1810

\section{I.5.4 Oblique trabeculae in the diaphyses}

1811

1812

One of the more interesting results of this study was the observation of markedly oblique trabeculae in the diaphysis of the femur and tibia of birds and extant sprawling reptiles. Aside from some pterosaur wing bones (Wellnhofer 1991), the authors are not aware of this feature being reported previously for any other tetrapod group. Interestingly, however, it also appears to be present in the proximal humeral diaphysis of orangutans (Pongo pygmaeus), judging from a figure published by Scherf et al. (2013, figure 1a). In the present study, the observed oblique trabeculae tended to form helices that spiralled along the endosteal margin, especially in the bones of larger bird species. This feature is interpreted to be responsible for the progressive increase in obliquity and disorganization of the orientation of $\mathbf{u}_{1}$ in the diaphysis-ward part of the metaphysis in birds and sprawling reptiles. Essentially, the more ordered architecture of the main part of the metaphysis gradually breaks down and transitions to a sparser architecture of oblique trabeculae in the diaphysis.

Application of the trajectorial theory to the oblique trabeculae of the diaphysis of bird and reptile femora and tibia would suggest that these bones are loaded predominantly in torsion, or at the very least experience a significant amount of torsion during daily use. This is because for a cylinder under pure torsion, both maximum (tensile) and minimum (compressive) principal stresses are parallel to the margin and oriented at $45^{\circ}$ to it, forming conjugate spirals (Beer et al. 2012; Carrano 1998). In vivo strain gauge studies fully support this interpretation: the femora and tibiae of both birds and reptiles are loaded predominantly by torsion during locomotion 
1833 Biewener 1999; Main \& Biewener 2007; Verner et al. 2016). Oblique trabeculae were also 1834 observed to occur in the diaphysis of the human tibia, although not as strongly oblique to the 1835 bone's long-axis compared to birds and reptiles (about 10-20 ). This also concurs with in vivo 1836 data showing that a considerable torsional component to bone loading occurs during part of the 1837 stance phase of locomotion (Lanyon et al. 1975; Yang et al. 2014). The increase in obliquity and 1838 disorganization of $\mathbf{u}_{1}$ observed in the diaphysis-ward parts of the femoral metaphyses of 1839 Troodontidae sp., and to a lesser extent the ornithomimids and caenagnathid, therefore suggests 1840 that torsion was a more important (but not necessarily predominant) loading regime in the femur 1841 of these species. By contrast, the lack of any noticeable obliquity in $\mathbf{u}_{1}$ in the femora of the other 1842 non-avian theropods studied implies that torsion was minimally important. This too concurs with 1843 observations of the human femur, whereby $\mathbf{u}_{1}$ is subparallel to the long-axis of the bone in the 1844 diaphysis-ward parts of the metaphyses.

1845

Whilst spiralling trabeculae in the femoral and tibial diaphyses of large birds is consistent with predictions of the trajectorial theory, the agreement breaks down in the bones of smaller birds. Specifically, in smaller birds the trabeculae tended to acquire an increasingly oblique orientation relative to the long-axis, approaching $70^{\circ}$ or more; indeed, in some specimens, there were individual trabeculae that were almost orthogonal to the long-axis. Presumably, these smaller bird bones are also loaded predominantly in torsion, as are the bones of their larger relatives, on account of there being no evidence to the contrary, by way of anatomical, kinematic or kinetic observations. It would therefore be expected that principal stresses would still be approaching $45^{\circ}$ to the long-axis of the bones. The lack of congruence between trabecular orientation and predictions of the trajectorial theory warrants explanation.

One possible explanation for the observed architectural patterns in smaller bird bones is that these bones are probably more liable to undergo failure through torsion-induced buckling, compared to the bones of larger species. In torsional loading, the critical shear stress needed to initiate buckling in a thin-walled cylindrical tube is given by

$$
\tau_{\text {crit }}=\frac{k \pi^{2} D}{l^{2} t},
$$


1862 where $k$ is a constant depending on the comprising material, $l$ is the length and $t$ is the thickness 1863 of the cylinder wall (Batdorf 1947; Batdorf et al. 1947; Donnell 1933; Weingarten et al. 1968). D 1864 is the flexural stiffness per unit length along the circumference, given as

1865

$$
D=\frac{E t^{3}}{12\left(1-v^{2}\right)},
$$

1866

1867

1868

1869

1870

1871

1872

1873

1874

where $E$ is Young's modulus and $v$ is Poisson's ratio for the material concerned. Moreover, the stress in a thin-walled tube loaded in torsion is related to the applied torque $T$ as

$$
\tau=\frac{T}{2 t A}
$$

where $A$ is the area of the cross-section (Beer et al. 2012); for a circular geometry, this means that

$$
\tau=\frac{2 T}{\pi d^{2} t}
$$

where $d$ is the diameter. Therefore, the critical torque required to initiate buckling may be expressed as

$$
T_{\text {crit }}=\frac{K \pi^{3} d^{2} t^{3}}{2 l^{2}},
$$

where $K$ is a constant reflecting the material comprising the tube. A tube with a higher value of $T_{\text {crit }}$ requires a higher applied torque for buckling to initiate, and hence a higher value of $T_{\text {crit }}$ implies a lower propensity to buckle at a given load. Thus, the propensity for the tube to undergo torsion-induced buckling is proportional to the square of its length and inversely proportional to the square of its diameter. Previous studies of bird allometry have demonstrated that at smaller size, their hindlimb bones become progressively more slender (Alexander 1983; Brassey et al. 2013; Carrano 1998; Doube et al. 2012; Gatesy 1991b; Olmos et al. 1996). Conversely, for a given size-normalized cross-sectional geometry, bones that are smaller in absolute terms will be longer in relative terms. The femora and tibiotarsi of smaller bird species may therefore be more prone to torsion-induced buckling. One way by which to mitigate buckling in a thin-walled tube is to support the tube walls against excessive transverse deflection, through the addition of structural stiffeners inside the tube (Chitale \& Gupta 2011). It is therefore hypothesized that the high-angle trabeculae in the femora (and less frequently, the tibiotarsus) of small birds are present mainly to provide cross-bracing support. By stiffening the diaphysis, they help prevent the 
1888 dimensions of the bone changing too much to the point that buckling is initiated, which could 1889 lead to catastrophic failure at the whole-bone level.

1890

1891

1892

\section{I.5.5 Future work}

1893

1894

1895

It is worth reiterating the main limitations of the present study, and noting that these may be

1896 addressed in future investigations. The foremost limitation concerns that of sampling. In investigating phenomena not studied previously, the work presented here was very much exploratory. Logistical constraints restricted the number of species that were studied, as well as the number of replicates for each species and each bone. It was therefore not possible in many instances to provide a more precise, quantitative assessment of architectural variation, in terms of inter- or intraspecific variability, or how architectural patterns may relate to finer-scale differences in anatomy, behaviour or habitat. However, the data reported in Fig. 22B does suggest, at least qualitatively, that the potential for intraspecific variation in extant birds may be significant, and that intraspecific variation is therefore worthy of future scrutiny. In addition, although large specimens were sought for the extinct, non-avian theropods, this nevertheless could not fully control for possible ontogenetic influences, as recognizing skeletally mature adults in the fossil record can be problematic (Hone et al. 2016). Furthermore, a number of major non-avian theropod groups were not investigated, such as basalmost tetanurans (e.g., megalosaurs), coelophysoids or alvarezsaurids. Increased sampling of non-avian species, as well as the number of individuals or bones for each species, is therefore an important objective for future studies, and will likely lead to greater refinement of the general patterns identified here, as well as subsequent biomechanical interpretations. Likewise, it will also be important to increase sampling of avian species, including those outside of the crown group, although as many fossils of stem-group birds are taphonomically flattened, this will admittedly be difficult. In addition, it may be worthwhile expanding the scope of study to include other bones as well; for instance, investigating cancellous bone architectural patterns in the ilium may provide further insight on hip joint loading mechanics and posture (cf. sections I.5.3.1 and I.5.3.3 above). 
1918 It would also be very worthwhile investigating potential effects of body size in non-avian 1919 theropods, by conducting denser sampling within lineages that display marked variation in body

1920

1921

1922

1923

1924

1925

1926

1927

1928

1929

1930

1931

1932

1933

1934

1935

1936

1937

1938

1939

1940

1941

1942

1943

1944

1945

1946

1947

size (e.g., dromaeosaurids, tyrannosauroids). This can not only provide further illumination on

the range of postures and locomotor biomechanics used by non-avian theropods, but may also help disentangle the relative influence of body size from other factors, such as phylogeny, musculoskeletal anatomy or the location of the whole-body centre of mass. For instance, the greatest amount of posterior inclination of the mean orientation of $\mathbf{u}_{1}$ in the medial femoral condyle was observed in Allosaurus and the tyrannosaurids, which were also the largest species studied. This observation is counter to what would be predicted from extant birds (Fig. 29B), and so may reflect other factors. Alternatively, this observation may indeed reflect their very large size, where at such large body size other factors become relevant, factors that are not important at smaller sizes.

A second limitation of this study concerns the fact that focus was directed primarily towards the directionality of cancellous bone fabric. Whilst architectural directionality is an important indicator of loading mechanics (see Section I.2.3 above), studies of extant species have demonstrated that other architectural parameters can also be useful, such as bone volume fraction, trabecular thickness and trabecular spacing. Future investigation of these parameters may therefore yield further insights. A serious obstacle to this line of enquiry, however, is being able to achieve sufficient CT scan contrast and resolution with fossil specimens, which currently is only possible for smaller specimens. Additionally, such study also requires excellent preservation of an entire fossil specimen.

One final methodological limitation worth noting here is that the quantitative architectural analyses performed were not able to be used in as extensive a fashion - if at all - in very small bird bones. This was because these analyses rested on the continuum assumption, which breaks down at small spatial scales. Essentially, there were too few trabeculae present to permit rigorous quantitative analysis. A goal for future studies may hence be to explore alternative ways of quantitatively characterising cancellous bone architecture in very small bones. One possible avenue is to use micro-finite element modelling (Ryan \& van Rietbergen 2005; van Rietbergen et 
1948 al. 2003; van Rietbergen et al. 1999) to examine the principal material directions of the 1949 cancellous bone structure directly, although this can be computationally very expensive. 1950

A further avenue for future work concerns the oblique or spiralling patterns of trabeculae observed in the femoral and tibial diaphyses of many birds, as well as other species. These patterns have hitherto never been reported outside of pterosaurs (Wellnhofer 1991), and so it would be interesting to investigate how widely distributed they actually are among various tetrapod groups. It would also be worthwhile exploring more quantitative and objective methods of characterizing diaphyseal cancellous bone architectures; a simple categorical scoring approach was used in the present study, which if employed in further studies should employ greater numbers of scorers than that used here (five). Given the mechanical significance hypothesized above for the spiralling patterns observed in many bones examined in the present study, it would also be interesting to investigate whether their presence correlates with certain aspects of locomotor biomechanics.

\section{$\underline{\text { I.6 Conclusion }}$}

This study has used new approaches for analysing and quantifying how the 3-D architecture of cancellous bone varies throughout a limb bone, as well as new ways of comparing this architecture between species. In doing so, it has produced a broad survey of the major architectural features present in the main hindlimb bones of a variety of extinct, non-avian theropod species, as well as a variety of extant, ground-dwelling birds.

Qualitative and quantitative comparisons between non-avian theropods, birds, sprawling reptiles and humans have identified several patterns of similarity and contrast between these groups. Many of the observed patterns can be mechanistically linked to various aspects of locomotor biomechanics in the extant species, such as the degree of hip or knee flexion. This has in turn provided insight into locomotor biomechanics in non-avian theropods. Although explicit quantitative comparisons were conducted only for two regions of the femur in the present study, the approach used here can be expanded to the analysis of other regions of this and other bones in 
1979 the future. Not only will this enable a more rigorous characterization of cancellous bone

1980 architectural variation in the various species, but it may also provide further bearing on

1981 interpretations of locomotor biomechanics, especially with increased sample sizes.

1982

1983

Cancellous bone architecture in the hindlimb bones of birds is quite consistent across the species 1984 studied. When variations were apparent, they could be related to differences in size, or the 1985 presence or absence of pneumatization. Although variation due to phylogeny was not explicitly tested for in this study, no evidence for this was apparent. For instance, comparably-sized kiwis and chickens exhibited similar architectural patterns, as did comparably-sized tinamous and quail.

Broadly speaking, the cancellous bone architecture in more plesiomorphic theropods (ceratosaurs, Allosaurus and tyrannosaurids) is comparable to that in humans in many respects, but is often distinctly different from that observed in birds. The architectural patterns observed in Troodontidae sp. (and Saurornitholestes, where it was possible to assess) are typically intermediate between those of humans and birds. However, some features, such as the architecture of the distal tibiotarsus, are essentially identical to that of birds. Ornithomimid and caenagnathid femora both show a fairly distinct architectural pattern, different from all other groups studied. In particular, the primary fabric direction in the femoral head is largely anteroposteriorly aligned, and the fabric exhbits an axially radiating pattern in the ditstal femur.

Cancellous bone architecture in the hindlimb bones of non-avian theropods clearly varies architectural features in the more plesiomorphic theropods studied suggest a manner of locomotion not too dissimilar from humans, with a subvertical femoral posture and mediolateral bending being the dominant loading regime in the femur. In contrast, Troodontidae sp. is inferred to have had locomotor biomechanics intermediate between those of the more plesiomorphic theropods and extant birds, befitting its phylogenetic position.

2006

A particularly interesting architectural feature observed in the present study is the abundance of markedly oblique trabeculae in the diaphyses of the femur and tibia of birds, and to a lesser 2009 extent, extant crocodilians and lizards. In the bones of large species, this produces spiralling 
2010 patterns along the endosteal surface of the diaphysis. It is hypothesized that this feature reflects a 2011 prominence of torsional loading in these bones during normal use. If this is correct, the presence 2012 of oblique or spiralling trabeculae can be used as an indicator of high-torsion limb bone loading 2013 in future studies of other extinct vertebrate species.

2014

2015

2016

\section{I.7 Acknowledgements}

2017

2018

The staff of the Geosciences Program of the Queensland Museum is thanked for the provision of 2019 workspace and access to literature: A. Rozefelds, K. Spring, R. Lawrence, P. Tierney, J. Wilkinson and D. Lewis. A very special thanks is extended to the staff and associated colleagues of the institutions that provided access to the material studied here: D. Henderson, B. Strilisky, G. Housego, R. Russel, T. Courtenay, B. Sanchez and F. Therrien (Royal Tyrell Museum of Palaeontology, Drumheller); R. Irmis, C. Levitt-Bussian, C. Webb and P. Policelli (Natural History Museum of Utah, Salt Lake City); J. Horner, J. Scannella, D. Varricchio, D. Strosnider, C. Woodruff, D. Fowler and T. Carr (Museum of the Rockies, Bozeman); K. Spring, H. Janetzki, A. Amey, P. Couper and S. Van Dyck (Queensland Museum, Brisbane); K. Roberts (Museum Victoria, Melbourne); R. Sadlier (Australian Museum, Sydney); and M. Forwood (Griffith University, Gold Coast). Many of the above people also provided helpful discussion on various aspects of theropod biology, and also helped transport specimens for CT scanning. The efforts of those who facilitated or performed the scanning itself are also much appreciated: S. Purdy and D. Wetter (Canada Diagnostic Centres, Calgary); K. Ugrin and D. Van Why (Bozeman Deaconess Hospital, Bozeman); M. Bauman, A. Price, S. Matinkhah and K. Sanders (University of Utah Hospital, Salt Lake City); S. Merchant, E. Hsu and J. Morgan (HSC Cores Research Facility, University of Utah, Salt Lake City); I. Mitchell and N. Newman (Queensland X-ray, Brisbane); K. Mardon (Centre for Advanced Imaging, The University of Queensland, Brisbane); and R. Lawrence (Queensland Museum, Brisbane). Great thanks are also due to the volunteers who undertook scoring of diaphyseal cancellous bone architecture: R. Lawrence, J. Rasmussen, J. Macmillan, D. O’Boyle and N. Bishop. SCANCO Medical AG kindly provided the scan image presented in Figure 4A, and Figures 1 and 6A were prepared with the assistance of N. Bishop and P. Tierney. The thorough and constructive comments on earlier versions of the manuscript, 
2041 provided by S. Gatesy, T. Ryan, D. Henderson, E. Snively and an anonymous reviewer, are all 2042 greatly appreciated, and substantially improved the clarity and content of the research presented

2043

2044

2045

2046

2047

2048

2049

2050

2051

2052

2053

2054

2055

2056

2057

2058

2059

2060

2061

2062

2063

2064

2065

2066

2067

2068

2069

2070

2071

2072

2073

2074

2075

2076

2077

2078

2079

2080

2081

here. All scripts and data used are held in the Geosciences Collection of the Queensland Museum, and will be made available upon request to the Collections Manager. Additionally, a complete copy of the fossil CT scan data obtained in the present study is accessioned with the respective institutions in which the specimens are housed.

\section{I.8 References}

Aamodt A, Lund-Larsen J, Eine J, Andersen E, Benum P, and Schnell Husby O. 1997. In Vivo Measurements Show Tensile Axial Strain in the Proximal Lateral Aspect of the Human Femur. Journal of Orthopaedic Research 15:927-931.

Abel R, and Macho GA. 2011. Ontogenetic changes in the internal and external morphology of the ilium in modern humans. Journal of Anatomy 218:324-335.

Adachi T, Tsubota K, Tomita Y, and Hollister SJ. 2001. Trabecular Surface Remodeling Simulation for Cancellous Bone Using Microstructural Voxel Finite Element Models. Journal of Biomechanical Engineering 123:403-409.

Alexander RM. 1983. Allometry of the leg bones of moas (Dinornithes) and other birds. Journal of Zoology 200:215-231.

Alexander RM. 1985. Mechanics of posture and gait of some large dinosaurs. Zoological Journal of the Linnean Society 83:1-25.

Alexander RM. 1989. Dynamics of Dinosaurs and Other Extinct Giants. New York: Columbia University Press.

Alexander RM. 1991. Doubts and assumptions in dinosaur mechanics. Interdisciplinary Science Reviews 16:175-181.

Allen V, Bates KT, Li Z, and Hutchinson JR. 2013. Linking the evolution of body shape and locomotor biomechanics in bird-line archosaurs. Nature 497:104-107.

Allen V, Paxton H, and Hutchinson JR. 2009. Variation in Center of Mass Estimates for Extant Sauropsids and its Importance for Reconstructing Inertial Properties of Extinct Archosaurs. The Anatomical Record 292:1442-1461.

Allmendinger RW, Cardozo NC, and Fisher D. 2013. Structural Geology Algorithms: Vectors and Tensors. Cambridge: Cambridge University Press.

Amson E, Arnold P, van Heteren AH, Canoville A, and Nyakatura JA. 2017. Trabecular architecture in the forelimb epiphyses of extant xenarthrans (Mammalia). Frontiers in Zoology 14:52. 
2082

2083

2084

2085

2086

2087

2088

2089

2090

2091

2092

2093

2094

2095

2096

2097

2098

2099

2100

2101

2102

2103

2104

2105

2106

2107

2108

2109

2110

2111

2112

2113

2114

2115

2116

2117

2118

2119

2120

2121

2122

2123

2124

2125

2126

2127

Andrada E, Nyakatura JA, Bergmann F, and Blickhan R. 2013. Adjustments of global and local hindlimb properties during terrestrial locomotion of the common quail (Coturnix coturnix). Journal of Experimental Biology 216:3906-3916.

Bakker RT. 1980. Dinosaur heresy-dinosaur renaissance: Why we need endothermic archosaurs for a comprehensive theory of bioenergetic evolution. In: Thomas RDK, and Olson EC, eds. A Cold Look at the Warm Blooded Dinosaurs. Boulder: Westview Press, Inc.

Bakker RT. 1986. The Dinosaur Heresies. New York: William Morrow \& Company, Inc.

Barak MM, Lieberman DE, and Hublin J-J. 2011. A Wolff in sheep's clothing: Trabecular bone adaptation in response to changes in joint loading orientation. Bone 49:1141-1151.

Barak MM, Lieberman DE, Raichlen DA, Pontzer H, Warrener AG, and Hublin J-J. 2013. Trabecular Evidence for a Human-Like Gait in Australopithecus africanus. PLoS ONE 8:e77687.

Barthel KU. 2006. 3D-Data Representation with ImageJ. Proceedings of the First ImageJ User and Developer Conference. Luxembourg: The Centre de Recherche Public Henri Tudor. p 1-4.

Batdorf SB. 1947. A simplified method of elastic-stability analysis for thin cylindrical shells. II modified equilibrium equation. National Advisory Committee for Aeronautics Technical Note 1342:1-32.

Batdorf SB, Stein M, and Schildcrout M. 1947. Critical stress of thin-walled cylinders in torsion. National Advisory Committee for Aeronautics Technical Note 1344:1-26.

Bates KT, Benson RBJ, and Falkingham PL. 2012. A computational analysis of locomotor anatomy and body mass evolution in Allosauroidea (Dinosauria: Theropoda). Paleobiology 38:486-507.

Baumel JJ, and Witmer LM. 1993. Osteologia. In: Baumel JJ, King AS, Breazile JE, Evans HE, and Vanden Berge JC, eds. Handbook of Avian Anatomy: Nomina Anatomica Avium. 2 ed. Cambridge: Nuttall Ornithological Club, 45-132.

Beaupré GS, Orr TE, and Carter DR. 1990. An Approach for Time-Dependent Bone Modeling and Remodeling - Application: A Preliminary Remodeling Simulation. Journal of Orthopaedic Research 8:662-670.

Beer FP, Johnston ER, Jr, DeWolf JT, and Mazurek DF. 2012. Mechanics of Materials. New York: McGraw-Hill.

Bell PR, and Snively E. 2008. Polar dinosaurs on parade: a review of dinosaur migration. Alcheringa 32:271-284.

Ben-Zvi Y, Reznikov N, Shahar R, and Weiner S. 2017. 3D architecture of Trabecular Bone in the Pig Mandible and Femur: Inter-Trabecular angle Distributions. Frontiers in Materials 4:29.

Bennett PM, and Owens IPF. 2002. Evolutionary Ecology of Birds. Oxford: Oxford University Press.

Benson RBJ, Hunt G, Carrano MT, and Campione NE. in press. Cope's Rule and the adaptive landscape of dinosaur body size evolution. Palaeontology.

Bernsen J. 1986. Dynamic Thresholding of Grey-Level Images. Proceedings of the 8th International Conference on Pattern Recognition. Paris, France. p 1251-1255.

Bertram JEA, and Biewener AA. 1988. Bone curvature: sacrificing strength for load predictability? Journal of Theoretical Biology 131:75-92.

Biewener AA. 1992. Overview of structural mechanics. In: Biewener AA, ed. Biomechanics: Structures and Systems - A Practical Approach. Oxford: Oxford University Press, 1-20. 
2128

2129

2130

2131

2132

2133

2134

2135

2136

2137

2138

2139

2140

2141

2142

2143

2144

2145

2146

2147

2148

2149

2150

2151

2152

2153

2154

2155

2156

2157

2158

2159

2160

2161

2162

2163

2164

2165

2166

2167

2168

2169

2170

2171

Biewener AA, Fazzalari NL, Konieczynski DD, and Baudinette RV. 1996. Adaptive Changes in Trabecular Architecture in Relation to Functional Strain Patterns and Disuse. Bone 19:18.

Biewener AA, Swartz SM, and Bertram JEA. 1986. Bone Modelling During Growth: Dynamics Strain Equilibrium in the Chick Tibiotarsus. Calcified Tissue International 39:390-395.

Biewener AA, and Taylor CR. 1986. Bone strain: a determinant of gait and speed? Journal of Experimental Biology 123:383-400.

Bishop PJ, Clemente CJ, Graham DF, Lamas LP, Hutchinson JR, Rubenson J, Hancock JA, Wilson RS, Hocknull SA, Barrett RS, and Lloyd DG. 2018. The Influence of Speed and Size on Avian Terrestrial Locomotor Biomechanics: Predicting Locomotion in Extinct Theropod Dinosaurs. PLoS ONE 13:e0192172.

Bishop PJ, Clemente CJ, Hocknull SA, Barrett RS, and Lloyd DG. 2017. The effects of cracks on the quantification of the cancellous bone fabric tensor in fossil and archaeological specimens: a simulation study. Journal of Anatomy 230:461-470.

Bishop PJ, Hocknull SA, Clemente CJ, Hutchinson JR, Barrett RS, and Lloyd DG. in review-a. Cancellous bone architecture and theropod dinosaur locomotion. Part II - A new approach to reconstructing posture and locomotor biomechanics in extinct tetrapod vertebrates. PeerJ.

Bishop PJ, Hocknull SA, Clemente CJ, Hutchinson JR, Farke AA, Barrett RS, and Lloyd DG. in review-b. Cancellous bone architecture and theropod dinosaur locomotion. Part III Inferring posture and locomotor biomechanics in extinct theropods, and its evolution on the line to birds. PeerJ.

Bishop PJ, Walmsley CW, Phillips MJ, Quayle MR, Boisvert CA, and McHenry CR. 2015. Oldest Pathology in a Tetrapod Bone Illuminates the Origin of Terrestrial Vertebrates. PLoS ONE 10:e0125723.

Blob RW, and Biewener AA. 1999. In vivo locomotor strain in the hindlimb bones of Alligator mississippiensis and Iguana iguana: implications for the evolution of limb bone safety factor and non-sprawling limb posture. Journal of Experimental Biology 202:1023-1046.

Blob RW, and Biewener AA. 2001. Mechanics of limb bone loading during terrestrial locomotion in the green iguana (Iguana iguana) and American alligator (Alligator mississippiensis). Journal of Experimental Biology 204:1099-1122.

Blomberg SP, and Garland T, Jr. 2002. Tempo and mode in evolution: phylogenetic inertia, adaptation and comparative methods. Journal of Evolutionary Biology 15:899-910.

Bonnan MF, Sandrik JL, Nishiwaki T, Wilhite DR, Elsey RM, and Vittore C. 2010. Calcified Cartilage Shape in Archosaur Long Bones Reflects Overlying Joint Shape in StressBearing Elements: Implications for Nonavian Dinosaur Locomotion. The Anatomical Record 293:2044-2055.

Boyle C, and Kim IY. 2011. Three-dimensional micro-level computational study of Wolff's law via trabecular bone remodeling in the human proximal femur using design space topology optimization. Journal of Biomechanics 44:935-942.

Brassey CA, Kitchener AC, Withers PJ, Manning PL, and Sellers WI. 2013. The Role of CrossSectional Geometry, Curvature, and Limb Posture in Maintaining Equal Safety Factors: A Computed Tomography Study. The Anatomical Record 296:395-413.

Brusatte SL. 2012. Dinosaur Paleobiology. West Sussex: John Wiley and Sons, Ltd. 
2172

2173

2174

2175

2176

2177

2178

2179

2180

2181

2182

2183

2184

2185

2186

2187

2188

2189

2190

2191

2192

2193

2194

2195

2196

2197

2198

2199

2200

2201

2202

2203

2204

2205

2206

2207

2208

2209

2210

2211

2212

2213

2214

2215

2216

Butcher MT, Espinoza NR, Cirilo SR, and Blob RW. 2008. In vivo strains in the femur of river cooter turtles (Pseudemys concinna) during terrestrial locomotion: tests of force-platform models of loading mechanics. Journal of Experimental Biology 211:2397-2407.

Cardozo NC, and Allmendinger RW. 2013. Spherical projections with OSXStereonet. Computers \& Geosciences 51:193-205.

Carlson KJ, Doran-Sheehy DM, Hunt KD, Nishida T, Yamanaka A, and Boesch C. 2006. Locomotor behaviour and long bone morphology in individual free-ranging chimpanzees. Journal of Human Evolution 50:394-404.

Carrano MT. 1998. Locomotion in non-avian dinosaurs: integrating data from hindlimb kinematics, in vivo strains, and bone morphology. Paleobiology 24:450-469.

Carrano MT. 2000. Homoplasy and the evolution of dinosaur locomotion. Paleobiology 26:489512.

Carrano MT. 2001. Implications of limb bone scaling, curvature and eccentricity in mammals and non-avian dinosaurs. Journal of Zoology 254:41-55.

Carrano MT. 2005. The Evolution of Sauropod Locomotion: Morphological Diversity of a Secondarily Quadrupedal Radiation. In: Curry Rogers KA, and Wilson JA, eds. The Sauropods: Evolution and Paleobiology. Berkeley: University of California Press, 229251.

Carrano MT. 2006. Body-Size Evolution in the Dinosauria. In: Carrano MT, Gaudin TJ, Blob RW, and Wible JR, eds. Amniote Paleobiology: Perspectives on the Evolution of Mammals, Birds, and Reptiles. Chicago: University of Chicago Press, 225-268.

Carrano MT, Benson RBJ, and Sampson SD. 2012. The phylogeny of Tetanurae (Dinosauria: Theropoda). Journal of Systematic Palaeontology 10:211-300.

Carrano MT, and Biewener AA. 1999. Experimental Alteration of Limb Posture in the Chicken (Gallus gallus) and its Bearing on the Use of Birds as Analogs for Dinosaur Locomotion. Journal of Morphology 240:237-249.

Carrano MT, and Hutchinson JR. 2002. Pelvic and hindlimb musculature of Tyrannosaurus rex (Dinosauria: Theropoda). Journal of Morphology 253:207-228.

Carter DR, and Beaupré GS. 2001. Skeletal Function and Form: Mechanobiology of Skeletal Development, Aging, and Regeneration. Cambridge: Cambridge University Press.

Carter DR, Orr TE, and Fyhrie DP. 1989. Relationships between loading history and femoral cancellous bone architecture. Journal of Biomechanics 22:231-244.

Chiappe LM, and Witmer LM. 2002. Mesozoic Birds: Above the Heads of the Dinosaurs. Berkeley: University of California Press.

Chitale AK, and Gupta RC. 2011. Product Design and Manufacturing. New Delhi: PHI Learning Private Limited.

Christen P, Ito K, Ellouz R, Boutroy S, Sornay-Rendu E, Chapurlat RD, and van Rietbergen B. 2014. Bone remodelling in humans is load-driven but not lazy. Nature Communications 5:4855.

Christiansen M, Kveiborg M, Kassem M, Clark BFC, and Rattan SIS. 2000. CBFA1 and Topoisomerase I mRNA Levels Decline During Cellular Aging of Human Trabecular Osteoclasts. Journal of Gerontology 55A:B194-B200.

Christiansen P. 1997. Locomotion in sauropod dinosaurs. Gaia 14:45-75.

Christiansen P. 1998. Strength indicator values of theropod long bones, with comments on limb proportions and cursorial potential. Gaia 15:241-255. 
2217 Christiansen P. 1999. Long bone scaling and limb posture in non-avian theropods: evidence for

2218

2219

2220

2221

2222

2223

2224

2225

2226

2227

2228

2229

2230

2231

2232

2233

2234

2235

2236

2237

2238

2239

2240

2241

2242

2243

2244

2245

2246

2247

2248

2249

2250

2251

2252

2253

2254

2255

2256

2257

2258

2259

2260

2261 differential allometry. Journal of Vertebrate Paleontology 19:666-680.

Clarke B. 2008. Normal Bone Anatomy and Physiology. Clinical Journal of the American Society of Nephrology 3:S131-S139.

Clemente CJ, Withers PC, Thompson GG, and Lloyd DG. 2013. Lizard tricks: overcoming conflicting requirements of speed versus climbing ability by altering biomechanics of the lizard stride. Journal of Experimental Biology 216:3854-3862.

Coelho PG, Fernandes PR, Rodrigues HC, Cardoso JB, and Guedes JM. 2009. Numerical modeling of bone tissue adaptation - A hierarchical approach for bone apparent density and trabecular structure. Journal of Biomechanics 42:830-837.

Coombs WP, Jr. 1978. Theoretical aspects of cursorial adaptations in dinosaurs. Quarterly Review of Biology 53:393-418.

Cowin SC. 1986. Wolff's Law of Trabecular Architecture at Remodelling Equilibrium. Journal of Biomechanical Engineering 108:83-88.

Cowin SC. 1997. Remarks on the paper entitled 'Fabric and elastic principal directions of cancellous bone are closely related'. Journal of Biomechanics 30:1191-1192.

Cowin SC. 2001. The false premise in Wolff's Law. In: Cowin SC, ed. Bone Biomechanics Handbook. 2 ed. Boca Raton: CRC Press, 30-31-30-15.

Cowin SC, Sadegh AM, and Luo GM. 1992. An Evolutionary Wolff's Law for Trabecular Architecture. Journal of Biomechanical Engineering 114:129-136.

Cracraft J. 1971. The functional morphology of the hind limb of the domestic pigeon, Columba livia. Bulletin of the American Museum of Natural History 144:171-268.

Cresswell EN, Goff MG, Nguyen TM, Lee WX, and Hernandez CJ. 2016. Spatial relationships between bone formation and mechanical stress within cancellous bone. Journal of Biomechanics 49:222-228.

Cruz-Orive LM, Karlsson LM, Larsen SE, and Wainschtein F. 1992. Characterizing anisotropy: a new concept. Micron and Microscopica Acta 23:75-76.

Cubo J, Woodward H, Wolff E, and Horner JR. 2015. First Reported Cases of Biomechanically Adaptive Bone Modeling in Non-Avian Dinosaurs. PLoS ONE 10:e131131.

Cunningham CA, and Black SM. 2009a. Anticipating bipedalism: trabecular organization in the newborn ilium. Journal of Anatomy 214:817-829.

Cunningham CA, and Black SM. 2009b. Development of the fetal ilium - challenging concepts of bipedality. Journal of Anatomy 214:91-99.

Currey JD. 2002. Bones: Structure and Mechanics. Princeton: Princeton University Press.

Currey JD. 2003. The many adaptations of bone. Journal of Biomechanics 36:1487-1495.

D’Anastasio R, Wroe S, Tuniz C, Mancini L, Cesana DT, Dreossi D, Ravichandiran M, Attard M, Parr WCH, Agur A, and Capasso L. 2013. Micro-Biomechanics of the Kebara 2 Hyoid and Its Implications for Speech in Neanderthals. PLoS ONE 8:e82261.

Dacke CG, Arkle S, Cook DJ, Wormstone IM, Jones S, Zaidi M, and Bascal ZA. 1993. Medullary bone and avian calcium regulation. Journal of Experimental Biology 184:6388.

Davies SJJF. 2002. Ratites and Tinamous. Oxford: Oxford University Press.

de Ricqlès AJ. 1976. On bone histology of fossil and living reptiles, with comments on its functional and evolutionary importance. In: Bellairs AdA, and Cox CB, eds. Morphology and Biology of Reptiles. London: Academic Press, 123-150. 
2262 Demes B. 2007. In vivo bone strain and bone functional adaptation. American Journal of

2263

2264

2265

2266

2267

2268

2269

2270

2271

2272

2273

2274

2275

2276

2277

2278

2279

2280

2281

2282

2283

2284

2285

2286

2287

2288

2289

2290

2291

2292

2293

2294

2295

2296

2297

2298

2299

2300

2301

2302

2303

2304

2305

2306

2307 Physical Anthropology 133:717-722.

Demes B, Qin Y-X, Stern JT, Jr, Larson SG, and Rubin CT. 2001. Patterns of strain in the macaque tibia during functional activity. American Journal of Physical Anthropology 116:257-265.

Demes B, Stern JT, Jr, Hausman MR, Larson SG, McLeod KJ, and Rubin CT. 1998. Patterns of strain in the macaque ulna during functional activity. American Journal of Physical Anthropology 106:87-100.

DeSilva JM, and Devlin MJ. 2012. A comparative study of the trabecular bony architecture of the talus in humans, non-human primates, and Australopithecus. Journal of Human Evolution 63:536-551.

Donnell LH. 1933. Stability of thin-walled tubes under torsion. National Advisory Committee for Aeronautics Technical Report 479:95-116.

Doube M, Kłosowski MM, Arganda-Carreras I, Cordelières FP, Dougherty RP, Jackson JS, Schmid B, Hutchinson JR, and Shefelbine SJ. 2010. BoneJ: Free and extensible bone image analysis in ImageJ. Bone 47:1076-1079.

Doube M, Yen SCW, Kłosowski MM, Farke AA, Hutchinson JR, and Shefelbine SJ. 2012. Whole-bone scaling of the avian pelvic limb. Journal of Anatomy 221:21-29.

Dunning JB, Jr. 2007. CRC Handbook of Avian Body Masses. Boca Raton: CRC Press.

Elke RPE, Cheal EJ, Simmons C, and Poss R. 1995. Three-Dimensional Anatomy of the Cancellous Structures within the Proximal Femur from Computed Tomography Data. Journal of Orthopaedic Research 13:513-523.

Enlow DH. 1969. The Bone of Reptiles. In: Gans C, Bellairs AdA, and Parsons TS, eds. Biology of the Reptilia. London: Academic Press, 45-80.

Fariña RA, Vizcaíno SF, and Blanco RE. 1997. Scaling of the indicator of athletic capability in fossil and extant land tetrapods. Journal of Theoretical Biology 185:441-446.

Farke AA, and Alicea J. 2009. Femoral strength and posture in terrestrial birds and non-avian theropods. The Anatomical Record 292:1406-1411.

Farlow JO, Chapman RE, Breithaupt BH, and Matthews N. 2012. The Scientific Study of Dinosaur Footprints. In: Brett-Surman MK, Holtz TR, Jr, and Farlow JO, eds. The Complete Dinosaur. 2 ed. Bloomington: Indiana University Press, 712-759.

Farlow JO, Gatesy SM, Holtz TR, Jr, Hutchinson JR, and Robinson JM. 2000. Theropod Locomotion. American Zoologist 40:640-663.

Farlow JO, Smith MB, and Robinson JM. 1995. Body mass, bone "strength indicator," and cursorial potential of Tyrannosaurus rex. Journal of Vertebrate Paleontology 15:713725.

Frost HM. 1987. Bone mass and the mechanostat - a proposal. The Anatomical Record 219:1-9.

Frost HM. 2003. Bone's mechanostat: a 2003 update. The Anatomical Record 275A:1081-1101.

Fyhrie DP, and Carter DR. 1986. A Unifying Principle Relating Stress to Trabecular Bone Morphology. Journal of Orthopaedic Research 4:304-317.

Garden RS. 1961. The structure and function of the proximal end of the femur. Journal of Bone and Joint Surgery 43B:576-589.

Gatesy SM. 1990. Caudofemoral musculature and the evolution of theropod locomotion. Paleobiology 16:170-186.

Gatesy SM. 1991a. Hind limb movements of the American alligator (Alligator mississippiensis) and postural grades. Journal of Zoology 224:577-588. 
2308 Gatesy SM. 1991b. Hind Limb Scaling in Birds and Other Theropods: Implications for

2309

2310

2311

2312

2313

2314

2315

2316

2317

2318

2319

2320

2321

2322

2323

2324

2325

2326

2327

2328

2329

2330

2331

2332

2333

2334

2335

2336

2337

2338

2339

2340

2341

2342

2343

2344

2345

2346

2347

2348

2349

2350

2351

2352
Terrestrial Locomotion. Journal of Morphology 209:83-96.

Gatesy SM. 1995. Functional evolution of the hindlimb and tail from basal theropods to birds. In: Thomason JJ, ed. Functional Morphology in Vertebrate Paleontology. New York: Cambridge University Press, 219-234.

Gatesy SM. 1999. Guineafowl Hindlimb Function I: Cineradiographic Analysis and Speed Effects. Journal of Morphology 240:115-125.

Gatesy SM. 2002. Locomotor Evolution on the Line to Modern Birds. In: Chiappe LM, and Witmer LM, eds. Mesozoic Birds: Above the Heads of the Dinosaurs. Berkeley: University of California Press, 432-447.

Gatesy SM, Bäker M, and Hutchinson JR. 2009. Constraint-based exclusion of limb poses for reconstructing theropod dinosaur locomotion. Journal of Vertebrate Paleontology 29:535-544.

Gatesy SM, and Biewener AA. 1991. Bipedal locomotion: effects of speed, size and limb posture in birds and humans. Journal of Zoology 224:127-147.

Gatesy SM, and Middleton KM. 1997. Bipedalism, flight, and the evolution of theropod locomotor diversity. Journal of Vertebrate Paleontology 17:308-329.

Gatesy SM, Middleton KM, Jenkins FA, Jr, and Shubin NH. 1999. Three-dimensional preservation of foot movements in Triassic theropod dinosaurs. Nature 399:141-144.

Gauthier JA. 1986. Saurischian monophyly and the origin of birds. Memoirs of the California Academy of Sciences 8:1-55.

Gefen A, and Seliktar R. 2004. Comparison of the trabecular architecture and the isostatic stress flow in the human calcaneus. Medical Engineering \& Physics 26:119-129.

Georgiou L, Kivell TL, Pahr DH, and Skinner MM. 2018. Trabecular bone patterning in the hominoid distal femur. PeerJ 6:e5156.

Giddings VL, Beaupré GS, Whalen RT, and Carter DR. 2000. Calcaneal loading during walking and running. Medicine \& Science in Sports \& Exercise 32:627-634.

Gillette DD, and Lockley MG. 1989. Dinosaur Tracks and Traces. Cambridge: Cambridge University Press.

Goldstein SA, Goulet R, and McCubbrey D. 1993. Measurement and Significance of ThreeDimensional Architecture to the Mechanical Integrity of Trabecular Bone. Calcified Tissue International 53 (suppl. 1):S127-S133.

Goldstein SA, Matthews LS, Kuhn JL, and Hollister SJ. 1991. Trabecular bone remodelling: an experimental model. Journal of Biomechanics 24 (suppl. 1):135-150.

Gorissen BMC, Wolschrijn CF, van Vilsteren AAM, van Rietbergen B, and van Weeren PR. 2016. Trabecular Bone of Precocials at Birth; Are They Prepared to Run for the Wolf(f)? Journal of Morphology 277:948-956.

Gosman JH, and Ketcham RA. 2009. Patterns in Ontogeny of Human Trabecular Bone From SunWatch Village in the Prehistoric Ohio Valley: General Features of Microarchitectural Change. American Journal of Physical Anthropology 138:318-332.

Gosman JH, Stout SD, and Larsen CS. 2011. Skeletal Biology Over the Life Span: A View From the Surfaces. Yearbook of Physical Anthropology 54:86-98.

Gross T, Kivell TL, Skinner MM, Nguyen NH, and Pahr DH. 2014. A CT-image-based framework for the holistic analysis of cortical and trabecular bone morphology. Palaeontologia Electronica 17:33A. 
2353

2354

2355

2356

2357

2358

2359

2360

2361

2362

2363

2364

2365

2366

2367

2368

2369

2370

2371

2372

2373

2374

2375

2376

2377

2378

2379

2380

2381

2382

2383

2384

2385

2386

2387

2388

2389

2390

2391

2392

2393

2394

2395

2396

2397

2398
Grossi B, Iriarte-Díaz J, Larach O, Canals M, and Vásquez RA. 2014. Walking Like Dinosaurs: Chickens with Artificial Tails Provide Clues about Non-Avian Theropod Locomotion. PLoS ONE 9:e88458.

Hackett SJ, Kimball RT, Reddy S, Bowie RCK, Braun EL, Braun MJ, Chojnowski JL, Cox WA, Han K-L, Harshman J, Huddleston CJ, Marks BD, Miglia KJ, Moore WS, Sheldon FH, Steadman DW, Witt CC, and Yuri T. 2008. A Phylogenomic Study of Birds Reveals Their Evolutionary History. Science 320:1763-1768.

Haddrath O, and Baker AJ. 2012. Multiple nuclear genes and retroposons support vicariance and dispersal of the palaeognaths, and an Early Cretaceous origin of modern birds.

Proceedings of the Royal Society of London, Series B 279:4617-4625.

Hammer Ø, Harper DAT, and and Ryan PD. 2001. PAST: Paleontological Statistics Software Package for Education and Data Analysis. Palaeontologia Electronica 4:4.

Harrigan TP, Jasty M, Mann RW, and Harris WH. 1988. Limitations of the continuum assumption in cancellous bone. Journal of Biomechanics 21:269-275.

Havill LM, Allen MR, Bredbenner TL, Burr DB, Nicolella DP, Turner CH, Warren DM, and Mahaney MC. 2010. Heritability of lumbar trabecular bone mechanical properties in baboons. Bone 46:835-840.

Hayes WC, and Snyder B. 1981. Toward a quantitative formulation of Wolff's Law in trabecular bone. In: Cowin SC, ed. Mechanical Properties of Bone. New York: American Society of Mechanical Engineers, 43-68.

Hébert D, Lebrun R, and Marivaux L. 2012. Comparative Three-Dimensional Structure of the Trabecular Bone in the Talus of Primates and Its Relationship to Ankle Joint Loads Generated During Locomotion. The Anatomical Record 295:2069-2088.

Heers AM, and Dial KP. 2012. From extant to extinct: locomotor ontogeny and the evolution of avian flight. Trends in Ecology and Evolution 27:296-305.

Heinrich RE, Ruff CB, and Weishampel DB. 1993. Femoral ontogeny and locomotor biomehcnaics of Dryosaurus lettowvorbecki (Dinosauria, Iguanodontia). Zoological Journal of the Linnean Society 108:179-196.

Henderson DM. 1999. Estimating the masses and centers of mass of extinct animals by 3-D mathematical slicing. Paleobiology 25:88-106.

Heřt J. 1994. A new attempt at the interpretation of the functional architecture of the cancellous bone. Journal of Biomechanics 27:239-242.

Holtz TR, Jr. 2012. Theropods. In: Brett-Surman MK, Holtz TR, Jr, and Farlow JO, eds. The Complete Dinosaur. 2 ed. Bloomington: Indiana University Press, 346-378.

Hone DWE, Farke AA, and Wedel MJ. 2016. Ontogeny and the fossil record: what, if anything, is an adult dinosaur? Biology Letters 12:20150947.

Horner JR. 2000. Dinosaur Reproduction and Parenting. Annual Review of Earth and Planetary Sciences 28:19-45.

Horner JR, and Lessem D. 1993. The Complete T. rex. New York: Simon and Schuster, Inc.

Hotton NH, III. 1980. An Alternative to Dinosaur Endothermy: The Happy Wanderers. In: Thomas RDK, and Olson EC, eds. A Cold Look at the Warm-Blooded Dinosaurs. Boulder: Westview Press, Inc., 311-350.

Hübner TR. 2012. Bone Histology in Dysalotosaurus lettowvorbecki (Ornithischia: Iguanodontia) - Variation, Growth, and Implications. PLoS ONE 7:e29958.

Huiskes R. 2000. If bone is the answer, then what is the question? Journal of Anatomy 197:145156. 
2399

2400

2401

2402

2403

2404

2405

2406

2407

2408

2409

2410

2411

2412

2413

2414

2415

2416

2417

2418

2419

2420

2421

2422

2423

2424

2425

2426

2427

2428

2429

2430

2431

2432

2433

2434

2435

2436

2437

2438

2439

2440

2441

2442

2443

2444

Huiskes R, Ruimerman R, van Lenthe GH, and Janssen JD. 2000. Effects of mechanical forces on maintenance and adaptation of form in trabecular bone. Nature 405:704-706.

Hutchinson JR. 2001a. The evolution of femoral osteology and soft tissues on the line to extant birds (Neornithes). Zoological Journal of the Linnean Society 131:169-197.

Hutchinson JR. 2001b. The evolution of pelvic osteology and soft tissues on the line to extant birds (Neornithes). Zoological Journal of the Linnean Society 131:123-168.

Hutchinson JR. 2002. The evolution of hindlimb tendons and muscles on the line to crown-group birds. Comparative Biochemistry and Physiology, Part A 133:1051-1086.

Hutchinson JR. 2006. The evolution of locomotion in archosaurs. Comptes Rendus Palevol 5:519-530.

Hutchinson JR, and Allen V. 2009. The evolutionary continuum of limb function from early theropods to birds. Naturwissenschaften 96:423-448.

Hutchinson JR, Anderson FC, Blemker SS, and Delp SL. 2005. Analysis of hindlimb muscle moment arms in Tyrannosaurus rex using a three-dimensional musculoskeletal computer model: implications for stance, gait, and speed. Paleobiology 31:676-701.

Hutchinson JR, Bates KT, Molnar J, Allen V, and Makovicky PJ. 2011. A Computational Analysis of Limb and Body Dimensions in Tyrannosaurus rex with Implications for Locomotion, Ontogeny and Growth. PLoS ONE 6:e26037.

Hutchinson JR, and Gatesy SM. 2000. Adductors, abductors, and the evolution of archosaur locomotion. Paleobiology 26:734-751.

Hutchinson JR, and Gatesy SM. 2006. Dinosaur locomotion: Beyond the bones. Nature 440:292294.

Hutchinson JR, Miller CE, Fritsch G, and Hildebrandt T. 2008. The Anatomical Foundation for Multidisciplinary Studies of Animal Limb Function: Examples from Dinosaur and Elephant Limb Imaging Studies. In: Endo H, and Frey R, eds. Anatomical Imaging: Towards a New Morphology. Tokyo: Springer, 23-38.

Jacobs CR. 2000. The mechanobioogy of cancellous bone structural adaptation. Journal of Rehabilitation Research and Development 37:209-216.

Jacobs CR, Simo JC, Beaupré GS, and Carter DR. 1997. Adaptive bone remodeling incorporating simultaneous density and anisotropy considerations. Journal of Biomechanics 30:603-613.

Jang IG, and Kim IY. 2008. Computational study of Wolff's law with trabecular architecture in the human proximal femur using topology optimization. Journal of Biomechanics 41:2353-2361.

Jang IG, and Kim IY. 2010a. Application of design space optimization to bone remodeling simulation of trabecular architecture in human proximal femur for higher computational efficiency. Finite Elements in Analysis and Design 46:311-319.

Jang IG, and Kim IY. 2010b. Computational simulation of cortical and trabecular bone change in human proximal femur during bone remodelling. Journal of Biomechanics 43:294-301.

Jarvis ED, Mirarab S, Aberer AJ, Li B, Houde P, Li C, Ho SYW, Faircloth BC, Nabholz B, Howard JT, Suh A, Weber CC, da Fonseca RR, Li J, Zhang F, Li H, Zhou L, Narula N, Liu L, Ganapathy G, Boussau B, Bayzid MS, Zavidovych V, Subramanian S, Gabalbón T, Capella-Gutiérrez S, Huerta-Cepas J, Rekepalli B, Munch K, Schierup M, Lindow B, Warren WC, Ray D, Green RE, Bruford MW, Zhan X, Dixon A, Li S, Li N, Huang Y, Derryberry EP, Bertelsen FF, Sheldon FH, Brumfield RT, Mello CV, Lovell PV, Wirthlin M, Schneider MPC, Prosdocimi F, Samaniego JA, Velazquez AMV, Alfaro-Núñez A, 
Campos PF, Petersen B, Sicheritz-Ponten T, Pas A, Bailey T, Scofield RP, Bunce M, Lambert DM, Zhou Q, Perelman P, Driskell AC, Shapiro B, Xiong Z, Zeng Y, Liu S, Li Z, Liu B, Wu K, Xiao J, Yinqi X, Zheng Q, Zhang Y, Yang H, Wang J, Smeds L, Rheindt FE, Braun M, Fjeldsa J, Orlando L, Barker FK, Jønsson KA, Johnson W, Koepfli K-P, O'Brien S, Haussler D, Ryder OA, Rahbek C, Willerslev E, Graves GR, Glenn TC, McCormack J, Burt D, Ellegren H, Alström P, Edwards SV, Stamatakis A, Mindell DP, Cracraft J, Braun EL, Warnow T, Jun W, Gilbert MTP, and Zhang G. 2014. Wholegenome analyses resolve early branches in the tree of life of modern birds. Science 346:1320-1331.

Jetz W, Thomas GH, Joy JB, Hartmann K, and Mooers AO. 2012. The global diversity of birds in space and time. Nature 491:444-448.

Judex S, Garman R, Squire M, Donahue L-R, and Rubin CT. 2004. Genetically Based Influences on the Site-Specific Regulation of Trabecular and Cortical Bone Morphology. Journal of Bone and Mineral Research 19:600-606.

Kabel J, van Rietbergen B, Odgaard A, and Huiskes R. 1999. Constitutive Relationships of Fabric, Density, and Elastic Properties in Cancellous Bone Architecture. Bone 25:481486.

Kambic RE, Roberts TJ, and Gatesy SM. 2014. Long-axis rotation: a missing degree of freedom in avian bipedal locomotion. Journal of Experimental Biology 217:2770-2782.

Kambic RE, Roberts TJ, and Gatesy SM. 2015. Guineafowl with a twist: asymmetric limb control in steady bipedal locomotion. Journal of Experimental Biology 218:3836-3844.

Kamibayashi L, Wyss UP, Cooke TDV, and Zee B. 1995. Changes in Mean Trabecular Orientation in the Medial Condyle of the Proximal Tibia in Osteoarthritis. Calcified Tissue International 57:69-73.

Keaveny TM, Morgan EF, Niebur GL, and Yeh OC. 2001. Biomechanics of Trabecular Bone. Annual Review of Biomedical Engineering 3:307-333.

Keaveny TM, and Yeh OC. 2002. Architecture and trabecular bone - Toward an improved understanding of the biomechanical effects of age, sex and osteoporosis. Journal of Musculoskeletal and Neuronal Interactions 2:205-208.

Ketcham RA, and Carlson WD. 2001. Acquisition, optimization and interpretation of X-ray computed tomographic imagery: applications to the geosciences. Computers \& Geosciences 27:381-400.

Ketcham RA, and Ryan TM. 2004. Quantification and visualization of anisotropy in trabecular bone. Journal of Microscopy 213:158-171.

Kivell TL. 2016. A review of trabecular bone functional adaptation: what have we learned from trabecular analyses in extant hominoids and what can we apply to fossils? Journal of Anatomy 228:569-594.

Koch JC. 1917. The laws of bone architecture. American Journal of Anatomy 21:177-298.

Koo TK, and Li MY. 2016. A Guideline of Selecting and Reporting Intraclass Correlation Coefficients for Reliability Research. Journal of Chiropractic Medicine 15:155-163.

Kowalczyk P. 2010. Simulation of orthotropic microstructure remodelling of cancellous bone. Journal of Biomechanics 43:563-569.

Ksepka DT. 2009. Broken gears in the avian molecular clock: new phylogenetic analyses support stem galliform status for Gallinuloides wyomingensis and rallid affinities for Amitabha urbsinterdictensis. Cladistics 25:173-197. 
2490

2491

2492

2493

2494

2495

2496

2497

2498

2499

2500

2501

2502

2503

2504

2505

2506

2507

2508

2509

2510

2511

2512

2513

2514

2515

2516

2517

2518

2519

2520

2521

2522

2523

2524

2525

2526

2527

2528

2529

2530

2531

2532

2533

2534

2535

Ksepka DT, and Phillips MJ. 2015. Avian diversification patterns across the K-Pg boundary: influence of calibrations, datasets, and model misspecification. Annals of the Missouri Botanic Garden 100:300-328.

Lambers FM, Koch K, Kuhn G, Ruffoni D, Weigt C, Schulte FA, and Müller R. 2013. Trabecular bone adapts to long-term cyclic loading by increasing stiffness and normalization of dynamic morphometric rates. Bone 55:325-334.

Landini G. 2008. Advanced shape analysis with ImageJ. Proceedings of the Second ImageJ User and Developer Conference. Luxembourg: The Centre de Recherche Public Henri Tudor. p 116-121.

Landini G, Randell DA, Fouad S, and Galton A. 2016. Automatic thresholding from the gradients of region boundaries. Journal of Microscopy In Press.

Lane JM, Riley EH, and Wirganowicz PZ. 1996. Osteoporosis: Diagnosis and Treatment. Journal of Bone and Joint Surgery 78A:618-166.

Lanyon LE. 1974. Experimental support for the trajectorial theory of bone structure. Journal of Bone and Joint Surgery 56B:160-166.

Lanyon LE. 1996. Locomotor Loading and Functional Adaptation in Limb Bones. Symposium of the Zoological Society of London 48:305-329.

Lanyon LE, Hampson WGJ, Goodship AE, and Shah JS. 1975. Bone deformation recorded in vivo from strain gauges attached to the human tibial shaft. Acta Orthopaedica Scandinavica 46:256-268.

Lee AH, and Werning S. 2008. Sexual maturity in growing dinosaurs does not fit reptilian growth models. Proceedings of the National Academy of Sciences 105:582-587.

Lee MSY, Cau A, Naish D, and Dyke GJ. 2014. Sustained miniaturization and anatomical innovation in the dinosaurian ancestors of birds. Science 345:562-566.

Legendre P, and Legendre L. 2012. Numerical Ecology, Third English Edition. Amsterdam: Elsevier.

Lieberman DE, Pearson OM, Polk JD, Demes B, and Crompton AW. 2003. Optimization of bone growth and remodelling in response to loading in tapered mammalian limbs. Journal of Experimental Biology 206:3125-3138.

Lieberman DE, Polk JD, and Demes B. 2004. Predicting Long Bone Loading From CrossSectional Geometry. American Journal of Physical Anthropology 123:156-171.

Lockley MG. 1991. Tracking Dinosaurs. Cambridge: Cambridge University Press.

Lovejoy CO, Meindl RS, Ohman JC, Heiple KG, and White TD. 2002. The Maka Femur and Its Bearing on the Antiquity of Human Walking: Applying Contemporary Concepts of Morphogenesis to the Human Fossil Record. American Journal of Physical Anthropology 119:97-133.

Macchiarelli R, Bondioli L, Galichon V, and Tobias PV. 1999. Hip bone trabecular architecture shows uniquely distinctive locomotor behaviour in South African australopithecines. Journal of Human Evolution 36:211-232.

Maidment SCR, and Barrett PM. 2014. Osteological correlates for quadrupedality in ornithischian dinosaurs. Acta Palaeontologica Polonica 59:53-70.

Maidment SCR, Bates KT, Falkingham PL, VanBuren C, Arbour V, and Barrett PM. 2014. Locomotion in ornithischian dinosaurs: an assessment using three-dimensional computational modelling. Biological Reviews 89:588-617.

Maidment SCR, Linton DH, Upchurch P, and Barrett PM. 2012. Limb-Bone Scaling Indicates Diverse Stance and Gait in Quadrupedal Ornithischian Dinosaurs. PLoS ONE 7:e36904. 
2536

2537

2538

2539

2540

2541

2542

2543

2544

2545

2546

2547

2548

2549

2550

2551

2552

2553

2554

2555

2556

2557

2558

2559

2560

2561

2562

2563

2564

2565

2566

2567

2568

2569

2570

2571

2572

2573

2574

2575

2576

2577

2578

2579

2580

2581

Main RP, and Biewener AA. 2004. Ontogenetic patterns of limb loading, in vivo bone strains and growth in the goat radius. Journal of Experimental Biology 207:2577-2588.

Main RP, and Biewener AA. 2007. Skeletal strain patterns and growth in the emu hindlimb during ontogeny. Journal of Experimental Biology 210:2676-2690.

Mallison H. 2010a. CAD assessment of the posture and range of motion of Kentrosaurus aethiopicus Hennig 1915. Swiss Journal of Geosciences 103:211-233.

Mallison H. 2010b. The digital Plateosaurus II: An assessment of the range of motion of the limbs and vertebral column and of previous reconstructions using a digital skeletal mount. Acta Palaeontologica Polonica 55:433-458.

Maquer G, Musy SN, Wandel J, Gross T, and Zysset PK. 2015. Bone Volume Fraction and Fabric Anisotropy Are Better Determinants of Trabecular Bone Stiffness than Other Morphological Variables. Journal of Bone and Mineral Research 30:1000-1008.

Martin RB, Burr DB, and Sharkey NA. 1998. Skeletal Tissue Mechanics. New York: Springer.

Matarazzo SA. 2015. Trabecular Architecture of the Manual Elements Reflects Locomotor Patterns in Primates. PLoS ONE 10:e0120436.

Mazzetta GV, Fariña RA, and Vizcaíno SF. 1998. On the palaeobiology of the South American horned theropod Carnotaurus sastrei Bonaparte. Gaia 15:185-192.

Middleton KM, and Gatesy SM. 2000. Theropod forelimb design and evolution. Zoological Journal of the Linnean Society 128:149-187.

Miller Z, Fuchs MB, and Arcan M. 2002. Trabecular bone adaptation with an orthotropic material model. Journal of Biomechanics 35:247-256.

Mitchell KJ, Llamas B, Soubrier J, Rawlence NJ, Worthy TH, Wood J, Lee MSY, and Cooper A. 2014. Ancient DNA reveals elephant birds and kiwi are sister taxa and clarifies ratite bird evolution. Science 344:898-900.

Mittra E, Rubin CT, and Qin Y-X. 2005. Interrelationship of trabecular mechanical and microstructural properties in sheep trabecular bone. Journal of Biomechanics 38:12291237.

Molnar RE, and Farlow JO. 1990. Carnosaur Paleobiology. In: Weishampel DB, Dodson P, and Osmólska H, eds. The Dinosauria. 1 ed. Berkeley: University of California Press, 210224.

Moreno K, Carrano MT, and Snyder R. 2007. Morphological Changes in Pedal Phalanges Through Ornithopod Dinosaur Evolution: A Biomechanical Approach. Journal of Morphology 268:50-63.

Morgan-Richards M, Trewick SA, Bartosch-Härlid A, Kardailsky O, Phillips MJ, McLenachan P, and Penny D. 2008. Bird evolution: testing the Metaves clade with six new mitochondrial genomes. BMC Evolutionary Biology 8:20.

Mullender MG, and Huiskes R. 1995. Proposal for the Regulatory Mechanism of Wolff's Law. Journal of Orthopaedic Research 13:503-512.

Murray PDF. 1936. Bones: A Study of the Development and Structure of the Vertebrate Skeleton. Cambridge: Cambridge University Press.

Nafei A, Danielsen CC, Linde F, and Hvid I. 2000a. Properties of growing trabecular ovine bone, part I: mechanical and physical properties. Journal of Bone and Joint Surgery 82B:910-920.

Nafei A, Kabel J, Odgaard A, Linde F, and Hvid I. 2000b. Properties of growing trabecular ovine bone, part II: architectural and mechanical properties. Journal of Bone and Joint Surgery 82B:921-927. 
2582

2583

2584

2585

2586

2587

2588

2589

2590

2591

2592

2593

2594

2595

2596

2597

2598

2599

2600

2601

2602

2603

2604

2605

2606

2607

2608

2609

2610

2611

2612

2613

2614

2615

2616

2617

2618

2619

2620

2621

2622

2623

2624

2625

Naish D. 2012. Birds. In: Brett-Surman MK, Holtz TR, Jr, and Farlow JO, eds. The Complete Dinosaur. 2 ed. Bloomington: Indiana University Press, 379-423.

Novas FE. 1996. Dinosaur monophyly. Journal of Vertebrate Paleontology 16:723-741.

Odgaard A. 1997. Three-Dimensional Methods for Quantification of Cancellous Bone Architecture. Bone 20:315-328.

Odgaard A. 2001. Quantification of Cancellous Bone Architecture. In: Cowin SC, ed. Bone Biomechanics Handbook. 2 ed. Boca Raton: CRC Press, 14-11-14-19.

Odgaard A, Kabel J, van Rietbergen B, Dalstra M, and Huiskes R. 1997. Fabric and elastic principal directions of cancellous bone are closely related. Journal of Biomechanics 30:487-495.

Ollion J, Cochennec J, Loll F, Escudé C, and Boudier T. 2013. TANGO: A Generic Tool for High-throughput 3D Image Analysis for Studying Nuclear Organization. Bioinformatics 29:1840-1841.

Olmos M, Casinos A, and Cubo J. 1996. Limb allometry in birds. Annales des Sciences naturelles, Zoologie, Paris 17:39-49.

Ostrom JH. 1969. Osteology of Deinonychus antirrhopus, and unusual theropod from the Lower Cretaceous of Montana. Bulletin of the Peabody Museum of Natural History 30:1-165.

Owen R. 1866. On the Osteology of the Dodo (Didus ineptus, Linn.). Transactions of the Zoological Society of London 5:49-85.

Oxnard CE, and Yang HC. 1981. Beyond biometrics: studies of complex biological patterns. Symposium of the Zoological Society of London 46:127.

Parfitt AM. 1983. The physiologic and clinic significance of bone histomorphometric data. In: Recker RR, ed. Bone Histomorphometry: Techniques and Interpretation. Boca Raton: CRC Press, 143-223.

Paul GS. 1988. Predatory Dinosaurs of the World. New York: Simon \& Schuster.

Paul GS. 1998. Limb design, function and running performance in ostrich-mimics and tyrannosaurs. Gaia 15:257-270.

Paul GS. 2008. The Extreme Lifestyles and Habits of the Gigantic Tyrannosaurid Superpredators of the Late Cretaceous of North America and Asia. In: Larsen PL, and Carpenter K, eds. Tyrannosaurus rex, the Tyrant King. Bloomington: Indiana University Press, 307-354.

Pauwels F. 1980. Biomechanics of the Locomotor Apparatus. Berlin: Springer-Verlag.

Pearson OM, and Lieberman DE. 2004. The Aging of Wolff's "Law": Ontogeny and Responses to Mechanical Loading in Cortical Bone. Yearbook of Physical Anthropology 47:63-99.

Petterson U, Nilsson M, Sundh V, Mellström D, and Lorentzon M. 2010. Physical activity is the strongest predictor of calcaneal peak bone mass in young Swedish men. Osteoporosis International 21:447-455.

Phillips ATM. 2012. Structural optimisation: biomechanics of the femur. Proceedings of the Institution of Civil Engineers - Engineering and Computational Mechanics 165:147-154.

Phillips ATM, Villette CC, and Modenese L. 2015. Femoral bone mesoscale structural architecture prediction using musculoskeletal and finite element modelling. International Biomechanics 2:43-61.

Phillips MJ, Gibb GC, Crimp EA, and Penny D. 2010. Tinamous and Moa Flock Together: Mitochondrial Genome Sequence Analysis Reveals Independent Losses of Flight among Ratites. Systematic Biology 59:90-107. 
2626

2627

2628

2629

2630

2631

2632

2633

2634

2635

2636

2637

2638

2639

2640

2641

2642

2643

2644

2645

2646

2647

2648

2649

2650

2651

2652

2653

2654

2655

2656

2657

2658

2659

2660

2661

2662

2663

2664

2665

2666

2667

2668

2669

2670

Pidaparti RMV, and Turner CH. 1997. Cancellous bone architecture: advantages of nonorthogonal trabecular alignment under multidirectional joint loading. Journal of Biomechanics 30:979-983.

Pittman M, Gatesy SM, Upchurch P, Goswani A, and Hutchinson JR. 2013. Shake a Tail Feather: The Evolution of the Theropod Tail into a Stiff Aerodynamic Surface. PLoS ONE 8:e63115.

Podsiadlo P, Dahl L, Englund M, Lohmander LS, and Stachowiak GW. 2008. Differences in trabecular bone texture between knees with and without radiographic osteoarthritis detected by fractal methods. Osteoarthritis and Cartilage 16:323-329.

Polk JD, Blumenfeld J, and Ahluwalia D. 2008. Knee Posture Predicted From Subchondral Apparent Density in the Distal Femur: An Experimental Validation. The Anatomical Record 16:323-329.

Pontzer H, Allen V, and Hutchinson JR. 2009. Biomechanics of Running Indicates Endothermy in Bipedal Dinosaurs. PLoS ONE 4:e7783.

Pontzer H, Lieberman DE, Momin E, Devlin MJ, Polk JD, Hallgrímsson B, and Cooper DML. 2006. Trabecular bone in the bird knee responds with high sensitivity to changes in load orientation. Journal of Experimental Biology 209:57-65.

Prum RO, Berv JS, Dornburg A, Field DJ, Townsend JP, lemmon EM, and Lemmon AR. 2015. A comprehensive phylogeny of birds (Aves) using targeted next-generation DNA sequencing. Nature 526:569-573.

Radin EL, Orr RB, Kelman JL, Paul IL, and Rose RM. 1982. Effect of prolonged walking on concrete on the knees of sheep. Journal of Biomechanics 15:487-492.

Rafferty KL. 1998. Structural design of the femoral neck in primates. Journal of Human Evolution 34:361-383.

Raichlen DA, Gordon AD, Foster AD, Webber JT, Sukhdeo SM, Scott RS, Gosman JH, and Ryan TM. 2015. An ontogenetic framework linking locomotion and trabecular bone architecture with applications for reconstructing hominin life history. Journal of Human Evolution 81:1-12.

Reid REH. 2012. "Intermediate” Dinosaurs: The Case Updated. In: Brett-Surman MK, Holtz TR, Jr, and Farlow JO, eds. The Complete Dinosaur. 2 ed. Bloomington: Indiana University Press, 873-921.

Reilly SM. 2000. Locomotion in the Quail (Coturnix japonica): The Kinematics of Walking and Increasing Speed. Journal of Morphology 243:173-185.

Robling AG, Castillo AB, and Turner CH. 2006. Biomechanical and Molecular Regulation of Bone Remodeling. Annual Review of Biomedical Engineering 8:455-498.

Robling AG, and Turner CH. 2009. Mechanical Signaling for Bone Modeling and Remodeling. Critical Reviews in Eukaryote Gene Expression 19:319-338.

Rook L, Bondioli L, Köhler M, Moyà-Solà S, and Macchiarelli R. 1999. Oreopithecus was a bipedal ape after all: Evidence from the iliac cancellous architecture. Proceedings of the National Academy of Sciences 96:8795-8799.

Rubenson J, Lloyd DG, Besier TF, Heliams DB, and Fournier PA. 2007. Running in ostriches (Stuthio camelus): three-dimensional joint axes alignment and joint kinematics. Journal of Experimental Biology 210:2548-2562.

Rudman KE, Aspden RM, and Meakin JR. 2006. Compression or tension? The stress distribution in the proximal femur. BioMedical Engineering OnLine 5:12. 
2671

2672

2673

2674

2675

2676

2677

2678

2679

2680

2681

2682

2683

2684

2685

2686

2687

2688

2689

2690

2691

2692

2693

2694

2695

2696

2697

2698

2699

2700

2701

2702

2703

2704

2705

2706

2707

2708

2709

2710

2711

2712

2713

2714

2715

2716
Ruimerman R, Hilbers P, van Rietbergen B, and Huiskes R. 2005. A theoretical framework for strain-related trabecular bone maintenance and adaptation. Journal of Biomechanics 38:931-941.

Russell DA. 1972. Ostrich Dinosaurs from the Late Cretaceous of Western Canada. Canadian Journal of Earth Sciences 9:375-402.

Ryan TM, and Ketcham RA. 2002a. Femoral head trabecular bone structure in two omomyid primates. Journal of Human Evolution 43:241-263.

Ryan TM, and Ketcham RA. 2002b. The three-dimensional structure of trabecular bone in the femoral head of strepsirrhine primates. Journal of Human Evolution 43:1-26.

Ryan TM, and Ketcham RA. 2005. Angular Orientation of Trabecular Bone in the Femoral Head and Its Relationship to Hip Joint Loads in Leaping Primates. Journal of Morphology 265:249-263.

Ryan TM, and Krovitz GE. 2006. Trabecular bone ontogeny in the human proximal femur. Journal of Human Evolution 51:591-602.

Ryan TM, and Shaw CN. 2012. Unique Suites of Trabecular Bone Features Characterize Locomotor Behavior in Human and Non-Human Anthropoid Primates. PLoS ONE 7:e41037.

Ryan TM, and Shaw CN. 2013. Trabecular bone microstructure scales allometrically in the primate humerus and femur. Proceedings of the Royal Society of London, Series B 280:20130172.

Ryan TM, and Test M. 2007. Methodological Issues in Comparative Analyses of Trabecular Bone Morphology. Eighth International Conference of Vertebrate Morphology, July 1621, 2007. Paris, France. p 1126-1127.

Ryan TM, and van Rietbergen B. 2005. Mechanical Significance of Femoral head Trabecular Bone structure in Loris and Galago Evaluated Using Micromechanical Finite Element Models. American Journal of Physical Anthropology 126:82-96.

Saers JPP, Cazorla-Bak Y, Shaw CN, Stock JT, and Ryan TM. 2016. Trabecular bone structural variation throughout the human lower limb. Journal of Human Evolution 97:97-108.

Saparin P, Scherf H, Hublin J-J, Fratzl P, and Weinkamer R. 2011. Structural Adaptation of Trabecular Bone Revealed by Position Resolved Analysis of Proximal Femora of Different Primates. The Anatomical Record 294:55-67.

Scherf H. 2008. Locomotion-related Femoral Trabecular Architectures in Primates - High Resolution Computed Tomographies and Their Implications for Estimations of Locomotor Preferences of Fossil Primates. In: Endo H, and Frey R, eds. Anatomical Imaging: Towards a New Morphology. Tokyo: Springer, 39-59.

Scherf H, Harvati K, and Hublin J-J. 2013. A comparison of proximal humeral cancellous bone of great apes and humans. Journal of Human Evolution 65:29-38.

Schulte FA, Ruffoni D, Lambers FM, Christen D, Webster DJ, Kuhn G, and Müller R. 2013. Local mechanical stimuli regulate bone formation and resorption in mice at the tissue level. PLoS ONE 8:e62172.

Schweitzer MH, Wittmeyer JL, and Horner JR. 2005. Gender-Specific Reproductive Tissue in Ratites and Tyrannosaurus rex. Science 308:1456-1460.

Sereno PC. 1999. The evolution of dinosaurs. Science 284:2137-2147.

Shrout PE, and Fleiss JL. 1979. Intraclass Correlations: Uses in Assessing Rater Reliability. Psychological Bulletin 86:420-428.

Singh I. 1978. The architecture of cancellous bone. Journal of Anatomy 127:305-310. 
2717 Singh M, Nagrath AR, and Maini PS. 1970. Changes in trabecular pattern of the upper end of the

2718

2719

2720

2721

2722

2723

2724

2725

2726

2727

2728

2729

2730

2731

2732

2733

2734

2735

2736

2737

2738

2739

2740

2741

2742

2743

2744

2745

2746

2747

2748

2749

2750

2751

2752

2753

2754

2755

2756

2757

2758

2759

2760

2761 femur as an index of osteoporosis. Journal of Bone and Joint Surgery 52B:457-467.

Skedros JG, and Baucom SL. 2007. Mathematical analysis of trabecular 'trajectories' in apparent trajectorial structures: The unfortunate historical emphasis on the human proximal femur. Journal of Theoretical Biology 244:15-45.

Skedros JG, Hunt KJ, and Bloebaum RD. 2004. Relationships of Loading History and Structural and Material Characteristics of Bone: Development of the Mule Deer Calcaneus. Journal of Morphology 259:281-307.

Skedros JG, Sorenson SM, Hunt KJ, and Holyoak JD. 2007. Ontogenetic Structural and Material Variations in Ovine Calcanei: A Model for Interpreting Bone Adaptation. The Anatomical Record 290:284-300.

Skerry T. 2000. Biomechanical influences on skeletal growth and development. In: O'Higgins P, and Cohn MJ, eds. Development, Growth, and Evolution: Implications for the Study of the Hominid Skeleton. San Diego: Academic Press, 29-39.

Skinner MM, Stephens NB, Tsegai ZJ, Foote AC, Nguyen NH, Gross T, Pahr DH, Hublin J-J, and Kivell TL. 2015. Human-like hand use in Australopithecus africanus. Science 347:395-399.

Smith JV, Braun EL, and Kimball RT. 2013. Ratite Nonmonophyly: Independent Evidence from 40 Novel Loci. Systematic Biology 62:35-49.

Smith NC, Jespers KJ, and Wilson AM. 2010. Ontogenetic scaling of locomotor kinetics and kinematics of the ostrich (Struthio camelus). Journal of Experimental Biology 213:13471355.

Smith TS, Martin RB, Hubbard M, and Bay BK. 1997. Surface Remodeing of Trabecular Bone Using a Tissue Level Model. Journal of Orthopaedic Research 15:593-600.

Stephens NB, Kivell TL, Gross T, Pahr DH, Lazenby RA, Hublin J-J, Hershkovitz I, and Skinner MM. 2016. Trabecular architecture in the thumb of Pan and Homo: implications for investigating hand use, loading, and hand preference in the fossil record. American Journal of Physical Anthropology 161:603-619.

Stoessel A, and Fischer MS. 2012. Comparative intralimb coordination in avian bipedal locomotion. Journal of Experimental Biology 215:4055-4069.

$\mathrm{Su}$ A, and Carlson KJ. 2017. Comparative analysis of trabecular bone structure and orientation in South African hominin tali. Journal of Human Evolution 106:1-18.

Su A, Wallace IJ, and Nakatsukasa M. 2013. Trabecular bone anisotropy and orientation in an Early Pleistocene hominin talus from East Turkana, Kenya. Journal of Human Evolution 64:667-677.

Su SC, Skedros JG, Bachus KN, and Bloebaum RD. 1999. Loading conditions and cortical bone construction of an artiodactyl calcaneus. Journal of Experimental Biology 202:32393254.

Sues H-D. 1978. Functional morphology of the dome in pachycephalosaurid dinosaurs. Neus Jahrbuch fur Geologie und Palaontologie 1978:459-472.

Sutherland D. 1997. The development of mature gait. Gait \& Posture 6:163-170.

Sverdlova N. 2011. Tensile trabeculae - Myth or Reality? Journal of Musculoskeletal and Neuronal Interactions 11:1-7.

Swartz SM, Parker A, and Huo C. 1998. Theoretical and empirical scaling patterns and topological homology in bone trabeculae. Journal of Experimental Biology 201:573-590. 
2762

2763

2764

2765

2766

2767

2768

2769

2770

2771

2772

2773

2774

2775

2776

2777

2778

2779

2780

2781

2782

2783

2784

2785

2786

2787

2788

2789

2790

2791

2792

2793

2794

2795

2796

2797

2798

2799

2800

2801

2802

2803

2804

2805
Takechi H. 1977. Trabecular architecture of the knee joint. Acta Orthopaedica Scandinavica 48:673-681.

Tanck E, Homminga J, van Lenthe GH, and Huiskes R. 2001. Increase in Bone Volume Fraction Precedes Architectural Adaptation in Growing Bone. Bone 28:650-654.

Thomas DA, and Farlow JO. 1997. Tracking a Dinosaur Attack. Scientific American 277:74-79.

Thomason JJ. 1985. The relationship of trabecular architecture to inferred loading patterns in the third metacarpals of the extinct equids Merychippus and Mesohippus. Paleobiology 11:323-335.

Thomason JJ. 1995. To what extent can the mechanical environment of a bone be inferred from its internal architecture? In: Thomason JJ, ed. Functional Morphology in Vertebrate Paleontology. New York: Cambridge University Press, 249-263.

Thompson DW. 1942. On Growth and Form. Cambridge: Cambridge University Press.

Thulborn RA. 1984. Preferred gaits of bipedal dinosaurs. Alcheringa 8:243-252.

Thulborn T. 1990. Dinosaur Tracks. London: Chapman and Hall.

Tobin WJ. 1955. The internal architecture of the femur and its clinical significance: the upper end. Journal of Bone and Joint Surgery 37A:57-72.

Townsley W. 1944. The architectural structure of the upper end of the femur in various pathological conditions. Journal of Pathology 56:199-207.

Townsley W. 1948. The influence of mechanical factors on the development and structure of bone. American Journal of Physical Anthropology 6:25-46.

Tsai HP, and Holliday CM. 2015. Articular Soft Tissue Anatomy of the Archosaur Hip Joint: Structural Homology and Functional Implications. Journal of Morphology 276:601-630.

Tsai HP, Middleton KM, Hutchinson JR, and Holliday CM. 2018. Hip joint articular soft tissues of non-dinosaurian Dinosauromorpha and early Dinosauria: evolutionary and biomechanical implications for Saurischia. Journal of Vertebrate Paleontology 38:e1427593.

Tsegai ZJ, Kivell TL, Gross T, Nguyen NH, Pahr DH, Smaers JB, and Skinner MM. 2013. Trabecular Bone Structure Correlates with Hand Posture and Use in Hominoids. PLoS ONE 8:e78781.

Tsegai ZJ, Skinner MM, Gee AH, Pahr DH, Treece GM, Hublin J-J, and Kivell TL. 2017. Trabecular and cortical bone structure of the talus and distal tibia in Pan and Homo. American Journal of Physical Anthropology 163:784-805.

Tsubota K, Adachi T, and Tomita Y. 2002. Functional adaptation of cancellous bone in human proximal femur predicted by trabecular surface remodeling simulation toward uniform stress state. Journal of Biomechanics 35:1541-1551.

Tsubota K, Suzuki Y, Yamada T, Hojo M, Makinouchi A, and Adachi T. 2009. Computer simulation of trabecular remodelling in human proximal femur using large-scale voxel FE models: Approach to understanding Wolff's law. Journal of Biomechanics 42:10881094.

Turner AH, Makovicky PJ, and Norell MA. 2012. A review of dromaeosaurid systematics and paravian phylogeny. Bulletin of the American Museum of Natural History 371:1-206.

Turner AH, Pol D, Clarke JA, Erickson GM, and Norell MA. 2007. A Basal Dromaeosaurid and Size Evolution Preceding Avian Flight. Science 317:1378-1381.

Turner CH. 1992. On Wolff's Law of trabecular architecture. Journal of Biomechanics 25:1-9. 
2806

2807

2808

2809

2810

2811

2812

2813

2814

2815

2816

2817

2818

2819

2820

2821

2822

2823

2824

2825

2826

2827

2828

2829

2830

2831

2832

2833

2834

2835

2836

2837

2838

2839

2840

2841

2842

2843

2844

2845

2846

2847

2848

2849

2850

Turner CH, Anne V, and Pidaparti RMV. 1997. A uniform strain criterion for trabecular bone adaptation: do continuum-level strain gradients drive adaptation? Journal of Biomechanics 30:555-563.

Turner CH, Cowin SC, Rho JY, Ashman RB, and Rice JC. 1990. The fabric dependence of the orthotropic elastic constants of cancellous bone. Journal of Biomechanics 23:549-561.

Ulrich D, van Rietbergen B, Laib A, and Rüegsegger P. 1999. The Ability of Three-Dimensional Structural Indices to Reflect Mechanical Aspects of Trabecular Bone. Bone 25:55-60.

van der Meulen MCH, Morgan TG, Yang X, Baldini TH, Myers ER, Wright TM, and Bostrom MPG. 2006. Cancellous bone adaptation to in vivo loading in a rabbit model. Bone 38:871-877.

van der Reest AJ, and Currie PJ. 2017. Troodontids (Theropoda) from the Dinosaur Park Formation, Alberta, with a description of a unique new taxon: implications for deinonychosaur diversity in North America. Canadian Journal of Earth Sciences 54:919935.

van Rietbergen B, Huiskes R, Eckstein F, and Rüegsegger P. 2003. Trabecular Bone Tissue Strains in the Healthy and Osteoporotic Human Femur. Journal of Bone and Mineral Research 18:1781-1788.

van Rietbergen B, Müller R, Ulrich D, Rüegsegger P, and Huiskes R. 1999. Tissue stresses and strain in trabeculae of a canine proximal femur can be quantified from computer simulations. Journal of Biomechanics 32:165-173.

Vander Sloten J, and Van der Perre G. 1989. Trabecular structure compared to stress trajectories in the proximal femur and calcaneus. Journal of Biomedical Engineering 11:203-208.

Verner KA, Lehner M, Lamas LP, and Main RP. 2016. Experimental tests of planar strain theory for predicting cross-sectional longitudinal and shear strains. Journal of Experimental Biology 219:3082-3090.

Volpato V. 2008. Morphogenèse de l'endostructure osseuse de l'ilion humain. Comptes Rendus Palevol 7:463-471.

von Meyer GH. 1867. Die Architektur der Spongiosa. Archiv für Anatomie, Physiologie und Wissenschaftliche Medicin 34:615-628.

Wainwright SA, Biggs WD, Currey JD, and Gosline JM. 1976. Mechanical Design in Organisms. London: Edward Arnold (Publishers) Limited.

Wallace IJ, Demes B, C. M, Pearson OM, Polk JD, and Lieberman DE. 2014. Exercise-Induced Bone Formation is Poorly Linked to Local Strain Magnitude in the Sheep Tibia. PLoS ONE 9:e99108.

Wallace IJ, Judex S, and Demes B. 2015. Effects of load-bearing exercise on skeletal structure andmechanics differ between outbred populations of mice. Bone 72:1-8.

Wallace IJ, Tommasini SM, Judex S, Garland T, Jr, and Demes B. 2012. Genetic Variations and Physical Activity as Determinants of Limb Bone Morphology: An Experimental Approach Using a Mouse Model. American Journal of Physical Anthropology 148:24-35.

Wang H, Ji B, Liu XS, Guo XE, Huang Y, and Hwang K-C. 2012. Analysis of microstructural and mechanical alterations of trabecular bone in a simulated three-dimensional remodelling process. Journal of Biomechanics 45:2417-2425.

Ward FO. 1838. Outlines of Human Osteology. London: Henry Renshaw.

Warton DI, Wright IJ, Falster DS, and Westoby M. 2006. Bivariate line-fitting methods for allometry. Biological Reviews 81:259-291. 
2851

2852

2853

2854

2855

2856

2857

2858

2859

2860

2861

2862

2863

2864

2865

2866

2867

2868

2869

2870

2871

2872

2873

2874

2875

2876

2877

2878

2879

2880

2881

2882

2883

2884

2885

2886

2887

2888

2889

Weingarten VI, Seide P, and Petersen JP. 1968. Buckling of thin-walled circular cylinders. NASA Space Vehicle Design Criteria (Structures) Monographs SP-8007:1-49.

Weishampel DB, Dodson P, and Osmólska H. 2004. The Dinosauria. 2 ed. Berkeley: University of California Press.

Wellnhofer P. 1991. The Illustrated Encyclopedia of Pterosaurs. New York: Crescent Books.

Wilson JA, and Carrano MT. 1999. Titanosaurs and the origin of "wide-gauge" trackways: a biomechanical and systematic perspective on sauropod locomotion. Paleobiology 25:252267.

Winter DA. 2009. Biomechanics and Motor Control of Human Movement. Hoboken: John Wiley \& Sons, Inc.

Wolff J. 1892. Das Gesetz der Transformation der Knochen. Berlin: August Hirschwald.

Wolschrijn CF, and Weijs WA. 2005. Development of the Subchondral Bone Layer of the Medial Coronoid Process of the Canine Ulna. The Anatomical Record 284A:439-445.

Yang P-F, Sanno M, Ganse B, Koy T, Brüggemann G-P, Müller LP, and Rittweger J. 2014. Torsion and Antero-Posterior Bending in the In Vivo Human Tibia Loading Regimes during Walking and Running. PLoS ONE 9:e94525.

Yuri T, Kimball RT, Harshman J, Bowie RCK, Braun MJ, Chojnowski JL, Han K-L, Hackett SJ, Huddleston CJ, Moore WS, Reddy S, Sheldon FH, Steadman DW, Witt CC, and Braun EL. 2013. Parsimony and Model-Based Analyses of Indels in Avian Nuclear Genes Reveal Congruent and Incongruent Phylogenetic Signals. Biology 2:419-444.

Zanno LE, Gillette DD, Albright LB, and Titus AL. 2009. A new North American therizinosaurid and the role of herbivory in 'predatory' dinosaur evolution. Proceedings of the Royal Society of London, Series B 276:3505-3511.

\section{$\underline{\text { I.9 Figure captions }}$}

Figure 1. Cancellous bone occurrence and macrostructure, as illustrated here with the femur of a cow (Bos tauros), sectioned in the coronal plane. (A) Cancellous bone occurs in the proximal and distal ends of the bone (as indicated by the braces), underlying the thin cortical bone capping the epiphyses and apophyses, as well as the metaphyses. (B) A close-up view of the cancellous bone reveals the high porosity of the tissue, giving it a spongy appearance.

Figure 2. Cancellous bone fabric as represented by its principal architectural directions. (A) A cube of cancellous bone of side length $5.33 \mathrm{~mm}$, from the proximal femur of a freshwater crocodile, Crocodylus johnstoni, with the principal directions of the bone's architecture superimposed. As an orthotropic material, cancellous bone fabric is completely described by three principal directions. (B) Tthe fabric ellipsoid representation for this cube of cancellous bone is derived from the vectors that describe the principal architectural directions. The ellipsoid's 
2890 major, semimajor and minor axes are given by the primary $\left(\mathbf{u}_{1}\right)$, secondary $\left(\mathbf{u}_{2}\right)$ and tertiary $\left(\mathbf{u}_{3}\right)$

2891 directions of the cancellous bone architecture, which correspond to the eigenvectors of the fabric

2892 tensor. The relative lengths of each axis depend on the relative magnitudes of the principal

2893 directions, which correspond to the eigenvalues of the fabric tensor. The degree of anisotropy

2894 (DA) describes the extent to which the trabeculae are aligned within a sample, and is given as the 2895 relative magnitude of the primary and tertiary eigenvalues (i.e., DA $=e_{1} / e_{3}$ ); in this instance DA

$2896=1.44$. The cancellous bone geometry was derived via micro-computed X-ray tomographic 2897 scanning (Siemens Inveon, $80 \mathrm{kV}, 500 \mu \mathrm{A}, 900 \mathrm{~ms}$ exposure, $53.3 \mu \mathrm{m}$ isotropic resolution) and 2898 3-D visualization (Mimics 17.0, Materialise NV, Belgium). The material directions were 2899 calculated using the mean intercept length method as implemented in the software Quant3D 2.3 2900 (see Ketcham \& Ryan 2004).

2901

2902

2903

Figure 3. Cancellous bone fabric direction can change in response to experimentally induced 2904 changes in mechanical loading. (A-C) The study of Goldstein et al. (1991). In the distal femur of 2905 normal dogs (A), the principal directions of cancellous bone fabric (arrows) vary throughout the bone. After 38 weeks following surgical implantation of load cells (B, arrows indicate direction of applied principal compressive stress), the principal directions of the cancellous fabric were greatly altered, and were reoriented to align with the compressive stress applied by the load cells (C). (D-F) The study of Pontzer et al. (2006). Subjecting guineafowl to running on inclined treadmills caused them to move with a more flexed knee posture compared to running on the level (the angle $\theta$ is reduced). The postural change resulted in an altered relative orientation of the joint force that the distal femur experienced (E, F, red arrow), which after 45 days was found to produce a changed orientation of peak trabecular density (dotted arrow).

Figure 4. Trabeculae tend to align themselves with the orientation of principal stresses resulting from in vivo loading. (A) Coronal micro-computed tomographic section through a human proximal femur, illustrating the architecture of cancellous bone. Image provided courtesy of SCANCO Medical AG. (B) A typical loading regime experienced by the proximal femur during locomotion, here the single-legged stance phase of walking (after Rudman et al. 2006). This 
2921 consists of the joint reaction force applied by the acetabulum (JRF), the force of the adductor

2922 muscles pulling on the trochanter (add) and the small forces applied by the capsular ligaments 2923 (c). (C) Principal stress trajectories resulting from the loading regime in B, as calculated by a 2924 two-dimensional finite element analysis (after Rudman et al. 2006). Note the striking 2925 correspondence of the main tracts of trabeculae in A and the principal stress trajectories in C. 2926

Figure 5. Cancellous bone remodelling at the cellular level can bring about changes in the entire architecture at the whole-bone level. (A-D) Schematic illustration of the mechanostat of cancellous bone. Given an initial architecture in A, a change in the loading regime will lead to some parts becoming overloaded (high stress, dotted) and others becoming underloaded (low stress, horizontal hatching) in B. Surface remodelling by osteoblasts and osteoclasts (C) acts to deposit additional bone material in those overloaded areas (dark grey) and remove bone material from those underloaded areas (light grey); arrows show direction in which local bone surface moves. This continues ad infinitum until all bone tissue is neither too highly strained nor too little strained. (E, F) Illustrates the application of the mechanostat principal on the level of the whole bone, via computational modelling (adapted from Jang \& Kim 2008; Jang \& Kim 2010a). In this example of the human proximal femur, with loads simulating both the joint reaction force and forces from the abductor muscles, the initially isotropic architecture (E) undergoes remodelling until equilibrium is reached. The resulting equilibrium architecture $(F)$ is extremely similar to that observed in the real specimen (cf. Fig. 4A)

Figure 6. Orthogonal arrangements of trabeculae usually reflect a highly consistent loading regime experienced by a bone. (A) Sagittal section through the calcaneum of a cow, with the pull of the Achilles tendon on the distal end indicated by the arrow. (B) A force applied to the free end of a cantilever beam is comparable to the loading regime experienced by the cow calcaneum during locomotion. The bending of the cantilever beam produces principal stress trajectories that are very similar to the overall arrangement of trabeculae in the calcaneum (solid lines are trajectories of compressive stress; dashed lines are trajectories of tensile stress). Since the 
2951 calcaneum is only loaded in this fashion, the two systems of trabeculae (one curving from up, one 2952 curving down) tend to intersect at right angles.

2953

2954

2955

2956

Figure 7. Three different ways in which the architecture of cancellous bone can be influenced by its mechanical environment. See main text for full discussion. The dashed grey pathway for epigenetics signifies that it currently remains unknown as to if and how epigenetics may influence cancellous bone mechanobiology.

2959

2960

2961

Figure 8. Phylogenetic interrelationships of the major groups of animals studied.

2962 Interrelationships of non-avian theropods are based on Zanno et al. (2009), Carrano et al. (2012) and Turner et al. (2012); interrelationships of birds are based on Hackett et al. (2008), MorganRichards et al. (2008), Ksepka (2009), Phillips et al. (2010), Haddrath \& Baker (2012), Jetz et al. (2012), Smith et al. (2013), Yuri et al. (2013), Mitchell et al. (2014), Jarvis et al. (2014), Ksepka $\&$ Phillips (2015) and Prum et al. (2015). The interrelationships of the neoavian species studied here are currently not well agreed upon, and so Neoaves is shown as an unresolved polytomy. Silhouettes depict exemplar members or each group, and are not to scale. The individual determinate species of non-avian theropod studied are listed in the inset box, and their phylogenetic position signified by superscripts.

2972

Figure 9. Resampling the medical CT scans, as per protocol 2, helps produce a more accurate segmentation of the cancellous bone structure. This is illustrated here with a CT slice of the proximal femur of a southern cassowary, Casuarius casuarius (QMO 31137). (A) The raw CT slice. (B) A local segmentation algorithm, as per protocol 1, is applied to the slice in A, with window radius 8 pixels and contrast threshold of 25; notice that it performs poorly, with some trabeculae becoming disconnected and some intertrabecular spaces being obliterated. (C) The same CT slice as in A, but resampled to triple the resolution, that is, voxel dimensions are now $1 / 3$ of original in-plane pixel resolution. (D) A local segmentation is applied to the resampled slice in $\mathrm{C}$, using the same parameters as in B; notice that it performs far better in extracting the 
2982 cancellous structure. Bicubic interpolation results in smoother boundaries to the segmented scans, 2983 in contrast to lower-order (e.g., bilinear) interpolation. The location of the insets in each panel is 2984 shown in C.

2985

2986

2987 Figure 10. Schematic illustration of the image processing protocol used for the non-avian 2988 theropod micro-CT scans. (A) The original image, affected by both high and low frequency 2989 noise; segmentation of this image by global or local thresholding techniques will not work. (B) A 2990 low-radius median filter is applied to remove high-frequency noise. (C) A large-radius median filter is applied to isolate the low-frequency (background) noise. (D) The low-frequency filtered image in $\mathrm{C}$ is subtracted from the high-frequency filtered image in B. (E) A low-radius mean 2993 filter is applied, followed by a global high-pass segmentation to produce the final image.

Figure 11. The arrangement of VOIs for quantitative analysis of cancellous bone architecture 2997 follows the geometric pattern of cubic close-packed spheres. This is illustrated here for the proximal right femur of Troodontidae sp. (MOR 748). Each spherical VOI has a diameter of 5 $\mathrm{mm}$. In this arrangement, each VOI is just touching its neighbouring VOIs, and each sphere can be in contact with up to 12 other VOIs. (A, B) Shown in medial view. (C, D) Shown in oblique anteromedial view. Notice in $\mathrm{C}$ and $\mathrm{D}$ that VOI placement avoids the medullary cavity (orange) and cracks (green).

Figure 12. Definition of the anatomical coordinate system for the femur used in this study. This is illustrated here with the right femur of a Australian bustard, Ardeotis australis (MVB 20408), in anterior (A) and oblique anteromedial (B) views. The anatomical coordinate system is defined by the principal axis of inertia of the bone (dashed line) and the centres of two spheres fit to the distal condyles (white circles). For left femora, their geometry and calculated mean orientation of the primary fabric vector were mirrored prior to construction of an anatomical coordinate system. 
3013 Figure 13. Schematic illustration of two of the features of diaphyseal cancellous bone that were 3014 subject to categorical scoring. (A) The orientation of trabeculae with respect to the long axis of 3015 the shaft. (B) The tendency of trabeculae to be closely associated with other trabeculae. Upper 3016 row is oblique view, lower row is section-on view. These illustrations were given to the scorers to 3017 provide them with a point of reference.

3018

3019

3020

Figure 14. Pneumatization modulates trabecular spacing in the femur of both large and small 3021 birds. This is illustrated here with processed CT scan slices located approximately midway 3022 through the femoral head in the axial plane. (A) A marrow-filled femur of a southern cassowary, Casuarius casuarius (QMO 30105), with mean trabecular spacing $=0.638 \mathrm{~mm}$. (B) A pneumatized femur of an emu, Dromaius novaehollandiae (QMO 16140) with mean trabecular spacing $=1.128 \mathrm{~mm}$. (C) A marrow-filled femur of a chicken, Gallus gallus (PJB coll.), with mean trabecular spacing $=0.320 \mathrm{~mm}$. (D) A pneumatized femur of an Australian brush turkey, Alectura lathami (PJB coll.), with mean trabecular spacing $=0.999 \mathrm{~mm}$. Reported trabecular spacing values were calculated (for illustrative purposes) for the femoral head using the BoneJ 1.3.11 plugin for ImageJ (Doube et al. 2010). A and B are shown at the same scale, as are C and D. Scale bars are $10 \mathrm{~mm}$, and yellow asterisks denote pneumatopores.

Figure 15. The main architectural features of cancellous bone in the human proximal femur. (A) Vector field of $\mathbf{u}_{1}$ in the head, inferior neck and greater trochanter regions, plotted on a translucent rendering of the external bone geometry; view is in the coronal plane. Schematic inset illustrates close correspondence with the principal compressive (PC) and greater trochanter (GT) groups of previous studies. (B) Vector field of $\mathbf{u}_{1}$ (red) and $\mathbf{u}_{2}$ (blue) in the middle of the metaphysis, viewed in the coronal plane. Schematic inset illustrates close correspondence with the secondary compressive (SC) and secondary tensile (ST) groups of previous studies. Note that both $\mathbf{u}_{1}$ and $\mathbf{u}_{2}$ are largely parallel to the coronal plane. (C) Vector field of $\mathbf{u}_{1}$ in the distal metaphysis and lesser trochanter (in oblique proximomedial view), which is largely parallel to the bone's long-axis. In this and all subsequent illustrations of fabric vector fields, the length of each 
3043 fabric vector is proportional to its corresponding fabric eigenvalue. Additionally, all images are 3044 of bones from the right side of the body.

3045

3046

3047 Figure 16. The main architectural features of cancellous bone in the proximal femur of birds. (A3048 D) Vector field of $\mathbf{u}_{1}$ in the femoral head and inferior neck of an emu, Dromaius novaehollandiae (QMO 16140; A, B), and ostrich, Struthio camelus (MV R.2385; C, D). (E-H) Vector field of $\mathbf{u}_{1}$ under the facies antitrochanterica of a greater rhea, Rhea americana (QMO 23517; E, F), and chicken, Gallus gallus (PJB coll.; G, H). (I) Isosurface rendering of cancellous bone under the facies antitrochanterica of a dusky moorhen, Gallinula tenebrosa (PJB coll., between arrows), sectioned in the sagittal plane. ( $\mathrm{J}-\mathrm{M})$ Vector field of $\mathbf{u}_{1}$ in the trochanteric crest of a southern cassowary, Casuarius casuarius (QMO 30105; J, K), and Struthio camelus (MV R.2385, L, M). (N) Isosurface rendering of cancellous bone in the trochanteric crest of a magpie goose, Anseranus semipalmata (QMO 29529, between arrows), sectioned in the sagittal plane. (O, P) Vector field of $\mathbf{u}_{1}$ throughout the entire proximal femur of Dromaius novaehollandiae (QMO 16140, O) and Casuarius casuarius (QMO 30105, P), which illustrates the increasing obliquity and disorganization of vectors in the distal metaphysis and transition to the diaphysis, shown in regions with braces. A, C, J and L are anterior views; B and D are medial views; E and G are posterior views; $\mathrm{F}, \mathrm{H}, \mathrm{K}$ and $\mathrm{M}$ are lateral views; $\mathrm{O}$ is an oblique anterolateral view; $\mathrm{P}$ is an oblique anteromedial view. For reference, silhouettes of the animals depicted are provided in this figure and those that follow.

3064

3065

Figure 17. The main architectural features of cancellous bone in the proximal femur of extant sprawling reptiles. (A, B) Vector field of $\mathbf{u}_{1}$ in the proximal femur of a freshwater crocodile, Crocodylus johnstoni (QMJ 47916). (C, D) Vector field of $\mathbf{u}_{1}$ in the proximal femur of a Spencer's goanna, Varanus spenceri (QMJ 84416). (E) Vector field of $\mathbf{u}_{1}$ throughout the proximal femur of a Komodo dragon, Varanus komodoensis (AM R.106933), which illustrates the increasing obliquity and disorganization of vectors in the distal metaphysis and transition to the diaphysis, shown in region with braces. A and C are anterior views ('dorsal view' of 3073 herpetologists); B and D are lateral views ('posterior view' of herpetologists); E is an oblique 
3074 anterolateral view. For clarity, the vectors of $\mathbf{u}_{1}$ in the fourth trochanter are not visible in $\mathrm{A}, \mathrm{C}$ 3075 and E.

3076

3077

3078 Figure 18. The orientation of $\mathbf{u}_{1}$ throughout the femoral head of Masiakasaurus knopfleri, here 3079 exemplified by FMNH PR 2117. (A) Anterior view. (B) Lateral view.

Figure 19. The main architectural features of cancellous bone in the proximal femur of both Allosaurus and the tyrannosaurids. These are illustrated here with a 3-D geometric model of the observed architecture, mapped to the femur of Daspletosaurus torosus (TMP 2001.036.0001). (A-E) Five progressive rotations of the bone, in $30^{\circ}$ increments, from anteromedial to anterolateral views ( $\mathrm{C}$ is a purely anterior view). (F) The observed orientation of the dominant tract of cancellous bone in the femoral head (blue) has a gentle anterior inclination; bone shown in medial view. For explanation of the features and colour coding, refer to the main text. Inset below C is a CT slice through the proximal femur of Tyrannosaurus rex (MOR 1128), parallel to the coronal plane and through the middle of the femoral head. This illustrates the very characteristic tract of cancellous bone that extends from the base of the femoral neck up towards the apex of the head, highly comparable to the tract present in humans (cf. Fig. 4A).

Figure 20. The main architectural features of cancellous bone in the proximal femur of ornithomimids and caenagnathids. (A-D) Vector field of $\mathbf{u}_{1}(\mathrm{~A}, \mathrm{C})$ and $\mathbf{u}_{2}(\mathrm{~B}, \mathrm{D})$ in the femoral head and proximal metaphysis of an inteterminate ornithomimid (TMP 85.36.276), in oblique anteromedial (A, B) and oblique anterolateral (C, D) views. Note that the vector field along the anterior and posterior peripheries of the femoral head are not shown here (for clarity), where they are more typically oriented as in birds and humans. (E) Vector field of $\mathbf{u}_{1}$ in the greater trochanter region and distal metaphysis of an indeterminate ornithomimid (TMP 85.36.276), in oblique anterolateral view; note the increased obliquity and disorganization of vectors in the distal metaphysis (region with braces). (F) Vector field of $\mathbf{u}_{1}$ in the lesser trochanter of an indeterminate ornithomimid (TMP 91.36.569), in oblique anteromedial view. (G) Vector field of $\mathbf{u}_{1}$ in the 
3105 proximal femur of an indeterminate caenagnathid (TMP 86.36.323), in a 3-D slice parallel to the

3106 axial plane and through the femoral head and lesser trochanter. Main image is shown in axial

3107 view (anterior is toward bottom of page), with inset illustrating the region illustrated in context of

3108 the whole bone. The medialmost part of the femoral head is missing (dotted line).

Figure 21. The main architectural features of cancellous bone in the proximal femur of Falcarius utahensis and Troodontidae sp. (A) Vector field of $\mathbf{u}_{1}$ in the proximal femur of Falcarius (UMNH VP 12361), viewed as a 3-D slice parallel to the coronal plane and through the middle of the bone. Schematic inset illustrates three main trajectories in this image, which are not too dissimilar from the patterns observed in humans and large non-avian theropods (cf. Figs 15, 19). (B, C) Vector field of $\mathbf{u}_{1}(\mathrm{~B})$ and $\mathbf{u}_{2}(\mathrm{C})$ in the lesser trochanter of Falcarius, in oblique anterolateral view. (D) Vector field of $\mathbf{u}_{2}$ in the proximal femur of Falcarius, in a 3-D slice parallel to the axial plane and through the femoral head. Main image is shown in axial view (anterior is toward bottom of page), with inset illustrating the region illustrated in context of the whole bone. (E, F) Vector field of $\mathbf{u}_{1}$ in the femoral head and inferior neck of Troodontidae sp. (MOR 748) in anterior (E) and medial (F) views. (G, H) Vector field of $\mathbf{u}_{1}$ in the region of the greater trochanter of Troodontidae sp. (MOR 553s-7.28.91.239) in posterior $(\mathrm{G})$ and lateral $(\mathrm{H})$ views. (I) Orientation of $\mathbf{u}_{1}$ in the lesser trochanter, or immediate region thereof, of Troodontidae sp., in oblique anterolateral view (main image illustrates MOR 748; inset illustrates MOR 553s7.28.91.239). (J) Vector field of $\mathbf{u}_{1}$ throughout the metaphysis of Troodontidae sp. (MOR 553s7.28.91.239), illustrating increasing obliquity and disorganization of vectors in the distal metaphysis and transition to the diaphysis (region with braces).

Figure 22. The mean orientation of $\mathbf{u}_{1}$ in the femoral head, referenced in the femur anatomical coordinate system. This is plotted on an equal-angle stereoplot, with northern hemisphere projection (using StereoNet 9.5Allmendinger et al. 2013; Cardozo \& Allmendinger 2013). (A) Results for all specimens analysed. Note that for the fossil specimens, only those that were complete and well-preserved, and enabled an anatomical coordinate system to be defined, were analysed. Colour codes: black = birds, pink = human, blue = Masiakasaurus $($ FMNH PR 2153, 
3136 UA 8384), orange $=$ Falcarius, green $=$ Troodontidae sp. $($ MOR 748), purple $=$ general

3137 orientation for Allosaurus and the tyrannosaurids, red = mean orientation across birds. (B)

3138 Results for species or genera that were multiply sampled often displayed significant instraspecific 3139 or intrageneric variation, ranging up to $30.6^{\circ}$. Colour codes: black $=$ ostrich, Struthio camelus, 3140 blue $=$ southern cassowary, Casuarius casuarius, orange $=\mathrm{emu}$, Dromaius novaehollandiae, , red 3141 = chicken, Gallus gallus, green = kiwi, Apteryx spp. (C, D) The orientation of $\mathbf{u}_{1}$ superimposed 3142 on the CT scan of a representative tyrannosaur femur (Daspletosaurus torosus, TMP 31432001.036 .0001 ) in coronal (C) and sagittal (D) views, illustrating how the orientation data 3144 extracted and illustrated in the geometric models (e.g., Fig. 19) qualitatively reflects the observed 3145 cancellous bone architecture in the scans.

Figure 23. The main architectural features of cancellous bone in the human distal femur. (A) Vector field of $\mathbf{u}_{1}$ in a 3-D slice parallel to the coronal plane, made through the middle of the condyles. Schematic inset illustrates weakly developed double-arcuate pattern. (B) Vector field of $\mathbf{u}_{1}$ in a 3-D slice parallel to the sagittal plane, made between the condyles. Schematic inset illustrates weakly developed double-arcuate pattern. (C) Vector field of $\mathbf{u}_{1}$ in the medial condyle, shown for a 3-D slice parallel to the sagittal plane, made through the middle of the condyle. (D) Vector field of $\mathbf{u}_{2}$ at the level of the condyles, shown for a 3-D slice parallel to the axial plane, made through the middle of the condyles; anterior is toward top of page. Schematic inset illustrates the two distinctive tracts that comprise a 'butterfly pattern'.

Figure 24. The main architectural features of cancellous bone in the distal femur of birds. (A) Vector field of $\mathbf{u}_{1}$ in the central metaphysis of a southern cassowary, Casuarius casuarius (QMO 30105), in a 3-D slice, parallel to the sagittal plane and between the condyles, shown in lateral view. Note the weakly developed double arcuate pattern. (B, C) Vector field of $\mathbf{u}_{2}$ in a 3-D slice through the middle of the condyles in an ostrich, Struthio camelus (MV R.2385, B), and a emu, Dromaius novaehollandiae (QMO 11685, C), shown in distal view. Note the 'butterfly pattern' in both examples. (D) Isosurface rendering of cancellous bone in the distal condyles of a Japanese quail, Coturnix japonica (PJB coll.), sectioned in the axial plane; notice the 'butterfly pattern' 
3167 between the arrows. $(\mathrm{E}-\mathrm{H})$ Vector field of $\mathbf{u}_{1}$ in the medial condyle of Dromaius novaehollandiae 3168 (QMO 16140, E, F) and Casuarius casuarius (QMO 30604, G, H), shown in anterior (E, G) and 3169 medial (F, H) views. (I, J) Vector field of $\mathbf{u}_{1}$ in the lateral condyle of a chicken, Gallus gallus 3170 (PJB coll.), shown in anterior (I) and lateral (J) views. (K) Isosurface rendering of cancellous 3171 bone in the medial condyle of a purple swamphen, Porphyrio porphyrio (PJB coll.), sectioned in 3172 the sagittal plane. (L) Vector field of $\mathbf{u}_{1}$ throughout the entire distal femur of Casuarius casuarius 3173 (QMO 31137), illustrating increasing obliquity and disorganization of vectors in the proximal 3174 metaphysis and transition to the diaphysis (region with braces).

Figure 25. The main architectural features of cancellous bone in the distal femur of extant sprawling reptiles. (A, B) Vector field of $\mathbf{u}_{2}$ in a 3-D slice through the middle of the condyles in a freshwater crocodile, Crocodylus porosus (QMJ 48127, A), and a Komodo dragon, Varanus komodoensis (AM R.106933, B), shown in proximal view. (C, D) Vector field of $\mathbf{u}_{1}$ in the medial condyle of Crocodylus porosus (QMJ 48127), shown in anterior (C) and medial (D) views. (E, F) Vector field of $\mathbf{u}_{1}$ in the lateral condyle of a Spencer's goanna, Varanus spenceri (QMJ 484416), shown in anterior (E) and lateral (F) views.

Figure 26. The main architectural features of cancellous bone in the distal femur of both Allosaurus and the tyrannosaurids. These are illustrated here with a 3-D geometric model of the observed architecture, mapped to the femur of Daspletosaurus torosus (TMP 2001.036.0001). (A-G) Seven progressive rotations of the bone, in $30^{\circ}$ increments, from medial to lateral views ( $\mathrm{D}$ is a purely anterior view). Note that the architecture of the metaphysis was not observed in the Allosaurus specimens studied, owing to insufficient contrast or resolution in the CT scans failing to reveal any information about the metaphysis. For explanation of the features and colour

Figure 27. The main architectural features of cancellous bone in the distal femur of coding, refer to the main text. ornithomimids and caenagnathids. (A, B) Vector field of $\mathbf{u}_{1}$ in the lateral condyle of an 
3198 indeterminate ornithomimid (TMP 99.55.337) in posterior (A) and lateral (B) views. (C, D)

3199 Vector field of $\mathbf{u}_{1}$ in the medial condyle of an indeterminate caenagnathid (TMP 86.36.323) in 3200 posterior (C) and medial (D) views. (E, F) Vector field of $\mathbf{u}_{1}$ (E) and $\mathbf{u}_{2}(\mathrm{~F})$ in the distal femur of 3201 an indeterminate ornithomimid (TMP 91.36.569) at the level of the distal condyles, shown in 3202 proximal view for a 3-D slice parallel to the axial plane (inset shows location of slice). In A-D, 3203 the highlighted yellow vectors in the posterior extremities of the condyles have a much more 3204 mediolateral orientation compared to elsewhere in the condyle. This is also seen in E, where 3205 vectors that appear longer are more parallel to the axial plane, and vectors that appear shorter are 3206 more proximodistally oriented.

3207

Figure 28. The main architectural features of cancellous bone in the distal femur of Falcarius utahensis and Troodontidae sp. (A, B) Vector field of $\mathbf{u}_{1}$ in the medial condyle of Falcarius (UMNH VP 12360) in anterior (A) and medial (B) views. (C) Vector field of $\mathbf{u}_{1}$ throughout the distal femur of Troodontidae sp. (MOR 553s-7.28.91.239), illustrating increasing obliquity and disorganization of vectors in the proximal metaphysis and transition to the diaphysis (region with braces). (D, E) Vector field of $\mathbf{u}_{1}$ in the lateral condyle of Troodontidae sp. (MOR 748) in anterior (D) and lateral (E) views. (F) Vector field of $\mathbf{u}_{2}$ in the condyles of Troodontidae sp. (MOR 748), shown as a 3-D slice through the middle of the condyles in axial view; anterior is toward top of page.

Figure 29. The mean orientation of $\mathbf{u}_{1}$ in the medial femoral condyle, referenced in the femur anatomical coordinate system. This is plotted on an equal-angle stereoplot, with southern hemisphere projection (using StereoNet 9.5). (A) The results for all specimens analysed; for clarity, only the posteromedial quadrant of the plot is shown. Note that for the fossil specimens, only those that were complete and well-preserved, and enabled an anatomical coordinate system to be defined, were analysed. Colour codes: black = birds, pink = human, blue = Masiakasaurus $($ FMNH PR 2153, UA 8384), orange = Falcarius, green $=$ Troodontidae sp., purple = general orientation for Allosaurus and the tyrannosaurids, yellow = indeterminate ornithomimid (TMP 91.36.569), brown = indeterminate caenagnathid, red = mean orientation across birds. (B) 
3228 Comparison of posterior inclination of $\mathbf{u}_{1}$ in sagittal plane versus femur length in birds, with

3229 major axis regression line (and associated statistics) plotted.

3230

3231

3232

Figure 30. The main architectural feature of cancellous bone in the human proximal tibia is the gentle posterior inclination of $\mathbf{u}_{1}$ underneath the medial and lateral condyles. (A) Vector field of $\mathbf{u}_{1}$ under the lateral condyle, in lateral view. (B) Vector field of $\mathbf{u}_{1}$ under both condyles, in anterior view. (C) Vector field of $\mathbf{u}_{1}$ under the medial condyle, in medial view.

Figure 31. The main architectural features of cancellous bone in the proximal tibia of birds. (A, B) Vector field of $\mathbf{u}_{1}$ in the anterior (cranial) cnemial crest of an emu, Dromaius novaehollandiae (QMO 11686, A), and turkey, Meleagris gallopavo (RVC turkey 1, B), shown in medial view. (C, D) Vector field of $\mathbf{u}_{1}$ in the lateral cnemial crest of a southern cassowary, Casuarius casuarius (QMO 30105), shown in anterior (C) and lateral (D) views. (E) Isosurface rendering of cancellous bone in the anterior cnemial crest of an Australian white ibis, Threskiornis moluccus (PJB coll., between arrows), sectioned in the sagittal plane. (F) Isosurface rendering of cancellous bone in the lateral cnemial crest of a guineafowl, Numida meleagris (PJB coll., between arrows), sectioned in the plane of the crest. (G-J) Vector field of $\mathbf{u}_{1}$ under the medial condyle of an ostrich, Struthio camelus (MV R.2385, G, H), and chicken, Gallus gallus (PJB coll., I, J), shown in posterior $(\mathrm{G}, \mathrm{I})$ and medial $(\mathrm{H}, \mathrm{J})$ views. $(\mathrm{K})$ Isosurface rendering of cancellous bone under the medial condyle of an elegant-crested tinamou, Eudromia elegans (UMZC 404.e, between arrows), sectioned in the sagittal plane. ( $\mathrm{L}-\mathrm{O}$ ) Vector field of $\mathbf{u}_{1}$ under the lateral condyle of Struthio camelus (MV R.2711, L, M) and Dromaius novaehollandiae (QMO 11686, N, O), shown in posterior $(\mathrm{L}, \mathrm{N})$ and lateral $(\mathrm{M}, \mathrm{O})$ views. $(\mathrm{P})$ Isosurface rendering of cancellous bone under the lateral condyle of a little spotted kiwi, Apteryx owenii (UMZC 378.iii, between arrows), sectioned in the coronal plane. (Q) Vector field of $\mathbf{u}_{1}$ in a 3-D slice through the middle of the proximal metaphysis, cnemial crests and condyles of Dromaius novaehollandiae (QMO 11686), parallel to the sagittal plane. Schematic inset illustrates the moderately developed double-arcuate pattern present. (R) Vector field of $\mathbf{u}_{1}$ throughout the entire proximal tibia of Dromaius 
3258

3259

3260

3261

3262

3263

3264

3265

3266

3267

3268

3269

3270

3271

3272

3273

3274

3275

3276

3277

3278

3279

3280

3281

3282

3283

3284

3285

3286

3287

3288

novaehollandiae (QMO 11686), illustrating increasing obliquity and disorganization of vectors in the distal metaphysis and transition to the diaphysis (region with braces).

Figure 32. The main architectural features of cancellous bone in the proximal tibia of extant sprawling reptiles, as exemplified by a saltwater crocodile, Crocodylus porosus (QMJ 48127).

(A) Vector field of $\mathbf{u}_{1}$ in anterior view. (B) Vector field of $\mathbf{u}_{1}$ in medial view.

Figure 33. The main architectural features of cancellous bone in the proximal tibia of both Allosaurus and the tyrannosaurids. These are illustrated here with a 3-D geometric model of the observed architecture, mapped to the tibia of Daspletosaurus torosus (TMP 2001.036.0001). (A) The dominant orientation of cancellous bone in the medial condyle, in medial view. (B) The dominant orientation of cancellous bone in the medial and lateral condyles, in posterior view. (C) The dominant orientation of cancellous bone in the lateral condyle, in lateral view. (D-J) Seven progressive rotations of the bone, in $30^{\circ}$ increments, from proximally oblique medial to lateral views. For explanation of the features and colour coding, refer to the main text.

(1)

Figure 34. The main architectural features of cancellous bone in the proximal tibia of Troodontidae sp. and Saurornitholestes langstoni. (A, B) Vector field of $\mathbf{u}_{1}$ under the medial condyle of Troodontidae sp. (MOR 553s-7.11.91.41) in posterior (A) and medial (B) views. (C, D) Vector field of $\mathbf{u}_{1}$ under the lateral condyle of Troodontidae sp. (MOR 748) in posterior (C) and lateral (D) views. (E, F) Vector field of $\mathbf{u}_{1}$ in the cnemial crest of Troodontidae sp. (MOR 748) in lateral (E) and anterior (F) views. (G) Vector field of $\mathbf{u}_{1}$ in the cnemial crest of Saurornitholestes (MOR 660) in lateral view. (H) Vector field of $\mathbf{u}_{1}$ in a 3-D slice, parallel to the sagittal plane, through the central metaphysis of Troodontidae sp. (MOR 553s-7.28.91.239), shown in medial view. Schematic inset illustrates the moderately developed double-arcuate pattern present. 
3289 Figure 35. The main architectural features of cancellous bone in the human distal tibia. (A) 3290 Vector field of $\mathbf{u}_{1}$ in a 3-D slice, parallel to the coronal plane, through the middle of the bone, 3291 shown in anterior view. (B) Vector field of $\mathbf{u}_{1}$ in a 3-D slice, parallel to the sagittal plane, through 3292 the middle of the bone, shown in lateral view. (C) Vector field of $\mathbf{u}_{2}$ in a 3-D slice, parallel to the 3293 axial plane, through the distal end of the bone, shown in proximal view (anterior is toward top of 3294 page). Inset shows location of slice.

3295

3296

3297

Figure 36. The main architectural features of cancellous bone in the distal tibiotarsus of birds. (A-D) Vector field of $\mathbf{u}_{1}(\mathrm{~A}, \mathrm{C})$ and $\mathbf{u}_{2}(\mathrm{~B}, \mathrm{D})$ in the distal tibiotarsus of an emu, Dromaius novaehollandiae (QMO 16140), in oblique anterolateral (A, B) and oblique anteromedial (C, D) views. (E) Vector field of $\mathbf{u}_{1}$ (red) and $\mathbf{u}_{2}$ (blue) in the condyles of Dromaius novaehollandiae (QMO 16140) in proximal view (anterior is toward top of page). Note how both $\mathbf{u}_{1}$ and $\mathbf{u}_{2}$ are strongly aligned parallel to the sagittal plane. This particular specimen exemplifies a very stereotypical pattern that is observed in all large birds; the general pattern illustrated here was also observed in smaller species for which only limited fabric analysis was possible. (F) Isosurface rendering of cancellous bone in the distal tibiotarsus of a southern cassowary, Casuarius casuarius (QMO 30105), shown in oblique anteromedial view, with multiple cuts through the bone to illustrate 3-D architecture. (G) Isosurface rendering of cancellous bone in the distal tibiotarsus of a bustard, Ardeotis australis (MVB 20408), shown in oblique anterolateral view, with multiple cuts through the bone to illustrate 3-D architecture. $(\mathrm{H})$ Isosurface rendering of cancellous bone in the distal tibiotarsus of a painted quail, Coturnix chinensis (PJB coll.), sectioned in the axial plane through the middle of the condyles and shown in proximal view (anterior is toward top of page). In F and G, cut surfaces are coloured red to better show the nature of the cancellous bone architecture, in particular, the plate-like nature of many of the trabeculae, largely aligned parallel to the sagittal plane.

Figure 37. The main architectural features of cancellous bone in the distal tibia of extant sprawling reptiles. (A, B) Vector field of $\mathbf{u}_{1}$ in a Komodo dragon, Varanus komodoensis (AM 3319 R.106933) in anterior (A) and medial (B) views. (C) Vector field of $\mathbf{u}_{1}$ in a saltwater crocodile, 
3320

3321

3322

3323

3324

3325

3326

3327

3328

3329

3330

3331

3332

3333

3334

3335

3336

3337

3338

3339

3340

3341

3342

3343

3344

3345

3346

3347

3348

3349

3350

Crocodylus porosus (QMJ 48127) in anteromedial view. (D) Vector field of $\mathbf{u}_{2}$ in a 3-D slice through the distal end of the tibia of Crocodylus porosus (QMJ 48127), shown in proximal view (anterior is toward top of page). Inset shows location of slice.

Figure 38. The main architectural features of cancellous bone in the distal tibiotarsus of basal theropods. These are illustrated here with a 3-D geometric model of the observed architecture, mapped to the tibiotarsus of Daspletosaurus torosus (TMP 2001.036.0001; note that calcaneum was digitally sculpted based on other tyrannosaurid calcanei). (A-G) Seven progressive rotations of the tibia, in $30^{\circ}$ increments, from proximally oblique medial to lateral views. Schematic inset in D illustrates generic cross-sectional pattern at the level indicated. (H-J) Three views of the astragalus and calcaneum, corresponding to those in $\mathrm{C}-\mathrm{E}$, respectively. The various colours are used to help visualize the various tracts of cancellous bone more clearly.

Figure 39. The main architectural features of cancellous bone in the distal tibiotarsus of Troodontidae sp. and Saurornitholestes langstoni. (A, B) Vector field of $\mathbf{u}_{1}$ (red) and $\mathbf{u}_{2}$ (blue) in the distal tibiotarsus of Troodontidae sp. (MOR 748) in anterior (A) and proximal (B) views; in $\mathrm{B}$, anterior is toward top of page. Note how both $\mathbf{u}_{1}$ and $\mathbf{u}_{2}$ are generally aligned parallel to the sagittal plane. (C) Isosurface rendering of cancellous bone in the medial distal tibia of Saurornitholestes (MOR 660), shown in oblique anteromedial view, with multiple cuts through the bone (cut surfaces are coloured red) to illustrate 3-D architecture.

Figure 40. The main architectural features of cancellous bone in the fibula. (A, B) Vector field of $\mathbf{u}_{1}$ in the human fibula, in anterior (A) and lateral (B) views. (C, D) Vector field of $\mathbf{u}_{1}$ in a freshwater crocodile, Crocodylus johnstoni (QMJ 47916), in anterior (C) and lateral (D) views. (E, F) Vector field of $\mathbf{u}_{1}$ in an Argus monitor, Varanus panoptes (QMJ 91981), in anterior (E) and lateral (F) views. (G-I) Vector field of $\mathbf{u}_{1}$ in the fibular head of an emu, Dromaius novaehollandiae (QMO 11686, G), greater rhea, Rhea americana (QMO 23517, H), and chicken, Gallus gallus (PJB coll., I), in lateral view. (J, K) Isosurface rendering of cancellous bone in the 
3351 proximal fibula of a malleefowl, Leipoa ocellata (MVB 20194, J), and painted quail, Coturnix 3352 chinensis (PJB coll., K), sectioned in the plane of the head and shown in lateral view. (L, M) The 3353 dominant architectural direction of cancellous bone in the fibula of Allosaurus and 3354 tyrannosaurids, shown in anterior (L) and lateral (M) views. This is illustrated here with a 3-D 3355 geometric model of the observed architecture, mapped to the fibula of Daspletosaurus torosus 3356 (TMP 2001.036.0001). (N) Vector field of $\mathbf{u}_{1}$ in the proximal fibula of Troodontidae sp. (MOR $3357553 \mathrm{~s}-8.17 .92 .265)$, in lateral view.

3358

3359

3360

3361

Figure 41. Oblique trabeculae in the diaphyses of birds and reptiles. These are illustrated here with a number of examples demonstrating the variety of forms the individual trabeculae can assume. (A) Femoral mid-shaft of an emu, Dromaius novaehollandiae (QMO 16140). (B) Proximal femoral shaft of a turkey, Meleagris gallopavo (PJB coll.). (C) Distal femoral shaft of a 3364 malleefowl, Leipoa ocellata (MVB 20194). (D) Proximal femoral shaft of Dromaius novaehollandiae (QMO 11686). (E) Proximal femoral shaft of an Australian brush turkey, Alectura lathami (PJB coll.). (F) Proximal femoral shaft of an elegant-crested tinamou, Eudromia elegans (UMZC 404.e). (G) Femoral mid-shaft of an Argus monitor, Varanus panoptes (QMJ 91981). (H) Distal femoral shaft of a little tinamou, Crypturellus soui (MVB 23647). (I) Tibial mid-shaft of an ostrich, Struthio camelus (MV R.2385). In all figures, proximal is towards the top of the page.

Figure 42. Size-dependent variation in diaphyseal cancellous bone architecture in the femora and tibiae of birds. These plots compare the results of the categorical scoring of each bone against its length. (A, B) Feature 1 (extent of cancellous bone) in the femur (A) and tibia (B); a higher score indicates greater extent. (C, D) Feature 2 (average orientation of trabeculae) in the femur (C) and tibia (D); a higher score indicates that trabeculae are more perpendicular to the bone's long-axis. indicates that trabeculae tend to be more closely associated with other similar trabeculae. Major axis regression lines are also plotted when correlations were statistically significant. $N$ signifies 
3381 number of species represented, and $n$ signifies number of individuals. Other statistical metrics for 3382 each comparison are reported in Table 2.

3383

3384

3385

Figure 43. Gently oblique trabeculae in the tibial diaphysis of a human. Inset shows location of 3386 section relative to the whole bone. Although the trabeculae (or endosteal 'wrinkles') are less obliquely oriented compared to the birds or reptiles, they are nonetheless consistently oriented in an oblique fashion.

Figure 44. Summary of the main cancellous bone architectural patterns observed in the theropods studied, placed in context of their phylogenetic relationships. Architectural patterns were discretized into six multistate characters, and optimized to phylogeny using equal-weighted, parsimony-based ancestral state reconstruction in Mesquite 3.40 (https://www.mesquiteproject.org/). Ancestral states are shown for each node, and character state changes along branches leading to terminal taxa are given in boxes. Characters (as letters) and states (as numbers) were defined as follows: A0, $\mathbf{u}_{1}$ in the femoral head has little anterior inclination; A1, $\mathbf{u}_{1}$ in femoral head has significant anterior inclination; A2, $\mathbf{u}_{1}$ in femoral head is oriented anteroposteriorly; B0, $\mathbf{u}_{1}$ in femoral condyles has small posterior inclination, with minimal anteroposterior sweeping; B1, $\mathbf{u}_{1}$ in femoral condyles has markedly increased posterior inclination; B2, $\mathbf{u}_{1}$ in femoral condyles often has strong posterior inclination, with substantial anteroposterior sweeping; $\mathrm{C}$, coronal-plane double arcuate pattern present in proximal femur; C1, proximal femur lacks coronal-plane double arcuate pattern; D0, sagittal-plane double arcuate pattern present in proximal tibia between condyles and cnemial crest; D1, sagittal-plane doublearcuate pattern in proximal tibia not as widely observed (present only in larger bones); E0, double set of double-arcuate patterns in distal tibia, juxtaposed with highly anisotropic, sagittally aligned fabric in proximal tarsals; E1, highly anisotropic, sagittally aligned fabric throughout both distal tibia and proximal tarsals; F0, fabric near diaphyses is subparallel to bone's long-axis; F1, increased obliquity of fabric nearer the femoral diaphysis; F2, fabric strongly oblique near femoral diaphysis, and oblique trabeculae can extensively invade femoral diaphysis. 
3412

3413 Figure 45. Schematic demonstrating the effect of differences in the degree of hip and knee 3414 flexion on the joint forces experienced by the femur. This is illustrated with right lateral views of 3415 a human (A) and a typical bird (B) in approximate mid-stance postures. In the more flexed 3416 posture of birds, the hip joint force is more anteriorly oriented relative to the long-axis of the 3417 femur (dotted line) compared to humans. Further, the knee joint force is more posteriorly oriented 3418 relative to the long-axis of the femur compared to humans. 


\section{Table $\mathbf{1}$ (on next page)}

The specimens investigated.

In addition to providing the higher-level taxonomy of the species studied (cf. Fig. 8), this table also lists the settings used in acquiring the $\mathrm{CT}$ scan data for each specimen, as well as the protocol used to process the raw CT images for subsequent analysis.

* Collection number abbreviations: AM R., Australian Museum; DNM, UMNH VP; Natural History Museum of Utah; FMNH PR, Field Museum of Natural History; GU, Griffith University teaching collection; MOR, Museum of the Rockies; MVB, MV R., Museum Victoria; PJB, P.J.B. personal collection (housed within Queensland Museum collections); QMJ, QMO, Queensland Museum; RVC, Royal Veterinary College; TMP, Royal Tyrrell Museum of Palaeontology; UA, Université d'Antananarivo; UMZC, Cambridge University Museum of Zoology; YPM, Yale Peabody Museum of Natural History.

** The scans of these specimens were qualitatively analysed only, and did not require any further processing.

${ }^{p}=$ proximal end, ${ }^{d}=$ distal end, ${ }^{s}=$ shaft. 
Table 1. The specimens investigated. In addition to providing the higher-level taxonomy of the species studied (cf. Fig. 8), this table also lists the settings used in acquiring the CT scan data for each specimen, as well as the protocol used to process the raw CT images for subsequent analysis.

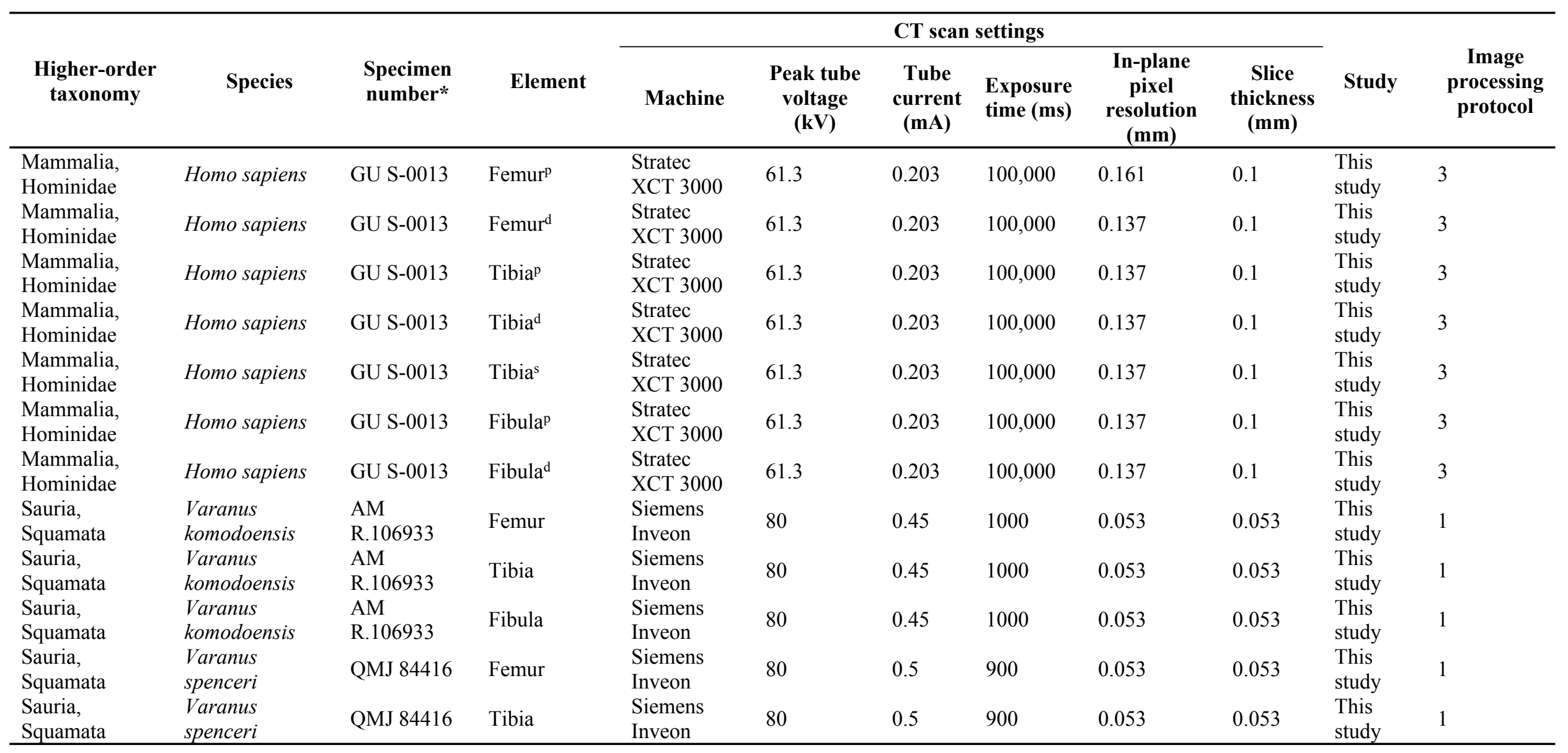




\begin{tabular}{|c|c|c|c|c|c|c|c|c|c|c|c|}
\hline \multirow{2}{*}{\multicolumn{2}{|c|}{$\begin{array}{l}\text { Table } 1 \text { (continued). } \\
\text { Higher-order } \\
\text { taxonomy }\end{array}$}} & \multirow[b]{2}{*}{$\begin{array}{l}\text { Specimen } \\
\text { number* }\end{array}$} & \multirow[b]{2}{*}{ Element } & \multicolumn{6}{|c|}{ CT scan settings } & \multirow[b]{2}{*}{ Study } & \multirow[b]{2}{*}{$\begin{array}{c}\text { Image } \\
\text { processing } \\
\text { protocol }\end{array}$} \\
\hline & & & & Machine & $\begin{array}{l}\text { Peak tube } \\
\text { voltage } \\
(\mathrm{kV})\end{array}$ & $\begin{array}{l}\text { Tube } \\
\text { current } \\
(\mathrm{mA})\end{array}$ & $\begin{array}{l}\text { Exposure } \\
\text { time }(\mathrm{ms})\end{array}$ & $\begin{array}{c}\text { In-plane } \\
\text { pixel } \\
\text { resolution } \\
(\mathbf{m m}) \\
\end{array}$ & $\begin{array}{c}\text { Slice } \\
\text { thickness } \\
(\mathbf{m m})\end{array}$ & & \\
\hline Sauria, Squamata & $\begin{array}{l}\text { Varanus } \\
\text { spenceri }\end{array}$ & QMJ 84416 & Fibula & $\begin{array}{l}\text { Siemens } \\
\text { Inveon }\end{array}$ & 80 & 0.5 & 900 & 0.053 & 0.053 & $\begin{array}{l}\text { This } \\
\text { study }\end{array}$ & 1 \\
\hline Sauria, Squamata & $\begin{array}{l}\text { Varanus } \\
\text { panoptes }\end{array}$ & QMJ 91981 & Femur & $\begin{array}{l}\text { Siemens } \\
\text { Inveon }\end{array}$ & 80 & 0.5 & 900 & 0.053 & 0.053 & $\begin{array}{l}\text { This } \\
\text { study }\end{array}$ & 1 \\
\hline Sauria, Squamata & $\begin{array}{l}\text { Varanus } \\
\text { panoptes }\end{array}$ & QMJ 91981 & Tibia & $\begin{array}{l}\text { Siemens } \\
\text { Inveon }\end{array}$ & 80 & 0.5 & 900 & 0.053 & 0.053 & $\begin{array}{l}\text { This } \\
\text { study }\end{array}$ & 1 \\
\hline Sauria, Squamata & $\begin{array}{l}\text { Varanus } \\
\text { panoptes }\end{array}$ & QMJ 91981 & Fibula & $\begin{array}{l}\text { Siemens } \\
\text { Inveon }\end{array}$ & 80 & 0.5 & 900 & 0.053 & 0.053 & $\begin{array}{l}\text { This } \\
\text { study }\end{array}$ & 1 \\
\hline $\begin{array}{l}\text { Archosauria, } \\
\text { Crocodylia }\end{array}$ & $\begin{array}{l}\text { Crocodylus } \\
\text { johnstoni }\end{array}$ & QMJ 47916 & Femur & $\begin{array}{l}\text { Siemens } \\
\text { Inveon }\end{array}$ & 80 & 0.5 & 900 & 0.053 & 0.053 & $\begin{array}{l}\text { This } \\
\text { study }\end{array}$ & 1 \\
\hline $\begin{array}{l}\text { Archosauria, } \\
\text { Crocodylia }\end{array}$ & $\begin{array}{l}\text { Crocodylus } \\
\text { johnstoni }\end{array}$ & QMJ 47916 & Tibia & $\begin{array}{l}\text { Siemens } \\
\text { Inveon }\end{array}$ & 80 & 0.5 & 900 & 0.053 & 0.053 & $\begin{array}{l}\text { This } \\
\text { study }\end{array}$ & 1 \\
\hline $\begin{array}{l}\text { Archosauria, } \\
\text { Crocodylia }\end{array}$ & $\begin{array}{l}\text { Crocodylus } \\
\text { johnstoni }\end{array}$ & QMJ 47916 & Fibula & $\begin{array}{l}\text { Siemens } \\
\text { Inveon }\end{array}$ & 80 & 0.5 & 900 & 0.053 & 0.053 & $\begin{array}{l}\text { This } \\
\text { study }\end{array}$ & 1 \\
\hline $\begin{array}{l}\text { Archosauria, } \\
\text { Crocodylia }\end{array}$ & $\begin{array}{l}\text { Crocodylus } \\
\text { porosus }\end{array}$ & QMJ 48127 & Femur & $\begin{array}{l}\text { Siemens } \\
\text { Inveon }\end{array}$ & 80 & 0.45 & 1000 & 0.053 & 0.053 & $\begin{array}{l}\text { This } \\
\text { study }\end{array}$ & 1 \\
\hline $\begin{array}{l}\text { Archosauria, } \\
\text { Crocodylia }\end{array}$ & $\begin{array}{l}\text { Crocodylus } \\
\text { porosus }\end{array}$ & QMJ 48127 & Tibia & $\begin{array}{l}\text { Siemens } \\
\text { Inveon }\end{array}$ & 80 & 0.45 & 1000 & 0.053 & 0.053 & $\begin{array}{l}\text { This } \\
\text { study }\end{array}$ & 1 \\
\hline $\begin{array}{l}\text { Archosauria, } \\
\text { Crocodylia }\end{array}$ & $\begin{array}{l}\text { Crocodylus } \\
\text { porosus }\end{array}$ & QMJ 48127 & Fibula & $\begin{array}{l}\text { Siemens } \\
\text { Inveon }\end{array}$ & 80 & 0.45 & 1000 & 0.053 & 0.053 & $\begin{array}{l}\text { This } \\
\text { study }\end{array}$ & 1 \\
\hline $\begin{array}{l}\text { Non-avian } \\
\text { theropod, } \\
\text { Ceratosauria }\end{array}$ & $\begin{array}{l}\text { Ceratosaurus } \\
\text { nasicornis }\end{array}$ & $\begin{array}{l}\text { UMNH VP } \\
5278\end{array}$ & $\begin{array}{l}\text { Tibia }+ \\
\text { astragalus }+ \\
\text { calcaneum }\end{array}$ & $\begin{array}{l}\text { Siemens } \\
\text { Somatom } \\
\text { Definition } \\
\text { Flash }\end{array}$ & 80,140 & 480 & 500 & 0.504 & 0.5 & $\begin{array}{l}\text { This } \\
\text { study }\end{array}$ & 5 \\
\hline $\begin{array}{l}\text { Non-avian } \\
\text { theropod, } \\
\text { Ceratosauria }\end{array}$ & $\begin{array}{l}\text { Masiakasaurus } \\
\text { knopfleri }\end{array}$ & $\begin{array}{l}\text { FMNH PR } \\
2117\end{array}$ & Femur & $\begin{array}{l}\text { GE } \\
\text { Lightspeed } \\
16\end{array}$ & 100 & 70 & 1297 & 0.1875 & 1 & $\begin{array}{l}\text { Farke } \\
\text { and } \\
\text { Alicea } \\
(2009)\end{array}$ & 2 \\
\hline $\begin{array}{l}\text { Non-avian } \\
\text { theropod, } \\
\text { Ceratosauria }\end{array}$ & $\begin{array}{l}\text { Masiakasaurus } \\
\text { knopfleri }\end{array}$ & $\begin{array}{l}\text { FMNH PR } \\
2153\end{array}$ & Femur & $\begin{array}{l}\text { GE } \\
\text { Lightspeed } \\
16\end{array}$ & 100 & 100 & 2101 & 0.1875 & 1.338 & $\begin{array}{l}\text { Farke } \\
\text { and } \\
\text { Alicea } \\
(2009)\end{array}$ & 2 \\
\hline
\end{tabular}


Table 1 (continued).

\begin{tabular}{|c|c|c|c|c|c|c|c|c|c|c|c|}
\hline \multirow[b]{2}{*}{$\begin{array}{l}\text { Higher-order } \\
\text { taxonomy }\end{array}$} & \multirow[b]{2}{*}{ Species } & \multirow[b]{2}{*}{$\begin{array}{l}\text { Specimen } \\
\text { number* }\end{array}$} & \multirow[b]{2}{*}{ Element } & \multicolumn{6}{|c|}{ CT scan settings } & \multirow[b]{2}{*}{ Study } & \multirow[b]{2}{*}{$\begin{array}{c}\text { Image } \\
\text { processing } \\
\text { protocol }\end{array}$} \\
\hline & & & & Machine & $\begin{array}{c}\text { Peak tube } \\
\text { voltage } \\
(\mathbf{k V})\end{array}$ & $\begin{array}{c}\text { Tube } \\
\text { current } \\
(\mathbf{m A})\end{array}$ & $\begin{array}{l}\text { Exposure } \\
\text { time (ms) }\end{array}$ & $\begin{array}{c}\text { In-plane } \\
\text { pixel } \\
\text { resolution } \\
(\mathrm{mm}) \\
\end{array}$ & $\begin{array}{c}\text { Slice } \\
\text { thickness } \\
(\mathrm{mm})\end{array}$ & & \\
\hline $\begin{array}{l}\text { Non-avian } \\
\text { theropod, } \\
\text { Ceratosauria }\end{array}$ & $\begin{array}{l}\text { Masiakasaurus } \\
\text { knopfleri }\end{array}$ & $\begin{array}{l}\text { FMNH PR } \\
2208\end{array}$ & Femur & $\begin{array}{l}\text { GE } \\
\text { Lightspeed } \\
16\end{array}$ & 100 & 70 & 1297 & 0.1875 & 1 & $\begin{array}{l}\text { Farke } \\
\text { and } \\
\text { Alicea } \\
(2009)\end{array}$ & 2 \\
\hline $\begin{array}{l}\text { Non-avian } \\
\text { theropod, } \\
\text { Ceratosauria }\end{array}$ & $\begin{array}{l}\text { Masiakasaurus } \\
\text { knopfleri }\end{array}$ & UA 8684 & Femur & $\begin{array}{l}\text { GE } \\
\text { Lightspeed } \\
16\end{array}$ & 100 & 70 & 1297 & 0.1875 & 1 & $\begin{array}{l}\text { Farke } \\
\text { and } \\
\text { Alicea } \\
\text { (2009) }\end{array}$ & 2 \\
\hline $\begin{array}{l}\text { Non-avian } \\
\text { theropod, } \\
\text { Allosauroidea }\end{array}$ & Allosaurus sp. & MOR 693 & Femora $\times 2$ & $\begin{array}{l}\text { Toshiba } \\
\text { Aquilion } 64\end{array}$ & 135 & 250 & 750 & 0.625 & 0.5 & $\begin{array}{l}\text { This } \\
\text { study }\end{array}$ & 5 \\
\hline $\begin{array}{l}\text { Non-avian } \\
\text { theropod, } \\
\text { Allosauroidea }\end{array}$ & Allosaurus sp. & MOR 693 & Tibiae $\times 2$ & $\begin{array}{l}\text { Toshiba } \\
\text { Aquilion } 64\end{array}$ & 135 & 300 & 750 & 0.468 & 0.4 & $\begin{array}{l}\text { This } \\
\text { study }\end{array}$ & 5 \\
\hline $\begin{array}{l}\text { Non-avian } \\
\text { theropod, } \\
\text { Allosauroidea }\end{array}$ & Allosaurus sp. & MOR 693 & Fibulae $\times 2$ & $\begin{array}{l}\text { Toshiba } \\
\text { Aquilion } 64\end{array}$ & 135 & 250 & 750 & 0.625 & 0.5 & $\begin{array}{l}\text { This } \\
\text { study }\end{array}$ & 5 \\
\hline $\begin{array}{l}\text { Non-avian } \\
\text { theropod, } \\
\text { Allosauroidea }\end{array}$ & Allosaurus sp. & MOR 693 & Astragalus & $\begin{array}{l}\text { Toshiba } \\
\text { Aquilion } 64\end{array}$ & 135 & 250 & 750 & 0.625 & 0.5 & $\begin{array}{l}\text { This } \\
\text { study }\end{array}$ & 5 \\
\hline $\begin{array}{l}\text { Non-avian } \\
\text { theropod, } \\
\text { Allosauroidea }\end{array}$ & Allosaurus sp. & MOR 693 & Calcaneum & $\begin{array}{l}\text { Toshiba } \\
\text { Aquilion } 64\end{array}$ & 135 & 250 & 750 & 0.625 & 0.5 & $\begin{array}{l}\text { This } \\
\text { study }\end{array}$ & 5 \\
\hline $\begin{array}{l}\text { Non-avian } \\
\text { theropod, } \\
\text { Allosauroidea }\end{array}$ & $\begin{array}{l}\text { Allosaurus } \\
\text { fragilis }\end{array}$ & DNM 2560 & Femur & $\begin{array}{l}\text { Siemens } \\
\text { Somatom } \\
\text { Definition } \\
\text { Flash }\end{array}$ & 80,140 & 315 & 500 & 0.549 & 0.5 & $\begin{array}{l}\text { This } \\
\text { study }\end{array}$ & 5 \\
\hline
\end{tabular}




\begin{tabular}{lllllllll}
$\begin{array}{l}\text { Non-avian } \\
\text { theropod, } \\
\text { Allosauroidea }\end{array}$ & $\begin{array}{l}\text { Allosaurus } \\
\text { fragilis }\end{array}$ & DNM 2560 & $\begin{array}{l}\text { Tibia }+ \\
\text { astragalus }+ \\
\text { calcaneum }\end{array}$ & $\begin{array}{l}\text { Siemens } \\
\text { Somatom } \\
\text { Definition } \\
\text { Flash }\end{array}$ & 80,140 & 360 & 500 & 0.637 \\
\hline
\end{tabular}

\begin{tabular}{|c|c|c|c|c|c|c|c|c|c|c|c|}
\hline \multirow[b]{2}{*}{$\begin{array}{l}\text { Higher-order } \\
\text { taxonomy }\end{array}$} & \multirow[b]{2}{*}{ Species } & \multirow[b]{2}{*}{$\begin{array}{l}\text { Specimen } \\
\text { number* }\end{array}$} & \multirow[b]{2}{*}{ Element } & \multicolumn{6}{|c|}{ CT scan settings } & \multirow[b]{2}{*}{ Study } & \multirow[b]{2}{*}{$\begin{array}{c}\text { Image } \\
\text { processing } \\
\text { protocol }\end{array}$} \\
\hline & & & & Machine & $\begin{array}{c}\text { Peak tube } \\
\text { voltage } \\
(\mathrm{kV})\end{array}$ & $\begin{array}{c}\text { Tube } \\
\text { current } \\
(\mathrm{mA})\end{array}$ & $\begin{array}{l}\text { Exposure } \\
\text { time }(\mathrm{ms})\end{array}$ & $\begin{array}{c}\text { In-plane } \\
\text { pixel } \\
\text { resolution } \\
(\mathbf{m m}) \\
\end{array}$ & $\begin{array}{c}\begin{array}{c}\text { Slice } \\
\text { thickness } \\
(\mathbf{m m})\end{array} \\
\end{array}$ & & \\
\hline $\begin{array}{l}\text { Non-avian } \\
\text { theropod, } \\
\text { Allosauroidea }\end{array}$ & $\begin{array}{l}\text { Allosaurus } \\
\text { fragilis }\end{array}$ & $\begin{array}{l}\text { UMNH VP } \\
7884\end{array}$ & Femur & $\begin{array}{l}\text { Siemens } \\
\text { Somatom } \\
\text { Definition } \\
\text { Flash }\end{array}$ & 80,140 & 630 & 500 & 0.529 & 0.5 & $\begin{array}{l}\text { This } \\
\text { study }\end{array}$ & 5 \\
\hline $\begin{array}{l}\text { Non-avian } \\
\text { theropod, } \\
\text { Allosauroidea }\end{array}$ & $\begin{array}{l}\text { Allosaurus } \\
\text { fragilis }\end{array}$ & $\begin{array}{l}\text { UMNH VP } \\
7885\end{array}$ & Femur & $\begin{array}{l}\text { Siemens } \\
\text { Somatom } \\
\text { Definition } \\
\text { Flash }\end{array}$ & 80,140 & 225 & 500 & 0.387 & 0.5 & $\begin{array}{l}\text { This } \\
\text { study }\end{array}$ & 5 \\
\hline $\begin{array}{l}\text { Non-avian } \\
\text { theropod, } \\
\text { Allosauroidea }\end{array}$ & $\begin{array}{l}\text { Allosaurus } \\
\text { fragilis }\end{array}$ & $\begin{array}{l}\text { UMNH VP } \\
7889\end{array}$ & Femur $^{\mathrm{p}}$ & $\begin{array}{l}\text { Siemens } \\
\text { Somatom } \\
\text { Definition } \\
\text { Flash }\end{array}$ & 80,140 & 630 & 500 & 0.523 & 0.5 & $\begin{array}{l}\text { This } \\
\text { study }\end{array}$ & 5 \\
\hline $\begin{array}{l}\text { Non-avian } \\
\text { theropod, } \\
\text { Allosauroidea }\end{array}$ & $\begin{array}{l}\text { Allosaurus } \\
\text { fragilis }\end{array}$ & $\begin{array}{l}\text { UMNH VP } \\
7928\end{array}$ & Tibia & $\begin{array}{l}\text { Siemens } \\
\text { Somatom } \\
\text { Definition } \\
\text { Flash }\end{array}$ & 80,140 & 630 & 500 & 0.355 & 0.5 & $\begin{array}{l}\text { This } \\
\text { study }\end{array}$ & 5 \\
\hline $\begin{array}{l}\text { Non-avian } \\
\text { theropod, } \\
\text { Allosauroidea }\end{array}$ & $\begin{array}{l}\text { Allosaurus } \\
\text { fragilis }\end{array}$ & $\begin{array}{l}\text { UMNH VP } \\
9480\end{array}$ & Femur & $\begin{array}{l}\text { Siemens } \\
\text { Somatom } \\
\text { Definition } \\
\text { Flash }\end{array}$ & 80,140 & 80 & 500 & 0.217 & 0.5 & $\begin{array}{l}\text { This } \\
\text { study }\end{array}$ & 5 \\
\hline $\begin{array}{l}\text { Non-avian } \\
\text { theropod, }\end{array}$ & $\begin{array}{l}\text { Allosaurus } \\
\text { fragilis }\end{array}$ & $\begin{array}{l}\text { UMNH VP } \\
20363\end{array}$ & Femur & $\begin{array}{l}\text { Siemens } \\
\text { Somatom }\end{array}$ & 80,140 & 315 & 500 & 0.664 & 0.5 & $\begin{array}{l}\text { This } \\
\text { study }\end{array}$ & 5 \\
\hline
\end{tabular}


Allosauroidea

Table 1 (continued).

\section{Non-avian}

theropod,

Allosauroidea

Allosaurus

fragilis

24326

Non-avian

theropod,

Tyrannosaurus

rex

Tyrannosauridae
Definition

Flash

Siemens

Somatom

Definition

80,140

Flash

Toshiba

Aquilion 64
135

\begin{tabular}{rrrrrll}
140 & 200 & 500 & 0.459 & 0.5 & $\begin{array}{l}\text { This } \\
\text { study }\end{array}$ & 5 \\
& 500 & 500 & 0.782 & 0.4 & $\begin{array}{l}\text { This } \\
\text { study }\end{array}$ & 5 \\
\hline
\end{tabular}

Table 1 (continued). 


\begin{tabular}{|c|c|c|c|c|c|c|c|c|c|c|c|}
\hline \multirow[b]{2}{*}{$\begin{array}{l}\text { Higher-order } \\
\text { taxonomy }\end{array}$} & \multirow[b]{2}{*}{ Species } & \multirow[b]{2}{*}{$\begin{array}{l}\text { Specimen } \\
\text { number* }\end{array}$} & \multirow[b]{2}{*}{ Element } & \multicolumn{6}{|c|}{ CT scan settings } & \multirow[b]{2}{*}{ Study } & \multirow[b]{2}{*}{$\begin{array}{c}\text { Image } \\
\text { processing } \\
\text { protocol }\end{array}$} \\
\hline & & & & Machine & $\begin{array}{l}\text { Peak tube } \\
\text { voltage } \\
(\mathbf{k V})\end{array}$ & $\begin{array}{l}\text { Tube } \\
\text { current } \\
(\mathbf{m A})\end{array}$ & $\begin{array}{l}\text { Exposure } \\
\text { time (ms) }\end{array}$ & $\begin{array}{c}\text { In-plane } \\
\text { pixel } \\
\text { resolution } \\
(\mathbf{m m}) \\
\end{array}$ & $\begin{array}{c}\text { Slice } \\
\text { thickness } \\
(\mathbf{m m})\end{array}$ & & \\
\hline $\begin{array}{l}\text { Non-avian } \\
\text { theropod, } \\
\text { Tyrannosauridae }\end{array}$ & $\begin{array}{l}\text { Tyrannosaurus } \\
\text { rex }\end{array}$ & MOR 1125 & Femur & $\begin{array}{l}\text { Toshiba } \\
\text { Aquilion } 64\end{array}$ & 135 & 350 & 500 & 1.178 & 0.5 & $\begin{array}{l}\text { This } \\
\text { study }\end{array}$ & 5 \\
\hline $\begin{array}{l}\text { Non-avian } \\
\text { theropod, } \\
\text { Tyrannosauridae }\end{array}$ & $\begin{array}{l}\text { Tyrannosaurus } \\
\text { rex }\end{array}$ & MOR 1125 & Tibia & $\begin{array}{l}\text { Toshiba } \\
\text { Aquilion } 64\end{array}$ & 135 & 350 & 500 & 0.976 & 0.5 & $\begin{array}{l}\text { This } \\
\text { study }\end{array}$ & 5 \\
\hline $\begin{array}{l}\text { Non-avian } \\
\text { theropod, } \\
\text { Tyrannosauridae }\end{array}$ & $\begin{array}{l}\text { Tyrannosaurus } \\
\text { rex }\end{array}$ & MOR 1125 & Fibulae $\times 2$ & $\begin{array}{l}\text { Toshiba } \\
\text { Aquilion } 64\end{array}$ & 135 & 350 & 500 & 0.873 & 0.8 & $\begin{array}{l}\text { This } \\
\text { study }\end{array}$ & 5 \\
\hline $\begin{array}{l}\text { Non-avian } \\
\text { theropod, } \\
\text { Tyrannosauridae }\end{array}$ & $\begin{array}{l}\text { Tyrannosaurus } \\
\text { rex }\end{array}$ & MOR 1128 & Femur & $\begin{array}{l}\text { Toshiba } \\
\text { Aquilion } 64\end{array}$ & 135 & 350 & 500 & 0.976 & 2 & $\begin{array}{l}\text { This } \\
\text { study }\end{array}$ & 5 \\
\hline $\begin{array}{l}\text { Non-avian } \\
\text { theropod, } \\
\text { Tyrannosauridae }\end{array}$ & $\begin{array}{l}\text { Tyrannosaurus } \\
\text { rex }\end{array}$ & MOR 1128 & Tibia $^{\mathrm{d}}$ & $\begin{array}{l}\text { Toshiba } \\
\text { Aquilion } 64\end{array}$ & 135 & 350 & 500 & 0.961 & 0.5 & $\begin{array}{l}\text { This } \\
\text { study }\end{array}$ & 5 \\
\hline $\begin{array}{l}\text { Non-avian } \\
\text { theropod, } \\
\text { Tyrannosauridae }\end{array}$ & $\begin{array}{l}\text { Tyrannosauridae } \\
\text { indet. }\end{array}$ & MOR 1192 & Fibula & $\begin{array}{l}\text { Toshiba } \\
\text { Aquilion } 64\end{array}$ & 135 & 150 & 1000 & 0.976 & 2 & $\begin{array}{l}\text { This } \\
\text { study }\end{array}$ & 5 \\
\hline $\begin{array}{l}\text { Non-avian } \\
\text { theropod, } \\
\text { Tyrannosauridae }\end{array}$ & $\begin{array}{l}\text { Gorgosaurus } \\
\text { libratus }\end{array}$ & $\begin{array}{l}\text { TMP } \\
1994.012 .0602\end{array}$ & Femur & $\begin{array}{l}\text { GE } \\
\text { Lightspeed } \\
\text { Ultra }\end{array}$ & 120 & 160 & 1195 & 0.723 & 1.25 & $\begin{array}{l}\text { This } \\
\text { study }\end{array}$ & 5 \\
\hline $\begin{array}{l}\text { Non-avian } \\
\text { theropod, } \\
\text { Tyrannosauridae }\end{array}$ & $\begin{array}{l}\text { Gorgosaurus } \\
\text { libratus }\end{array}$ & $\begin{array}{l}\text { TMP } \\
1994.012 .0602\end{array}$ & $\begin{array}{l}\text { Tibia }+ \\
\text { astragalus }+ \\
\text { calcaneum }\end{array}$ & $\begin{array}{l}\text { GE } \\
\text { Lightspeed } \\
\text { Ultra }\end{array}$ & 140 & 150 & 1195 & 0.703 & 1.25 & $\begin{array}{l}\text { This } \\
\text { study }\end{array}$ & 5 \\
\hline $\begin{array}{l}\text { Non-avian } \\
\text { theropod, } \\
\text { Tyrannosauridae }\end{array}$ & $\begin{array}{l}\text { Daspletosaurus } \\
\text { torosus }\end{array}$ & $\begin{array}{l}\text { TMP } \\
2001.036 .0001\end{array}$ & Femur & $\begin{array}{l}\text { GE } \\
\text { Lightspeed } \\
\text { Ultra }\end{array}$ & 140 & 150 & 1195 & 0.838 & 1.25 & $\begin{array}{l}\text { This } \\
\text { study }\end{array}$ & 5 \\
\hline $\begin{array}{l}\text { Non-avian } \\
\text { theropod, } \\
\text { Tyrannosauridae }\end{array}$ & $\begin{array}{l}\text { Daspletosaurus } \\
\text { torosus }\end{array}$ & $\begin{array}{l}\text { TMP } \\
2001.036 .0001\end{array}$ & Tibia & $\begin{array}{l}\text { GE } \\
\text { Lightspeed } \\
\text { Ultra }\end{array}$ & 120 & 245 & 1195 & 0.832 & 1.25 & $\begin{array}{l}\text { This } \\
\text { study }\end{array}$ & 5 \\
\hline
\end{tabular}

Table 1 (continued). 


\begin{tabular}{|c|c|c|c|c|c|c|c|c|c|c|c|}
\hline \multirow[b]{2}{*}{$\begin{array}{l}\text { Higher-order } \\
\text { taxonomy }\end{array}$} & \multirow[b]{2}{*}{ Species } & \multirow[b]{2}{*}{$\begin{array}{l}\text { Specimen } \\
\text { number* }\end{array}$} & \multirow[b]{2}{*}{ Element } & \multicolumn{6}{|c|}{ CT scan settings } & \multirow[b]{2}{*}{ Study } & \multirow[b]{2}{*}{$\begin{array}{c}\text { Image } \\
\text { processing } \\
\text { protocol }\end{array}$} \\
\hline & & & & Machine & $\begin{array}{c}\text { Peak tube } \\
\text { voltage } \\
(\mathbf{k V})\end{array}$ & $\begin{array}{c}\begin{array}{c}\text { Tube } \\
\text { current } \\
(\mathrm{mA})\end{array} \\
\end{array}$ & $\begin{array}{l}\text { Exposure } \\
\text { time }(\mathrm{ms})\end{array}$ & $\begin{array}{c}\text { In-plane } \\
\text { pixel } \\
\text { resolution } \\
(\mathbf{m m}) \\
\end{array}$ & $\begin{array}{c}\text { Slice } \\
\text { thickness } \\
(\mathrm{mm})\end{array}$ & & \\
\hline $\begin{array}{l}\text { Non-avian } \\
\text { theropod, } \\
\text { Tyrannosauridae }\end{array}$ & $\begin{array}{l}\text { Daspletosaurus } \\
\text { torosus }\end{array}$ & $\begin{array}{l}\text { TMP } \\
2001.036 .0001\end{array}$ & Fibula & $\begin{array}{l}\text { GE } \\
\text { Lightspeed } \\
\text { Ultra }\end{array}$ & 120 & 245 & 1195 & 0.832 & 1.25 & $\begin{array}{l}\text { This } \\
\text { study }\end{array}$ & 5 \\
\hline $\begin{array}{l}\text { Non-avian } \\
\text { theropod, } \\
\text { Tyrannosauridae }\end{array}$ & $\begin{array}{l}\text { Daspletosaurus } \\
\text { torosus }\end{array}$ & $\begin{array}{l}\text { TMP } \\
2001.036 .0001\end{array}$ & Astragalus & $\begin{array}{l}\text { GE } \\
\text { Lightspeed } \\
\text { Ultra }\end{array}$ & 140 & 155 & 1195 & 0.879 & 1.25 & $\begin{array}{l}\text { This } \\
\text { study }\end{array}$ & 5 \\
\hline $\begin{array}{l}\text { Non-avian } \\
\text { theropod, } \\
\text { Ornithomimidae }\end{array}$ & $\begin{array}{l}\text { Ornithomimidae } \\
\text { indet. }\end{array}$ & $\begin{array}{l}\text { TMP } \\
1985.036 .0276\end{array}$ & Femur $^{p}$ & $\begin{array}{l}\text { Siemens } \\
\text { Inveon }\end{array}$ & 80 & 500 & 825 & 0.05 & 0.05 & $\begin{array}{l}\text { This } \\
\text { study }\end{array}$ & 4 \\
\hline $\begin{array}{l}\text { Non-avian } \\
\text { theropod, } \\
\text { Ornithomimidae }\end{array}$ & $\begin{array}{l}\text { Ornithomimidae } \\
\text { indet. }\end{array}$ & $\begin{array}{l}\text { TMP } \\
1991.036 .0569\end{array}$ & Femur & $\begin{array}{l}\text { Siemens } \\
\text { Inveon }\end{array}$ & 80 & 250 & 1500 & 0.05 & 0.05 & $\begin{array}{l}\text { This } \\
\text { study }\end{array}$ & 4 \\
\hline $\begin{array}{l}\text { Non-avian } \\
\text { theropod, } \\
\text { Ornithomimidae }\end{array}$ & $\begin{array}{l}\text { Ornithomimidae } \\
\text { indet. }\end{array}$ & $\begin{array}{l}\text { TMP } \\
1991.036 .0854\end{array}$ & Femurp $^{p}$ & $\begin{array}{l}\text { GE } \\
\text { Lightspeed } \\
\text { Ultra }\end{array}$ & 140 & 150 & 1195 & 0.943 & 1.25 & $\begin{array}{l}\text { This } \\
\text { study }\end{array}$ & 5 \\
\hline $\begin{array}{l}\text { Non-avian } \\
\text { theropod, } \\
\text { Ornithomimidae }\end{array}$ & $\begin{array}{l}\text { Ornithomimidae } \\
\text { indet. }\end{array}$ & $\begin{array}{l}\text { TMP } \\
1992.036 .0696\end{array}$ & Femur $^{p}$ & $\begin{array}{l}\text { GE } \\
\text { Lightspeed } \\
\text { Ultra }\end{array}$ & 140 & 150 & 1195 & 0.943 & 1.25 & $\begin{array}{l}\text { This } \\
\text { study }\end{array}$ & 5 \\
\hline $\begin{array}{l}\text { Non-avian } \\
\text { theropod, } \\
\text { Ornithomimidae }\end{array}$ & $\begin{array}{l}\text { Ornithomimidae } \\
\text { indet. }\end{array}$ & $\begin{array}{l}\text { TMP } \\
1993.066 .0002\end{array}$ & Tibia $^{p}$ & $\begin{array}{l}\text { GE } \\
\text { Lightspeed } \\
\text { Ultra }\end{array}$ & 140 & 155 & 1195 & 0.879 & 1.25 & $\begin{array}{l}\text { This } \\
\text { study }\end{array}$ & 5 \\
\hline $\begin{array}{l}\text { Non-avian } \\
\text { theropod, } \\
\text { Ornithomimidae }\end{array}$ & $\begin{array}{l}\text { Ornithomimidae } \\
\text { indet. }\end{array}$ & $\begin{array}{l}\text { TMP } \\
1999.055 .0337\end{array}$ & Femur $^{d}$ & $\begin{array}{l}\text { Siemens } \\
\text { Inveon }\end{array}$ & 80 & 250 & 1500 & 0.05 & 0.05 & $\begin{array}{l}\text { This } \\
\text { study }\end{array}$ & 4 \\
\hline $\begin{array}{l}\text { Non-avian } \\
\text { theropod, } \\
\text { Ornithomimidae }\end{array}$ & $\begin{array}{l}\text { Ornithomimidae } \\
\text { indet. }\end{array}$ & $\begin{array}{l}\text { TMP } \\
2006.012 .0065\end{array}$ & Fibula & $\begin{array}{l}\text { GE } \\
\text { Lightspeed } \\
\text { Ultra }\end{array}$ & 120 & 185 & 1195 & 0.738 & 1.25 & $\begin{array}{l}\text { This } \\
\text { study }\end{array}$ & 5 \\
\hline $\begin{array}{l}\text { Non-avian } \\
\text { theropod, } \\
\text { Therizinosauria }\end{array}$ & $\begin{array}{l}\text { Falcarius } \\
\text { utahensis }\end{array}$ & $\begin{array}{l}\text { UMNH VP } \\
12360\end{array}$ & Femur $^{\mathrm{d}}$ & $\begin{array}{l}\text { Siemens } \\
\text { Inveon }\end{array}$ & 80 & 250 & 1600 & 0.05 & 0.05 & $\begin{array}{l}\text { This } \\
\text { study }\end{array}$ & 4 \\
\hline
\end{tabular}


Table 1 (continued).

\begin{tabular}{|c|c|c|c|c|c|c|c|c|c|c|c|}
\hline \multirow[b]{2}{*}{$\begin{array}{l}\text { Higher-order } \\
\text { taxonomy }\end{array}$} & \multirow[b]{2}{*}{ Species } & \multirow[b]{2}{*}{$\begin{array}{l}\text { Specimen } \\
\text { number* }\end{array}$} & \multirow[b]{2}{*}{ Element } & \multicolumn{6}{|c|}{ CT scan settings } & \multirow[b]{2}{*}{ Study } & \multirow[b]{2}{*}{$\begin{array}{c}\text { Image } \\
\text { processing } \\
\text { protocol }\end{array}$} \\
\hline & & & & Machine & $\begin{array}{c}\text { Peak } \\
\text { tube } \\
\text { voltage } \\
(\mathbf{k V}) \\
\end{array}$ & $\begin{array}{c}\text { Tube } \\
\text { current } \\
(\mathbf{m A})\end{array}$ & $\begin{array}{l}\text { Exposur } \\
\text { e time } \\
\text { (ms) }\end{array}$ & $\begin{array}{c}\text { In-plane } \\
\text { pixel } \\
\text { resolutio } \\
\text { n (mm) } \\
\end{array}$ & $\begin{array}{c}\text { Slice } \\
\text { thicknes } \\
\text { s (mm) }\end{array}$ & & \\
\hline $\begin{array}{l}\text { Non-avian } \\
\text { theropod, } \\
\text { Therizinosauria }\end{array}$ & $\begin{array}{l}\text { Falcarius } \\
\text { utahensis }\end{array}$ & $\begin{array}{l}\text { UMNH VP } \\
12361\end{array}$ & Femur $^{p}$ & $\begin{array}{l}\text { Siemens } \\
\text { Inveon }\end{array}$ & 80 & 250 & 1700 & 0.05 & 0.05 & $\begin{array}{l}\text { This } \\
\text { study }\end{array}$ & 4 \\
\hline $\begin{array}{l}\text { Non-avian } \\
\text { theropod, } \\
\text { Caenagnathidae }\end{array}$ & $\begin{array}{l}\text { Caenagnathidae } \\
\text { indet. }\end{array}$ & $\begin{array}{l}\text { TMP } \\
1986.036 .03 \\
23\end{array}$ & Femur & $\begin{array}{l}\text { Siemens } \\
\text { Inveon }\end{array}$ & 80 & 250 & 1600 & 0.05 & 0.05 & $\begin{array}{l}\text { This } \\
\text { study }\end{array}$ & 4 \\
\hline $\begin{array}{l}\text { Non-avian } \\
\text { theropod, } \\
\text { Dromaeosaurida } \\
\text { e }\end{array}$ & $\begin{array}{l}\text { Saurornitholest } \\
\text { es langstoni }\end{array}$ & MOR 660 & Tibiae $\times 2$ & $\begin{array}{l}\text { Siemens } \\
\text { Inveon }\end{array}$ & 80 & 250 & 1600 & 0.05 & 0.05 & $\begin{array}{l}\text { This } \\
\text { study }\end{array}$ & 4 \\
\hline $\begin{array}{l}\text { Non-avian } \\
\text { theropod, } \\
\text { Troodontidae }\end{array}$ & $\begin{array}{l}\text { Troodontidae } \\
\text { sp. }\end{array}$ & $\begin{array}{l}\text { MOR 553s- } \\
7.11 .91 .41\end{array}$ & Tibia & $\begin{array}{l}\text { Siemens } \\
\text { Inveon }\end{array}$ & 80 & 200 & 1900 & 0.04 & 0.04 & $\begin{array}{l}\text { This } \\
\text { study }\end{array}$ & 4 \\
\hline $\begin{array}{l}\text { Non-avian } \\
\text { theropod, } \\
\text { Troodontidae }\end{array}$ & $\begin{array}{l}\text { Troodontidae } \\
\text { sp. }\end{array}$ & $\begin{array}{l}\text { MOR 553s- } \\
7.28 .91 .239\end{array}$ & Femur & $\begin{array}{l}\text { Siemens } \\
\text { Inveon }\end{array}$ & 80 & 200 & 1800 & 0.04 & 0.04 & $\begin{array}{l}\text { This } \\
\text { study }\end{array}$ & 4 \\
\hline $\begin{array}{l}\text { Non-avian } \\
\text { theropod, } \\
\text { Troodontidae }\end{array}$ & $\begin{array}{l}\text { Troodontidae } \\
\text { sp. }\end{array}$ & $\begin{array}{l}\text { MOR 553s- } \\
8.17 .92 .265\end{array}$ & Fibula & $\begin{array}{l}\text { Siemens } \\
\text { Inveon }\end{array}$ & 80 & 250 & 1600 & 0.04 & 0.04 & $\begin{array}{l}\text { This } \\
\text { study }\end{array}$ & 4 \\
\hline $\begin{array}{l}\text { Non-avian } \\
\text { theropod, } \\
\text { Troodontidae }\end{array}$ & $\begin{array}{l}\text { Troodontidae } \\
\text { sp. }\end{array}$ & MOR 748 & Femur & $\begin{array}{l}\text { Siemens } \\
\text { Inveon }\end{array}$ & 80 & 200 & 1900 & 0.04 & 0.04 & $\begin{array}{l}\text { This } \\
\text { study }\end{array}$ & 4 \\
\hline $\begin{array}{l}\text { Non-avian } \\
\text { theropod, } \\
\text { Troodontidae }\end{array}$ & $\begin{array}{l}\text { Troodontidae } \\
\text { sp. }\end{array}$ & MOR 748 & $\begin{array}{l}\text { Tibia }+ \\
\text { astragalus } \\
+ \\
\text { calcaneum }\end{array}$ & $\begin{array}{l}\text { Siemens } \\
\text { Inveon }\end{array}$ & 80 & 200 & 1900 & 0.04 & 0.04 & $\begin{array}{l}\text { This } \\
\text { study }\end{array}$ & 4 \\
\hline $\begin{array}{l}\text { Aves, } \\
\text { Struthioniforme } \\
\text { S }\end{array}$ & $\begin{array}{l}\text { Struthio } \\
\text { camelus }\end{array}$ & MV R.2385 & Femur & $\begin{array}{l}\text { GE } \\
\text { BrightSpee } \\
\text { d }\end{array}$ & 120 & 55 & 1681 & 0.488 & 0.3 & $\begin{array}{l}\text { This } \\
\text { study }\end{array}$ & 2 \\
\hline
\end{tabular}




\begin{tabular}{|c|c|c|c|c|c|c|c|c|c|c|c|c|}
\hline & $\begin{array}{l}\text { Aves, } \\
\text { Struthioniforme } \\
\text { s }\end{array}$ & $\begin{array}{l}\text { Struthio } \\
\text { camelus }\end{array}$ & MV R.2385 & Tibiotarsus & $\begin{array}{l}\text { Siemens } \\
\text { Somatom } \\
\text { Definition } \\
\text { AS+ }\end{array}$ & 199 & 1000 & 0.363 & 0.4 & $\begin{array}{l}\text { This } \\
\text { study }\end{array}$ & 2 & \\
\hline \multicolumn{13}{|c|}{28} \\
\hline \multicolumn{13}{|c|}{29} \\
\hline \multicolumn{13}{|c|}{30} \\
\hline \multicolumn{13}{|c|}{31} \\
\hline \multicolumn{13}{|c|}{32} \\
\hline & & & & & \multicolumn{6}{|c|}{ CT scan settings } & \multirow[b]{2}{*}{ Study } & \multirow[b]{2}{*}{$\begin{array}{c}\text { Image } \\
\text { processing } \\
\text { protocol }\end{array}$} \\
\hline & $\begin{array}{l}\text { Higher-order } \\
\text { taxonomy }\end{array}$ & Species & $\begin{array}{l}\text { Specimen } \\
\text { number* }\end{array}$ & Element & Machine & $\begin{array}{l}\text { Peak tube } \\
\text { voltage } \\
(\mathrm{kV})\end{array}$ & $\begin{array}{l}\text { Tube } \\
\text { current } \\
(\mathrm{mA})\end{array}$ & $\begin{array}{l}\text { Exposure } \\
\text { time (ms) }\end{array}$ & $\begin{array}{c}\text { In-plane } \\
\text { pixel } \\
\text { resolution } \\
(\mathbf{m m}) \\
\end{array}$ & $\begin{array}{c}\text { Slice } \\
\text { thickness } \\
(\mathbf{m m})\end{array}$ & & \\
\hline & $\begin{array}{l}\text { Aves, } \\
\text { Struthioniformes }\end{array}$ & Struthio camelus & MV R.2385 & Fibula & $\begin{array}{l}\text { GE } \\
\text { BrightSpeed }\end{array}$ & 120 & 55 & 1681 & 0.488 & 0.3 & $\begin{array}{l}\text { This } \\
\text { study }\end{array}$ & 2 \\
\hline & $\begin{array}{l}\text { Aves, } \\
\text { Struthioniformes }\end{array}$ & Struthio camelus & MV R.2711 & Femur & $\begin{array}{l}\text { GE } \\
\text { BrightSpeed }\end{array}$ & 120 & 55 & 1681 & 0.488 & 0.3 & $\begin{array}{l}\text { This } \\
\text { study }\end{array}$ & 2 \\
\hline & $\begin{array}{l}\text { Aves, } \\
\text { Struthioniformes }\end{array}$ & Struthio camelus & MV R.2711 & Tibiotarsus & $\begin{array}{l}\text { GE } \\
\text { BrightSpeed }\end{array}$ & 120 & 55 & 1681 & 0.488 & 0.3 & $\begin{array}{l}\text { This } \\
\text { study }\end{array}$ & 2 \\
\hline & $\begin{array}{l}\text { Aves, } \\
\text { Struthioniformes }\end{array}$ & Struthio camelus & MV R.2711 & Fibula & $\begin{array}{l}\text { GE } \\
\text { BrightSpeed }\end{array}$ & 120 & 55 & 1681 & 0.488 & 0.3 & $\begin{array}{l}\text { This } \\
\text { study }\end{array}$ & 2 \\
\hline & $\begin{array}{l}\text { Aves, } \\
\text { Struthioniformes }\end{array}$ & Struthio camelus & YPM 2124 & Femur & $\begin{array}{l}\text { GE } \\
\text { Lightspeed } \\
16\end{array}$ & 100 & 70 & 1297 & 0.311 & 1.25 & $\begin{array}{l}\text { Farke } \\
\text { and } \\
\text { Alicea } \\
(2009)\end{array}$ & none** \\
\hline & $\begin{array}{l}\text { Aves, } \\
\text { Struthioniformes }\end{array}$ & Struthio camelus & $\begin{array}{l}\text { RVC Ostrich } \\
2\end{array}$ & Femur & $\begin{array}{l}\text { Picker } \\
\text { PQ5000 }\end{array}$ & 120 & 200 & 1000 & 0.391 & 2 & $\begin{array}{l}\text { Doube } \\
\text { et al. } \\
\text { (2012) }\end{array}$ & 2 \\
\hline & $\begin{array}{l}\text { Aves, } \\
\text { Struthioniformes }\end{array}$ & Struthio camelus & $\begin{array}{l}\text { RVC Ostrich } \\
2\end{array}$ & Tibiotarsus & $\begin{array}{l}\text { Picker } \\
\text { PQ5000 }\end{array}$ & 120 & 200 & 1000 & 0.313 & 4 & $\begin{array}{l}\text { Doube } \\
\text { et al. } \\
\text { (2012) }\end{array}$ & 2 \\
\hline & $\begin{array}{l}\text { Aves, } \\
\text { Struthioniformes }\end{array}$ & Struthio camelus & $\begin{array}{l}\text { RVC Ostrich } \\
2\end{array}$ & Fibula & $\begin{array}{l}\text { Picker } \\
\text { PQ5000 }\end{array}$ & 120 & 200 & 1000 & 0.313 & 4 & $\begin{array}{l}\text { Doube } \\
\text { et al. } \\
(2012)\end{array}$ & 2 \\
\hline
\end{tabular}




\begin{tabular}{|c|c|c|c|c|c|c|c|c|c|c|}
\hline $\begin{array}{l}\text { Aves, } \\
\text { Struthioniformes }\end{array}$ & Struthio camelus & $\begin{array}{l}\text { RVC-JRH- } \\
\text { OST } 1\end{array}$ & Femur & $\begin{array}{l}\text { GE } \\
\text { LightSpeed } \\
\text { Pro } 16\end{array}$ & 120 & 200 & 800 & 0.273 & 0.625 & $\begin{array}{l}\text { This } \\
\text { study }\end{array}$ \\
\hline $\begin{array}{l}\text { Aves, } \\
\text { Struthioniformes }\end{array}$ & Struthio camelus & $\begin{array}{l}\text { RVC-JRH- } \\
\text { OST } 1\end{array}$ & Tibiotarsus & $\begin{array}{l}\text { GE } \\
\text { LightSpeed } \\
\text { Pro } 16\end{array}$ & 120 & 200 & 800 & 0.369 & 1.25 & $\begin{array}{l}\text { This } \\
\text { study }\end{array}$ \\
\hline $\begin{array}{l}\text { Aves, } \\
\text { Struthioniformes }\end{array}$ & Struthio camelus & $\begin{array}{l}\text { RVC-JRH- } \\
\text { OST } 1\end{array}$ & Fibula & $\begin{array}{l}\text { GE } \\
\text { LightSpeed } \\
\text { Pro } 16\end{array}$ & 120 & 200 & 800 & 0.369 & 1.25 & $\begin{array}{l}\text { This } \\
\text { study }\end{array}$ \\
\hline
\end{tabular}

\section{Table 1 (continued).}

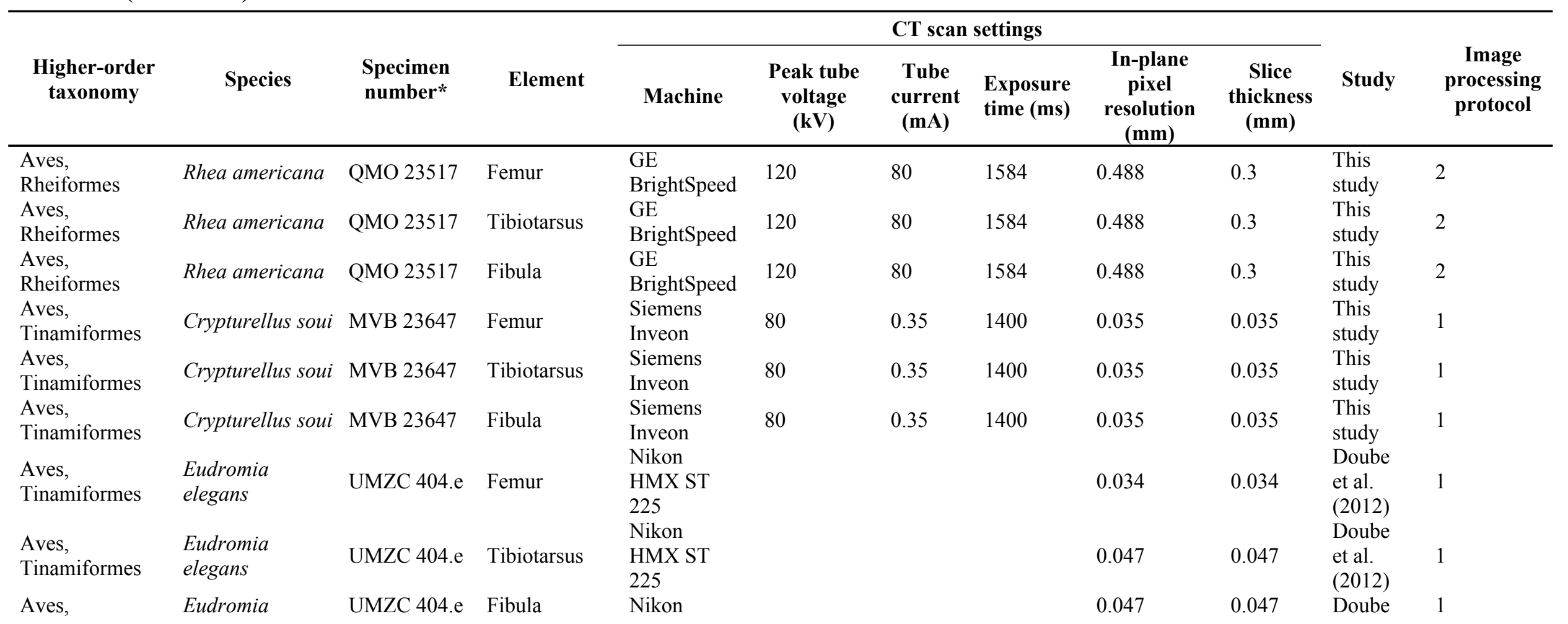




\begin{tabular}{|c|c|c|c|c|c|c|c|c|}
\hline Tinamiformes & elegans & & & $\begin{array}{l}\text { HMX ST } \\
225\end{array}$ & & & $\begin{array}{l}\text { et al. } \\
\text { (2012) }\end{array}$ & \\
\hline $\begin{array}{l}\text { Aves, } \\
\text { Apterygiformes }\end{array}$ & Apteryx owenii & $\begin{array}{l}\text { UMZC } \\
\text { 378.iii }\end{array}$ & Femur & $\begin{array}{l}\text { Nikon } \\
\text { HMX ST } \\
225\end{array}$ & 0.046 & 0.046 & $\begin{array}{l}\text { Doube } \\
\text { et al. } \\
(2012)\end{array}$ & 1 \\
\hline $\begin{array}{l}\text { Aves, } \\
\text { Apterygiformes }\end{array}$ & Apteryx owenii & $\begin{array}{l}\text { UMZC } \\
\text { 378.iii }\end{array}$ & Tibiotarsus & $\begin{array}{l}\text { Nikon } \\
\text { HMX ST } \\
225\end{array}$ & 0.061 & 0.061 & $\begin{array}{l}\text { Doube } \\
\text { et al. } \\
(2012)\end{array}$ & 1 \\
\hline $\begin{array}{l}\text { Aves, } \\
\text { Apterygiformes }\end{array}$ & Apteryx owenii & $\begin{array}{l}\text { UMZC } \\
\text { 378.iii }\end{array}$ & Fibula & $\begin{array}{l}\text { Nikon } \\
\text { HMX ST } \\
225\end{array}$ & 0.061 & 0.061 & $\begin{array}{l}\text { Doube } \\
\text { et al. } \\
(2012)\end{array}$ & 1 \\
\hline
\end{tabular}

\section{Table 1 (continued).}

\begin{tabular}{|c|c|c|c|c|c|c|c|c|c|c|c|}
\hline \multirow[b]{2}{*}{$\begin{array}{l}\text { Higher-order } \\
\text { taxonomy }\end{array}$} & \multirow[b]{2}{*}{ Species } & \multirow[b]{2}{*}{$\begin{array}{l}\text { Specimen } \\
\text { number* }\end{array}$} & \multirow[b]{2}{*}{ Element } & \multicolumn{6}{|c|}{ CT scan settings } & \multirow[b]{2}{*}{ Study } & \multirow[b]{2}{*}{$\begin{array}{c}\text { Image } \\
\text { processing } \\
\text { protocol }\end{array}$} \\
\hline & & & & Machine & $\begin{array}{c}\text { Peak tube } \\
\text { voltage } \\
(\mathrm{kV})\end{array}$ & $\begin{array}{c}\text { Tube } \\
\text { current } \\
(\mathrm{mA})\end{array}$ & $\begin{array}{l}\text { Exposure } \\
\text { time (ms) }\end{array}$ & $\begin{array}{c}\text { In-plane } \\
\text { pixel } \\
\text { resolution } \\
(\mathbf{m m}) \\
\end{array}$ & 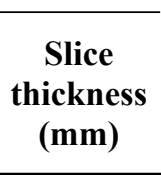 & & \\
\hline $\begin{array}{l}\text { Aves, } \\
\text { Apterygiformes }\end{array}$ & Apteryx haastii & $\begin{array}{l}\text { UMZC } \\
378 . p\end{array}$ & Femur & $\begin{array}{l}\text { Nikon } \\
\text { HMX ST } \\
225\end{array}$ & & & & 0.044 & 0.044 & $\begin{array}{l}\text { Doube } \\
\text { et al. } \\
\text { (2012) }\end{array}$ & 1 \\
\hline $\begin{array}{l}\text { Aves, } \\
\text { Casuariiformes }\end{array}$ & $\begin{array}{l}\text { Dromaius } \\
\text { novaehollandiae }\end{array}$ & QMO 11685 & Femur & $\begin{array}{l}\text { GE } \\
\text { BrightSpeed }\end{array}$ & 120 & 55 & 1681 & 0.379 & 0.3 & $\begin{array}{l}\text { This } \\
\text { study }\end{array}$ & 2 \\
\hline $\begin{array}{l}\text { Aves, } \\
\text { Casuariiformes }\end{array}$ & $\begin{array}{l}\text { Dromaius } \\
\text { novaehollandiae }\end{array}$ & QMO 11686 & Femur & $\begin{array}{l}\text { GE } \\
\text { BrightSpeed }\end{array}$ & 120 & 80 & 1584 & 0.326 & 0.3 & $\begin{array}{l}\text { This } \\
\text { study }\end{array}$ & 2 \\
\hline $\begin{array}{l}\text { Aves, } \\
\text { Casuariiformes }\end{array}$ & $\begin{array}{l}\text { Dromaius } \\
\text { novaehollandiae }\end{array}$ & QMO 11686 & Tibiotarsus & $\begin{array}{l}\text { GE } \\
\text { BrightSpeed }\end{array}$ & 120 & 55 & 1681 & 0.488 & 0.3 & $\begin{array}{l}\text { This } \\
\text { study }\end{array}$ & 2 \\
\hline $\begin{array}{l}\text { Aves, } \\
\text { Casuariiformes }\end{array}$ & $\begin{array}{l}\text { Dromaius } \\
\text { novaehollandiae }\end{array}$ & QMO 11686 & Fibula & $\begin{array}{l}\text { GE } \\
\text { BrightSpeed }\end{array}$ & 120 & 55 & 1681 & 0.326 & 0.3 & $\begin{array}{l}\text { This } \\
\text { study }\end{array}$ & 2 \\
\hline $\begin{array}{l}\text { Aves, } \\
\text { Casuariiformes }\end{array}$ & $\begin{array}{l}\text { Dromaius } \\
\text { novaehollandiae }\end{array}$ & QMO 16140 & Femur & $\begin{array}{l}\text { GE } \\
\text { BrightSpeed }\end{array}$ & 120 & 55 & 1681 & 0.232 & 0.3 & $\begin{array}{l}\text { This } \\
\text { study }\end{array}$ & 2 \\
\hline $\begin{array}{l}\text { Aves, } \\
\text { Casuariiformes }\end{array}$ & $\begin{array}{l}\text { Dromaius } \\
\text { novaehollandiae }\end{array}$ & QMO 16140 & Tibiotarsus & $\begin{array}{l}\text { GE } \\
\text { BrightSpeed }\end{array}$ & 120 & 55 & 1681 & 0.232 & 0.3 & $\begin{array}{l}\text { This } \\
\text { study }\end{array}$ & 2 \\
\hline
\end{tabular}




\begin{tabular}{|c|c|c|c|c|c|c|c|c|c|c|}
\hline $\begin{array}{l}\text { Aves, } \\
\text { Casuariiformes }\end{array}$ & $\begin{array}{l}\text { Dromaius } \\
\text { novaehollandiae }\end{array}$ & QMO 16140 & Fibula & $\begin{array}{l}\text { GE } \\
\text { BrightSpeed }\end{array}$ & 120 & 55 & 1681 & 0.188 & 0.3 & $\begin{array}{l}\text { This } \\
\text { study }\end{array}$ \\
\hline $\begin{array}{l}\text { Aves, } \\
\text { Casuariiformes }\end{array}$ & $\begin{array}{l}\text { Casuarius } \\
\text { casuarius }\end{array}$ & QMO 30105 & Femur & $\begin{array}{l}\text { GE } \\
\text { BrightSpeed }\end{array}$ & 120 & 55 & 1681 & 0.215 & 0.3 & $\begin{array}{l}\text { This } \\
\text { study }\end{array}$ \\
\hline $\begin{array}{l}\text { Aves, } \\
\text { Casuariiformes }\end{array}$ & $\begin{array}{l}\text { Casuarius } \\
\text { casuarius }\end{array}$ & QMO 30105 & Tibiotarsus & $\begin{array}{l}\text { GE } \\
\text { BrightSpeed }\end{array}$ & 120 & 55 & 1681 & 0.219 & 0.3 & $\begin{array}{l}\text { This } \\
\text { study }\end{array}$ \\
\hline $\begin{array}{l}\text { Aves, } \\
\text { Casuariiformes }\end{array}$ & $\begin{array}{l}\text { Casuarius } \\
\text { casuarius }\end{array}$ & QMO 30105 & Fibula & $\begin{array}{l}\text { GE } \\
\text { BrightSpeed }\end{array}$ & 120 & 55 & 1681 & 0.215 & 0.3 & $\begin{array}{l}\text { This } \\
\text { study }\end{array}$ \\
\hline $\begin{array}{l}\text { Aves, } \\
\text { Casuariiformes }\end{array}$ & $\begin{array}{l}\text { Casuarius } \\
\text { casuarius }\end{array}$ & QMO 30604 & Femur & $\begin{array}{l}\text { GE } \\
\text { BrightSpeed }\end{array}$ & 120 & 80 & 1584 & 0.467 & 0.3 & $\begin{array}{l}\text { This } \\
\text { study }\end{array}$ \\
\hline $\begin{array}{l}\text { Aves, } \\
\text { Casuariiformes }\end{array}$ & $\begin{array}{l}\text { Casuarius } \\
\text { casuarius }\end{array}$ & QMO 30604 & Tibiotarsus & $\begin{array}{l}\text { GE } \\
\text { BrightSpeed }\end{array}$ & 120 & 80 & 1584 & 0.488 & 0.3 & $\begin{array}{l}\text { This } \\
\text { study }\end{array}$ \\
\hline $\begin{array}{l}\text { Aves, } \\
\text { Casuariiformes }\end{array}$ & $\begin{array}{l}\text { Casuarius } \\
\text { casuarius }\end{array}$ & QMO 30604 & Fibula & $\begin{array}{l}\text { GE } \\
\text { BrightSpeed }\end{array}$ & 120 & 80 & 1584 & 0.488 & 0.3 & $\begin{array}{l}\text { This } \\
\text { study }\end{array}$ \\
\hline $\begin{array}{l}\text { Aves, } \\
\text { Casuariiformes }\end{array}$ & $\begin{array}{l}\text { Casuarius } \\
\text { casuarius }\end{array}$ & QMO 31137 & Femur & $\begin{array}{l}\text { GE } \\
\text { BrightSpeed }\end{array}$ & 120 & 55 & 1681 & 0.213 & 0.3 & $\begin{array}{l}\text { This } \\
\text { study }\end{array}$ \\
\hline
\end{tabular}

Table 1 (continued).

\begin{tabular}{|c|c|c|c|c|c|c|c|c|c|c|c|}
\hline \multirow[b]{2}{*}{$\begin{array}{c}\text { Higher-order } \\
\text { taxonomy }\end{array}$} & \multirow[b]{2}{*}{ Species } & \multirow[b]{2}{*}{$\begin{array}{l}\text { Specimen } \\
\text { number* }\end{array}$} & \multirow[b]{2}{*}{ Element } & \multicolumn{6}{|c|}{ CT scan settings } & \multirow[b]{2}{*}{ Study } & \multirow[b]{2}{*}{$\begin{array}{c}\text { Image } \\
\text { processing } \\
\text { protocol }\end{array}$} \\
\hline & & & & Machine & $\begin{array}{c}\text { Peak } \\
\text { tube } \\
\text { voltage } \\
(\mathrm{kV})\end{array}$ & $\begin{array}{c}\text { Tube } \\
\text { current } \\
(\mathbf{m A})\end{array}$ & $\begin{array}{l}\text { Exposur } \\
\text { e time } \\
(\mathrm{ms})\end{array}$ & $\begin{array}{l}\text { In-plane } \\
\text { pixel } \\
\text { resolution } \\
(\mathrm{mm})\end{array}$ & $\begin{array}{c}\text { Slice } \\
\text { thicknes } \\
\text { s (mm) }\end{array}$ & & \\
\hline $\begin{array}{l}\text { Aves, } \\
\text { Casuariiformes }\end{array}$ & $\begin{array}{l}\text { Casuarius } \\
\text { casuarius }\end{array}$ & $\begin{array}{l}\text { QMO } \\
31137\end{array}$ & Tibiotarsus & $\begin{array}{l}\text { GE } \\
\text { BrightSpe } \\
\text { ed }\end{array}$ & 120 & 80 & 1584 & 0.488 & 0.3 & $\begin{array}{l}\text { This } \\
\text { study }\end{array}$ & 2 \\
\hline $\begin{array}{l}\text { Aves, } \\
\text { Casuariiformes }\end{array}$ & $\begin{array}{l}\text { Casuarius } \\
\text { casuarius }\end{array}$ & $\begin{array}{l}\text { QMO } \\
31137\end{array}$ & Fibula & $\begin{array}{l}\text { GE } \\
\text { BrightSpe } \\
\text { ed }\end{array}$ & 120 & 80 & 1584 & 0.488 & 0.3 & $\begin{array}{l}\text { This } \\
\text { study }\end{array}$ & 2 \\
\hline $\begin{array}{l}\text { Aves, } \\
\text { Casuariiformes }\end{array}$ & $\begin{array}{l}\text { Dromaius } \\
\text { novaehollandia } \\
e\end{array}$ & YPM 2128 & Femur & $\begin{array}{l}\text { GE } \\
\text { Lightspeed } \\
16\end{array}$ & 100 & 70 & 1297 & 0.188 & 0.5 & $\begin{array}{l}\text { Farke } \\
\text { and } \\
\text { Alicea } \\
\text { (2009) }\end{array}$ & none** \\
\hline
\end{tabular}




\begin{tabular}{|c|c|c|c|c|c|c|c|c|c|c|c|}
\hline $\begin{array}{l}\text { Aves, } \\
\text { Galliformes }\end{array}$ & $\begin{array}{l}\text { Alectura } \\
\text { lathami }\end{array}$ & PJB & Femur & $\begin{array}{l}\text { Siemens } \\
\text { Inveon }\end{array}$ & 80 & 0.45 & 1000 & 0.053 & 0.053 & $\begin{array}{l}\text { This } \\
\text { study }\end{array}$ & 1 \\
\hline $\begin{array}{l}\text { Aves, } \\
\text { Galliformes }\end{array}$ & $\begin{array}{l}\text { Alectura } \\
\text { lathami }\end{array}$ & PJB & Tibiotarsus & $\begin{array}{l}\text { Siemens } \\
\text { Inveon }\end{array}$ & 80 & 0.45 & 1000 & 0.053 & 0.053 & $\begin{array}{l}\text { This } \\
\text { study }\end{array}$ & 1 \\
\hline $\begin{array}{l}\text { Aves, } \\
\text { Galliformes }\end{array}$ & $\begin{array}{l}\text { Alectura } \\
\text { lathami }\end{array}$ & PJB & Fibula & $\begin{array}{l}\text { Siemens } \\
\text { Inveon }\end{array}$ & 80 & 0.45 & 1000 & 0.053 & 0.053 & $\begin{array}{l}\text { This } \\
\text { study }\end{array}$ & 1 \\
\hline $\begin{array}{l}\text { Aves, } \\
\text { Galliformes }\end{array}$ & Leipoa ocellata & $\begin{array}{l}\text { MVB } \\
20194\end{array}$ & Femur & $\begin{array}{l}\text { Siemens } \\
\text { Inveon }\end{array}$ & 80 & 0.45 & 1000 & 0.053 & 0.053 & $\begin{array}{l}\text { This } \\
\text { study }\end{array}$ & 1 \\
\hline $\begin{array}{l}\text { Aves, } \\
\text { Galliformes }\end{array}$ & Leipoa ocellata & $\begin{array}{l}\text { MVB } \\
20194\end{array}$ & Tibiotarsus & $\begin{array}{l}\text { Siemens } \\
\text { Inveon }\end{array}$ & 80 & 0.45 & 1000 & 0.053 & 0.053 & $\begin{array}{l}\text { This } \\
\text { study }\end{array}$ & 1 \\
\hline $\begin{array}{l}\text { Aves, } \\
\text { Galliformes }\end{array}$ & Leipoa ocellata & $\begin{array}{l}\text { MVB } \\
20194\end{array}$ & Fibula & $\begin{array}{l}\text { Siemens } \\
\text { Inveon }\end{array}$ & 80 & 0.45 & 1000 & 0.053 & 0.053 & $\begin{array}{l}\text { This } \\
\text { study }\end{array}$ & 1 \\
\hline $\begin{array}{l}\text { Aves, } \\
\text { Galliformes }\end{array}$ & $\begin{array}{l}\text { Numida } \\
\text { meleagris }\end{array}$ & PJB & Femur & $\begin{array}{l}\text { Siemens } \\
\text { Inveon }\end{array}$ & 80 & 0.45 & 1000 & 0.053 & 0.053 & $\begin{array}{l}\text { This } \\
\text { study }\end{array}$ & 1 \\
\hline $\begin{array}{l}\text { Aves, } \\
\text { Galliformes }\end{array}$ & $\begin{array}{l}\text { Numida } \\
\text { meleagris }\end{array}$ & PJB & Tibiotarsus & $\begin{array}{l}\text { Siemens } \\
\text { Inveon }\end{array}$ & 80 & 0.45 & 1000 & 0.053 & 0.053 & $\begin{array}{l}\text { This } \\
\text { study }\end{array}$ & 1 \\
\hline $\begin{array}{l}\text { Aves, } \\
\text { Galliformes }\end{array}$ & $\begin{array}{l}\text { Numida } \\
\text { meleagris }\end{array}$ & PJB & Fibula & $\begin{array}{l}\text { Siemens } \\
\text { Inveon }\end{array}$ & 80 & 0.45 & 1000 & 0.053 & 0.053 & $\begin{array}{l}\text { This } \\
\text { study }\end{array}$ & 1 \\
\hline $\begin{array}{l}\text { Aves, } \\
\text { Galliformes }\end{array}$ & $\begin{array}{l}\text { Colinus } \\
\text { virginianus }\end{array}$ & PJB & Femur & $\begin{array}{l}\text { Siemens } \\
\text { Inveon }\end{array}$ & 80 & 0.35 & 1400 & 0.035 & 0.035 & $\begin{array}{l}\text { This } \\
\text { study }\end{array}$ & 1 \\
\hline $\begin{array}{l}\text { Aves, } \\
\text { Galliformes }\end{array}$ & $\begin{array}{l}\text { Colinus } \\
\text { virginianus }\end{array}$ & PJB & Tibiotarsus & $\begin{array}{l}\text { Siemens } \\
\text { Inveon }\end{array}$ & 80 & 0.45 & 1000 & 0.053 & 0.053 & $\begin{array}{l}\text { This } \\
\text { study }\end{array}$ & 1 \\
\hline
\end{tabular}

Table 1 (continued).

\begin{tabular}{|c|c|c|c|c|c|c|c|c|c|c|c|}
\hline \multirow[b]{2}{*}{$\begin{array}{l}\text { Higher-order } \\
\text { taxonomy }\end{array}$} & \multirow[b]{2}{*}{ Species } & \multirow[b]{2}{*}{$\begin{array}{l}\text { Specimen } \\
\text { number* }\end{array}$} & \multirow[b]{2}{*}{ Element } & \multicolumn{6}{|c|}{ CT scan settings } & \multirow[b]{2}{*}{ Study } & \multirow[b]{2}{*}{$\begin{array}{c}\text { Image } \\
\text { processing } \\
\text { protocol }\end{array}$} \\
\hline & & & & Machine & $\begin{array}{c}\text { Peak } \\
\text { tube } \\
\text { voltage } \\
(\mathrm{kV})\end{array}$ & $\begin{array}{c}\text { Tube } \\
\text { current } \\
\text { (mA) }\end{array}$ & $\begin{array}{c}\text { Exposur } \\
\text { e time } \\
(\mathrm{ms})\end{array}$ & $\begin{array}{c}\text { In-plane } \\
\text { pixel } \\
\text { resolution } \\
(\mathrm{mm})\end{array}$ & $\begin{array}{c}\text { Slice } \\
\text { thicknes } \\
\text { s (mm) }\end{array}$ & & \\
\hline $\begin{array}{l}\text { Aves, } \\
\text { Galliformes }\end{array}$ & $\begin{array}{l}\text { Colinus } \\
\text { virginianus }\end{array}$ & PJB & Fibula & $\begin{array}{l}\text { Siemens } \\
\text { Inveon }\end{array}$ & 80 & 0.45 & 1000 & 0.053 & 0.053 & $\begin{array}{l}\text { This } \\
\text { study }\end{array}$ & 1 \\
\hline $\begin{array}{l}\text { Aves, } \\
\text { Galliformes }\end{array}$ & $\begin{array}{l}\text { Coturnix } \\
\text { chinensis }\end{array}$ & PJB & Femur & $\begin{array}{l}\text { Siemens } \\
\text { Inveon }\end{array}$ & 80 & 0.35 & 1400 & 0.035 & 0.035 & $\begin{array}{l}\text { This } \\
\text { study }\end{array}$ & 1 \\
\hline
\end{tabular}




\begin{tabular}{|c|c|c|c|c|c|c|c|c|c|c|c|}
\hline $\begin{array}{l}\text { Aves, } \\
\text { Galliformes }\end{array}$ & $\begin{array}{l}\text { Coturnix } \\
\text { chinensis }\end{array}$ & PJB & Tibiotarsus & $\begin{array}{l}\text { Siemens } \\
\text { Inveon }\end{array}$ & 80 & 0.35 & 1400 & 0.035 & 0.035 & $\begin{array}{l}\text { This } \\
\text { study }\end{array}$ & 1 \\
\hline $\begin{array}{l}\text { Aves, } \\
\text { Galliformes }\end{array}$ & $\begin{array}{l}\text { Coturnix } \\
\text { chinensis }\end{array}$ & PJB & Fibula & $\begin{array}{l}\text { Siemens } \\
\text { Inveon }\end{array}$ & 80 & 0.35 & 1400 & 0.035 & 0.035 & $\begin{array}{l}\text { This } \\
\text { study }\end{array}$ & 1 \\
\hline $\begin{array}{l}\text { Aves, } \\
\text { Galliformes }\end{array}$ & $\begin{array}{l}\text { Coturnix } \\
\text { japonica }\end{array}$ & PJB & Femur & $\begin{array}{l}\text { Siemens } \\
\text { Inveon }\end{array}$ & 80 & 0.35 & 1400 & 0.035 & 0.035 & $\begin{array}{l}\text { This } \\
\text { study }\end{array}$ & 1 \\
\hline $\begin{array}{l}\text { Aves, } \\
\text { Galliformes }\end{array}$ & $\begin{array}{l}\text { Coturnix } \\
\text { japonica }\end{array}$ & PJB & Tibiotarsus & $\begin{array}{l}\text { Siemens } \\
\text { Inveon }\end{array}$ & 80 & 0.45 & 1000 & 0.053 & 0.053 & $\begin{array}{l}\text { This } \\
\text { study }\end{array}$ & 1 \\
\hline $\begin{array}{l}\text { Aves, } \\
\text { Galliformes }\end{array}$ & $\begin{array}{l}\text { Coturnix } \\
\text { japonica }\end{array}$ & PJB & Fibula & $\begin{array}{l}\text { Siemens } \\
\text { Inveon }\end{array}$ & 80 & 0.45 & 1000 & 0.053 & 0.053 & $\begin{array}{l}\text { This } \\
\text { study }\end{array}$ & 1 \\
\hline $\begin{array}{l}\text { Aves, } \\
\text { Galliformes }\end{array}$ & Gallus gallus & PJB & Femur & $\begin{array}{l}\text { Siemens } \\
\text { Inveon }\end{array}$ & 80 & 0.45 & 1000 & 0.053 & 0.053 & $\begin{array}{l}\text { This } \\
\text { study }\end{array}$ & 1 \\
\hline $\begin{array}{l}\text { Aves, } \\
\text { Galliformes }\end{array}$ & Gallus gallus & PJB & Tibiotarsus & $\begin{array}{l}\text { Siemens } \\
\text { Inveon }\end{array}$ & 80 & 0.45 & 1000 & 0.053 & 0.053 & $\begin{array}{l}\text { This } \\
\text { study }\end{array}$ & 1 \\
\hline $\begin{array}{l}\text { Aves, } \\
\text { Galliformes }\end{array}$ & Gallus gallus & PJB & Fibula & $\begin{array}{l}\text { Siemens } \\
\text { Inveon }\end{array}$ & 80 & 0.45 & 1000 & 0.053 & 0.053 & $\begin{array}{l}\text { This } \\
\text { study }\end{array}$ & 1 \\
\hline $\begin{array}{l}\text { Aves, } \\
\text { Galliformes }\end{array}$ & Gallus gallus & PJB & Femur & $\begin{array}{l}\text { Siemens } \\
\text { Inveon }\end{array}$ & 80 & 0.45 & 1000 & 0.053 & 0.053 & $\begin{array}{l}\text { This } \\
\text { study }\end{array}$ & $\begin{array}{l}1 \text { (for head } \\
\text { only) }\end{array}$ \\
\hline $\begin{array}{l}\text { Aves, } \\
\text { Galliformes }\end{array}$ & $\begin{array}{l}\text { Meleagris } \\
\text { gallopavo }\end{array}$ & PJB & Femur & $\begin{array}{l}\text { Siemens } \\
\text { Inveon }\end{array}$ & 80 & 0.45 & 1000 & 0.053 & 0.053 & $\begin{array}{l}\text { This } \\
\text { study }\end{array}$ & 1 \\
\hline $\begin{array}{l}\text { Aves, } \\
\text { Galliformes }\end{array}$ & $\begin{array}{l}\text { Meleagris } \\
\text { gallopavo }\end{array}$ & PJB & Tibiotarsus & $\begin{array}{l}\text { Siemens } \\
\text { Inveon }\end{array}$ & 80 & 0.45 & 1000 & 0.053 & 0.053 & $\begin{array}{l}\text { This } \\
\text { study }\end{array}$ & 1 \\
\hline $\begin{array}{l}\text { Aves, } \\
\text { Galliformes }\end{array}$ & $\begin{array}{l}\text { Meleagris } \\
\text { gallopavo }\end{array}$ & PJB & Fibula & $\begin{array}{l}\text { Siemens } \\
\text { Inveon }\end{array}$ & 80 & 0.45 & 1000 & 0.053 & 0.053 & $\begin{array}{l}\text { This } \\
\text { study }\end{array}$ & 1 \\
\hline $\begin{array}{l}\text { Aves, } \\
\text { Galliformes }\end{array}$ & $\begin{array}{l}\text { Meleagris } \\
\text { gallopavo }\end{array}$ & $\begin{array}{l}\text { RVC } \\
\text { turkey } 1\end{array}$ & Tibiotarsus & $\begin{array}{l}\text { Nikon } \\
\text { HMX ST } \\
225\end{array}$ & & & & 0.122 & 0.122 & $\begin{array}{l}\text { Doube } \\
\text { et al. } \\
(2012)\end{array}$ & 1 \\
\hline
\end{tabular}

Table 1 (continued).

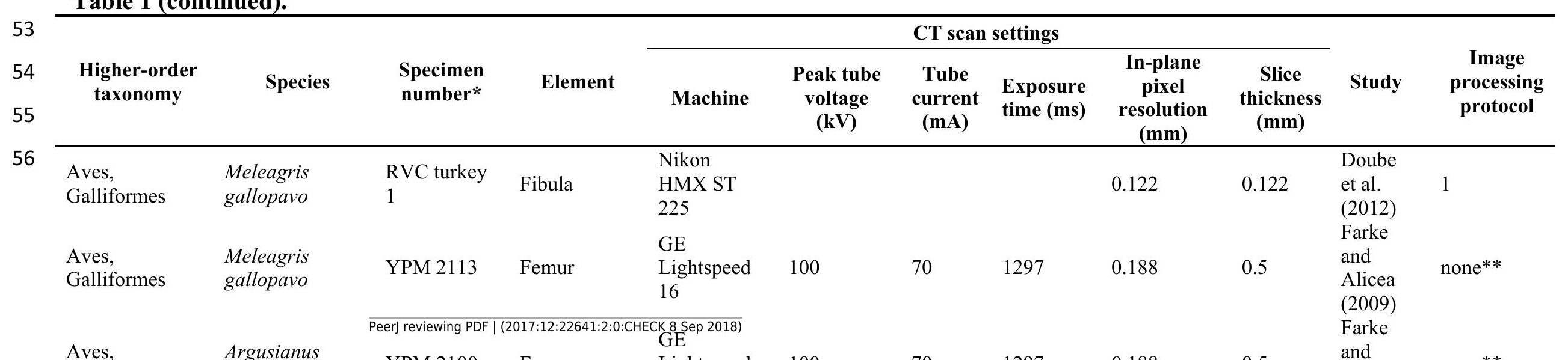


77 Table 1 (continued).

\begin{tabular}{|c|c|c|c|c|c|c|c|c|c|c|c|}
\hline \multirow[b]{2}{*}{$\begin{array}{l}\text { Higher-order } \\
\text { taxonomy }\end{array}$} & \multirow[b]{2}{*}{ Species } & \multirow[b]{2}{*}{$\begin{array}{l}\text { Specimen } \\
\text { number* }\end{array}$} & \multirow[b]{2}{*}{ Element } & \multicolumn{6}{|c|}{ CT scan settings } & \multirow[b]{2}{*}{ Study } & \multirow[b]{2}{*}{$\begin{array}{c}\text { Image } \\
\text { processing } \\
\text { protocol }\end{array}$} \\
\hline & & & & Machine & $\begin{array}{c}\text { Peak } \\
\text { tube } \\
\text { voltage } \\
(\mathbf{k V})\end{array}$ & $\begin{array}{c}\text { Tube } \\
\text { current } \\
\text { (mA) }\end{array}$ & $\begin{array}{l}\text { Exposure } \\
\text { time (ms) }\end{array}$ & $\begin{array}{c}\text { In-plane } \\
\text { pixel } \\
\text { resolution } \\
(\mathbf{m m})\end{array}$ & $\begin{array}{c}\begin{array}{c}\text { Slice } \\
\text { thickness } \\
\text { (mm) }\end{array}\end{array}$ & & \\
\hline $\begin{array}{l}\text { Aves, } \\
\text { Gruiformes }\end{array}$ & $\begin{array}{l}\text { Gallinula } \\
\text { tenebrosa }\end{array}$ & PJB & Tibiotarsus & $\begin{array}{l}\text { Siemens } \\
\text { Inveon }\end{array}$ & 80 & 0.45 & 1000 & 0.053 & 0.053 & This study & 1 \\
\hline $\begin{array}{l}\text { Aves, } \\
\text { Gruiformes }\end{array}$ & $\begin{array}{l}\text { Gallinula } \\
\text { tenebrosa }\end{array}$ & Peerlf Peviewing PDF & 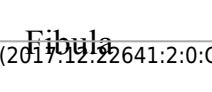 & $\frac{\text { Siemens }}{\text { SEChaseont }}$ & 80 & 0.45 & 1000 & 0.053 & 0.053 & This study & 1 \\
\hline
\end{tabular}


82 


\section{Table 2 (on next page)}

Statistical results of categorical scoring analyses of cancellous bone architecture in bird femora and tibiotarsi versus bone length.

***For features 2 and 3 of the tibiotarsus, an ICC was unable to be calculated, because there was only one specimen in each set that had actually received a numeric score by all five scorers. 
1 Table 2. Statistical results of categorical scoring analyses of cancellous bone architecture in 2 bird femora and tibiotarsi versus bone length.

\begin{tabular}{llccccc}
\hline Element & Feature & Slope & Intercept & $\boldsymbol{r}^{\mathbf{2}}$ & $\boldsymbol{p}$-value & ICC \\
\hline \multirow{3}{*}{ Femur } & 1 (extent) & 0.004123 & 0.3668 & 0.3846 & 0.0002 & 0.8533 \\
& 2 (orientation) & -0.0061 & 6.5355 & 0.2196 & 0.0245 & 0.8064 \\
& 3 (association) & 0.00198 & 0.92015 & 0.1577 & 0.0609 & 0.6909 \\
\multirow{2}{*}{ Tibiotarsus } & 1 (extent) & 0.001268 & 0.14686 & 0.2263 & 0.0168 & 0.4642 \\
& 2 (orientation) & -0.006 & 6.6016 & 0.2807 & 0.1438 & $* * *$ \\
& 3 (association) & 0.001055 & 1.0996 & 0.2239 & 0.2110 & $* * *$ \\
\hline
\end{tabular}

3

4

5

6

7

8

9

10

11

12

13

14

15

16

17

18

19

***For features 2 and 3 of the tibiotarsus, an ICC was unable to be calculated, because there was only one specimen in each set that had actually received a numeric score by all five scorers. 


\section{Figure 1}

Cancellous bone occurrence and macrostructure, as illustrated here with the femur of a cow ( Bos tauros ), sectioned in the coronal plane.

Cancellous bone occurs in the proximal and distal ends of the bone (as indicated by the braces), underlying the thin cortical bone capping the epiphyses and apophyses, as well as the metaphyses. A close-up view of the cancellous bone (right) reveals the high porosity of the tissue, giving it a spongy appearance.

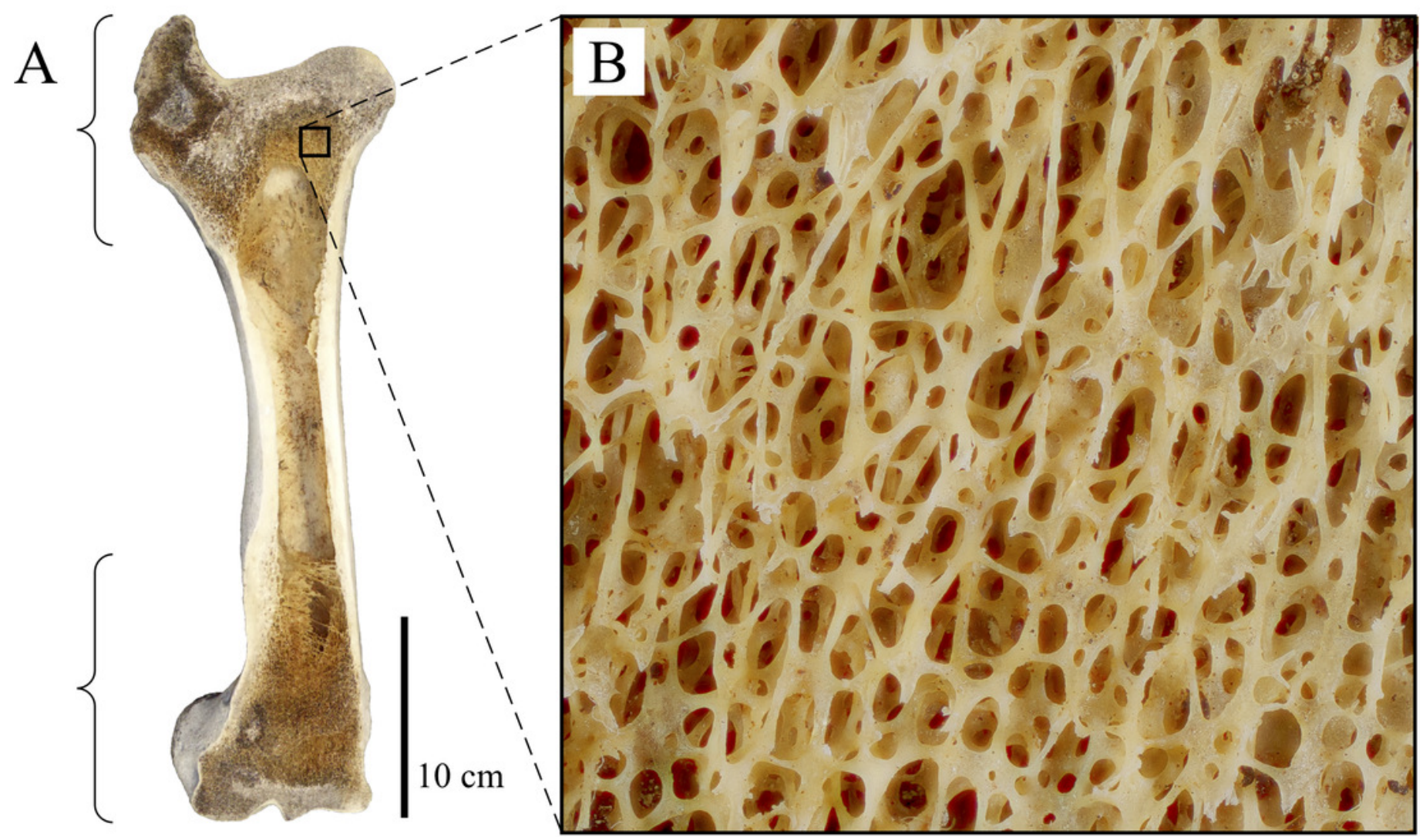




\section{Figure 2}

Cancellous bone fabric as represented by its principal architectural directions.

(A) A cube of cancellous bone of side length $5.33 \mathrm{~mm}$, from the proximal femur of a freshwater crocodile, Crocodylus johnstoni , with the principal directions of the bone's architecture superimposed. As an orthotropic material, cancellous bone fabric is completely described by three principal directions. (B) The fabric ellipsoid representation for this cube of cancellous bone is derived from the vectors that describe the principal architectural directions. The ellipsoid's major, semimajor and minor axes are given by the primary $\left(u_{1}\right)$, secondary $\left(\mathrm{u}_{2}\right)$ and tertiary $\left(\mathrm{u}_{3}\right)$ directions of the cancellous bone architecture, which correspond to the eigenvectors of the fabric tensor. The relative lengths of each axis depend on the relative magnitudes of the principal directions, which correspond to the eigenvalues of the fabric tensor. The degree of anisotropy (DA) describes the extent to which the trabeculae are aligned within a sample, and is given as the relative magnitude of the primary and tertiary eigenvalues (i.e., $\mathrm{DA}=\mathrm{e}_{1} / \mathrm{e}_{3}$ ); in this instance $\mathrm{DA}=1.44$. The cancellous bone geometry was derived via micro-computed X-ray tomographic scanning (Siemens Inveon, 80 $\mathrm{kV}, 500 \mu \mathrm{A}, 900 \mathrm{~ms}$ exposure, $53.3 \mu \mathrm{m}$ isotropic resolution) and 3-D visualization (Mimics 17.0, Materialise NV, Belgium). The material directions were calculated using the mean intercept length method as implemented in the software Quant3D 2.3 (see Ketcham \& Ryan 2004). 

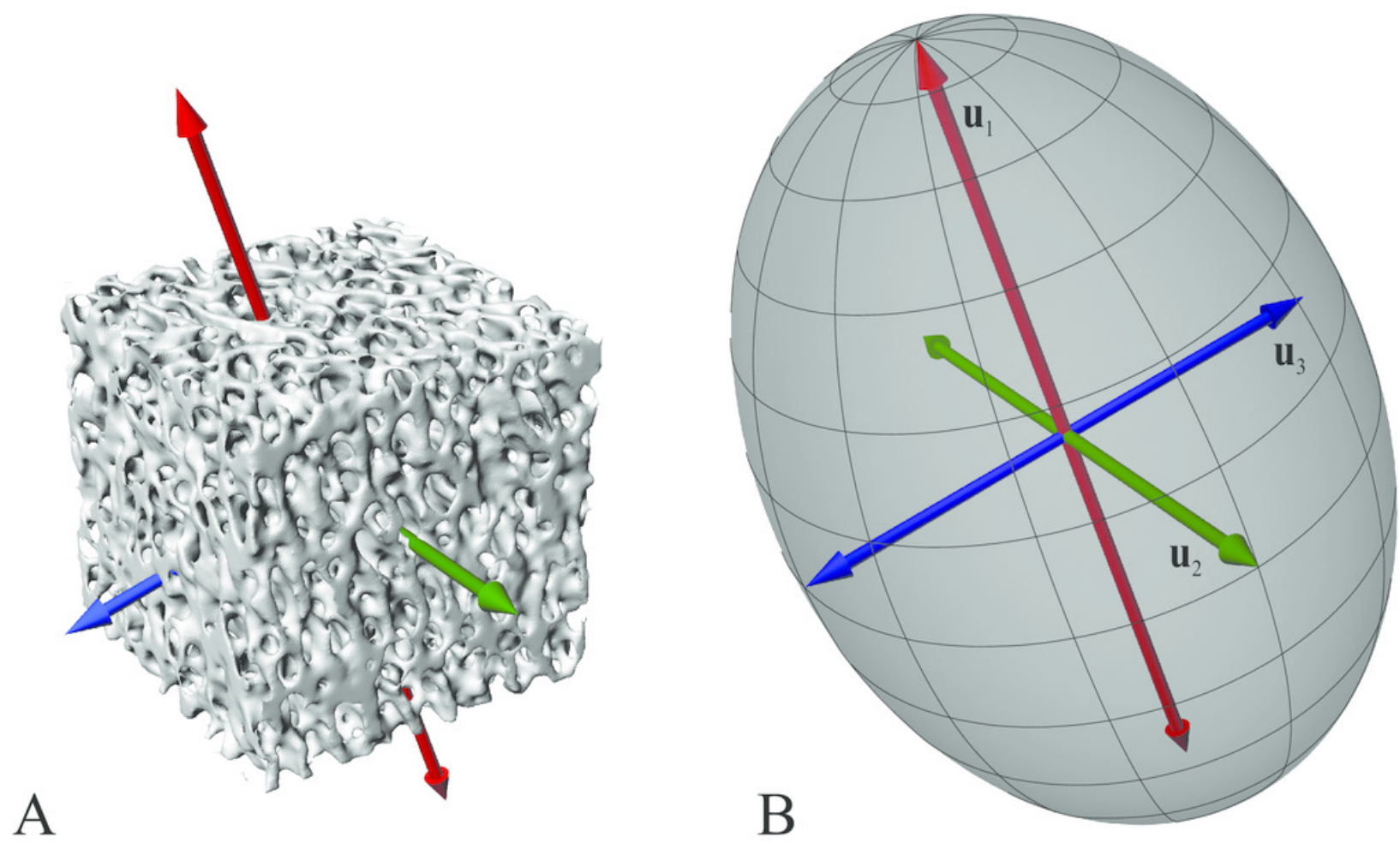


\section{Figure 3}

Cancellous bone fabric direction can change in response to experimentally induced changes in mechanical loading.

(A-C) The study of Goldstein et al. (1991). In the distal femur of normal dogs (A), the principal directions of cancellous bone fabric (arrows) vary throughout the bone. After 38 weeks following surgical implantation of load cells ( $B$, arrows indicate direction of applied principal compressive stress), the principal directions of the cancellous fabric were greatly altered, and were reoriented to align with the compressive stress applied by the load cells (C). (D-F) The study of Pontzer et al. (2006). Subjecting guineafowl to running on inclined treadmills caused them to move with a more flexed knee posture compared to running on the level (the angle $\theta$ is reduced). The postural change resulted in an altered relative orientation of the joint force that the distal femur experienced (E, $F$, red arrow), which after 45 days was found to produce a changed orientation of peak trabecular density (dotted arrow). 


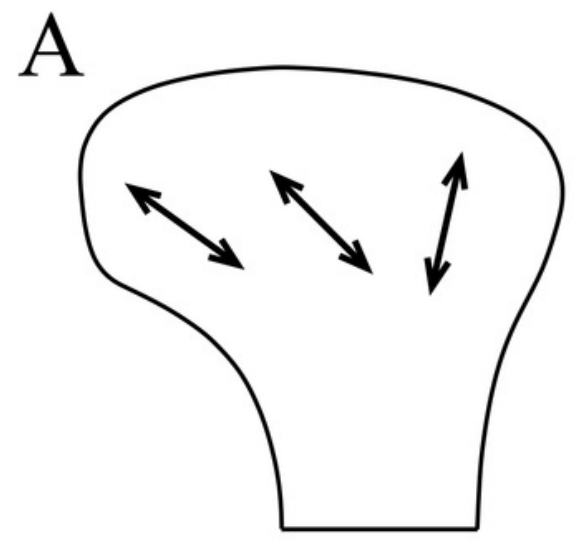

normal (control) femur

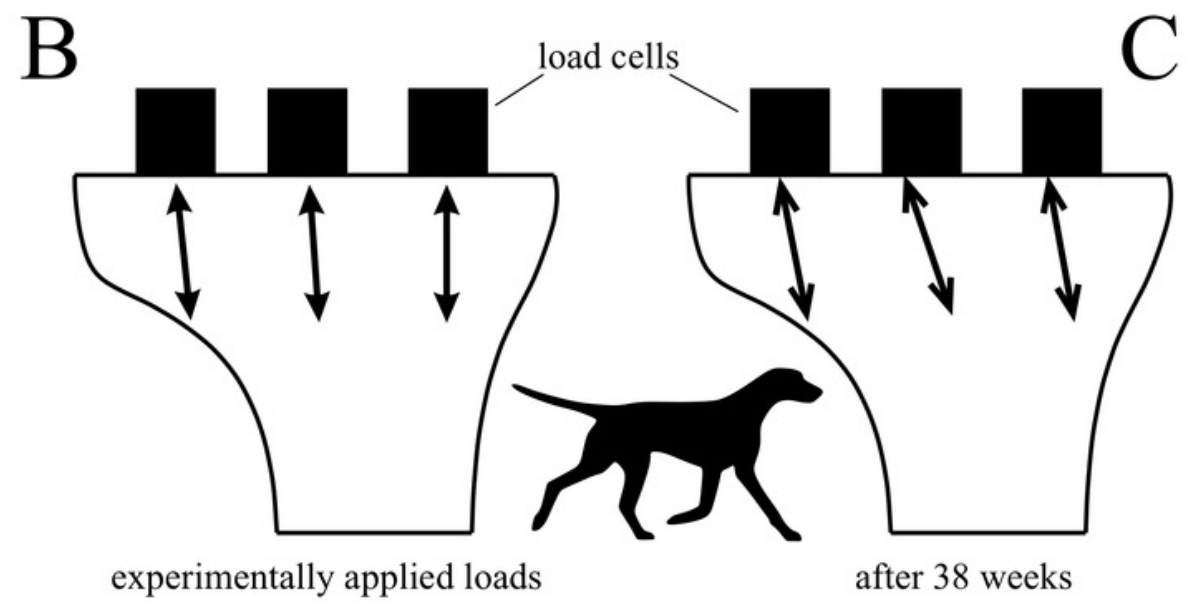

D

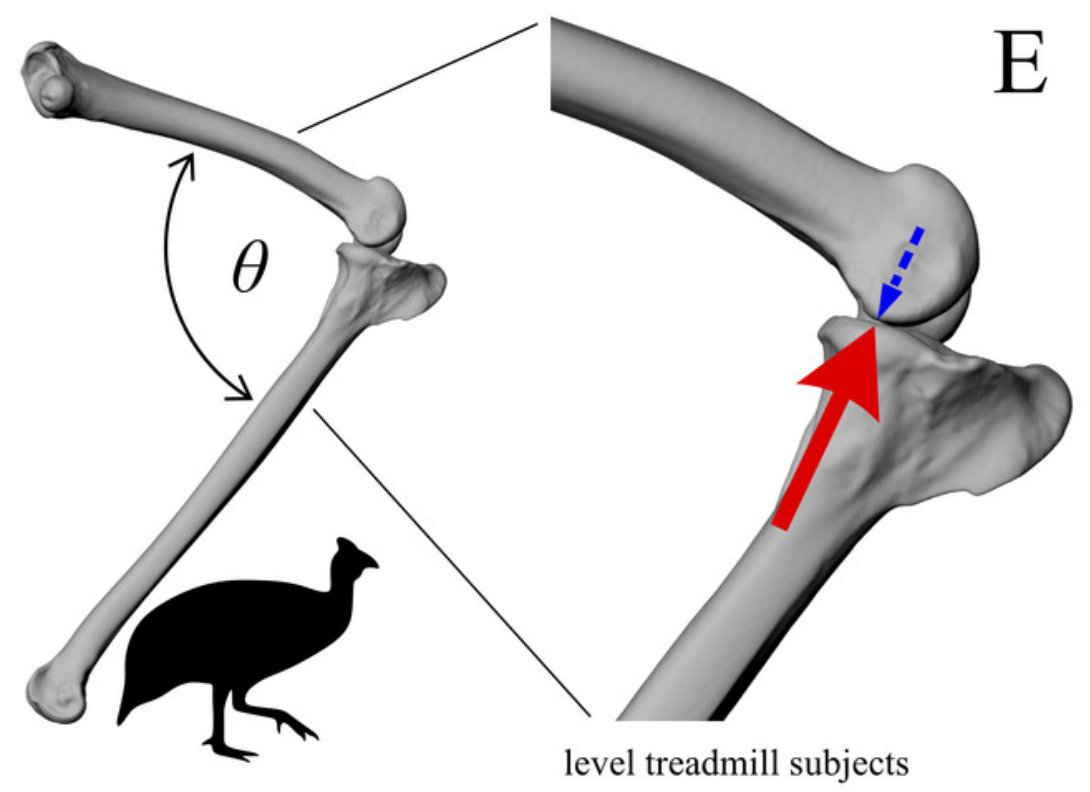

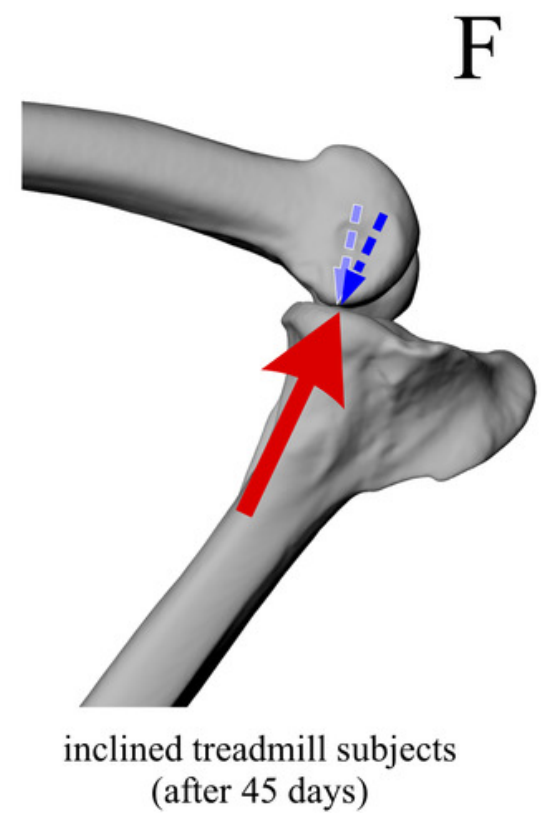

F

(after 45 days) 


\section{Figure 4}

Trabeculae tend to align themselves with the orientation of principal stresses resulting from in vivo loading.

(A) Coronal micro-computed tomographic section through a human proximal femur, illustrating the architecture of cancellous bone. Image provided courtesy of SCANCO Medical AG. (B) A typical loading regime experienced by the proximal femur during locomotion, here the single-legged stance phase of walking (after Rudman et al. 2006). This consists of the joint reaction force applied by the acetabulum (JRF), the force of the adductor muscles pulling on the trochanter (add) and the small forces applied by the capsular ligaments (c). (C) Principal stress trajectories resulting from the loading regime in B, as calculated by a twodimensional finite element analysis (after Rudman et al. 2006). Note the striking correspondence of the main tracts of trabeculae in $\mathrm{A}$ and the principal stress trajectories in $\mathrm{C}$. 

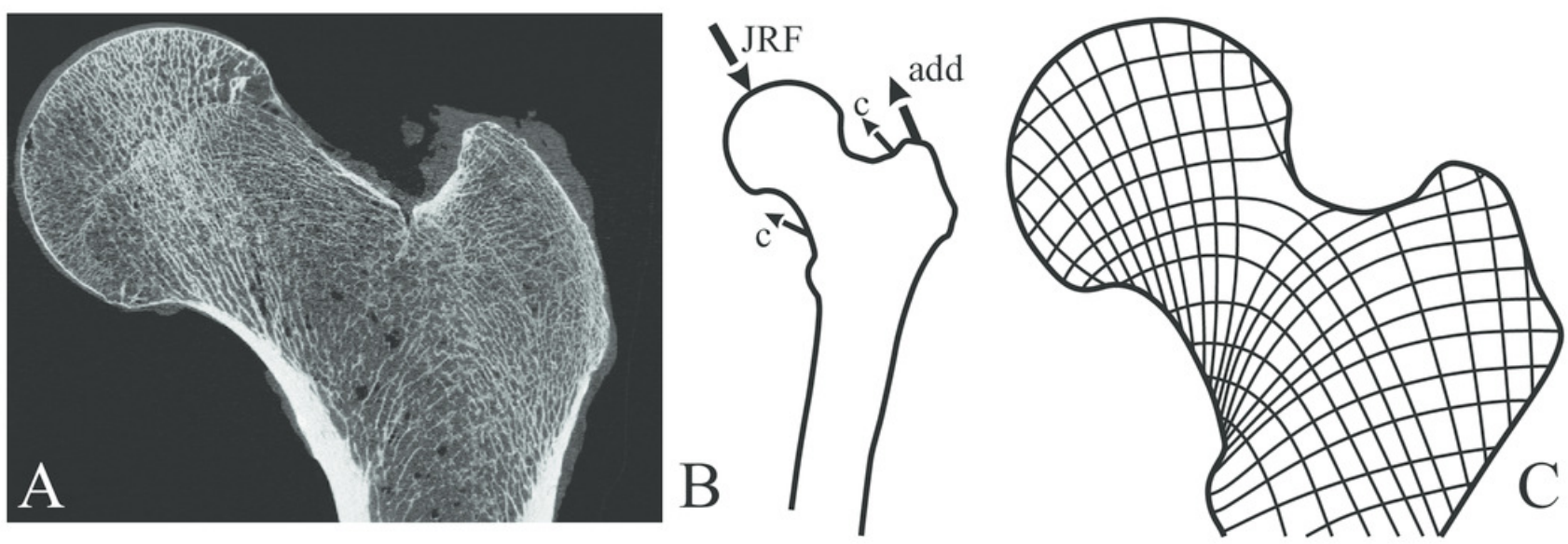


\section{Figure 5}

Cancellous bone remodelling at the cellular level can bring about changes in the entire architecture at the whole-bone level.

(A-D) Schematic illustration of the mechanostat of cancellous bone. Given an initial architecture in A, a change in the loading regime will lead to some parts becoming overloaded (high stress, dotted) and others becoming underloaded (low stress, horizontal hatching) in B. Surface remodelling by osteoblasts and osteoclasts (C) acts to deposit additional bone material in those overloaded areas (dark grey) and remove bone material from those underloaded areas (light grey); arrows show direction in which local bone surface moves. This continues ad infinitum until all bone tissue is neither too highly strained nor too little strained. (E, F) Illustrates the application of the mechanostat principal on the level of the whole bone, via computational modelling (adapted from Jang \& Kim 2008; Jang \& Kim 2010a). In this example of the human proximal femur, with loads simulating both the joint reaction force and forces from the abductor muscles, the initially isotropic architecture (E) undergoes remodelling until equilibrium is reached. The resulting equilibrium architecture (F) is extremely similar to that observed in the real specimen (cf. Fig. 4A). 

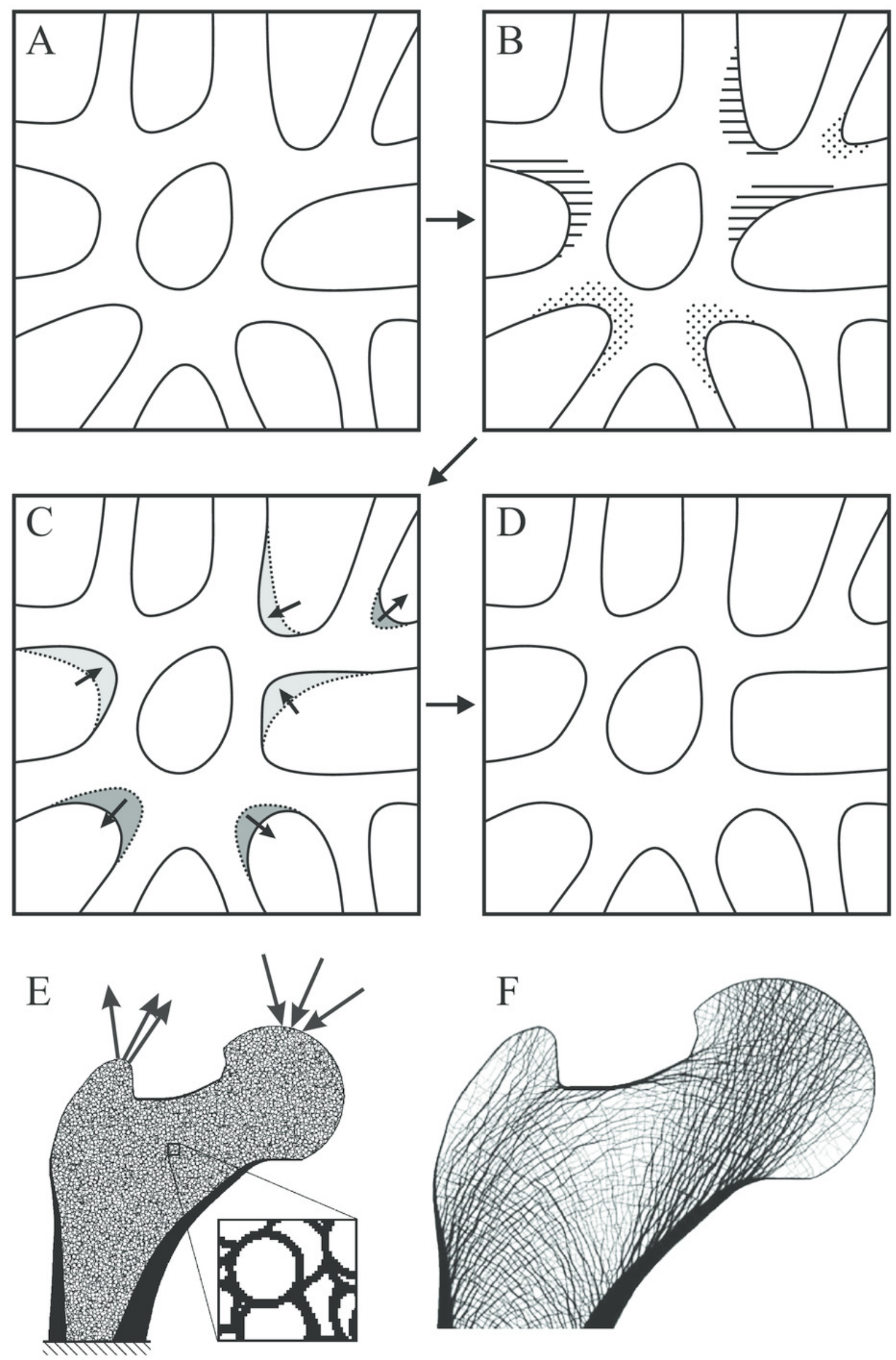


\section{Figure 6}

Orthogonal arrangements of trabeculae usually reflect a highly consistent loading regime experienced by a bone.

(A) Sagittal section through the calcaneum of a cow, with the pull of the Achilles tendon on the distal end indicated by the arrow. (B) A force applied to the free end of a cantilever beam is comparable to the loading regime experienced by the cow calcaneum during locomotion. The bending of the cantilever beam produces principal stress trajectories that are very similar to the overall arrangement of trabeculae in the calcaneum (solid lines are trajectories of compressive stress; dashed lines are trajectories of tensile stress). Since the calcaneum is only loaded in this fashion, the two systems of trabeculae (one curving from up, one curving down) tend to intersect at right angles. 


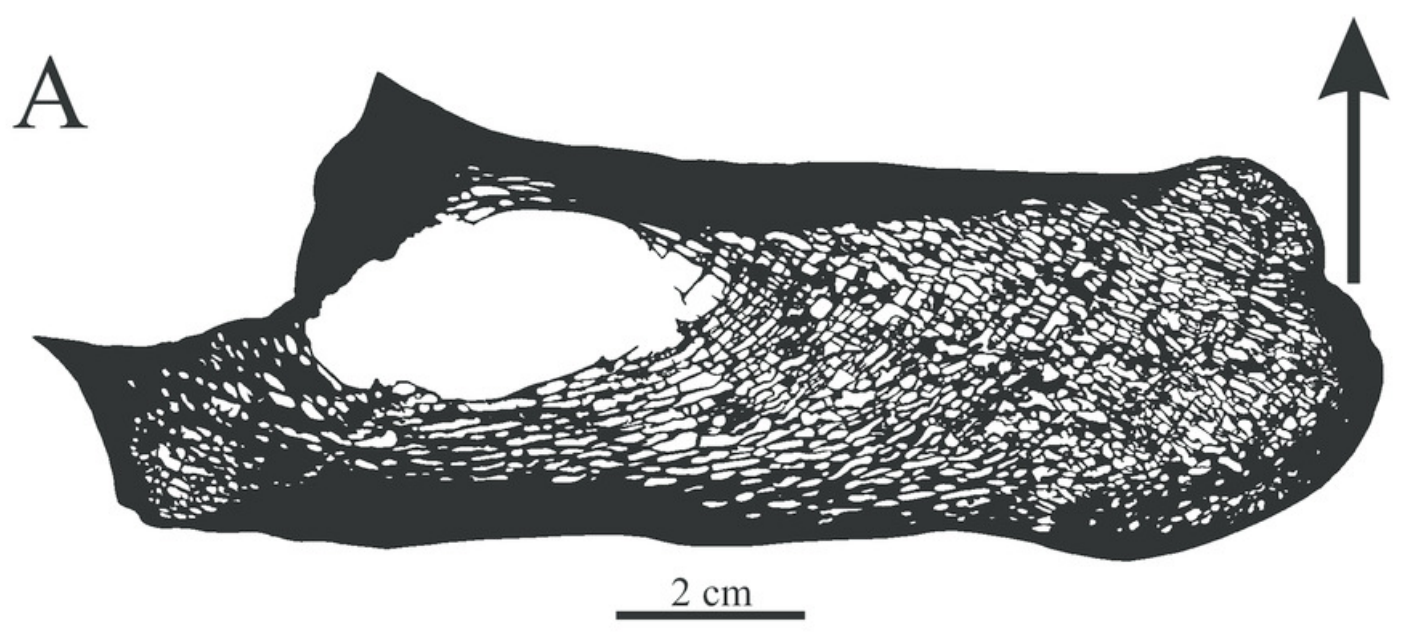

B

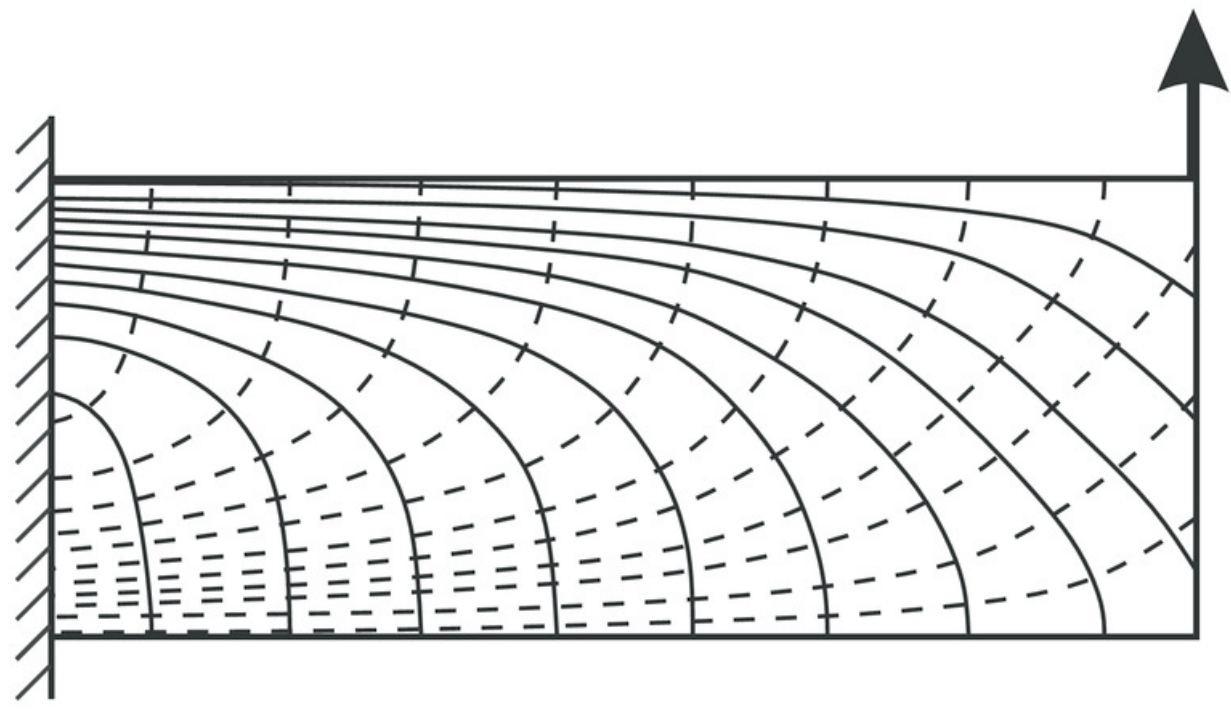




\section{Figure 7 (on next page)}

Three different ways in which the architecture of cancellous bone can be influenced by its mechanical environment.

See main text for full discussion. The dashed grey pathway for epigenetics signifies that it currently remains unknown as to if and how epigenetics may influence cancellous bone mechanobiology. 


\section{i. Locomotion-induced erape lokding}

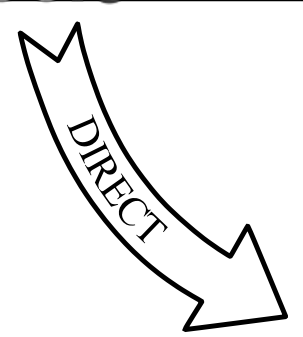

$-$
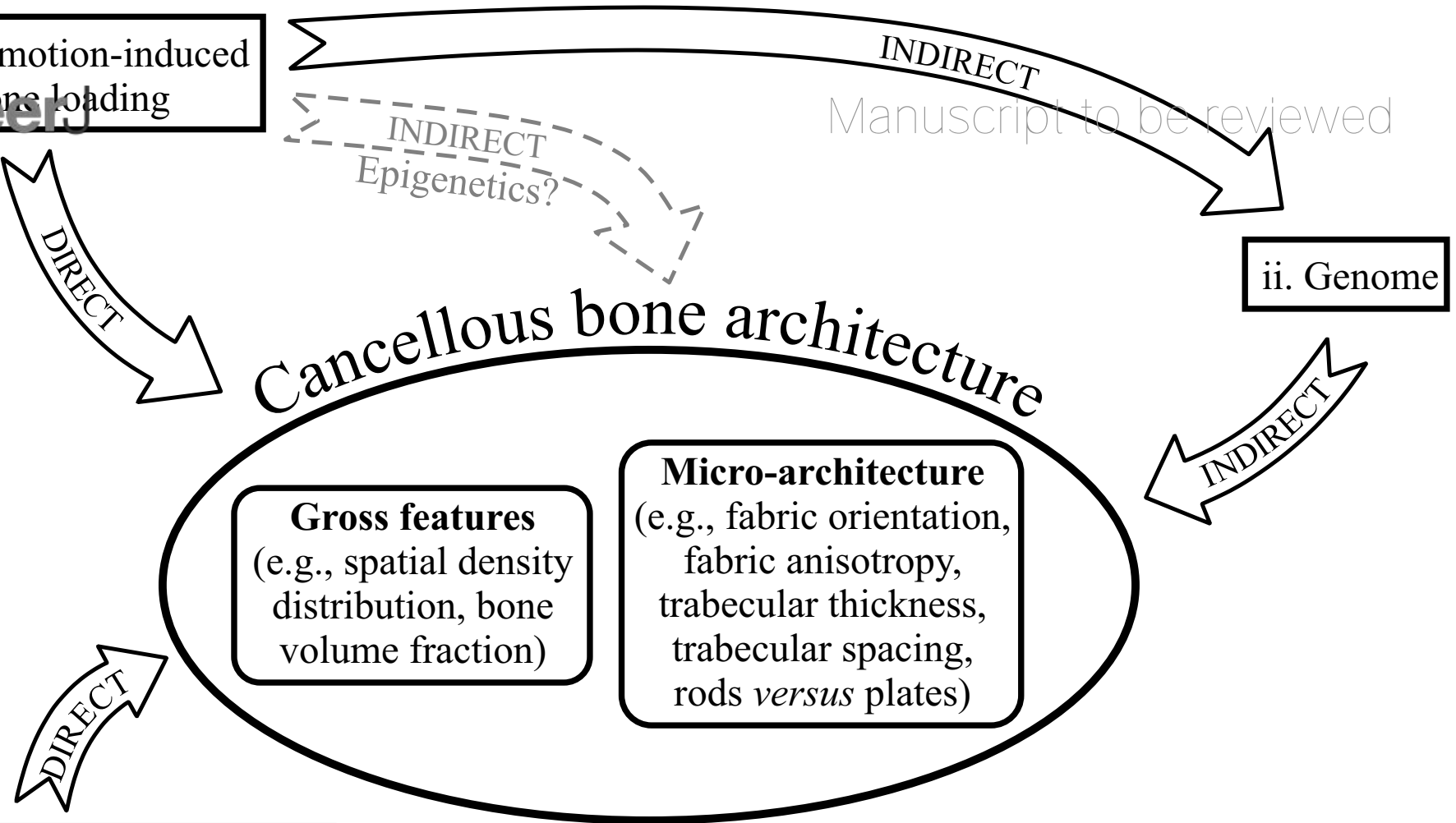

\section{Micro-architecture}

(e.g., fabric orientation, fabric anisotropy, trabecular thickness, trabecular spacing, rods versus plates)

iii. Prenatal bone loading via muscular contractions 


\section{Figure 8 (on next page)}

Phylogenetic interrelationships of the major groups of animals studied.

Interrelationships of non-avian theropods are based on Zanno et al. (2009), Carrano et al. (2012) and Turner et al. (2012); interrelationships of birds are based on Hackett et al. (2008), Morgan-Richards et al. (2008), Ksepka (2009), Phillips et al. (2010), Haddrath \& Baker (2012), Jetz et al. (2012), Smith et al. (2013), Yuri et al. (2013), Mitchell et al. (2014), Jarvis et al. (2014), Ksepka \& Phillips (2015) and Prum et al. (2015). The interrelationships of the neoavian species studied here are currently not well agreed upon, and so Neoaves is shown as an unresolved polytomy. Silhouettes depict exemplar members or each group, and are not to scale. The individual determinate species of non-avian theropod studied are listed in the inset box, and their phylogenetic position signified by superscripts. 


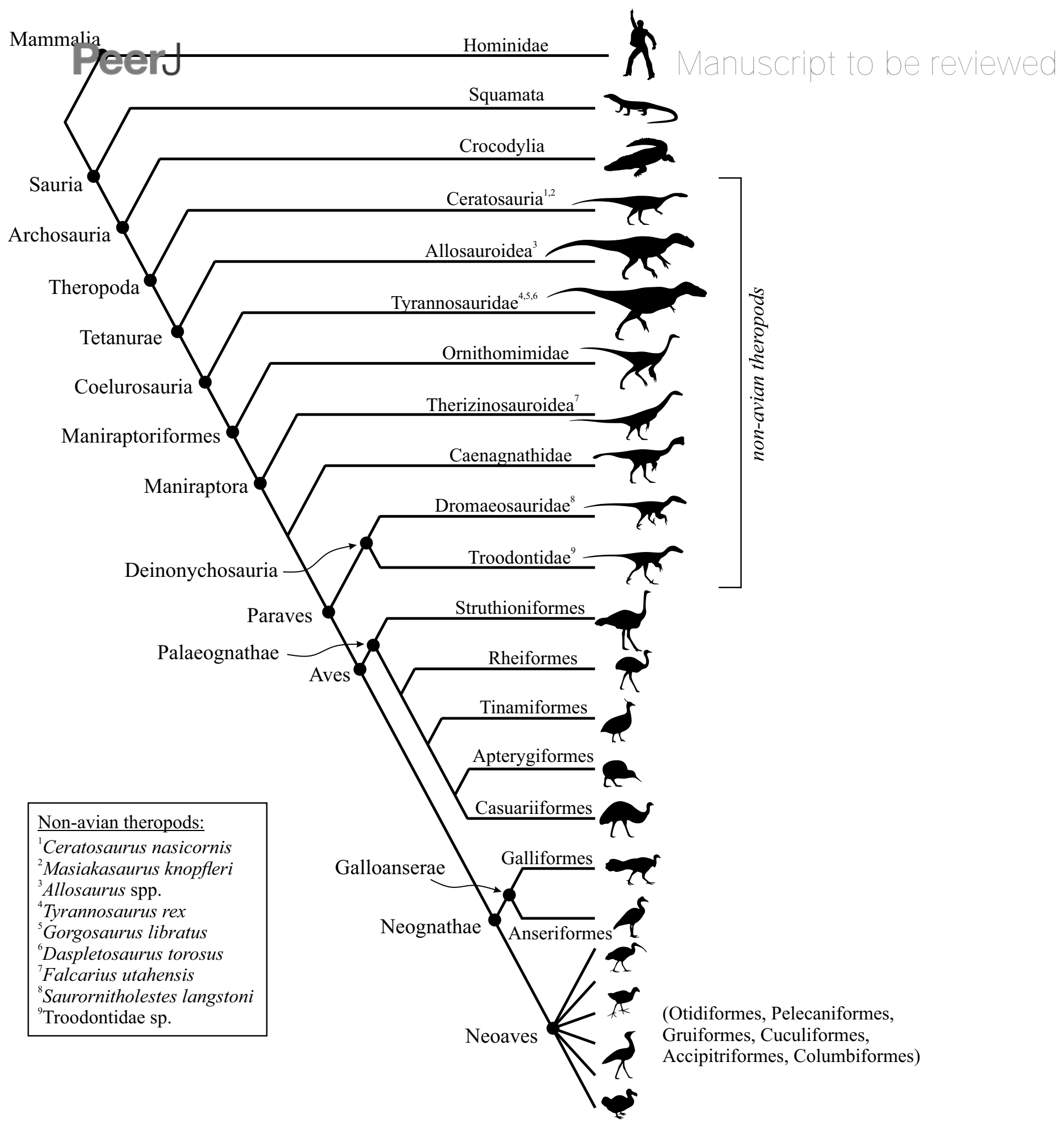




\section{Figure 9}

Resampling the medical CT scans, as per protocol 2, helps produce a more accurate segmentation of the cancellous bone structure.

This is illustrated here with a CT slice of the proximal femur of a southern cassowary, Casuarius casuarius (QMO 31137). (A) The raw CT slice. (B) A local segmentation algorithm, as per protocol 1 , is applied to the slice in A, with window radius 8 pixels and contrast threshold of 25; notice that it performs poorly, with some trabeculae becoming disconnected and some intertrabecular spaces being obliterated. (C) The same CT slice as in A, but resampled to triple the resolution, that is, voxel dimensions are now $1 / 3$ of original in-plane pixel resolution. (D) A local segmentation is applied to the resampled slice in C, using the same parameters as in B; notice that it performs far better in extracting the cancellous structure. Bicubic interpolation results in smoother boundaries to the segmented scans, in contrast to lower-order (e.g., bilinear) interpolation. The location of the insets in each panel is shown in C. 

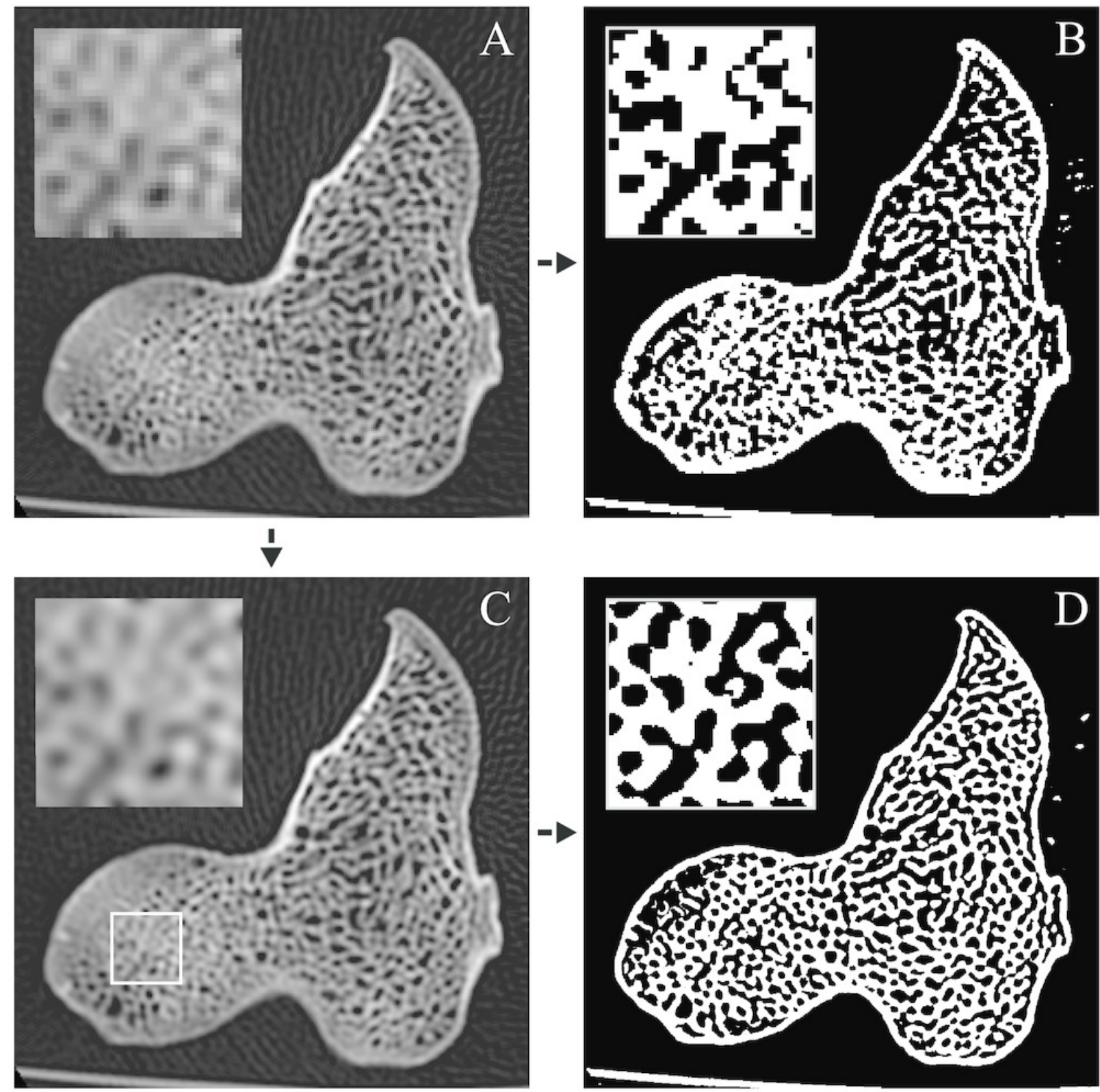


\section{Figure 10}

Schematic illustration of the image processing protocol used for the non-avian theropod micro-CT scans.

(A) The original image, affected by both high and low frequency noise; segmentation of this image by global or local thresholding techniques will not work. (B) A low-radius median filter is applied to remove high-frequency noise. (C) A large-radius median filter is applied to isolate the low-frequency (background) noise. (D) The low-frequency filtered image in C is subtracted from the high-frequency filtered image in B. (E) A low-radius mean filter is applied, followed by a global high-pass segmentation to produce the final image. 


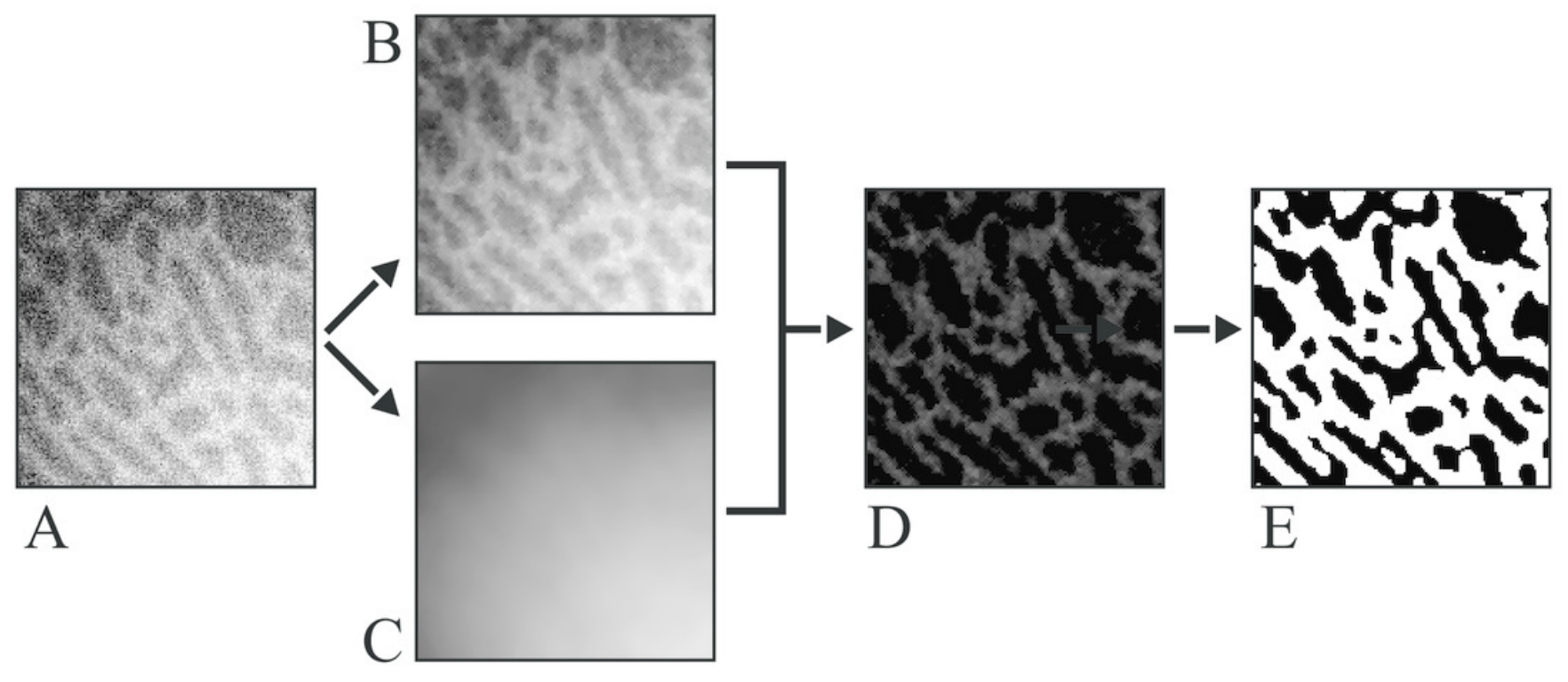




\section{Figure 11}

The arrangement of VOIs for quantitative analysis of cancellous bone architecture follows the geometric pattern of cubic close-packed spheres.

This is illustrated here for the proximal right femur of Troodontidae sp. (MOR 748). Each spherical VOI has a diameter of $5 \mathrm{~mm}$. In this arrangement, each VOI is just touching its neighbouring VOls, and each sphere can be in contact with up to 12 other VOls. (A, B) Shown in medial view. (C, D) Shown in oblique anteromedial view. Notice in C and D that VOI placement avoids the medullary cavity (orange) and cracks (green). 

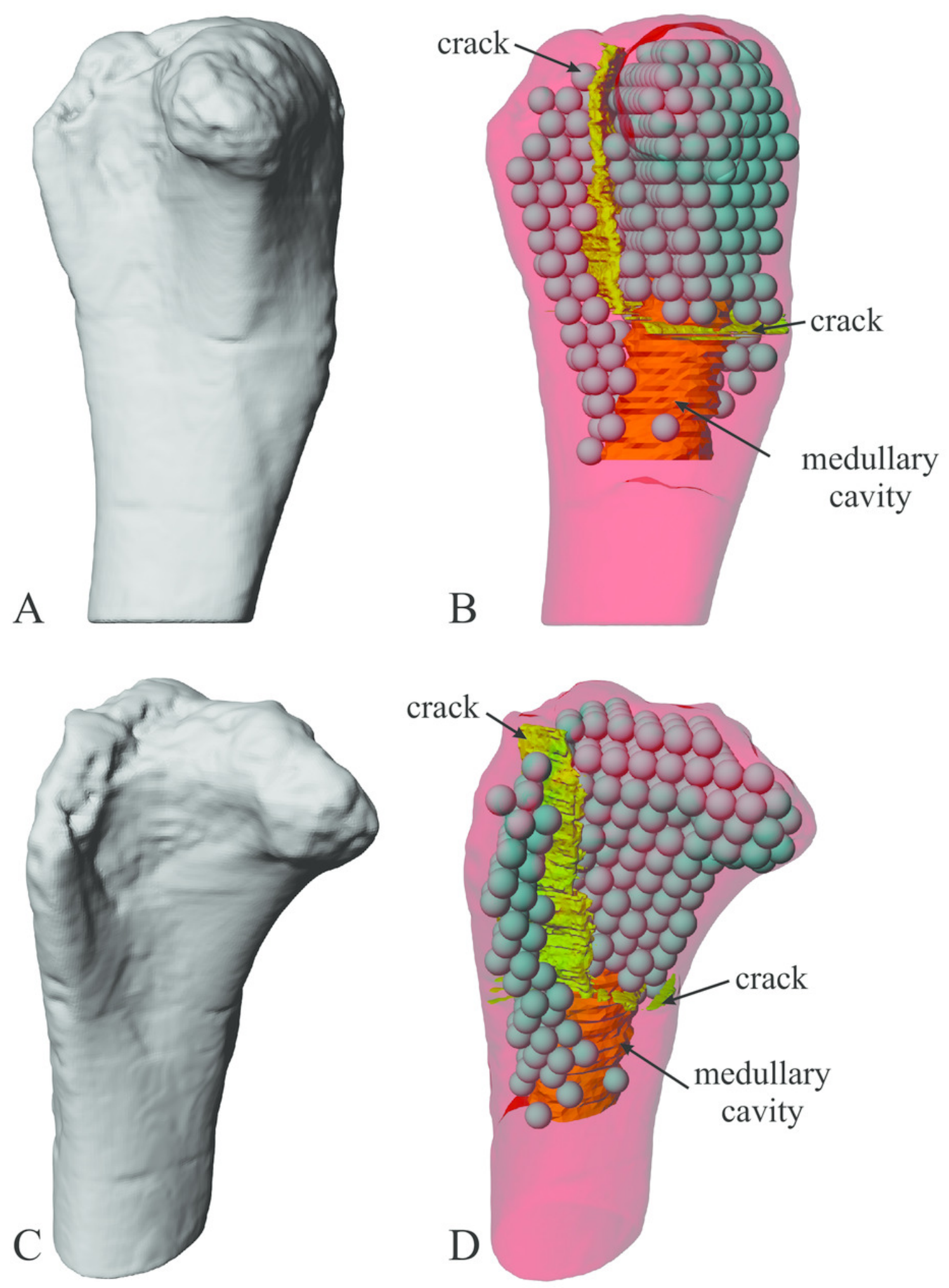


\section{Figure 12}

Definition of the anatomical coordinate system for the femur used in this study.

This is illustrated here with the right femur of a Australian bustard, Ardeotis australis (MVB 20408), in anterior (A) and oblique anteromedial (B) views. The anatomical coordinate system is defined by the principal axis of inertia of the bone (dashed line) and the centres of two spheres fit to the distal condyles (white circles). For left femora, their geometry and calculated mean orientation of the primary fabric vector were mirrored prior to construction of an anatomical coordinate system. 

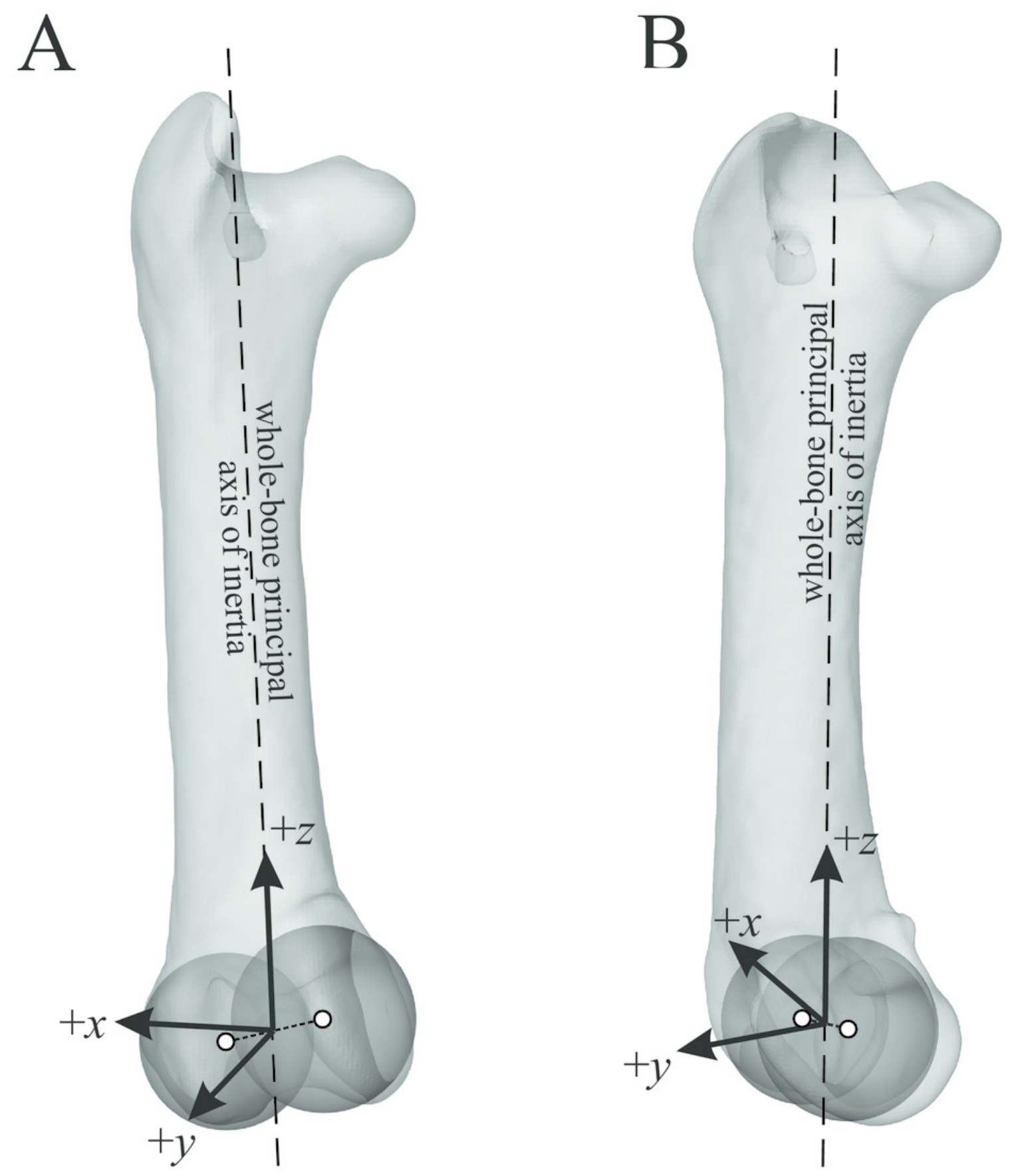


\section{Figure 13}

Schematic illustration of two of the features of diaphyseal cancellous bone that were subject to categorical scoring.

(A) The orientation of trabeculae with respect to the long axis of the shaft. (B) The tendency of trabeculae to be closely associated with other trabeculae. Upper row is oblique view, lower row is section-on view. These illustrations were given to the scorers to provide them with a point of reference. 
A
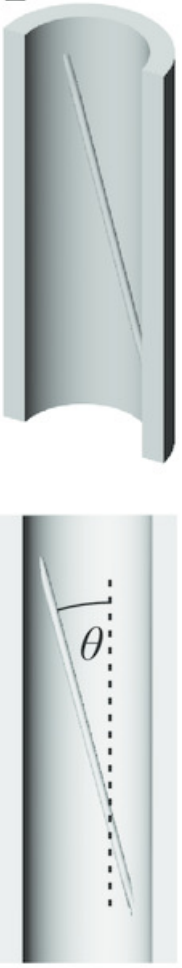

$\theta=15^{\circ}$, score $=1$
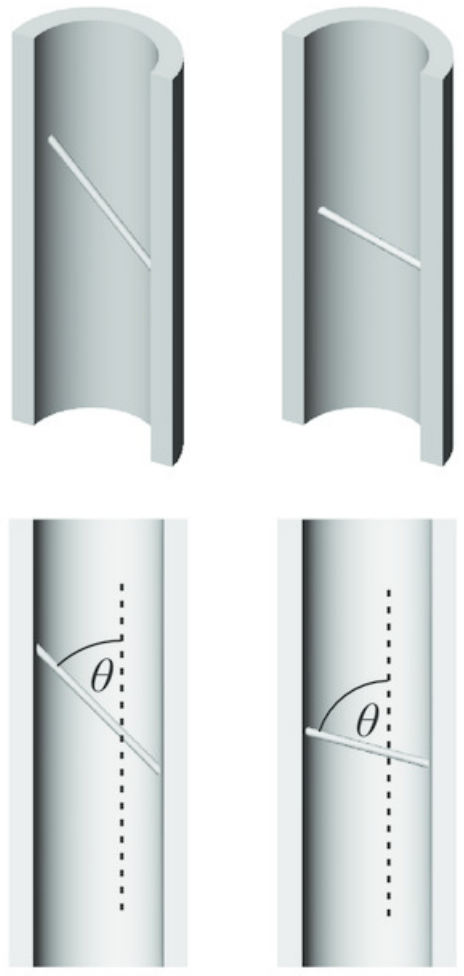

$$
\theta=45^{\circ} \text {, }
$$
score $=4$
B
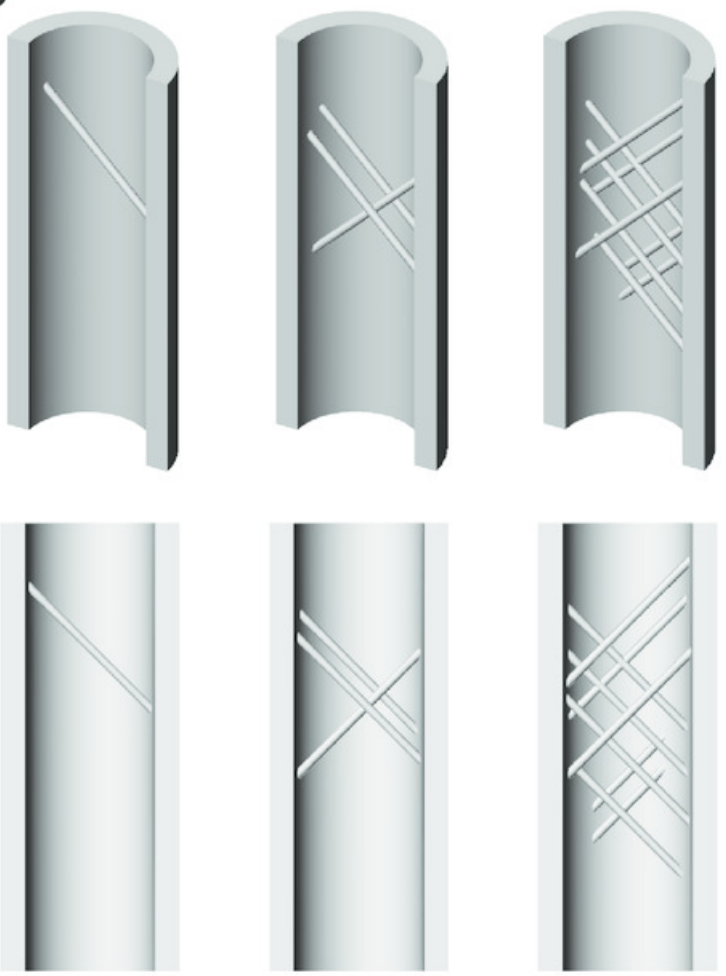

score $=1$

score $=3$ 


\section{Figure 14}

Pneumatization modulates trabecular spacing in the femur of both large and small birds.

This is illustrated here with processed CT scan slices located approximately midway through the femoral head in the axial plane. (A) A marrow-filled femur of a southern cassowary, Casuarius casuarius (QMO 30105), with mean trabecular spacing $=0.638 \mathrm{~mm}$. (B) A pneumatized femur of an emu, Dromaius novaehollandiae (QMO 16140) with mean trabecular spacing $=1.128 \mathrm{~mm}$. (C) A marrow-filled femur of a chicken, Gallus gallus (PJB coll.), with mean trabecular spacing $=0.320 \mathrm{~mm}$. (D) A pneumatized femur of an Australian brush turkey, Alectura lathami (PJB coll.), with mean trabecular spacing $=0.999 \mathrm{~mm}$.

Reported trabecular spacing values were calculated (for illustrative purposes) for the femoral head using the BoneJ 1.3.11 plugin for ImageJ (Doube et al. 2010). A and B are shown at the same scale, as are $C$ and $D$. Scale bars are $10 \mathrm{~mm}$, and yellow asterisks denote pneumatopores. 

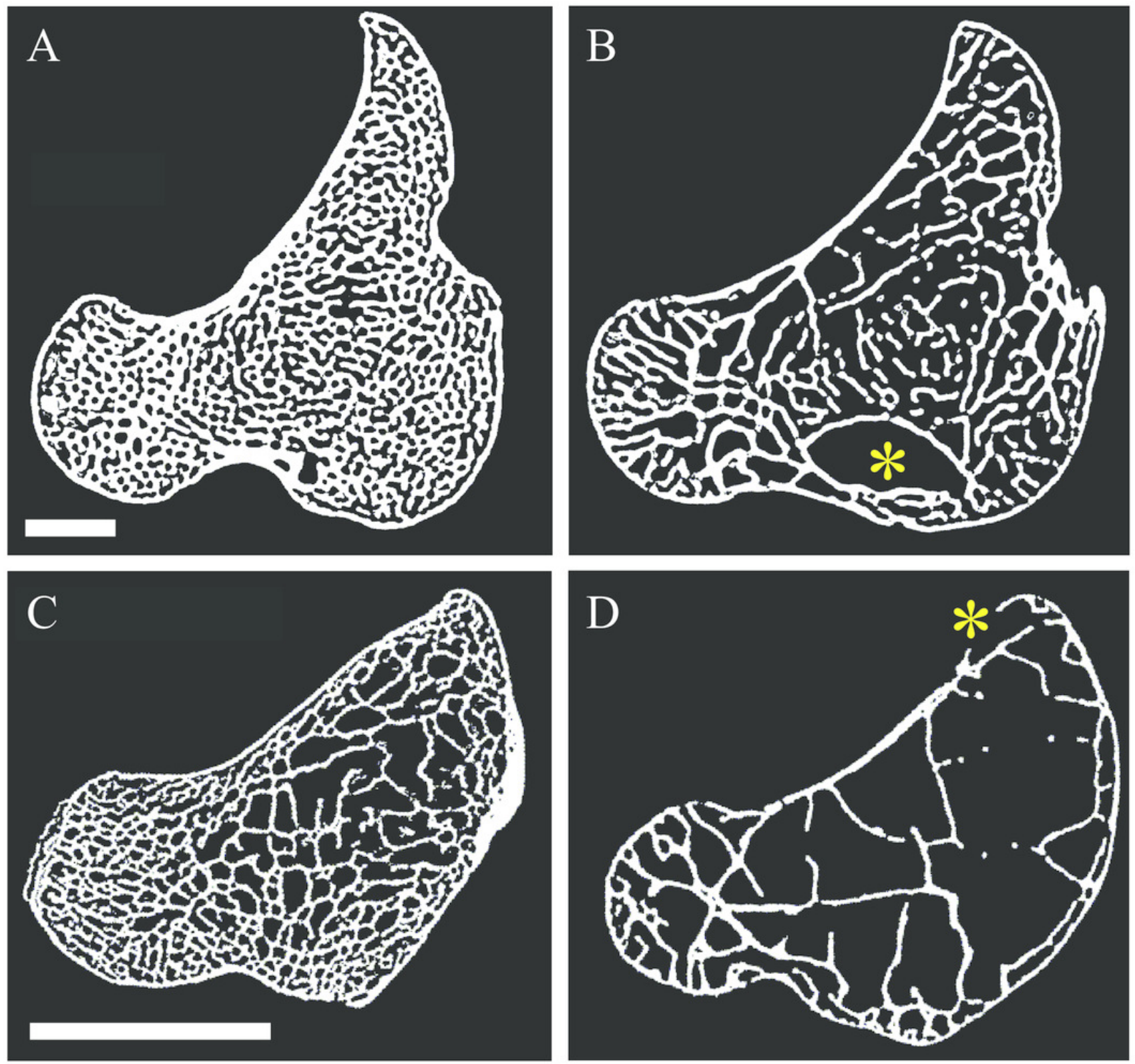


\section{Figure 15}

The main architectural features of cancellous bone in the human proximal femur.

(A) Vector field of $\mathbf{u}_{1}$ in the head, inferior neck and greater trochanter regions, plotted on a translucent rendering of the external bone geometry; view is in the coronal plane. Schematic inset illustrates close correspondence with the principal compressive (PC) and greater

trochanter (GT) groups of previous studies. (B) Vector field of $\mathbf{u}_{1}$ (red) and $\mathbf{u}_{2}$ (blue) in the middle of the metaphysis, viewed in the coronal plane. Schematic inset illustrates close correspondence with the secondary compressive (SC) and secondary tensile (ST) groups of previous studies. Note that both $\mathbf{u}_{1}$ and $\mathbf{u}_{2}$ are largely parallel to the coronal plane. (C) Vector field of $\mathbf{u}_{1}$ in the distal metaphysis and lesser trochanter (in oblique proximomedial view), which is largely parallel to the bone's long-axis. In this and all subsequent illustrations of fabric vector fields, the length of each fabric vector is proportional to its corresponding fabric eigenvalue. Additionally, all images are of bones from the right side of the body. 

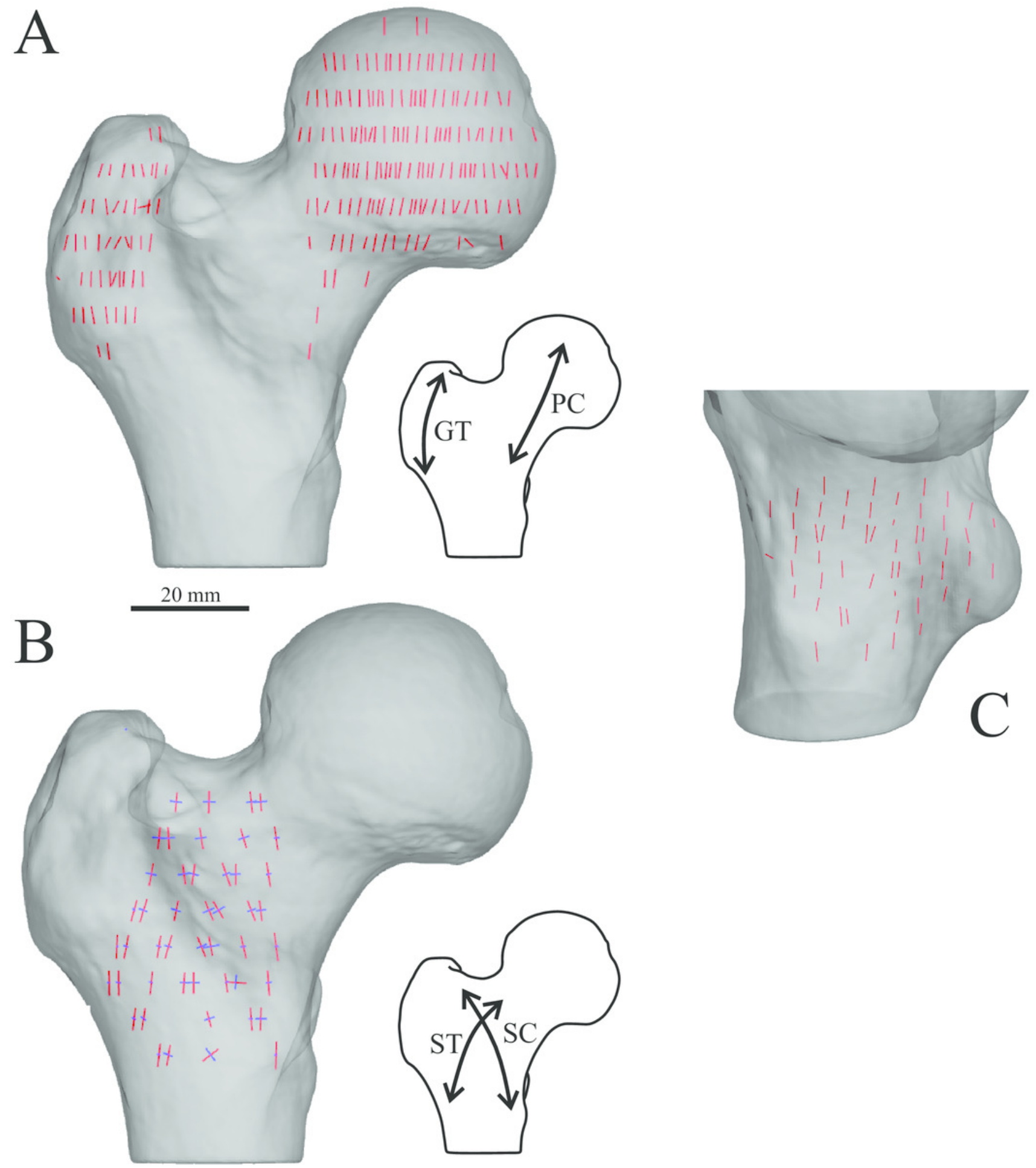


\section{Figure 16}

The main architectural features of cancellous bone in the proximal femur of birds.

(A-D) Vector field of $\mathbf{u}_{1}$ in the femoral head and inferior neck of an emu, Dromaius

novaehollandiae (QMO 16140; A, B), and ostrich, Struthio camelus (MV R.2385; C, D). (E-H)

Vector field of $\mathbf{u}_{1}$ under the facies antitrochanterica of a greater rhea, Rhea americana (QMO 23517; E, F), and chicken, Gallus gallus (PJB coll.; G, H). (I) Isosurface rendering of cancellous bone under the facies antitrochanterica of a dusky moorhen, Gallinula tenebrosa (PJB coll., between arrows), sectioned in the sagittal plane. (J-M) Vector field of $\mathbf{u}_{1}$ in the trochanteric crest of a southern cassowary, Casuarius casuarius (QMO 30105; J, K), and Struthio camelus (MV R.2385, L, M). (N) Isosurface rendering of cancellous bone in the trochanteric crest of a magpie goose, Anseranus semipalmata (QMO 29529, between arrows), sectioned in the sagittal plane. (O, P) Vector field of $\mathbf{u}_{1}$ throughout the entire proximal femur of Dromaius novaehollandiae (QMO 16140, O) and Casuarius casuarius (QMO 30105, P), which illustrates the increasing obliquity and disorganization of vectors in the distal metaphysis and transition to the diaphysis, shown in regions with braces. A, C, J and $L$ are anterior views; $B$ and $D$ are medial views; $E$ and $G$ are posterior views; $F, H, K$ and $M$ are lateral views; $O$ is an oblique anterolateral view; $P$ is an oblique anteromedial view. For reference, silhouettes of the animals depicted are provided in this figure and those that follow. 


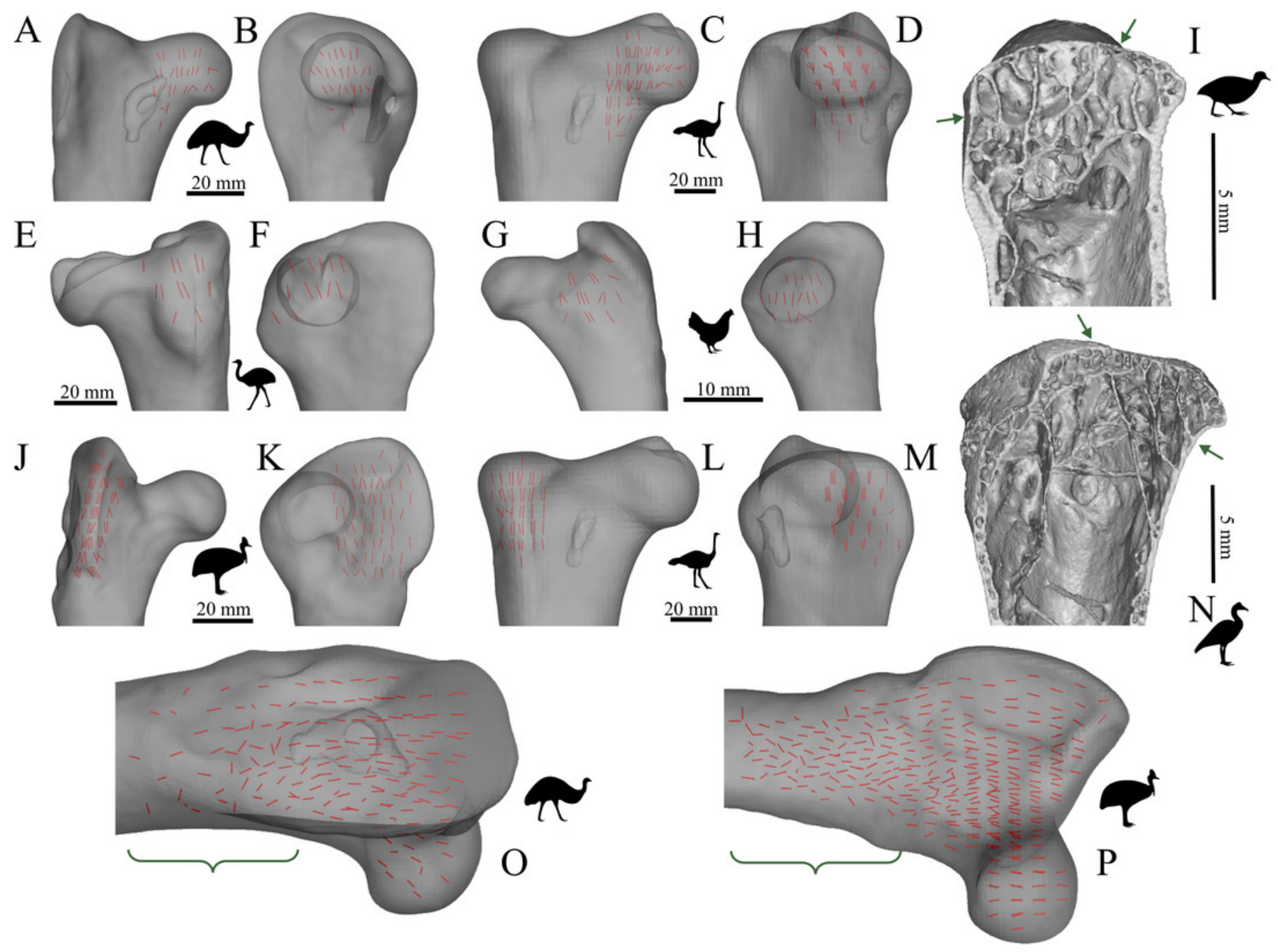




\section{Figure 17}

The main architectural features of cancellous bone in the proximal femur of extant sprawling reptiles.

(A, B) Vector field of $\mathbf{u}_{1}$ in the proximal femur of a freshwater crocodile, Crocodylus johnstoni (QMJ 47916). (C, D) Vector field of $\mathbf{u}_{1}$ in the proximal femur of a Spencer's goanna, Varanus spenceri (QMJ 84416). (E) Vector field of $\mathbf{u}_{1}$ throughout the proximal femur of a Komodo dragon, Varanus komodoensis (AM R.106933), which illustrates the increasing obliquity and disorganization of vectors in the distal metaphysis and transition to the diaphysis, shown in region with braces. A and C are anterior views ('dorsal view' of herpetologists); B and D are lateral views ('posterior view' of herpetologists); $E$ is an oblique anterolateral view. For clarity, the vectors of $\mathbf{u}_{1}$ in the fourth trochanter are not visible in $A$, $\mathrm{C}$ and $\mathrm{E}$. 


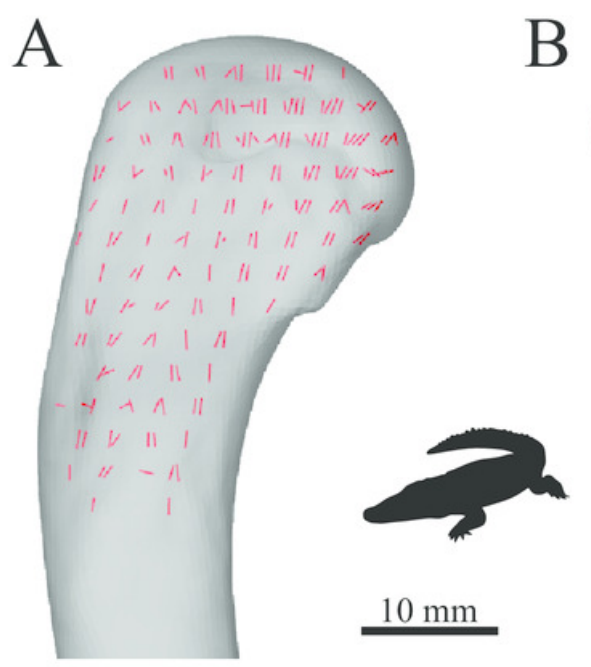

$\mathrm{C}$

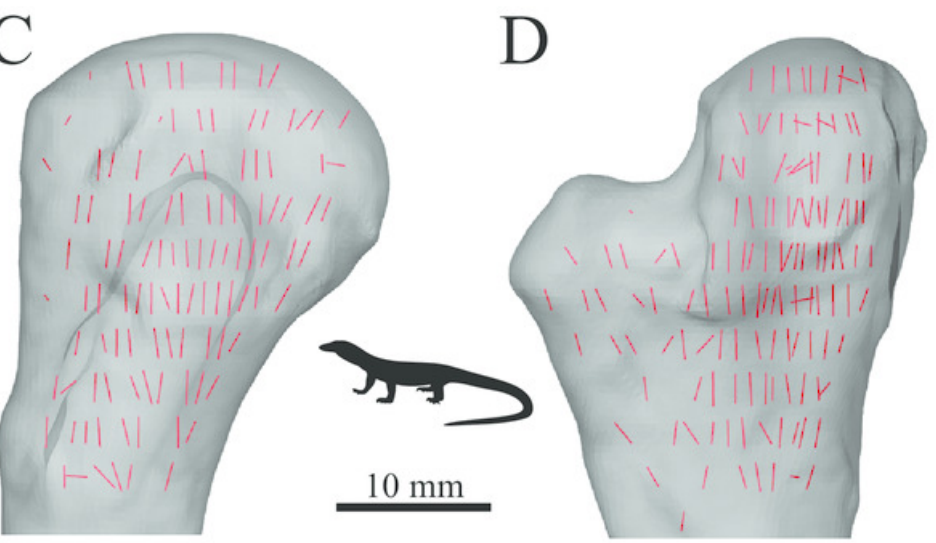

$\mathrm{E}$

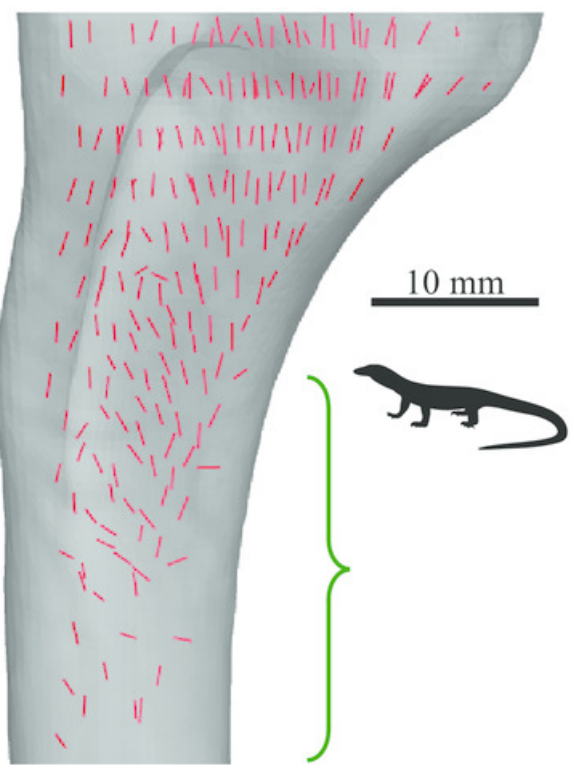


Figure 18

The orientation of $\mathbf{u}_{1}$ throughout the femoral head of Masiakasaurus knopfleri, here exemplified by FMNH PR 2117.

(A) Anterior view. (B) Lateral view. 


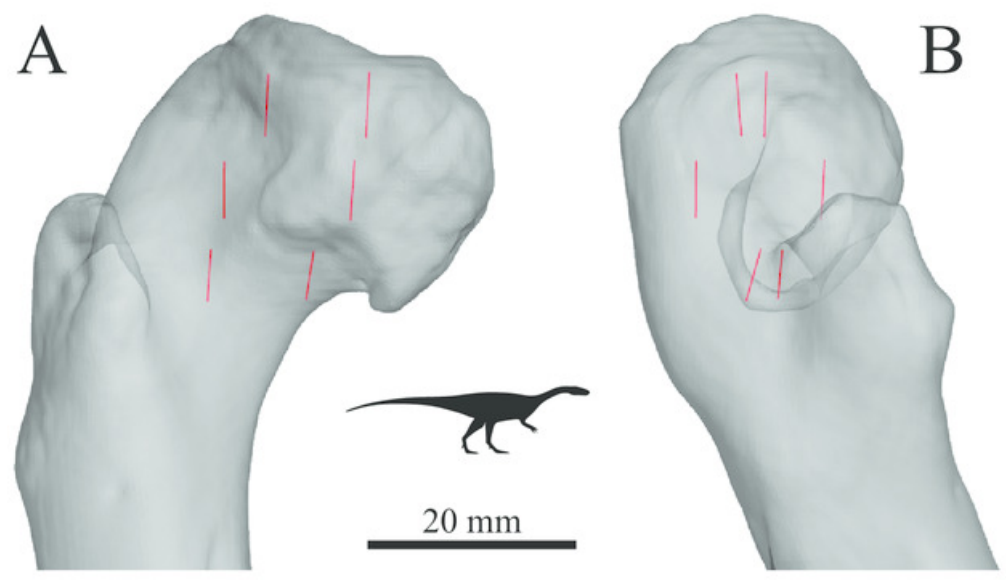




\section{Figure 19}

The main architectural features of cancellous bone in the proximal femur of both Allosaurus and the tyrannosaurids.

These are illustrated here with a 3-D geometric model of the observed architecture, mapped to the femur of Daspletosaurus torosus (TMP 2001.036.0001). (A-E) Five progressive rotations of the bone, in $30^{\circ}$ increments, from anteromedial to anterolateral views (C is a purely anterior view). (F) The observed orientation of the dominant tract of cancellous bone in the femoral head (blue) has a gentle anterior inclination; bone shown in medial view. For explanation of the features and colour coding, refer to the main text. Inset below $\mathrm{C}$ is a CT slice through the proximal femur of Tyrannosaurus rex (MOR 1128), parallel to the coronal plane and through the middle of the femoral head. This illustrates the very characteristic tract of cancellous bone that extends from the base of the femoral neck up towards the apex of the head, highly comparable to the tract present in humans (cf. Fig. 4A).

A

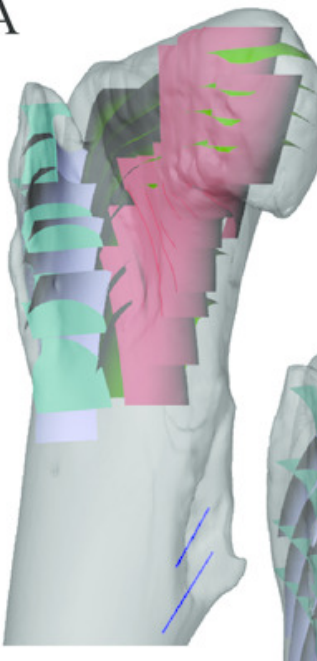

$\mathrm{C}$
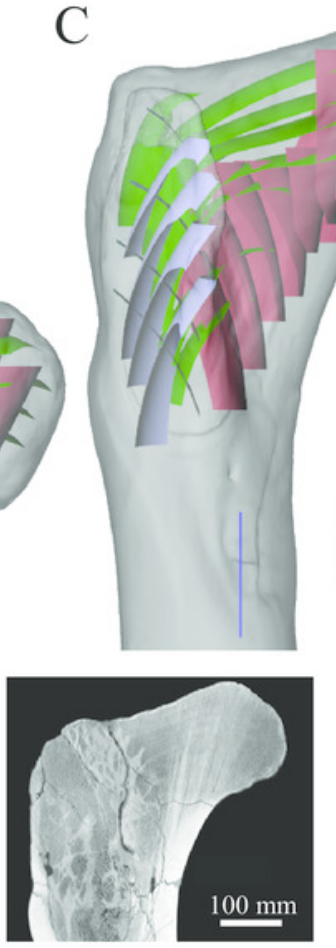

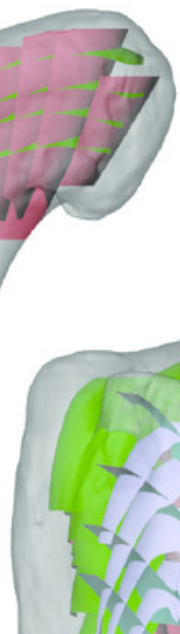

E

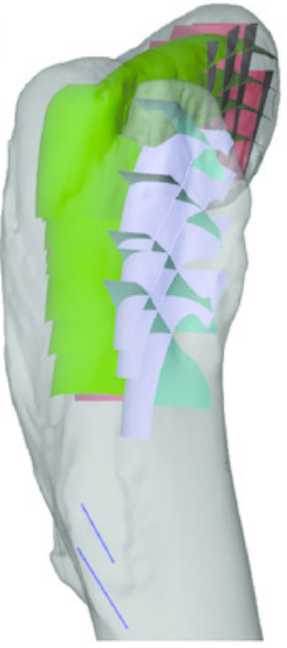

$\mathrm{B}$

$100 \mathrm{~mm}$

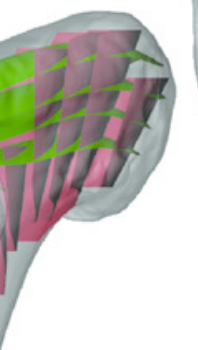

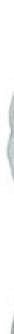

$\mathrm{D}$

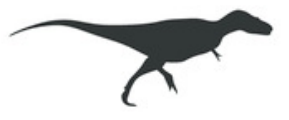

F 


\section{Figure 20}

The main architectural features of cancellous bone in the proximal femur of ornithomimids and caenagnathids.

(A-D) Vector field of $\mathbf{u}_{1}(\mathrm{~A}, \mathrm{C})$ and $\mathbf{u}_{2}(\mathrm{~B}, \mathrm{D})$ in the femoral head and proximal metaphysis of an inteterminate ornithomimid (TMP 85.36.276), in oblique anteromedial (A, B) and oblique anterolateral (C, D) views. Note that the vector field along the anterior and posterior peripheries of the femoral head are not shown here (for clarity), where they are more typically oriented as in birds and humans. (E) Vector field of $\mathbf{u}_{1}$ in the greater trochanter region and distal metaphysis of an indeterminate ornithomimid (TMP 85.36.276), in oblique anterolateral view; note the increased obliquity and disorganization of vectors in the distal metaphysis (region with braces). (F) Vector field of $\mathbf{u}_{1}$ in the lesser trochanter of an indeterminate ornithomimid (TMP 91.36.569), in oblique anteromedial view. (G) Vector field of $\mathbf{u}_{1}$ in the proximal femur of an indeterminate caenagnathid (TMP 86.36.323), in a 3-D slice parallel to the axial plane and through the femoral head and lesser trochanter. Main image is shown in axial view (anterior is toward bottom of page), with inset illustrating the region illustrated in context of the whole bone. The medialmost part of the femoral head is missing (dotted line). 


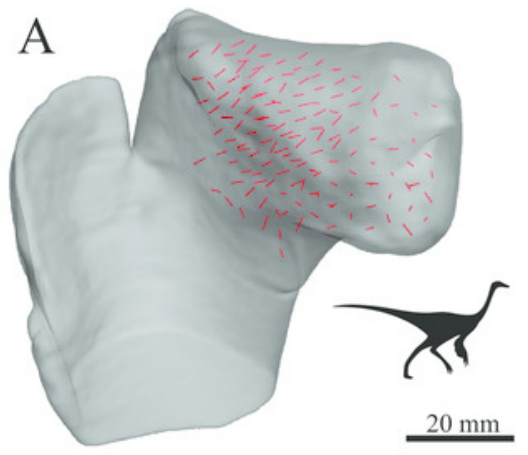

B
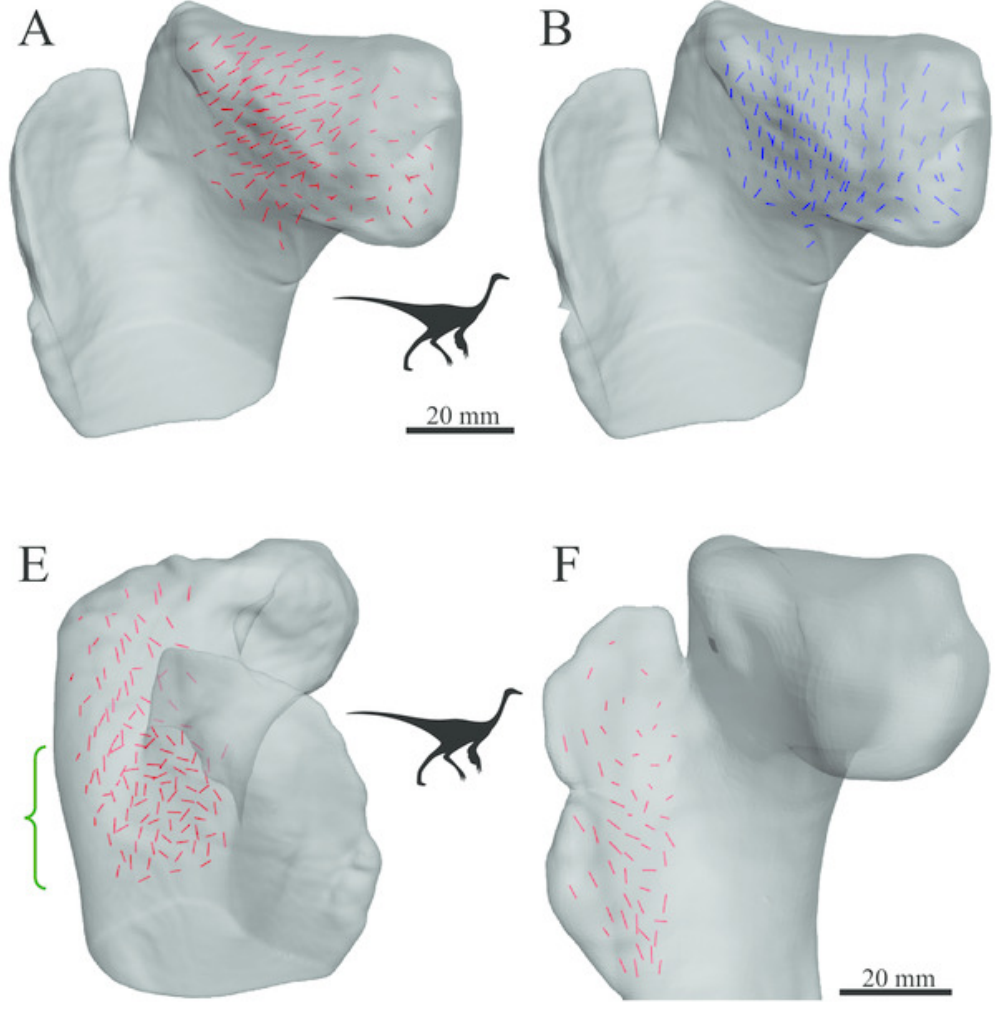

F

$20 \mathrm{~mm}$
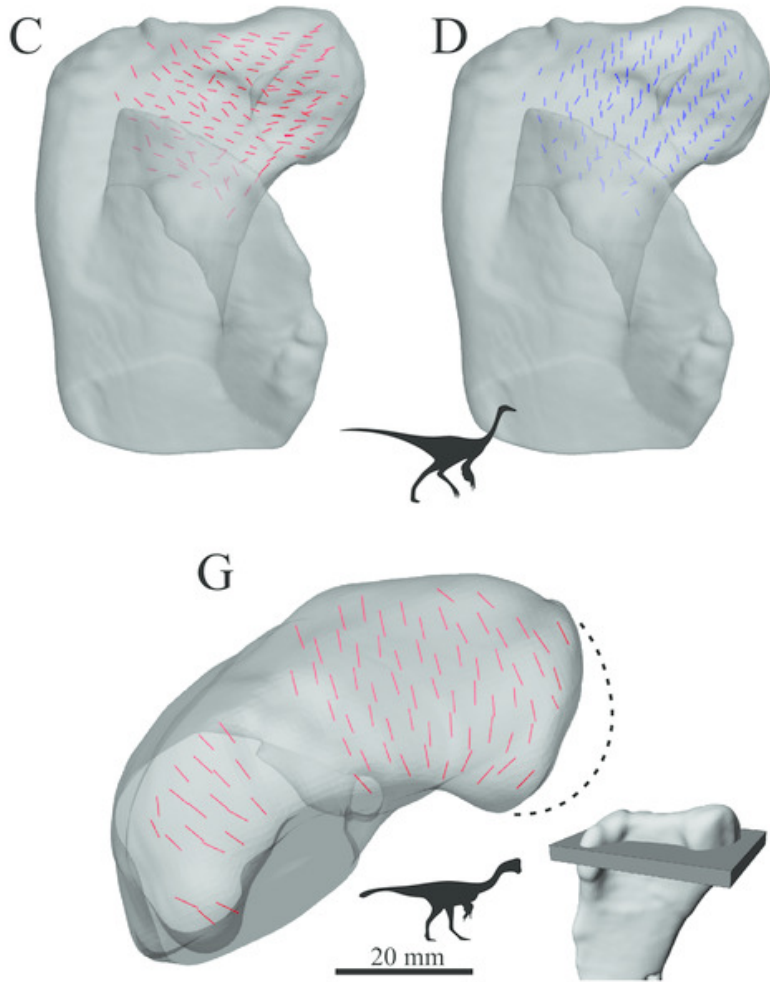


\section{Figure 21}

The main architectural features of cancellous bone in the proximal femur of Falcarius utahensis and Troodontidae sp.

(A) Vector field of $\mathbf{u}_{1}$ in the proximal femur of Falcarius (UMNH VP 12361), viewed as a 3-D slice parallel to the coronal plane and through the middle of the bone. Schematic inset illustrates three main trajectories in this image, which are not too dissimilar from the patterns observed in humans and large non-avian theropods (cf. Figs 15, 19). (B, C) Vector field of $\mathbf{u}_{1}$ (B) and $\mathbf{u}_{2}$ (C) in the lesser trochanter of Falcarius, in oblique anterolateral view. (D) Vector field of $\mathbf{u}_{2}$ in the proximal femur of Falcarius, in a 3-D slice parallel to the axial plane and through the femoral head. Main image is shown in axial view (anterior is toward bottom of page), with inset illustrating the region illustrated in context of the whole bone. $(E, F)$ Vector field of $\mathbf{u}_{1}$ in the femoral head and inferior neck of Troodontidae sp. (MOR 748) in anterior (E) and medial ( $\mathbf{F}$ ) views. (G, H) Vector field of $\mathbf{u}_{1}$ in the region of the greater trochanter of Troodontidae sp. (MOR 553s-7.28.91.239) in posterior (G) and lateral $(\mathrm{H})$ views. (I) Orientation of $\mathbf{u}_{1}$ in the lesser trochanter, or immediate region thereof, of Troodontidae sp. , in oblique anterolateral view (main image illustrates MOR 748; inset illustrates MOR 553s-7.28.91.239). (J) Vector field of $\mathbf{u}_{1}$ throughout the metaphysis of Troodontidae sp. (MOR 553s-7.28.91.239), illustrating increasing obliquity and disorganization of vectors in the distal metaphysis and transition to the diaphysis (region with braces). 

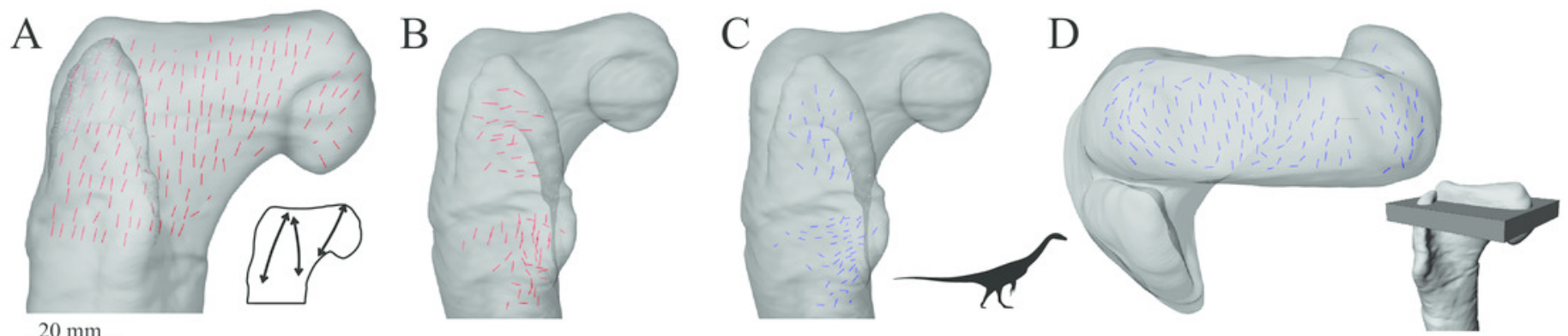

$\underline{20 \mathrm{~mm}}$
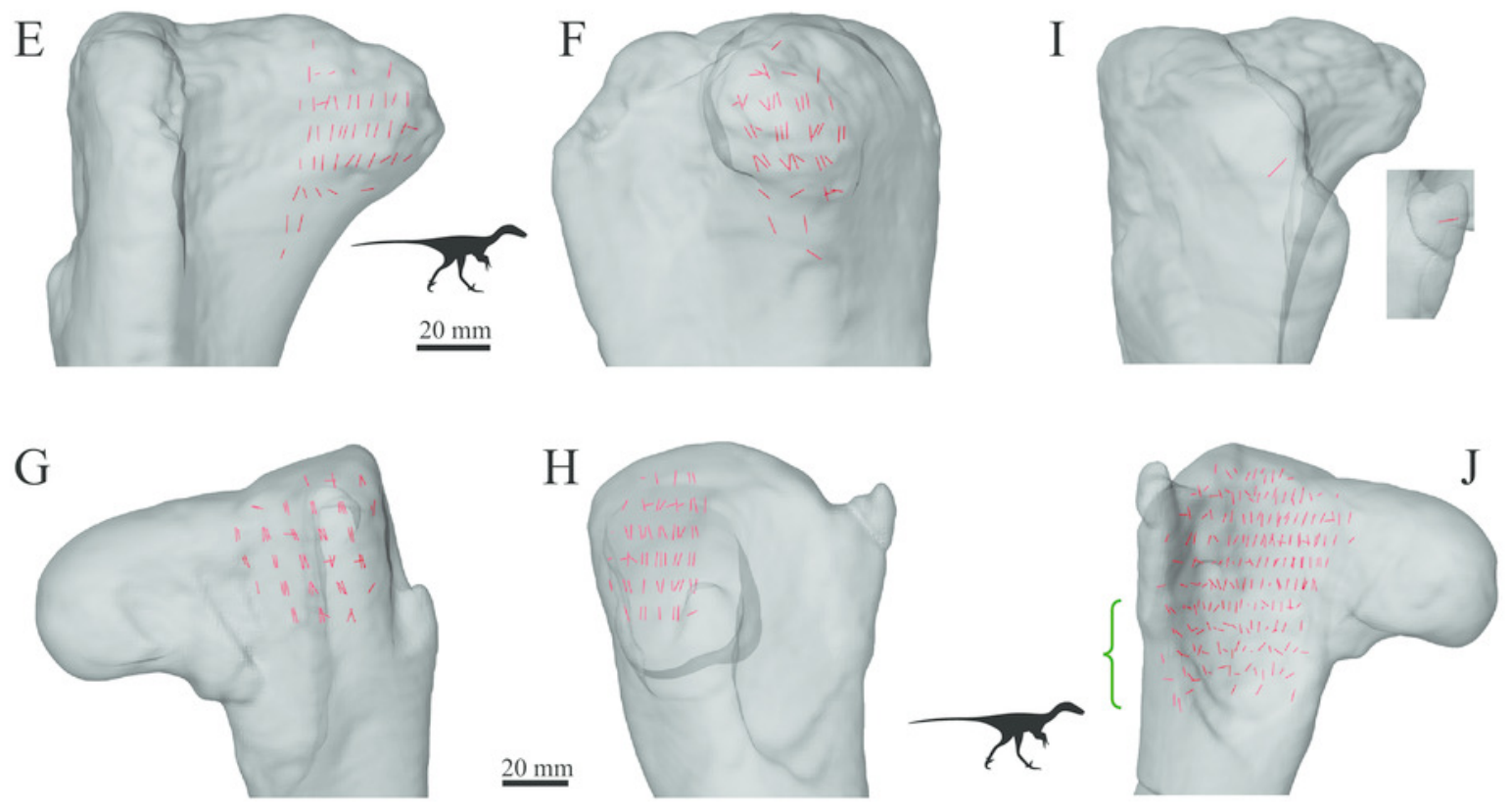


\section{Figure 22}

The mean orientation of $\mathbf{u}_{1}$ in the femoral head, referenced in the femur anatomical coordinate system.

This is plotted on an equal-angle stereoplot, with northern hemisphere projection (using StereoNet 9.5Allmendinger et al. 2013; Cardozo \& Allmendinger 2013) . (A) Results for all specimens analysed. Note that for the fossil specimens, only those that were complete and well-preserved, and enabled an anatomical coordinate system to be defined, were analysed. Colour codes: black $=$ birds, pink $=$ human, blue $=$ Masiakasaurus $(F M N H$ PR 2153, UA 8384) , orange $=$ Falcarius, green $=$ Troodontidae sp. $($ MOR 748), purple $=$ general orientation for Allosaurus and the tyrannosaurids, red = mean orientation across birds. (B) Results for species or genera that were multiply sampled often displayed significant instraspecific or intrageneric variation, ranging up to $30.6^{\circ}$. Colour codes: black $=$ ostrich, Struthio camelus, blue $=$ southern cassowary, Casuarius casuarius , orange $=\mathrm{emu}$, Dromaius novaehollandiae, red = chicken, Gallus gallus , green = kiwi, Apteryx spp. (C, D) The orientation of $\mathbf{u}_{1}$ superimposed on the $\mathrm{CT}$ scan of a representative tyrannosaur femur ( Daspletosaurus torosus , TMP 2001.036.0001) in coronal (C) and sagittal (D) views, illustrating how the orientation data extracted and illustrated in the geometric models (e.g., Fig. 19) qualitatively reflects the observed cancellous bone architecture in the scans. 


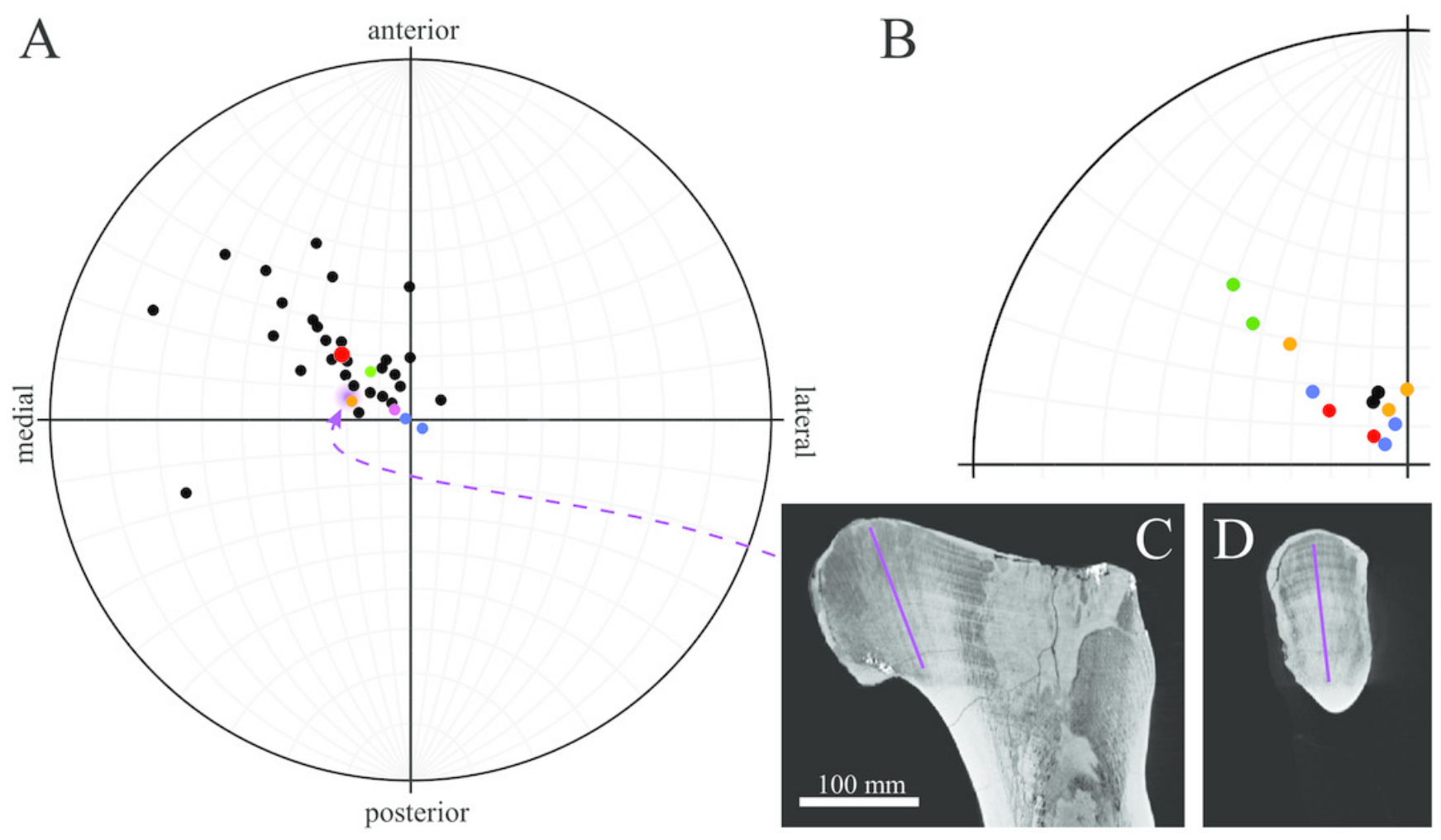




\section{Figure 23}

The main architectural features of cancellous bone in the human distal femur.

(A) Vector field of $\mathbf{u}_{1}$ in a 3-D slice parallel to the coronal plane, made through the middle of the condyles. Schematic inset illustrates weakly developed double-arcuate pattern. (B) Vector field of $\mathbf{u}_{1}$ in a 3-D slice parallel to the sagittal plane, made between the condyles. Schematic inset illustrates weakly developed double-arcuate pattern. (C) Vector field of $\mathbf{u}_{1}$ in the medial condyle, shown for a 3-D slice parallel to the sagittal plane, made through the middle of the condyle. (D) Vector field of $\mathbf{u}_{2}$ at the level of the condyles, shown for a 3-D slice parallel to the axial plane, made through the middle of the condyles; anterior is toward top of page. Schematic inset illustrates the two distinctive tracts that comprise a 'butterfly pattern'. 
A

A
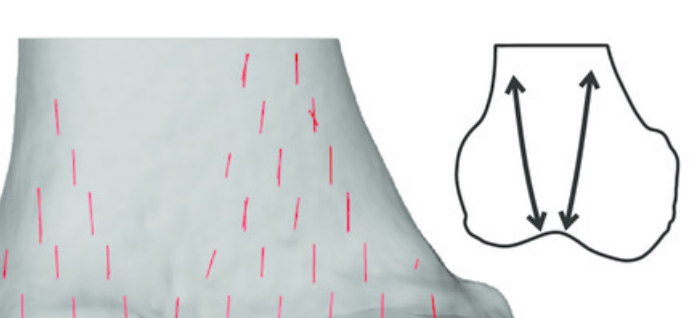

B

$\left(\begin{array}{lllllllll}1 & 1 & 7 & 1 & -1 & 1 & 1 & 1 & 1 \\ 1 & 1 & 1 & 1 & 1 & 1 & 1 & 1 & 1\end{array}\right)$
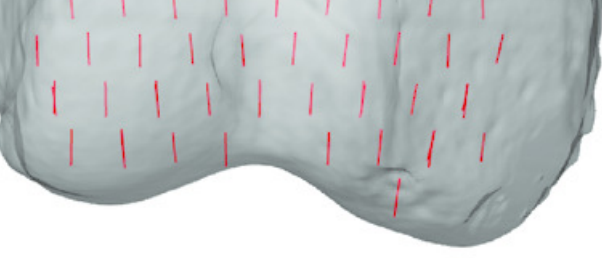

$20 \mathrm{~mm}$

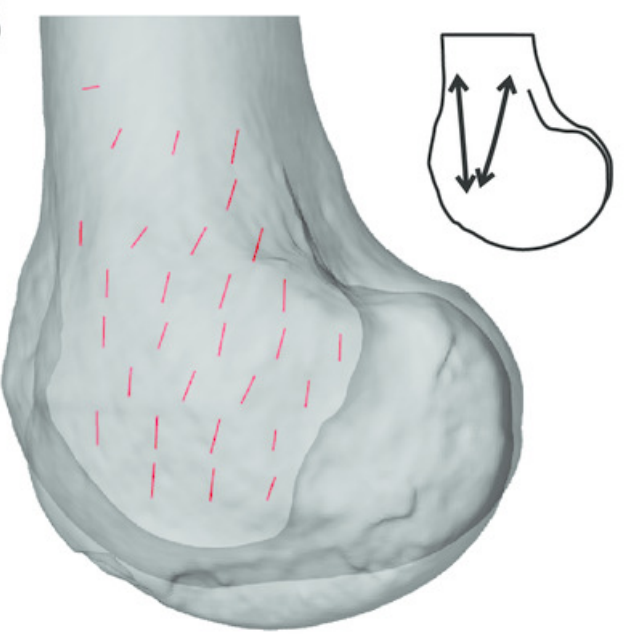

$\mathrm{C}$

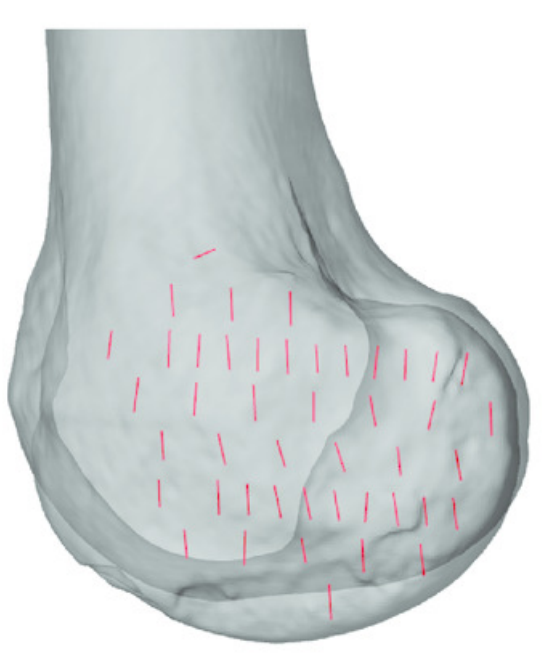

$\mathrm{D}$

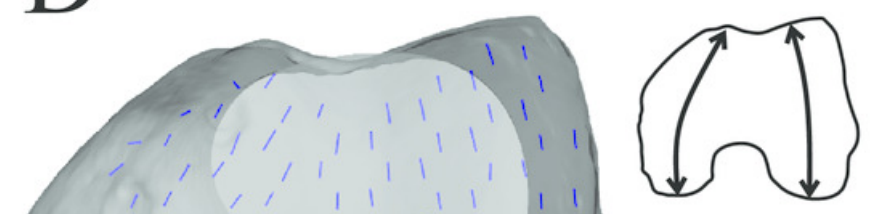




\section{Figure 24}

The main architectural features of cancellous bone in the distal femur of birds.

(A) Vector field of $\mathbf{u}_{1}$ in the central metaphysis of a southern cassowary, Casuarius casuarius (QMO 30105), in a 3-D slice, parallel to the sagittal plane and between the condyles, shown in lateral view. Note the weakly developed double arcuate pattern. (B, C) Vector field of $\mathbf{u}_{2}$ in a 3-D slice through the middle of the condyles in an ostrich, Struthio camelus (MV R.2385, B), and a emu, Dromaius novaehollandiae (QMO 11685, C), shown in distal view. Note the 'butterfly pattern' in both examples. (D) Isosurface rendering of cancellous bone in the distal condyles of a Japanese quail, Coturnix japonica (PJB coll.), sectioned in the axial plane; notice the 'butterfly pattern' between the arrows. (E-H) Vector field of $\mathbf{u}_{1}$ in the medial condyle of Dromaius novaehollandiae (QMO 16140, E, F) and Casuarius casuarius (QMO 30604, G, H), shown in anterior $(E, G)$ and medial $(F, H)$ views. (I, J) Vector field of $\mathbf{u}_{1}$ in the lateral condyle of a chicken, Gallus gallus (PJB coll.), shown in anterior (I) and lateral (J) views. (K) Isosurface rendering of cancellous bone in the medial condyle of a purple swamphen, Porphyrio porphyrio (PJB coll.), sectioned in the sagittal plane. (L) Vector field of $\mathbf{u}_{1}$ throughout the entire distal femur of Casuarius casuarius (QMO 31137), illustrating increasing obliquity and disorganization of vectors in the proximal metaphysis and transition to the diaphysis (region with braces). 

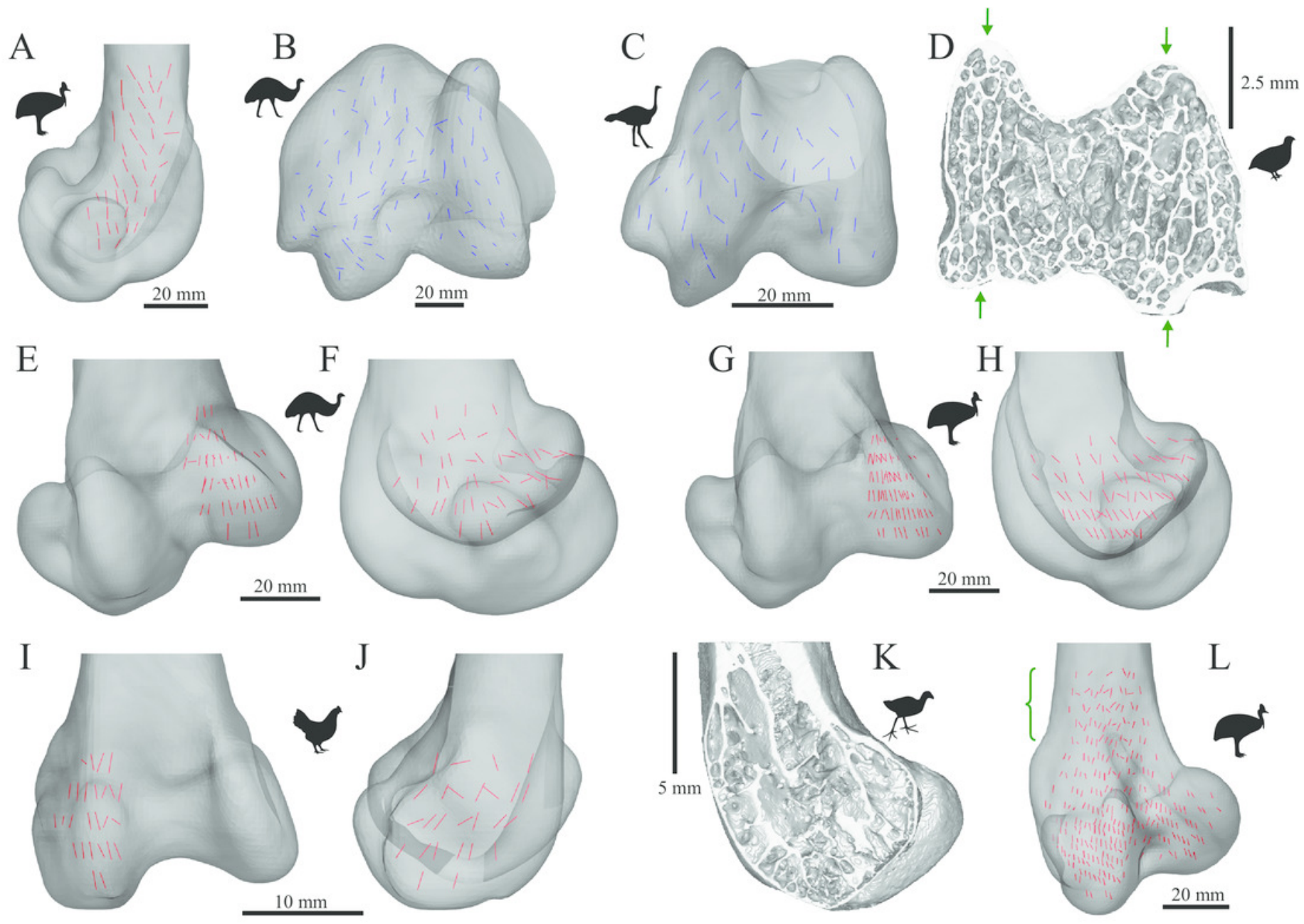


\section{Figure 25}

The main architectural features of cancellous bone in the distal femur of extant sprawling reptiles.

(A, B) Vector field of $\mathbf{u}_{2}$ in a 3-D slice through the middle of the condyles in a freshwater crocodile, Crocodylus porosus (QMJ 48127, A), and a Komodo dragon, Varanus komodoensis (AM R.106933, B), shown in proximal view. (C, D) Vector field of $\mathbf{u}_{1}$ in the medial condyle of Crocodylus porosus (QMJ 48127), shown in anterior (C) and medial (D) views. (E, F) Vector field of $\mathbf{u}_{1}$ in the lateral condyle of a Spencer's goanna, Varanus spenceri (QMJ 484416), shown in anterior $(E)$ and lateral $(F)$ views. 

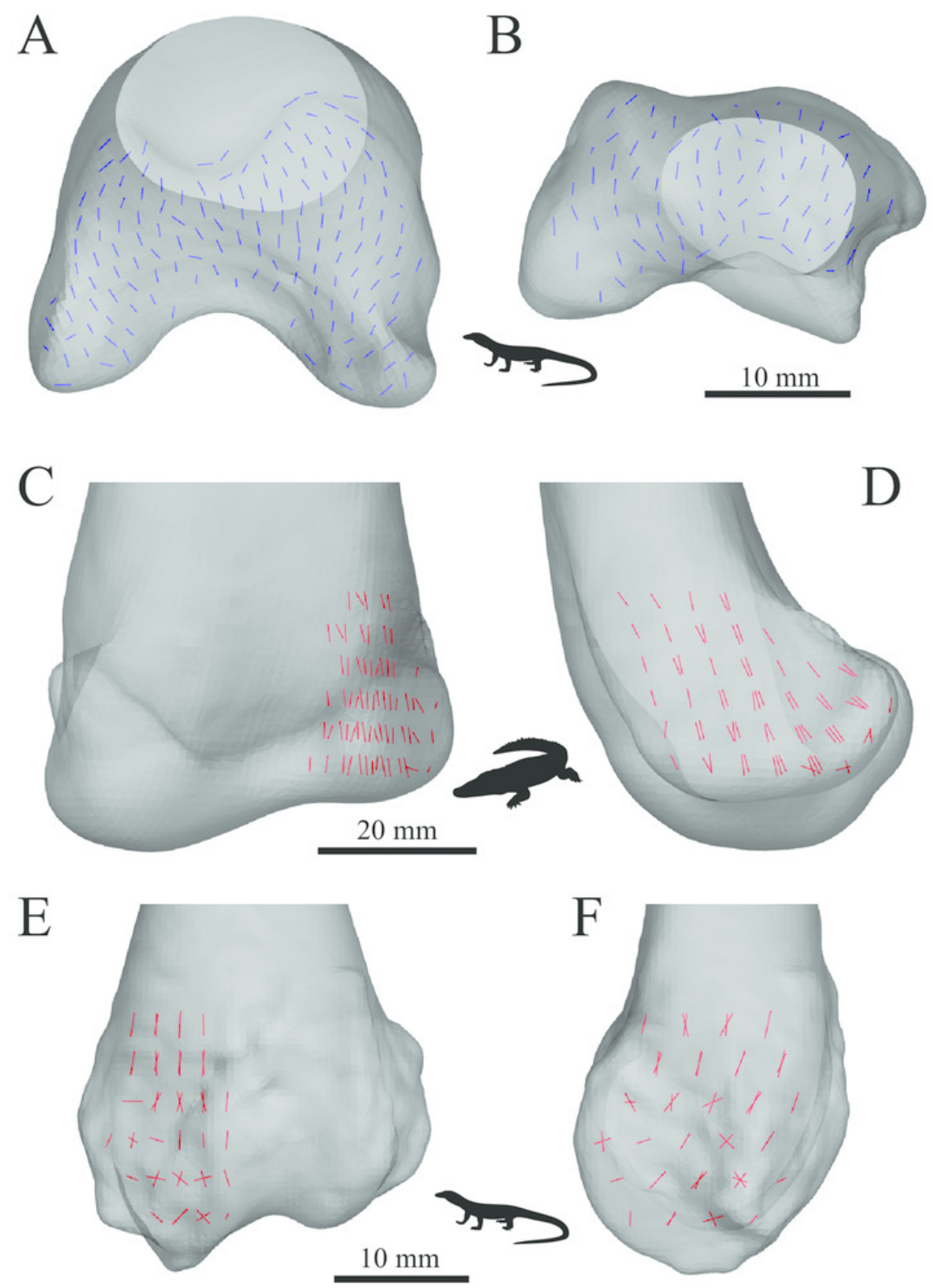


\section{Figure 26}

The main architectural features of cancellous bone in the distal femur of both Allosaurus and the tyrannosaurids.

These are illustrated here with a 3-D geometric model of the observed architecture, mapped to the femur of Daspletosaurus torosus (TMP 2001.036.0001). (A-G) Seven progressive rotations of the bone, in $30^{\circ}$ increments, from medial to lateral views ( $D$ is a purely anterior view). Note that the architecture of the metaphysis was not observed in the Allosaurus specimens studied, owing to insufficient contrast or resolution in the CT scans failing to reveal any information about the metaphysis. For explanation of the features and colour coding, refer to the main text.

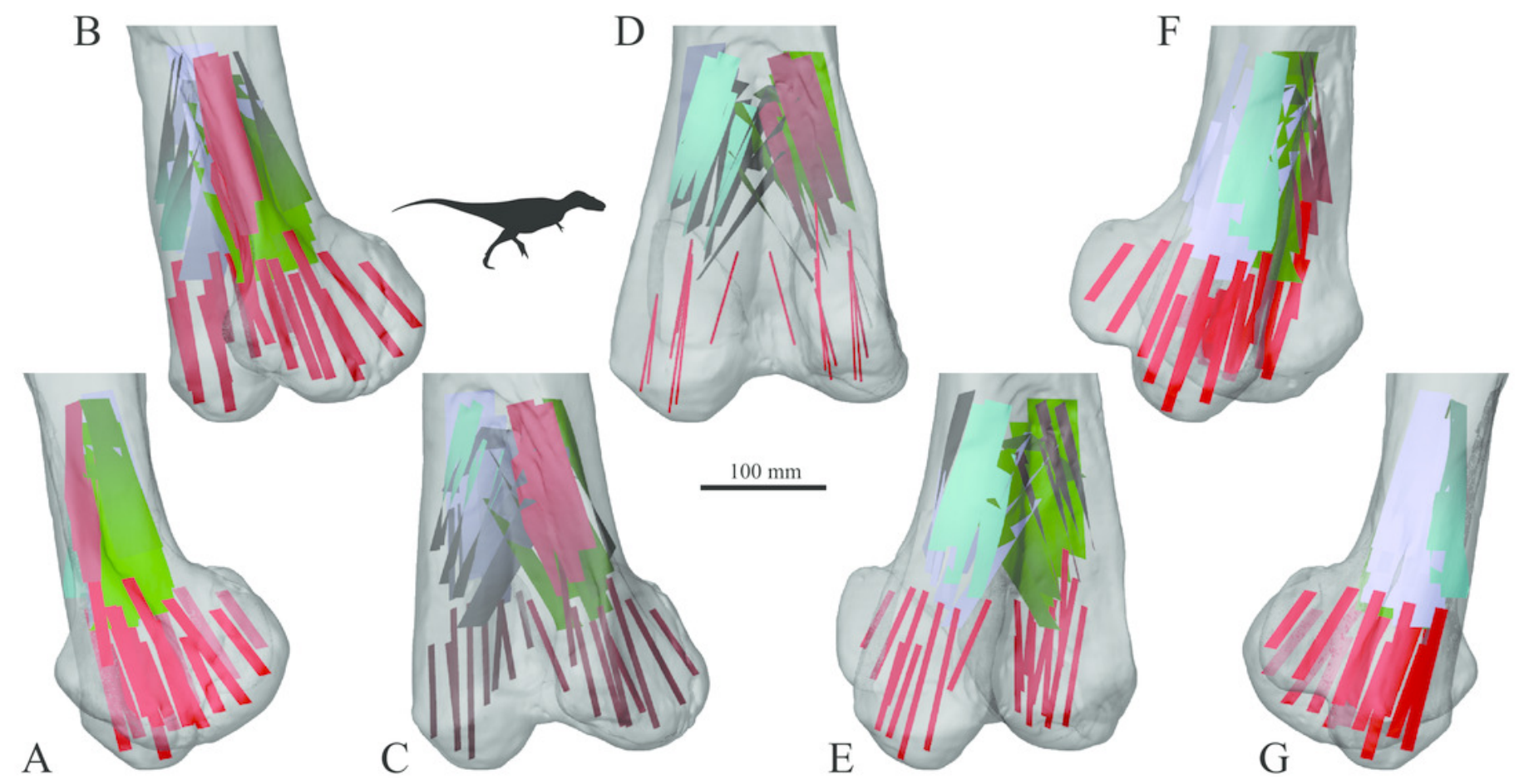




\section{Figure 27}

The main architectural features of cancellous bone in the distal femur of ornithomimids and caenagnathids.

(A, B) Vector field of $\mathbf{u}_{1}$ in the lateral condyle of an indeterminate ornithomimid (TMP 99.55.337) in posterior (A) and lateral (B) views. (C, D) Vector field of $\mathbf{u}_{1}$ in the medial condyle of an indeterminate caenagnathid (TMP 86.36.323) in posterior (C) and medial (D) views. $(E, F)$ Vector field of $\mathbf{u}_{1}(E)$ and $\mathbf{u}_{2}(F)$ in the distal femur of an indeterminate ornithomimid (TMP 91.36.569) at the level of the distal condyles, shown in proximal view for a 3-D slice parallel to the axial plane (inset shows location of slice). In A-D, the highlighted yellow vectors in the posterior extremities of the condyles have a much more mediolateral orientation compared to elsewhere in the condyle. This is also seen in $\mathrm{E}$, where vectors that appear longer are more parallel to the axial plane, and vectors that appear shorter are more proximodistally oriented. 

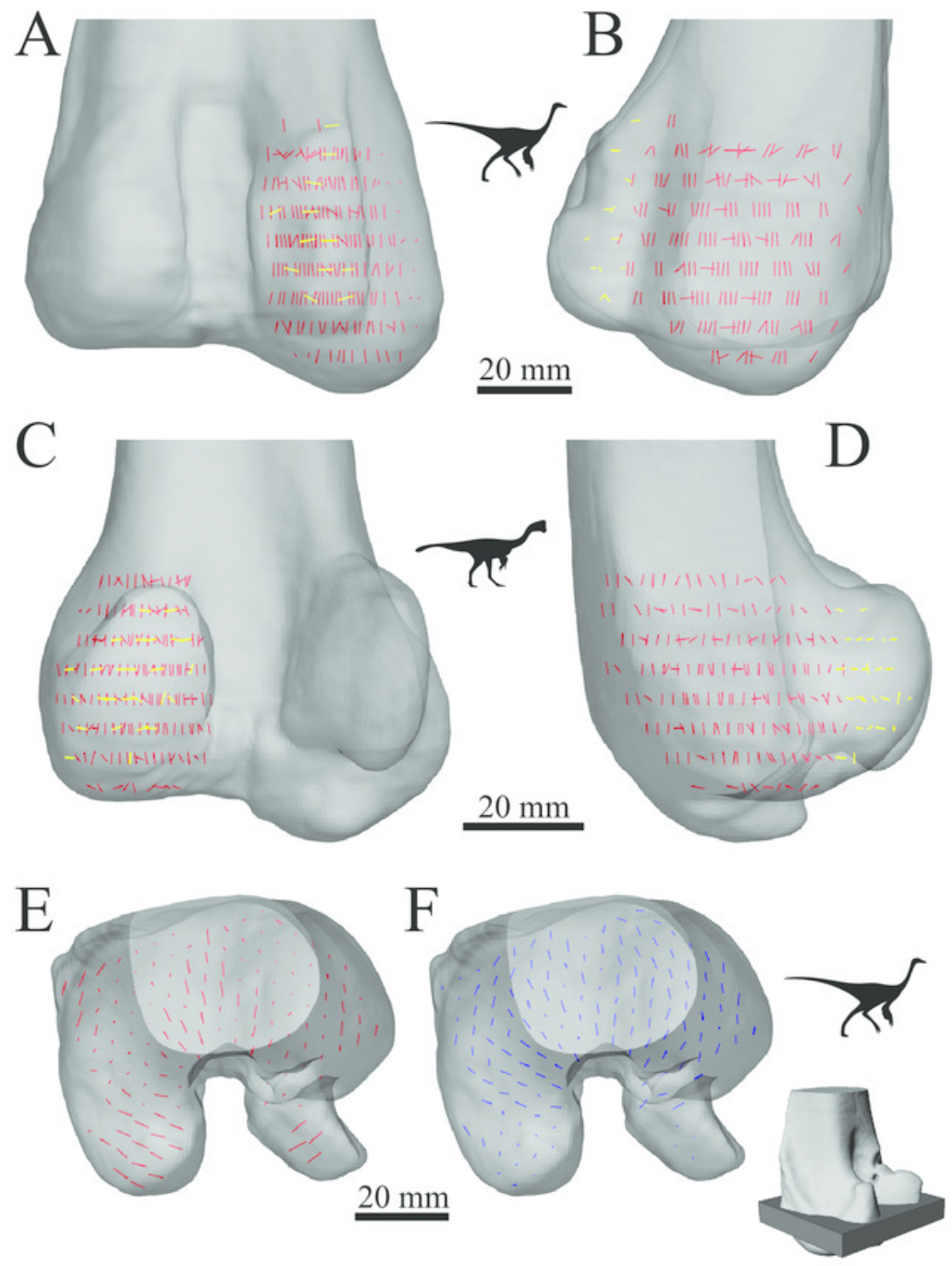


\section{Figure 28}

The main architectural features of cancellous bone in the distal femur of Falcarius utahensis and Troodontidae sp.

(A, B) Vector field of $\mathbf{u}_{1}$ in the medial condyle of Falcarius (UMNH VP 12360) in anterior (A) and medial (B) views. (C) Vector field of $\mathbf{u}_{1}$ throughout the distal femur of Troodontidae sp. (MOR 553s-7.28.91.239), illustrating increasing obliquity and disorganization of vectors in the proximal metaphysis and transition to the diaphysis (region with braces). (D, E) Vector field of $\mathbf{u}_{1}$ in the lateral condyle of Troodontidae sp. (MOR 748) in anterior (D) and lateral (E) views. (F) Vector field of $\mathbf{u}_{2}$ in the condyles of Troodontidae sp. (MOR 748), shown as a 3-D slice through the middle of the condyles in axial view; anterior is toward top of page. 


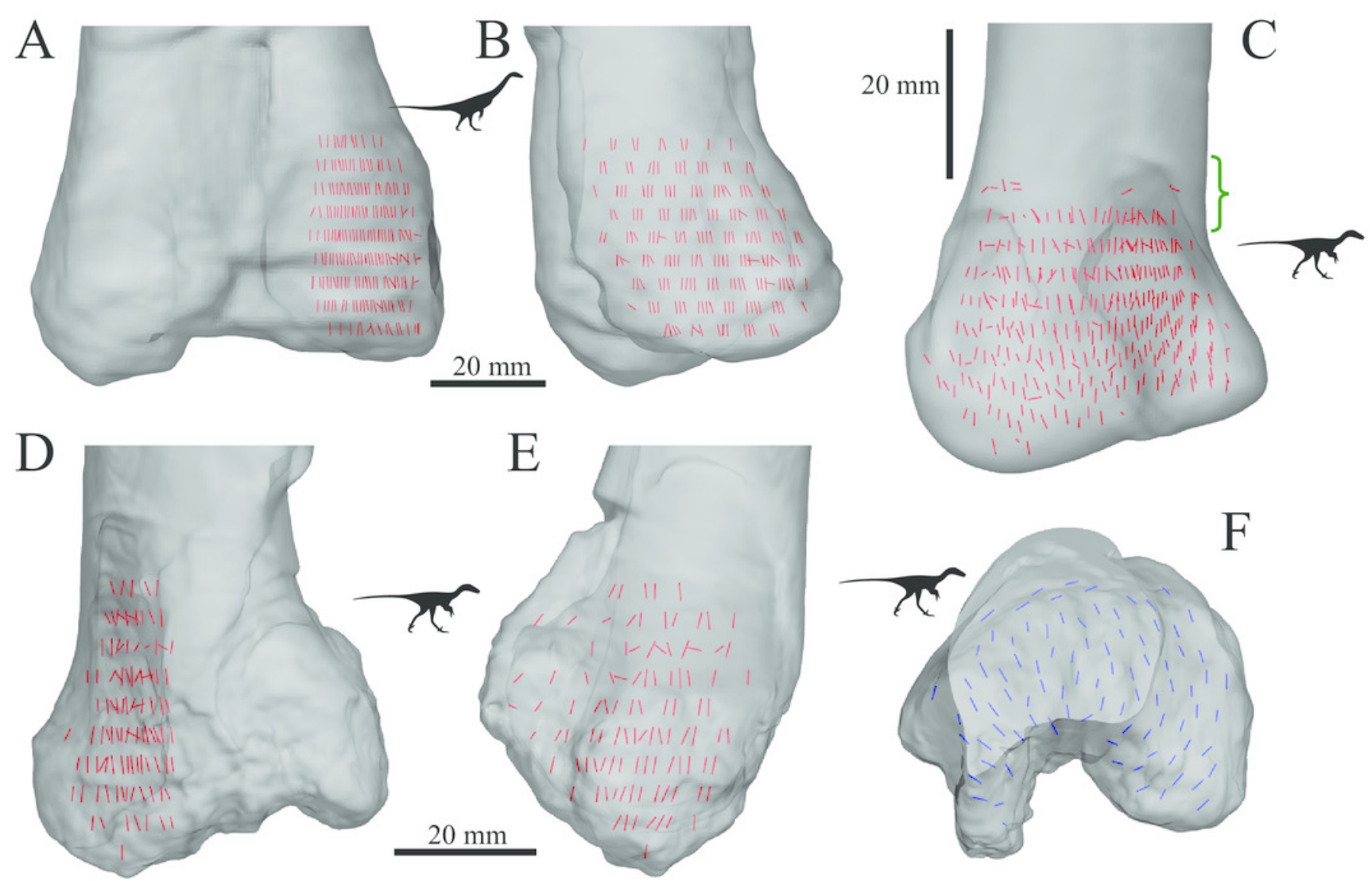




\section{Figure 29 (on next page)}

The mean orientation of $\mathbf{u}_{1}$ in the medial femoral condyle, referenced in the femur anatomical coordinate system.

This is plotted on an equal-angle stereoplot, with southern hemisphere projection (using StereoNet 9.5). (A) The results for all specimens analysed; for clarity, only the posteromedial quadrant of the plot is shown. Note that for the fossil specimens, only those that were complete and well-preserved, and enabled an anatomical coordinate system to be defined, were analysed. Colour codes: black $=$ birds, pink $=$ human, blue $=$ Masiakasaurus (FMNH PR 2153, UA 8384), orange $=$ Falcarius, green $=$ Troodontidae sp. , purple $=$ general orientation for Allosaurus and the tyrannosaurids, yellow $=$ indeterminate ornithomimid (TMP 91.36.569), brown $=$ indeterminate caenagnathid, red $=$ mean orientation across birds. (B) Comparison of posterior inclination of $\mathbf{u}_{1}$ in sagittal plane versus femur length in birds, with major axis regression line (and associated statistics) plotted. 


\section{Figure 30}

The main architectural feature of cancellous bone in the human proximal tibia is the gentle posterior inclination of $\mathbf{u}_{1}$ underneath the medial and lateral condyles.

(A) Vector field of $\mathbf{u}_{1}$ under the lateral condyle, in lateral view. (B) Vector field of $\mathbf{u}_{1}$ under both condyles, in anterior view. (C) Vector field of $\mathbf{u}_{1}$ under the medial condyle, in medial view. 

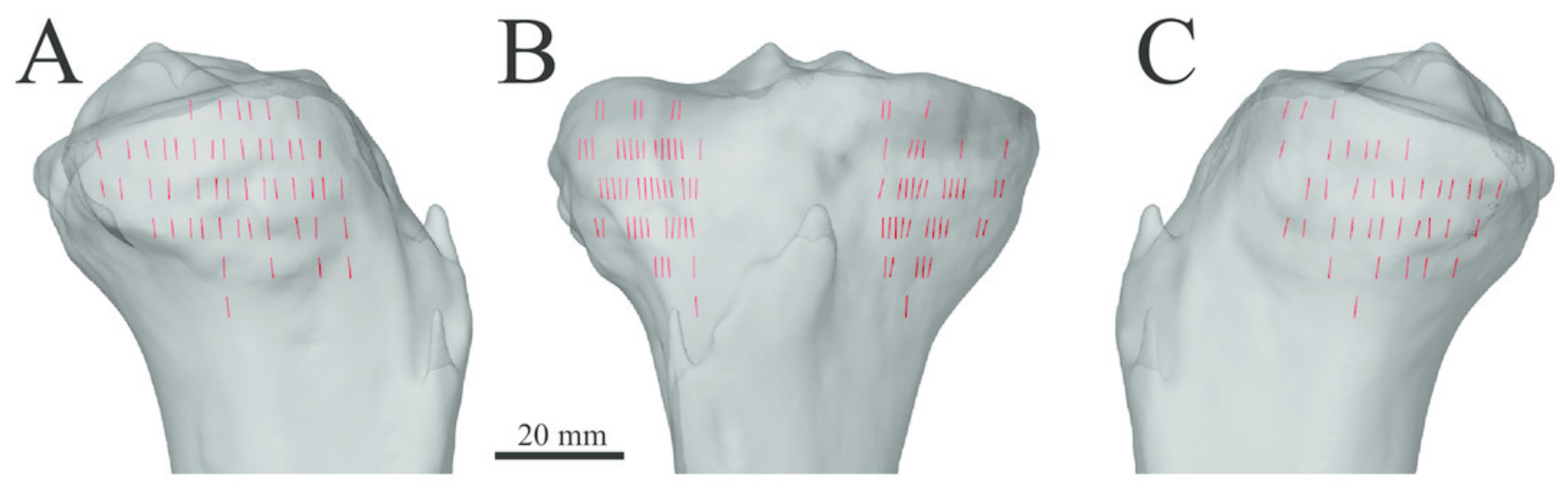


\section{Figure 31}

The main architectural features of cancellous bone in the proximal tibia of birds.

(A, B) Vector field of $\mathbf{u}_{1}$ in the anterior (cranial) cnemial crest of an emu, Dromaius novaehollandiae (QMO 11686, A), and turkey, Meleagris gallopavo (RVC turkey 1, B), shown in medial view. (C, D) Vector field of $\mathbf{u}_{1}$ in the lateral cnemial crest of a southern cassowary, Casuarius casuarius (QMO 30105), shown in anterior (C) and lateral (D) views. (E) Isosurface rendering of cancellous bone in the anterior cnemial crest of an Australian white ibis, Threskiornis moluccus (PJB coll., between arrows), sectioned in the sagittal plane. (F) Isosurface rendering of cancellous bone in the lateral cnemial crest of a guineafowl, Numida meleagris (PJB coll., between arrows), sectioned in the plane of the crest. (G-J) Vector field of $\mathbf{u}_{1}$ under the medial condyle of an ostrich, Struthio camelus (MV R.2385, G, H), and chicken, Gallus gallus (PJB coll., I, J), shown in posterior ( $G, I)$ and medial $(H, J)$ views. (K) Isosurface rendering of cancellous bone under the medial condyle of an elegant-crested tinamou, Eudromia elegans (UMZC 404.e, between arrows), sectioned in the sagittal plane. (L-O) Vector field of $\mathbf{u}_{1}$ under the lateral condyle of Struthio camelus (MV R.2711, L, M) and Dromaius novaehollandiae (QMO 11686, N, O), shown in posterior (L, N) and lateral (M, 0 ) views. (P) Isosurface rendering of cancellous bone under the lateral condyle of a little spotted kiwi, Apteryx owenii (UMZC 378.iii, between arrows), sectioned in the coronal plane. (Q) Vector field of $\mathbf{u}_{1}$ in a 3-D slice through the middle of the proximal metaphysis, cnemial crests and condyles of Dromaius novaehollandiae (QMO 11686), parallel to the sagittal plane. Schematic inset illustrates the moderately developed double-arcuate pattern present. (R) Vector field of $\mathbf{u}_{1}$ throughout the entire proximal tibia of Dromaius novaehollandiae (QMO 11686), illustrating increasing obliquity and disorganization of vectors in the distal metaphysis and transition to the diaphysis (region with braces). 


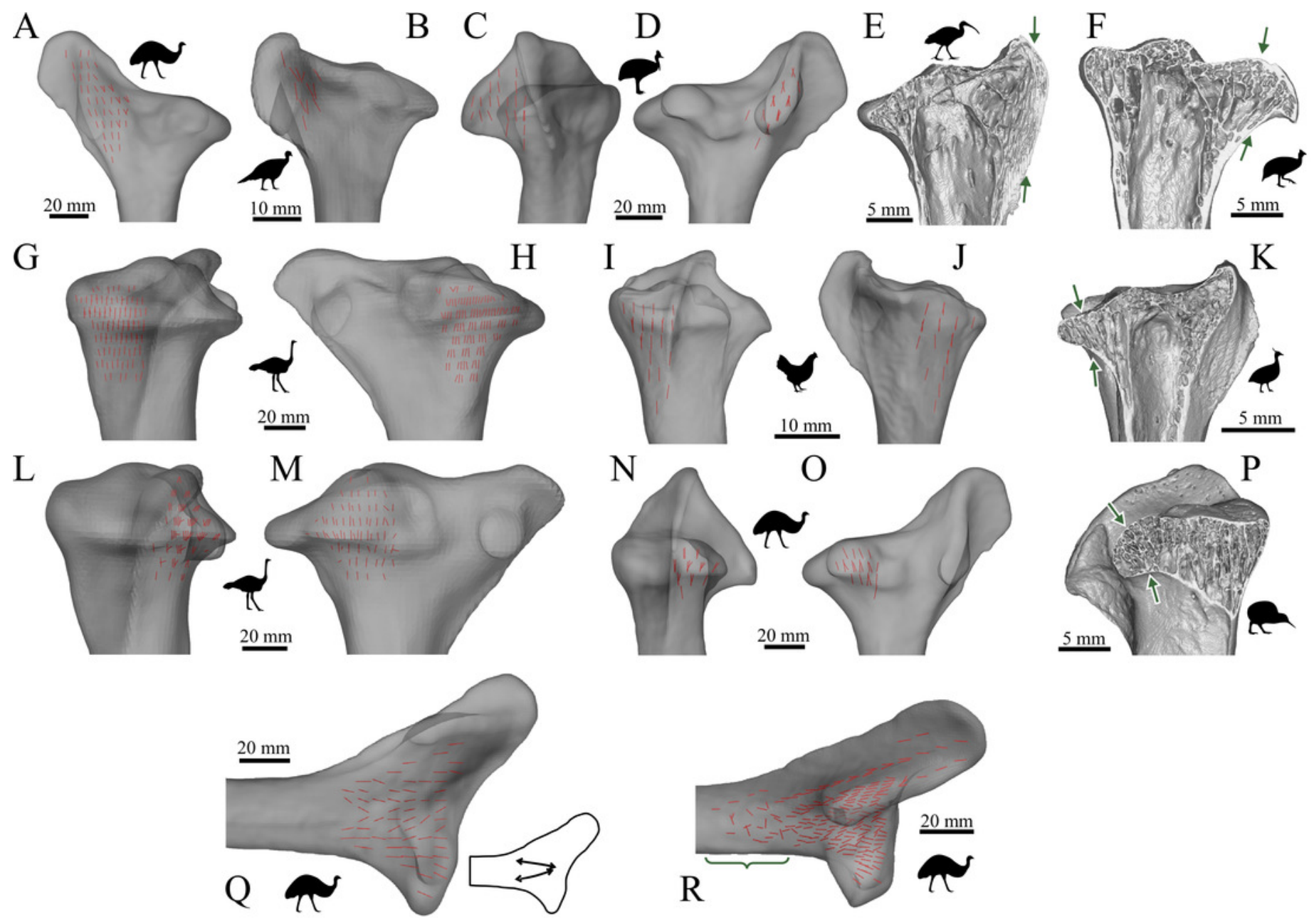


Figure 32

The main architectural features of cancellous bone in the proximal tibia of extant sprawling reptiles, as exemplified by a saltwater crocodile, Crocodylus porosus (QM) 48127).

(A) Vector field of $\mathbf{u}_{1}$ in anterior view. (B) Vector field of $\mathbf{u}_{1}$ in medial view. 


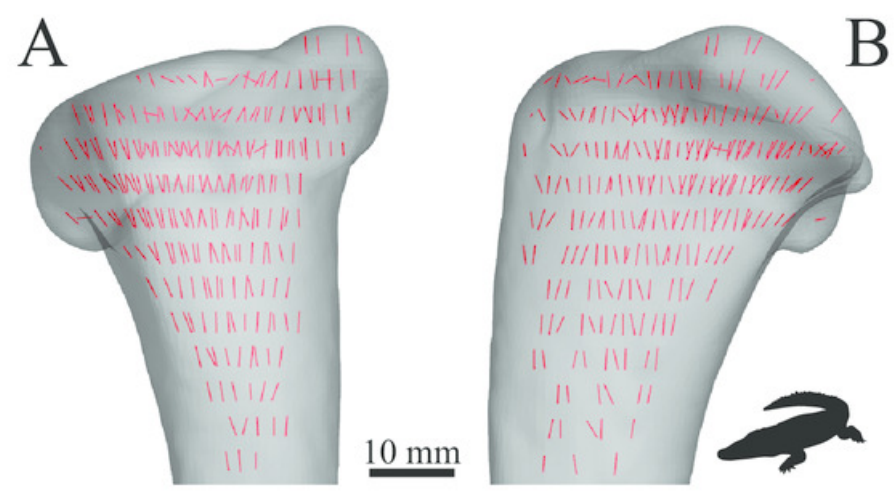




\section{Figure 33}

The main architectural features of cancellous bone in the proximal tibia of both Allosaurus and the tyrannosaurids.

These are illustrated here with a 3-D geometric model of the observed architecture, mapped to the tibia of Daspletosaurus torosus (TMP 2001.036.0001). (A) The dominant orientation of cancellous bone in the medial condyle, in medial view. (B) The dominant orientation of cancellous bone in the medial and lateral condyles, in posterior view. (C) The dominant orientation of cancellous bone in the lateral condyle, in lateral view. (D-J) Seven progressive rotations of the bone, in $30^{\circ}$ increments, from proximally oblique medial to lateral views. For explanation of the features and colour coding, refer to the main text.

A

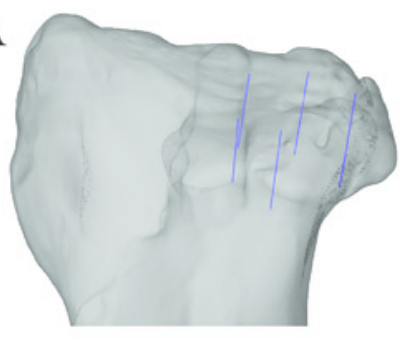

B

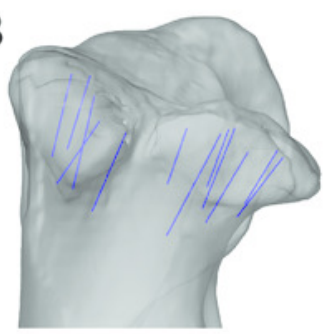

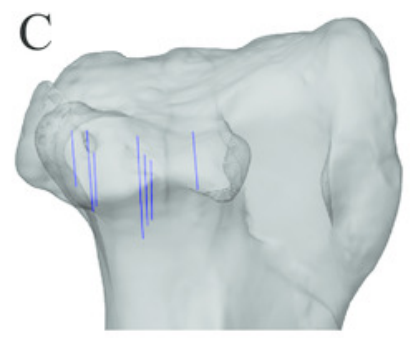

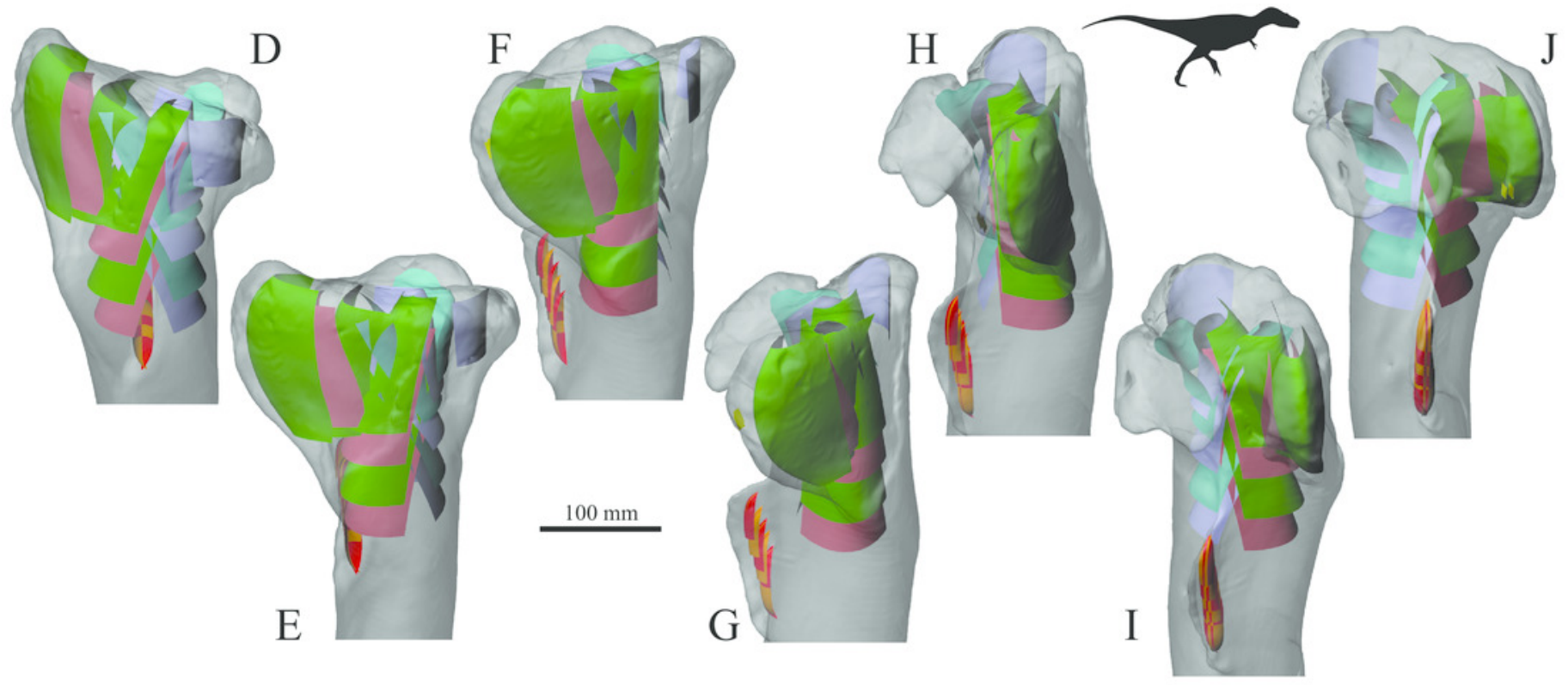




\section{Figure 34}

The main architectural features of cancellous bone in the proximal tibia of Troodontidae sp. and Saurornitholestes langstoni .

(A, B) Vector field of $\mathbf{u}_{1}$ under the medial condyle of Troodontidae sp. (MOR 553s-

7.11.91.41) in posterior (A) and medial (B) views. (C, D) Vector field of $\mathbf{u}_{1}$ under the lateral condyle of Troodontidae sp. (MOR 748) in posterior (C) and lateral (D) views. (E, F) Vector field of $\mathbf{u}_{1}$ in the cnemial crest of Troodontidae sp. (MOR 748) in lateral (E) and anterior (F) views. (G) Vector field of $\mathbf{u}_{1}$ in the cnemial crest of Saurornitholestes (MOR 660) in lateral view. $(H)$ Vector field of $\mathbf{u}_{1}$ in a 3-D slice, parallel to the sagittal plane, through the central metaphysis of Troodontidae sp. (MOR 553s-7.28.91.239), shown in medial view. Schematic inset illustrates the moderately developed double-arcuate pattern present. 
A

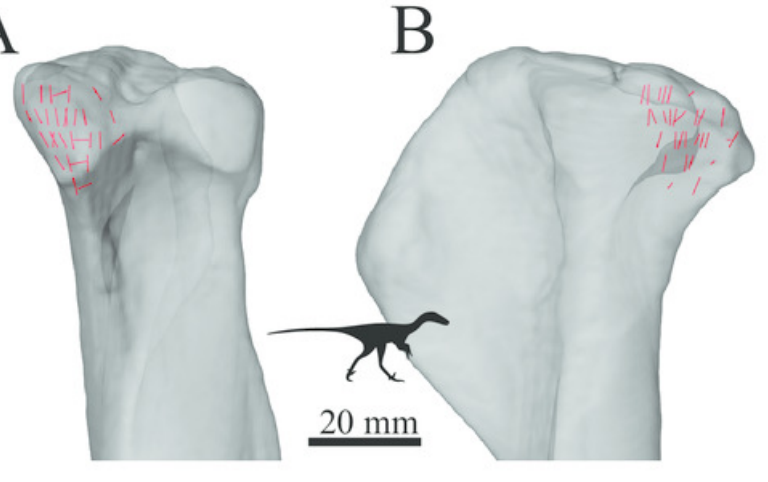

E

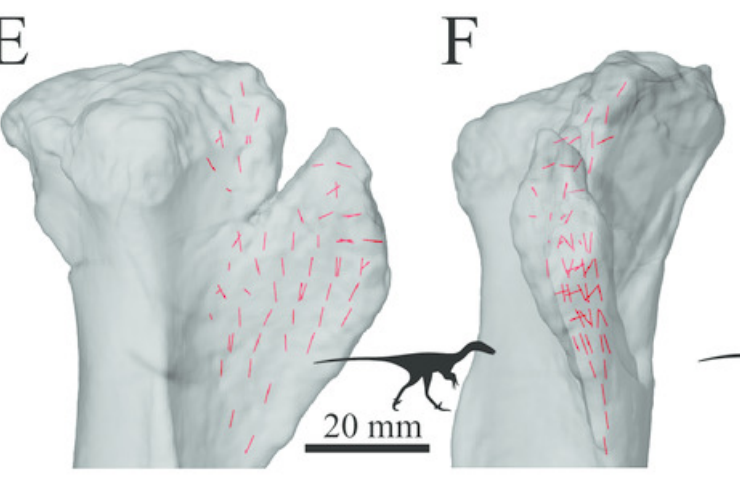

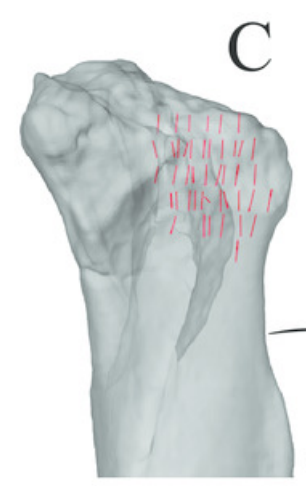

C
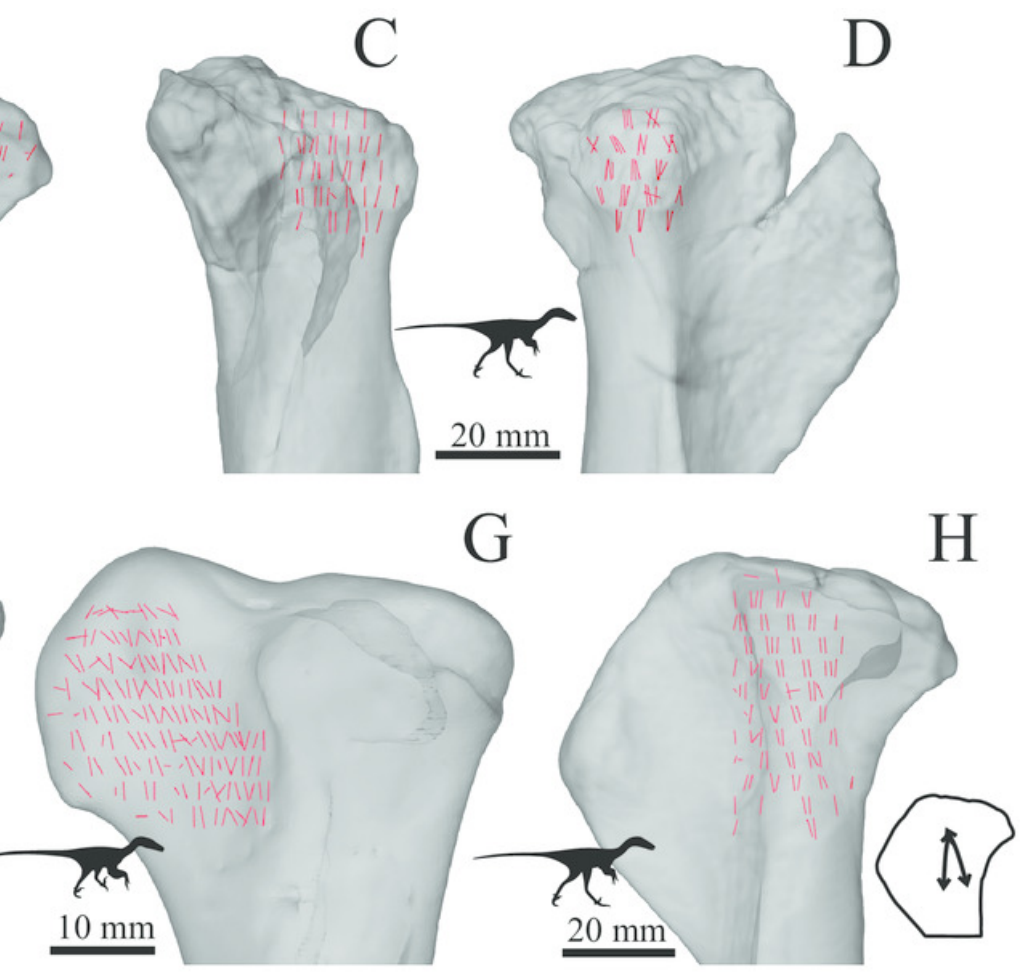


\section{Figure 35}

The main architectural features of cancellous bone in the human distal tibia.

(A) Vector field of $\mathbf{u}_{1}$ in a 3-D slice, parallel to the coronal plane, through the middle of the bone, shown in anterior view. (B) Vector field of $\mathbf{u}_{1}$ in a 3-D slice, parallel to the sagittal plane, through the middle of the bone, shown in lateral view. (C) Vector field of $\mathbf{u}_{2}$ in a 3-D slice, parallel to the axial plane, through the distal end of the bone, shown in proximal view (anterior is toward top of page). Inset shows location of slice. 

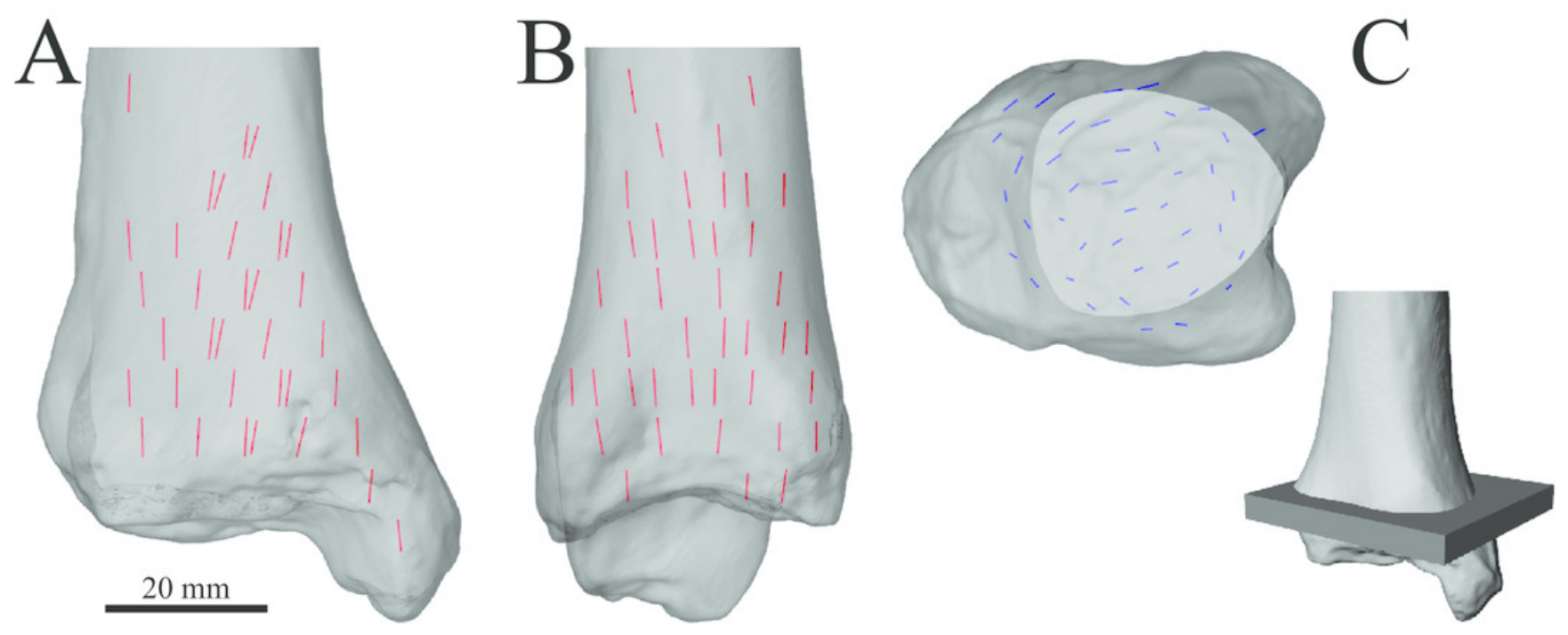


\section{Figure 36}

The main architectural features of cancellous bone in the distal tibiotarsus of birds.

(A-D) Vector field of $\mathbf{u}_{1}(A, C)$ and $\mathbf{u}_{2}(B, D)$ in the distal tibiotarsus of an emu, Dromaius novaehollandiae (QMO 16140), in oblique anterolateral (A, B) and oblique anteromedial (C, D) views. (E) Vector field of $\mathbf{u}_{1}$ (red) and $\mathbf{u}_{2}$ (blue) in the condyles of Dromaius novaehollandiae (QMO 16140) in proximal view (anterior is toward top of page). Note how both $\mathbf{u}_{1}$ and $\mathbf{u}_{2}$ are strongly aligned parallel to the sagittal plane. This particular specimen exemplifies a very stereotypical pattern that is observed in all large birds; the general pattern illustrated here was also observed in smaller species for which only limited fabric analysis was possible. (F) Isosurface rendering of cancellous bone in the distal tibiotarsus of a southern cassowary, Casuarius casuarius (QMO 30105), shown in oblique anteromedial view, with multiple cuts through the bone to illustrate 3-D architecture. (G) Isosurface rendering of cancellous bone in the distal tibiotarsus of a bustard, Ardeotis australis (MVB 20408), shown in oblique anterolateral view, with multiple cuts through the bone to illustrate 3-D architecture. $(H)$ Isosurface rendering of cancellous bone in the distal tibiotarsus of a painted quail, Coturnix chinensis (PJB coll.), sectioned in the axial plane through the middle of the condyles and shown in proximal view (anterior is toward top of page). In F and G, cut surfaces are coloured red to better show the nature of the cancellous bone architecture, in particular, the plate-like nature of many of the trabeculae, largely aligned parallel to the sagittal plane. 

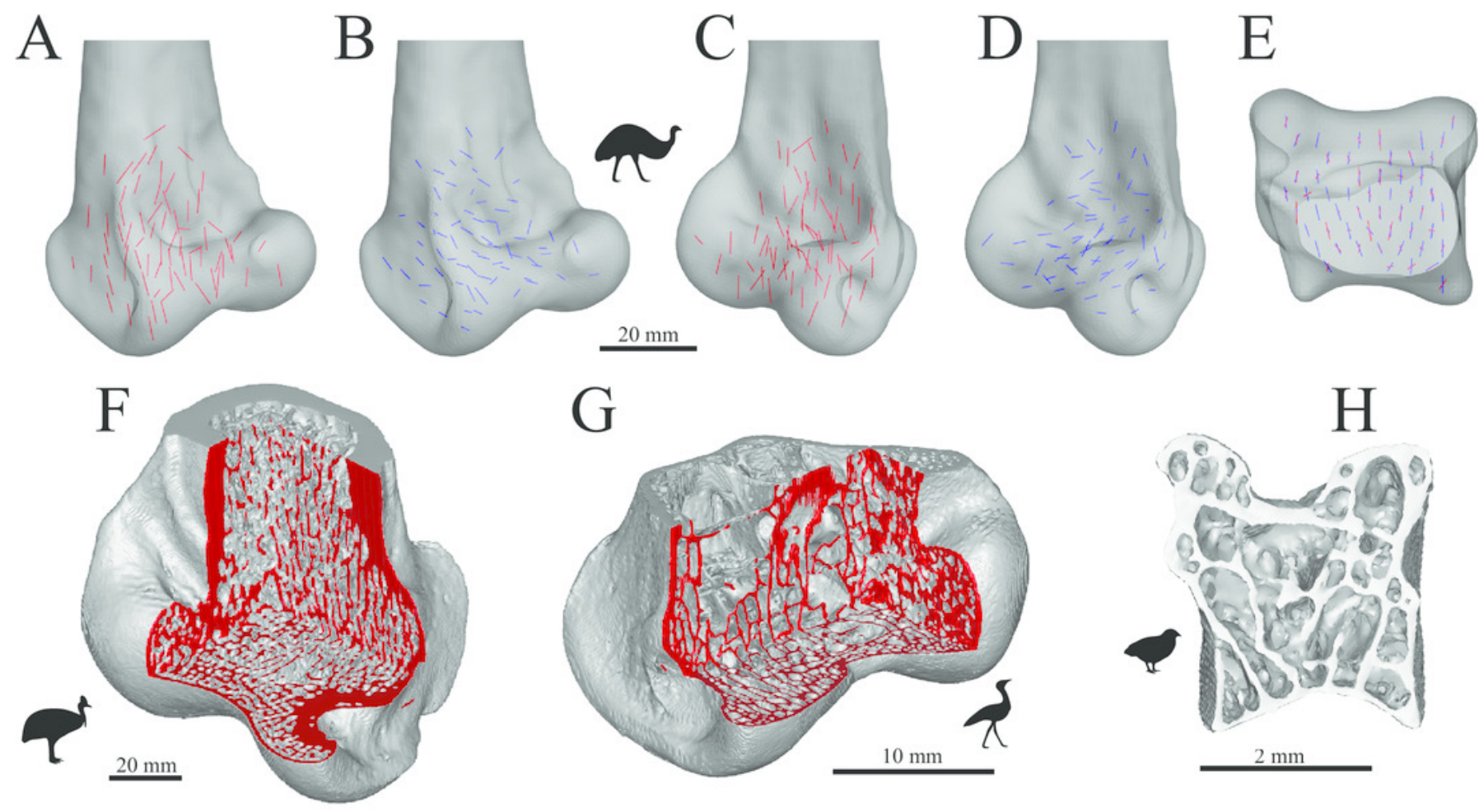

G
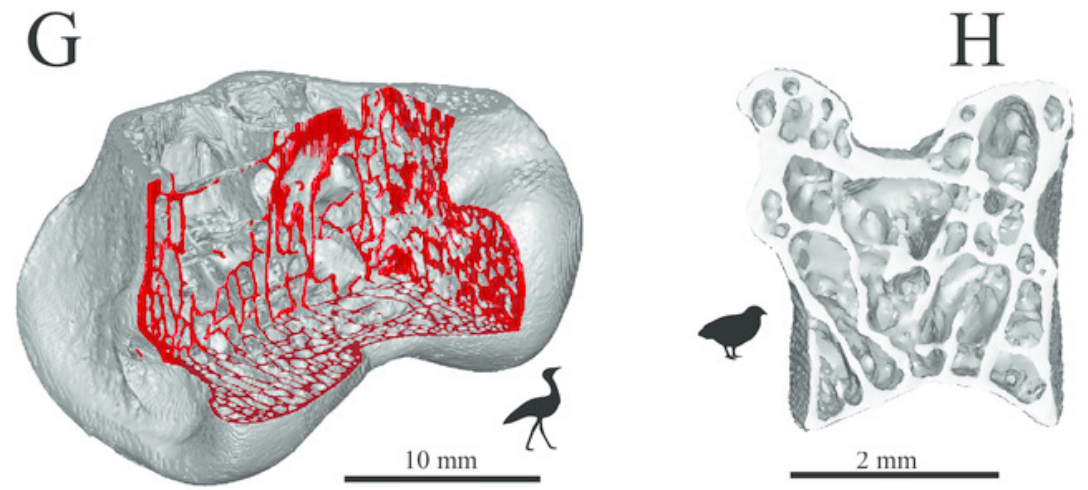


\section{Figure 37}

The main architectural features of cancellous bone in the distal tibia of extant sprawling reptiles.

(A, B) Vector field of $\mathbf{u}_{1}$ in a Komodo dragon, Varanus komodoensis (AM R.106933) in anterior (A) and medial (B) views. (C) Vector field of $\mathbf{u}_{1}$ in a saltwater crocodile, Crocodylus porosus (QMJ 48127) in anteromedial view. (D) Vector field of $\mathbf{u}_{2}$ in a 3-D slice through the distal end of the tibia of Crocodylus porosus (QMJ 48127), shown in proximal view (anterior is toward top of page). Inset shows location of slice. 


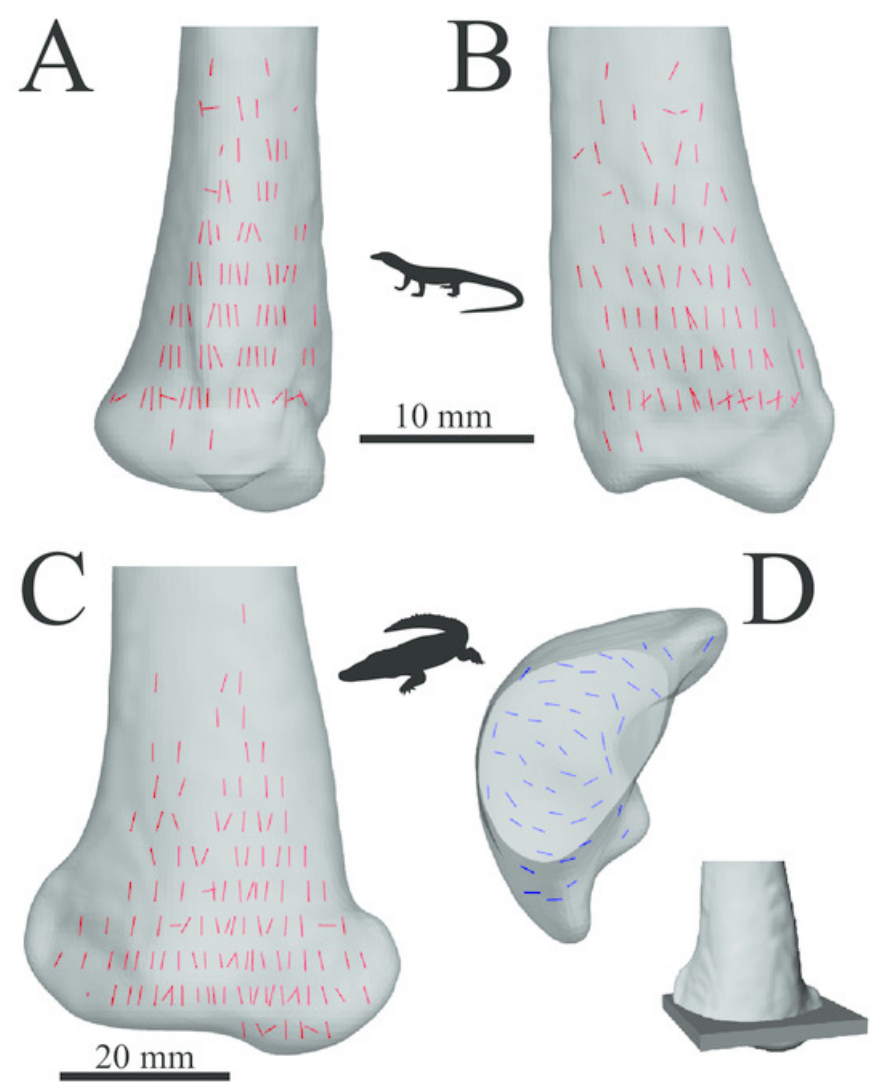




\section{Figure 38}

The main architectural features of cancellous bone in the distal tibiotarsus of basal theropods.

These are illustrated here with a 3-D geometric model of the observed architecture, mapped to the tibiotarsus of Daspletosaurus torosus (TMP 2001.036.0001; note that calcaneum was digitally sculpted based on other tyrannosaurid calcanei). (A-G) Seven progressive rotations of the tibia, in $30^{\circ}$ increments, from proximally oblique medial to lateral views. Schematic inset in D illustrates generic cross-sectional pattern at the level indicated. (H-J) Three views of the astragalus and calcaneum, corresponding to those in $C-E$, respectively. The various colours are used to help visualize the various tracts of cancellous bone more clearly.

A

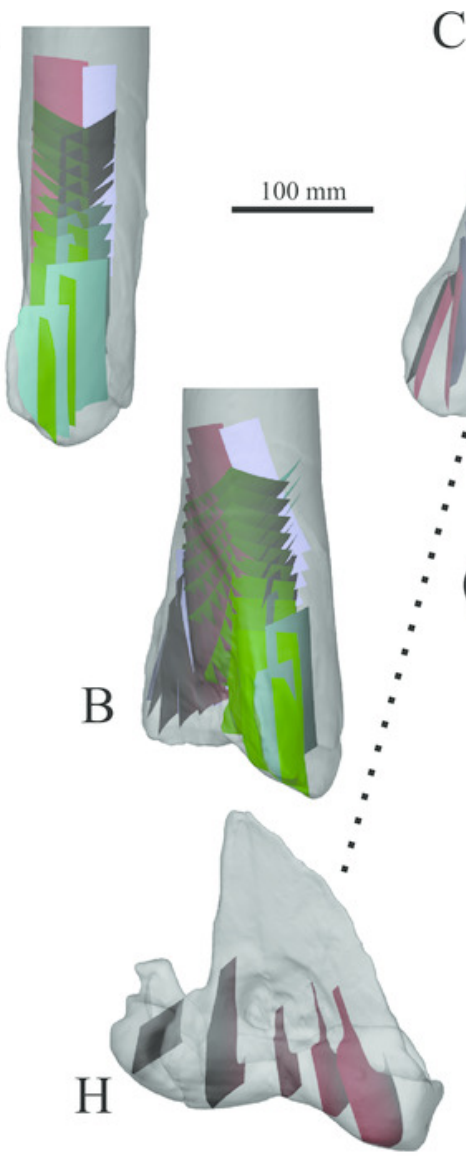

$\mathrm{C}$
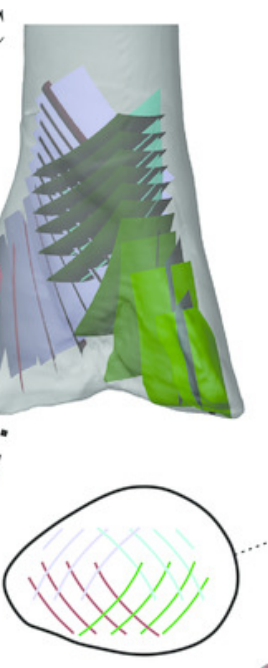
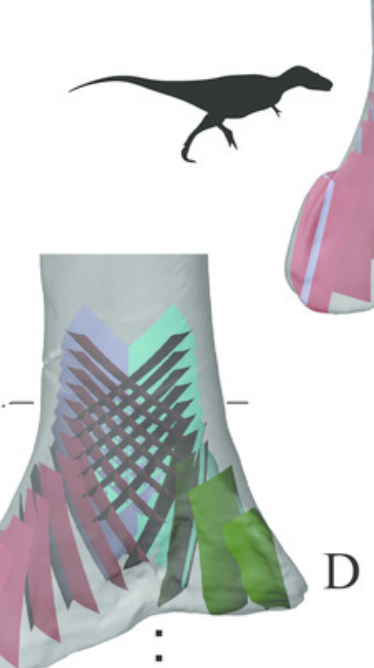

D

E
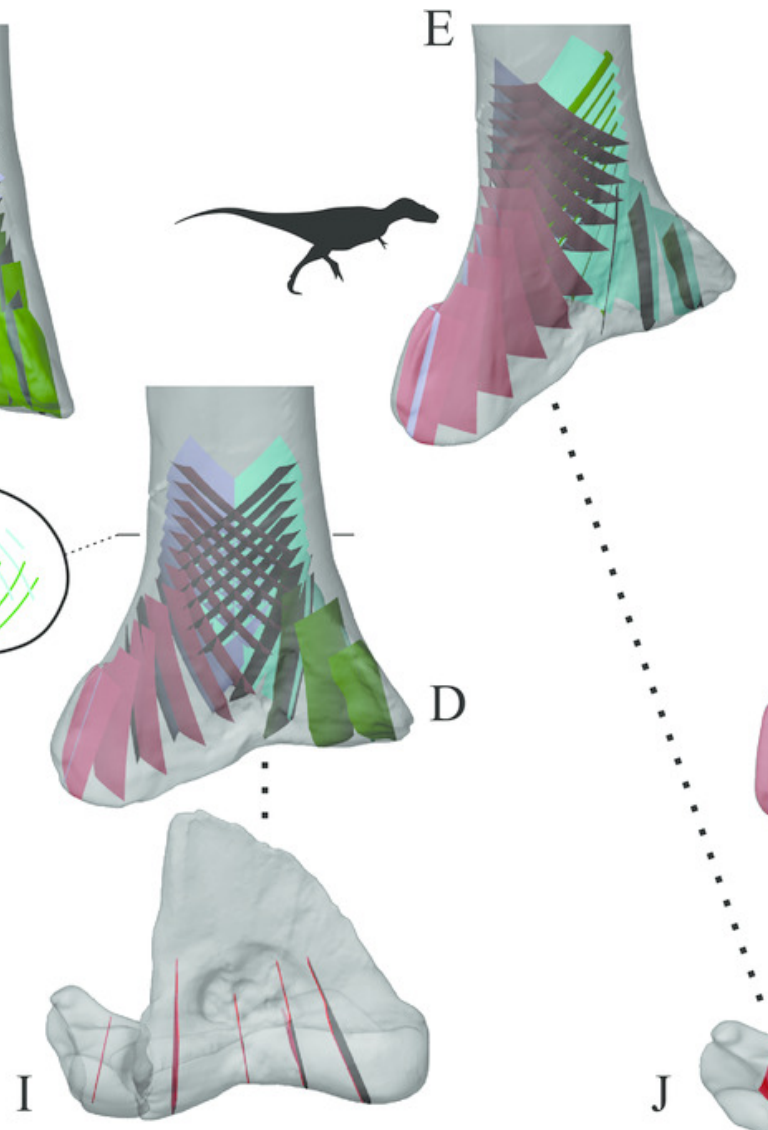

J

G
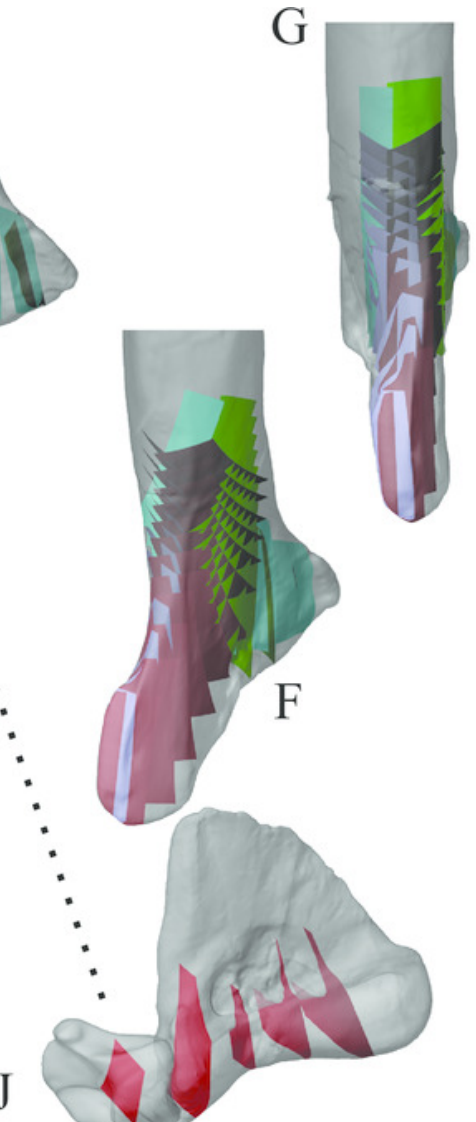

4
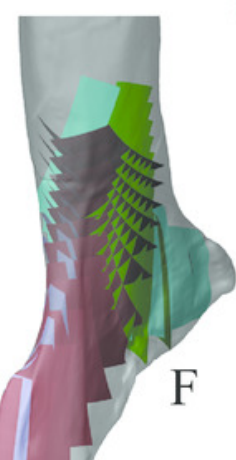

s




\section{Figure 39}

The main architectural features of cancellous bone in the distal tibiotarsus of Troodontidae sp. and Saurornitholestes langstoni .

(A, B) Vector field of $\mathbf{u}_{1}$ (red) and $\mathbf{u}_{2}$ (blue) in the distal tibiotarsus of Troodontidae sp. (MOR 748) in anterior (A) and proximal (B) views; in B, anterior is toward top of page. Note how both $\mathbf{u}_{1}$ and $\mathbf{u}_{2}$ are generally aligned parallel to the sagittal plane. (C) Isosurface rendering of cancellous bone in the medial distal tibia of Saurornitholestes (MOR 660), shown in oblique anteromedial view, with multiple cuts through the bone (cut surfaces are coloured red) to illustrate 3-D architecture. 


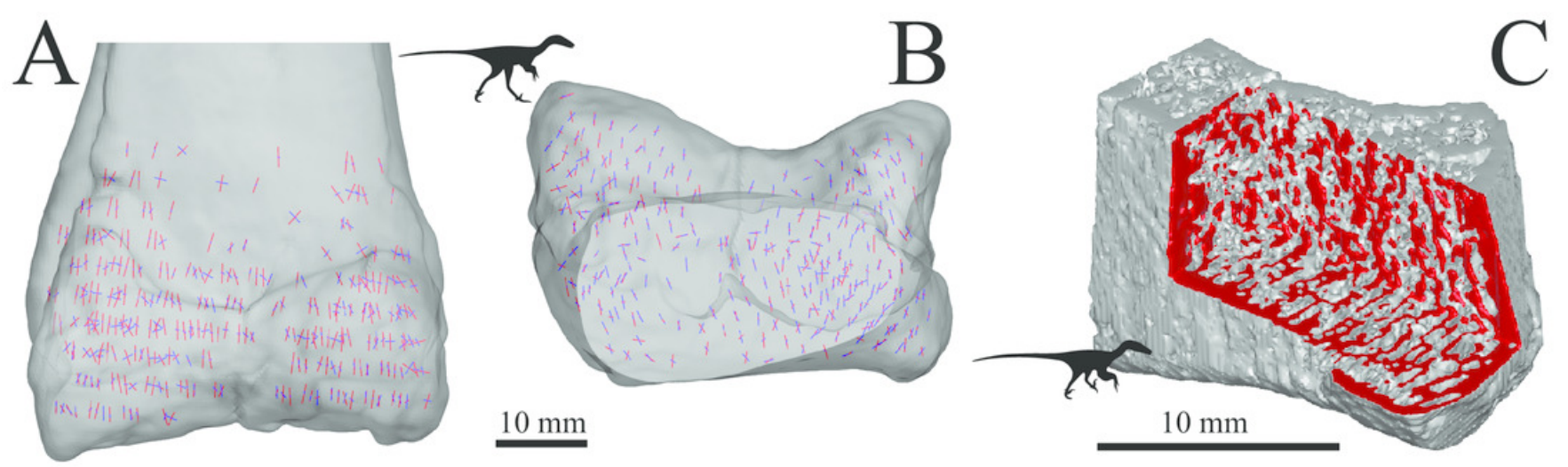




\section{Figure 40}

The main architectural features of cancellous bone in the fibula.

(A, B) Vector field of $\mathbf{u}_{1}$ in the human fibula, in anterior (A) and lateral (B) views. (C, D) Vector field of $\mathbf{u}_{1}$ in a freshwater crocodile, Crocodylus johnstoni (QMJ 47916), in anterior (C) and lateral (D) views. (E, F) Vector field of $\mathbf{u}_{1}$ in an Argus monitor, Varanus panoptes (QMJ 91981), in anterior (E) and lateral (F) views. (G-I) Vector field of $\mathbf{u}_{1}$ in the fibular head of an emu, Dromaius novaehollandiae (QMO 11686, G), greater rhea, Rhea americana (QMO 23517, H), and chicken, Gallus gallus (PJB coll., I), in lateral view. (J, K) Isosurface rendering of cancellous bone in the proximal fibula of a malleefowl, Leipoa ocellata (MVB 20194, J), and painted quail, Coturnix chinensis (PJB coll., K), sectioned in the plane of the head and shown in lateral view. (L, M) The dominant architectural direction of cancellous bone in the fibula of Allosaurus and tyrannosaurids, shown in anterior (L) and lateral (M) views. This is illustrated here with a 3-D geometric model of the observed architecture, mapped to the fibula of Daspletosaurus torosus (TMP 2001.036.0001). (N) Vector field of $\mathbf{u}$ ${ }_{1}$ in the proximal fibula of Troodontidae sp. (MOR 553s-8.17.92.265), in lateral view. 

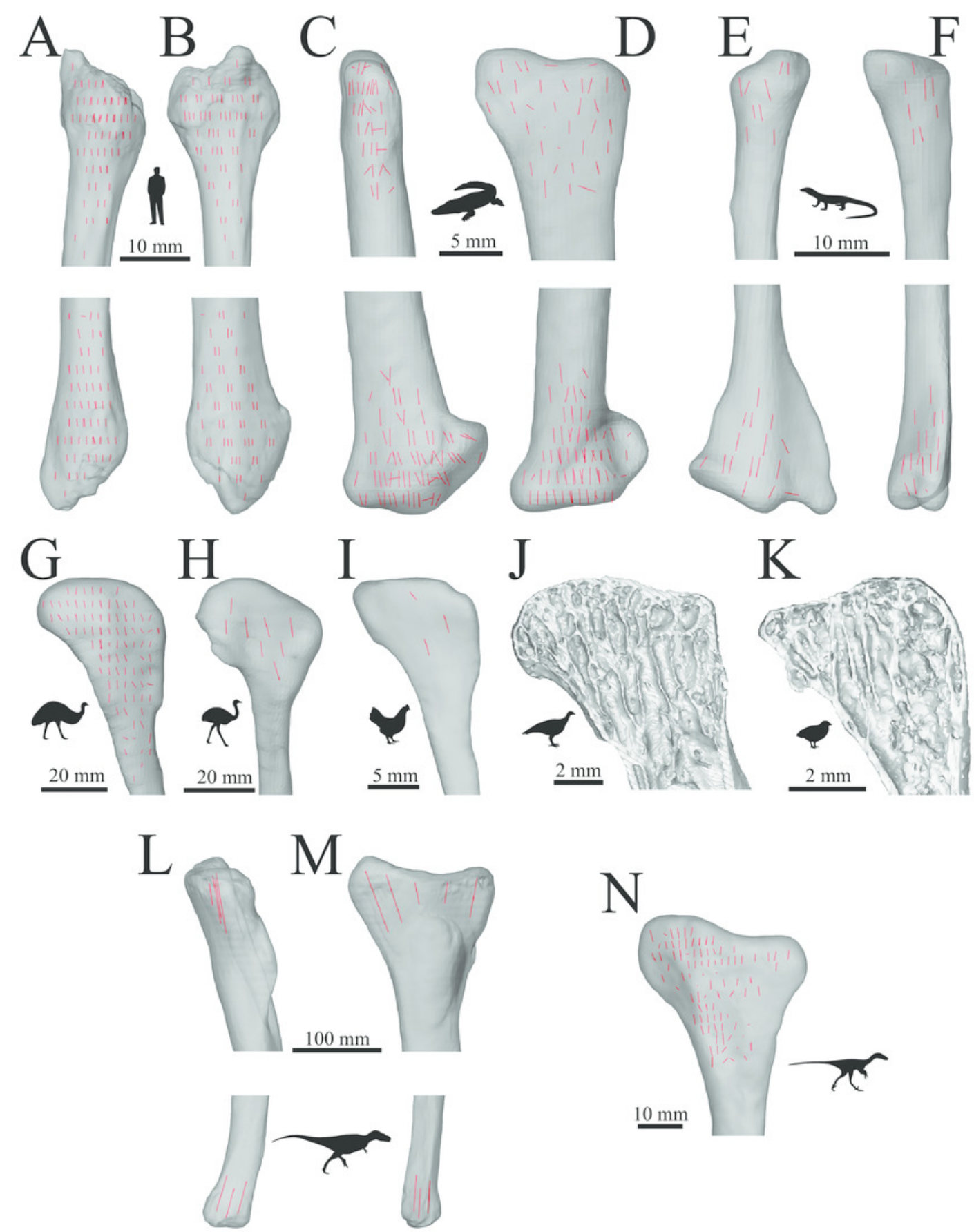


\section{Figure 41}

Oblique trabeculae in the diaphyses of birds and reptiles.

These are illustrated here with a number of examples demonstrating the variety of forms the individual trabeculae can assume. (A) Femoral mid-shaft of an emu, Dromaius novaehollandiae (QMO 16140). (B) Proximal femoral shaft of a turkey, Meleagris gallopavo (PJB coll.). (C) Distal femoral shaft of a malleefowl, Leipoa ocellata (MVB 20194). (D) Proximal femoral shaft of Dromaius novaehollandiae (QMO 11686). (E) Proximal femoral shaft of an Australian brush turkey, Alectura lathami (PJB coll.). (F) Proximal femoral shaft of an elegant-crested tinamou, Eudromia elegans (UMZC 404.e). (G) Femoral mid-shaft of an Argus monitor, Varanus panoptes (QMJ 91981). (H) Distal femoral shaft of a little tinamou, Crypturellus soui (MVB 23647). (I) Tibial mid-shaft of an ostrich, Struthio camelus (MV R.2385). In all figures, proximal is towards the top of the page. 

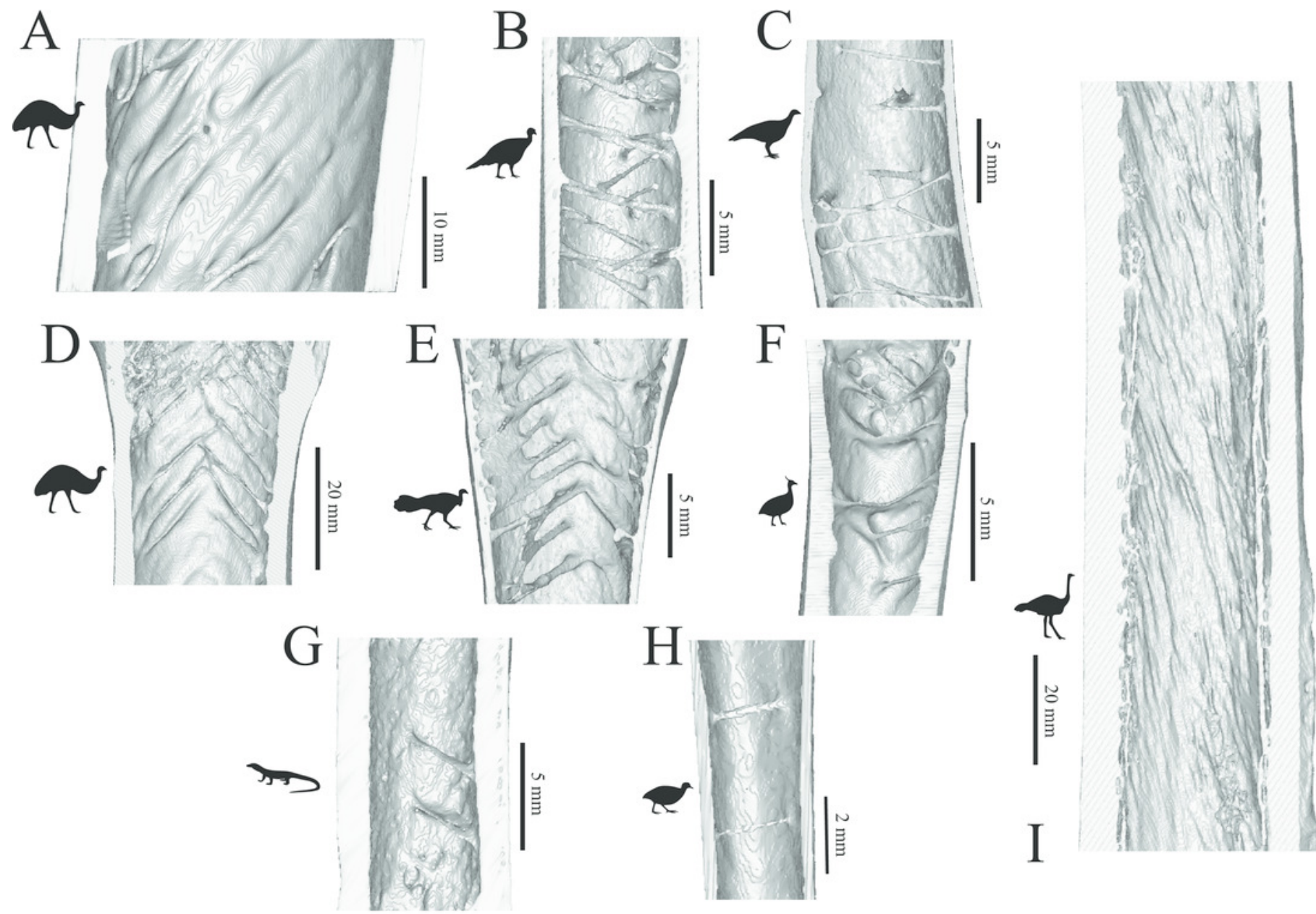


\section{Figure 42 (on next page)}

Size-dependent variation in diaphyseal cancellous bone architecture in the femora and tibiae of birds.

These plots compare the results of the categorical scoring of each bone against its length. (A, B) Feature 1 (extent of cancellous bone) in the femur (A) and tibia (B); a higher score indicates greater extent. (C, D) Feature 2 (average orientation of trabeculae) in the femur (C) and tibia (D); a higher score indicates that trabeculae are more perpendicular to the bone's long-axis. (E, F) Feature 3 (degree of association of trabeculae) in the femur (E) and tibia (F); a higher score indicates that trabeculae tend to be more closely associated with other similar trabeculae. Major axis regression lines are also plotted when correlations were statistically significant. $N$ signifies number of species represented, and $n$ signifies number of individuals. Other statistical metrics for each comparison are reported in Table 2. 
A

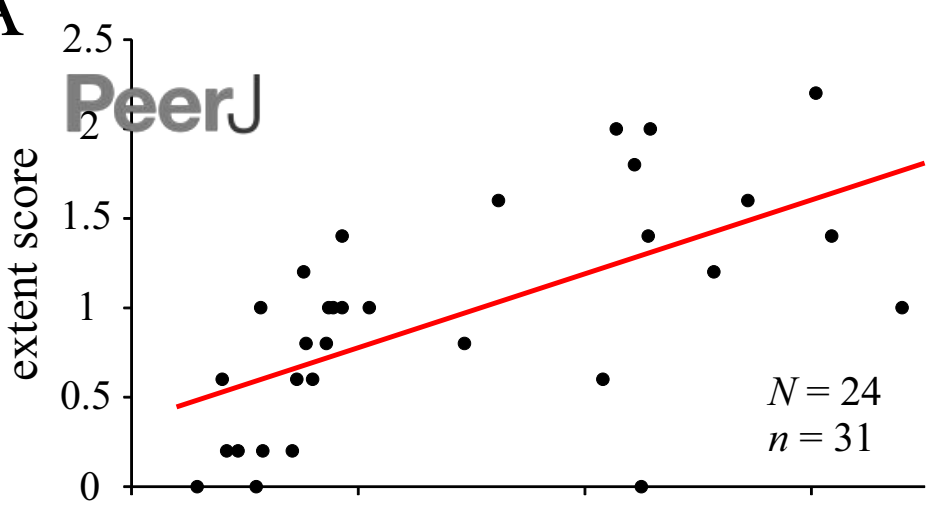

C

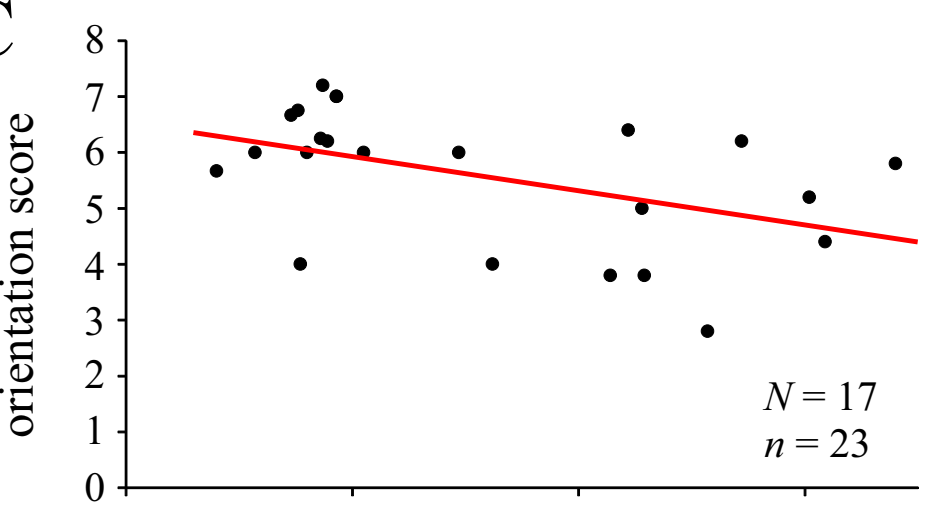

E

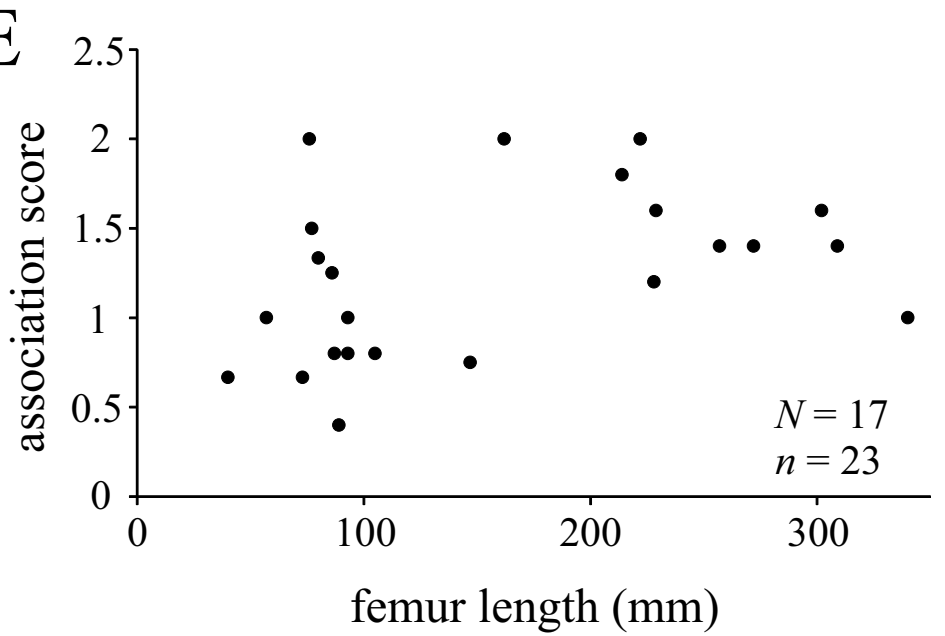

B

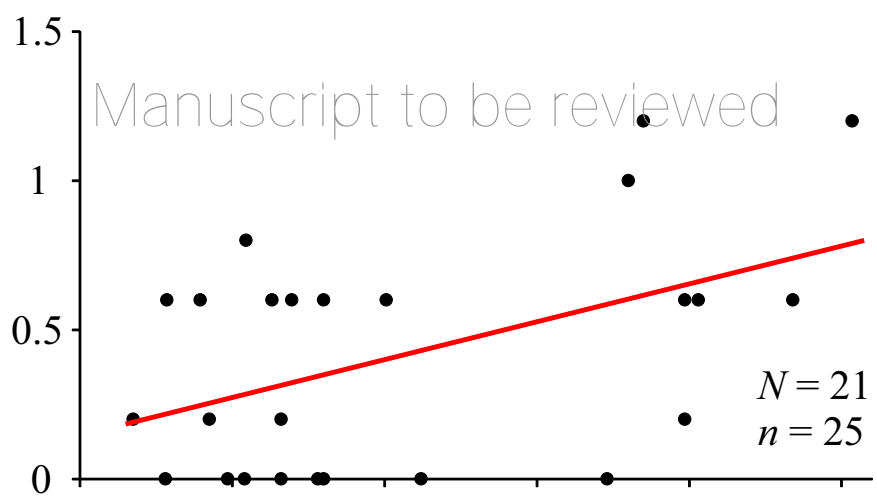

D

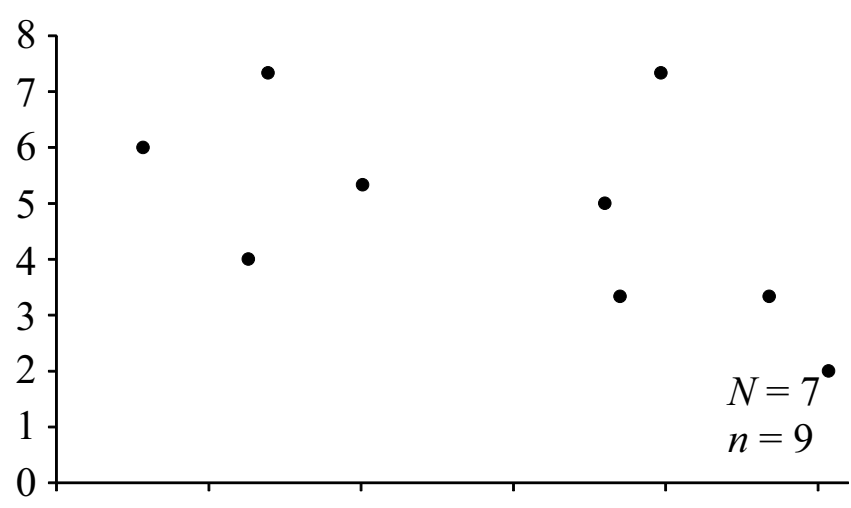

F

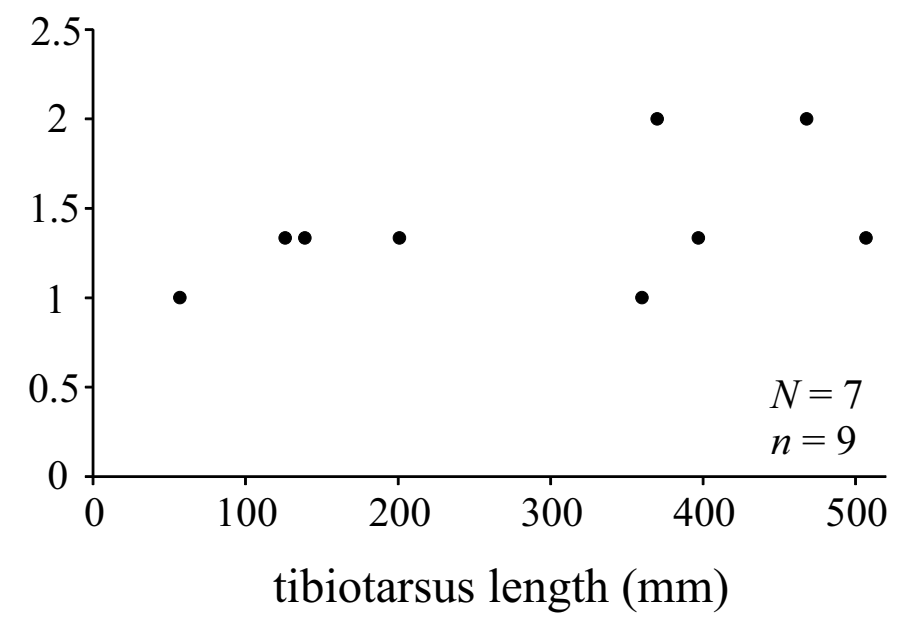




\section{Figure 43}

Gently oblique trabeculae in the tibial diaphysis of a human.

Inset shows location of section relative to the whole bone. Although the trabeculae (or endosteal 'wrinkles') are less obliquely oriented compared to the birds or reptiles, they are nonetheless consistently oriented in an oblique fashion. 


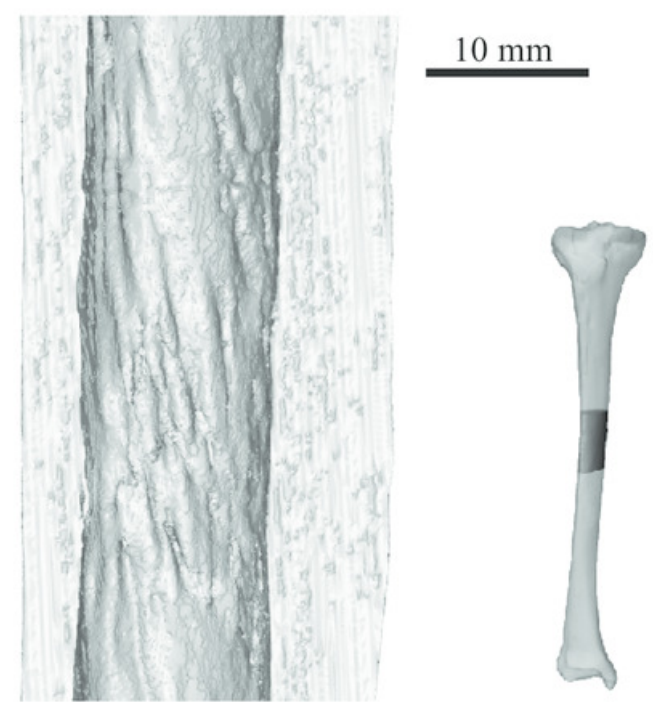




\section{Figure 44(on next page)}

Summary of the main cancellous bone architectural patterns observed in the theropods studied, placed in context of their phylogenetic relationships.

Architectural patterns were discretized into six multistate characters, and optimized to phylogeny using equal-weighted, parsimony-based ancestral state reconstruction in Mesquite 3.40 (https://www.mesquiteproject.org/). Ancestral states are shown for each node, and character state changes along branches leading to terminal taxa are given in boxes.

Characters (as letters) and states (as numbers) were defined as follows: $A 0, u_{1}$ in the femoral head has little anterior inclination; $A 1, u_{1}$ in femoral head has significant anterior inclination; $\mathrm{A}_{2}, \mathrm{u}_{1}$ in femoral head is oriented anteroposteriorly; $\mathrm{BO}, \mathrm{u}_{1}$ in femoral condyles has small posterior inclination, with minimal anteroposterior sweeping; $B 1, \mathrm{u}_{1}$ in femoral condyles has markedly increased posterior inclination; B2, $\mathrm{u}_{1}$ in femoral condyles often has strong posterior inclination, with substantial anteroposterior sweeping; $\mathrm{C} 0$, coronal-plane double arcuate pattern present in proximal femur; $\mathrm{Cl}$, proximal femur lacks coronal-plane double arcuate pattern; D0, sagittal-plane double arcuate pattern present in proximal tibia between condyles and cnemial crest; D1, sagittal-plane double-arcuate pattern in proximal tibia not as widely observed (present only in larger bones); E0, double set of double-arcuate patterns in distal tibia, juxtaposed with highly anisotropic, sagittally aligned fabric in proximal tarsals; E1, highly anisotropic, sagittally aligned fabric throughout both distal tibia and proximal tarsals; F0, fabric near diaphyses is subparallel to bone's long-axis; F1, increased obliquity of fabric nearer the femoral diaphysis; F2, fabric strongly oblique near femoral diaphysis, and oblique trabeculae can extensively invade femoral diaphysis. 


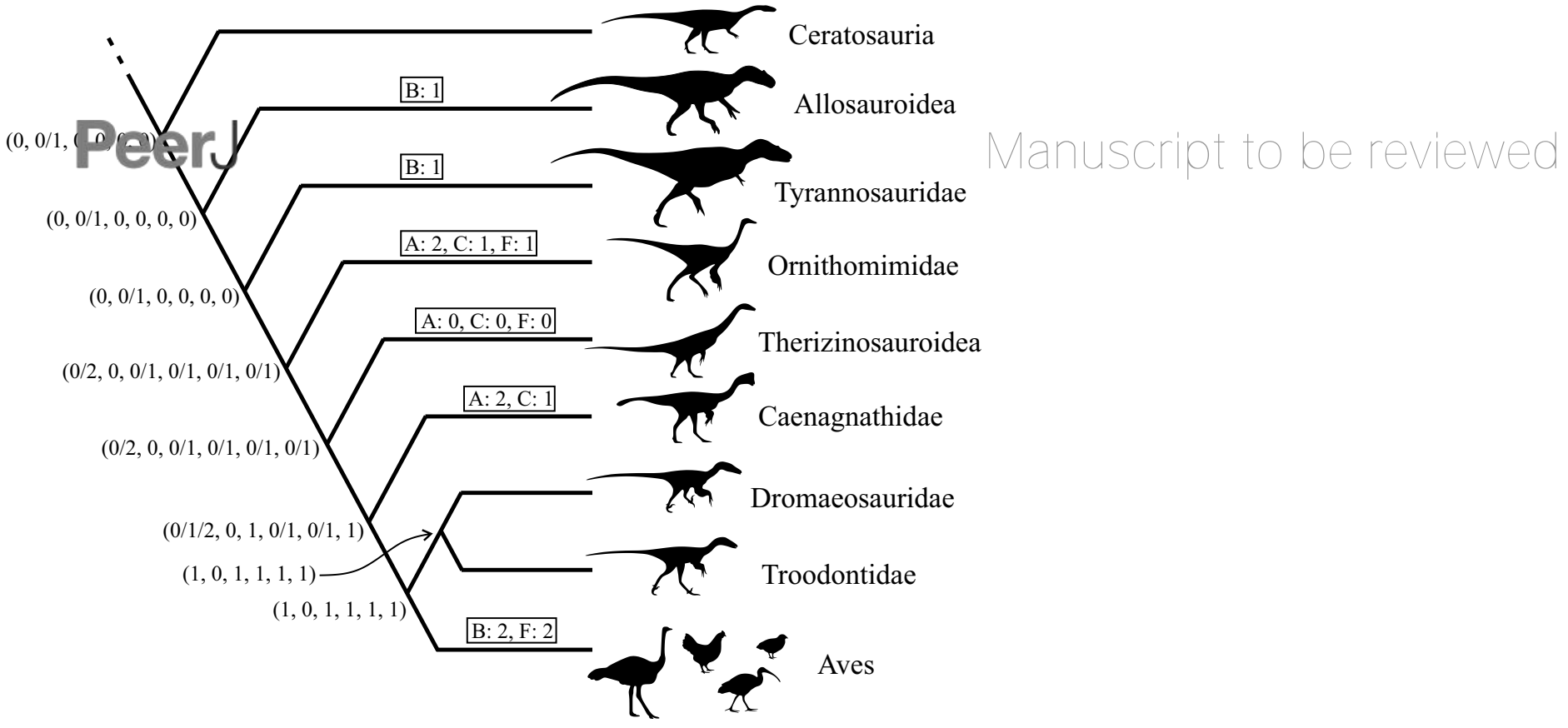


Figure 45 (on next page)

Schematic demonstrating the effect of differences in the degree of hip and knee flexion on the joint forces experienced by the femur.

This is illustrated with right lateral views of a human (A) and a typical bird (B) in approximate mid-stance postures. In the more flexed posture of birds, the hip joint force is more anteriorly oriented relative to the long-axis of the femur (dotted line) compared to humans. Further, the knee joint force is more posteriorly oriented relative to the long-axis of the femur compared to humans. 
A

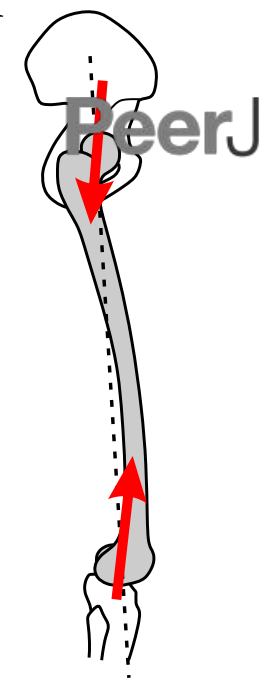

B

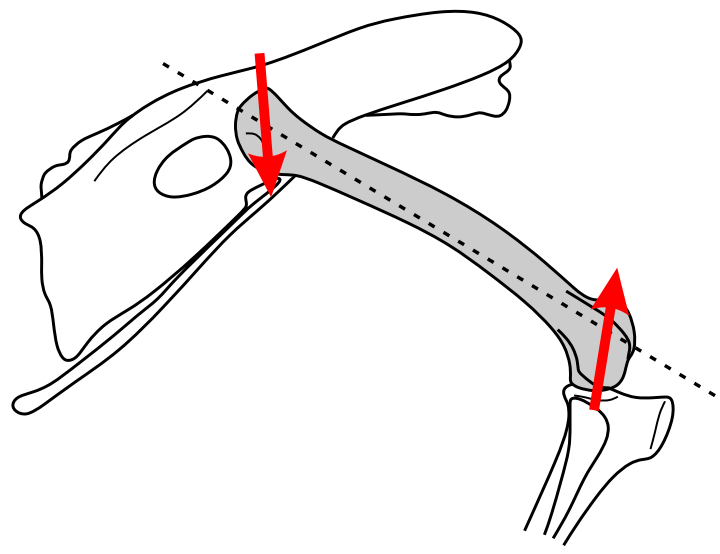

